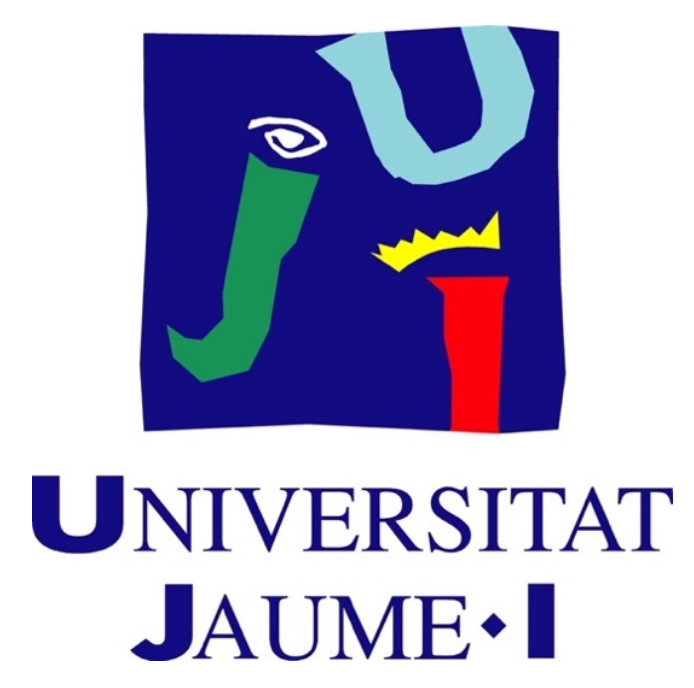

Doctoral Programme in Industrial Technologies and Materials Escuela de Doctorado de la Universitat Jaume I

\title{
Coatings of Controlled Thickness for Efficient Solid-Phase Presentation of Growth Factors
}

Doctoral Thesis presented by Andrés Alba Pérez at the Universitat Jaume I

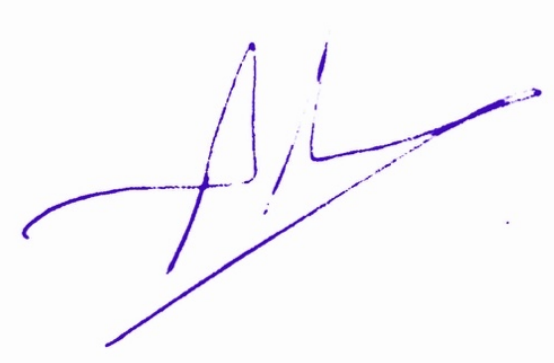

Phd candidate

Andrés Alba Pérez

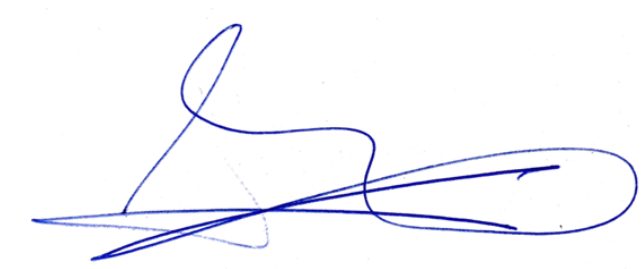

Director

Prof. Manuel Salmerón Sánchez

Castelló de la Plana, October 2020 


\section{Funding}

This thesis has been performed with support from the United Kingdom Engineering and Physical Sciences Research Council (EP/P001114/1) and the Sir Bobby Charlton Foundation. Also, a grant from the UK Regenerative Medicine Platform (MR/R015651/1) is acknowledged. 


\section{Acknowledgments}

First and foremost, I would like to acknowledge here the several structures in our shared society that have allowed me to present this Doctor of Philosophy Thesis and allow me to continue to fulfil my love to work on the problems that matter. From the education system that has raised me from a Primary School in Castellón, to Valencia, Lyon, Glasgow, and back to Castellón into a Doctoral Program at UJI, to the healthcare system that has taken care of me and my family, to all the other structures that have allowed me in obvious or not so evident ways to deliver this Thesis. I am passionate about science and I am passionate about science contributing to a better future for humanity. I am very thankful that society has provided for me to train and prepare for this responsibility, to improve everyone's future in a shared quest for knowledge, and I pledge to pursue this goal to the best of my ability.

Most of the work in this thesis has been directly financed by research grants from the UK Medical Research Council and from The Sir Bobby Charlton Foundation, at the time Find A Better Way Charity.

I have been able to accomplish this thesis thanks to the help and guidance of several people, some of them that I will name personally here. My Thesis Tutor Prof Antonio Pérez González and Director Prof Manuel Salmerón Sánchez. Manuel, which I have known for many years now, has been a professional and personal role model that has inspired me decidedly to complete this research work and degree. Dr Marco Cantini, Dr Cristina Gonzalez García, Dr Alex Rodrigo Navarro, Dr Virginia Llopis Hernandez, Dr Sara Trujillo, Dr Peter Childs, Dr Vineetha Jayawarna, Dr Monica

Tsimbouri, Dr Annie Zhe Cheng, and Prof Matt Dalby, all at the University of Glasgow, that helped me in many different ways over the years with their knowledge and technical proficiency and also with settling in Glasgow as a newcomer to the city. Microfab technician Mr Thomas Reilly and Dr Paul Reynolds, that helped hugely with understanding and setting up a plasma reactor and shared many hours in the lab and the office with me.

I am so grateful to my partner MariCarmen and my children Ismael and Daniela, the joy of my life, for their love and help in so many needed and subtle ways. I also want to thank here my parents María Teresa and Andrés and my brother José, for their patience and wisdom in raising me, encouraging me to follow my passions, however frequently they might change.

"Which path do you intend to take, Nell?' said the Constable, sounding very interested. 'Conformity or rebellion?'

Neither one. Both ways are simple-minded - they are only for people who cannot cope with contradiction and ambiguity."

— Neal Stephenson, The Diamond Age: Or, A Young Lady's Illustrated Primer 



\title{
Table of Contents
}

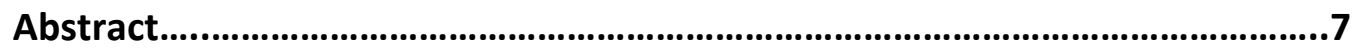

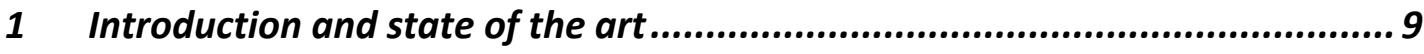 \\ $1.1 \quad$ Engineering the cell microenvironment .......................................................... 10 \\ $1.2 \quad$ Coating with polymers of biomedical interest ............................................. 11

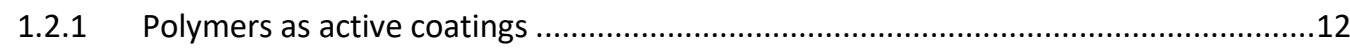 \\ 1.2.2 Polymer coatings as carriers for controlled delivery ................................................13 \\ 1.2.3 Coating with plasma polymerised materials ............................................................14
}

2 Overall aim and specific objectives................................................. 17

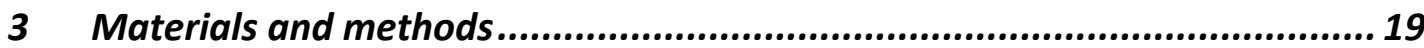

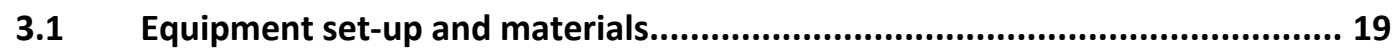

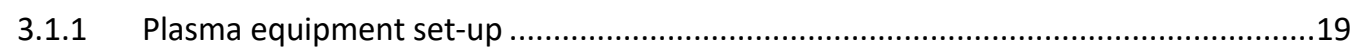

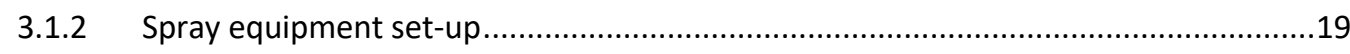

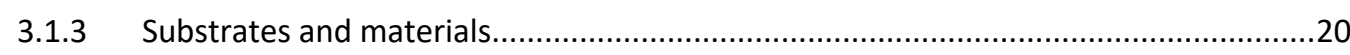

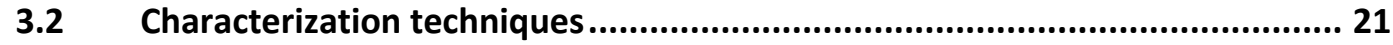

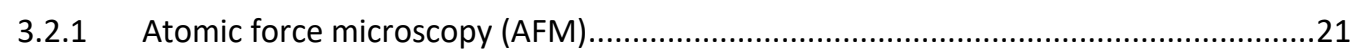

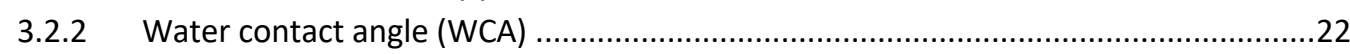

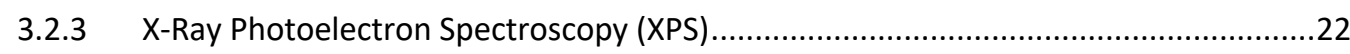

3.2.4 Protein adsorption and domain exposure assays ...................................................22

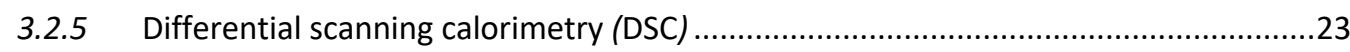

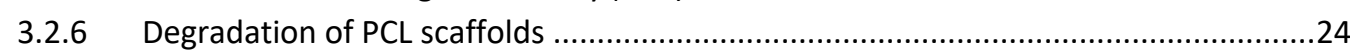

$4 \quad$ Results and discussion. Plasma coatings. .......................................... 25

4.1 Assembly of a plasma polymerization reactor .......................................... 25

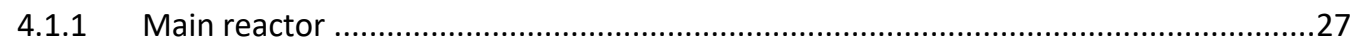

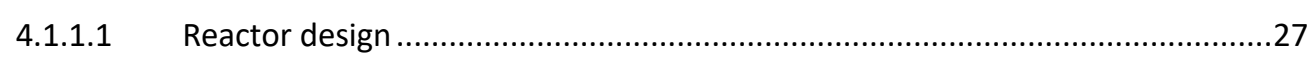

4.1.1.2 Considerations for the operation with EA monomer ......................................28

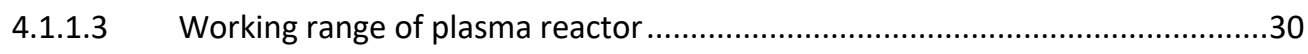

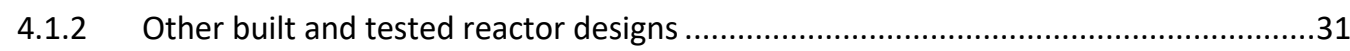

4.1.2.1 Cylindrical chamber with inner plate electrodes ...........................................32

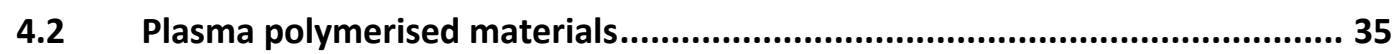

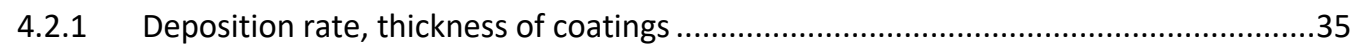

4.2.1.1 Deposition rate in different areas of the chamber ..........................................39

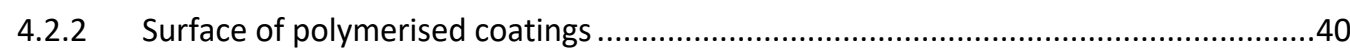

4.2.2.1 Roughness of coating on flat substrates.......................................................40

4.2.2.2 Flaky softer areas on polymerised material ...................................................47

4.2.3 Conservation of functional roughness on substrates...............................................50

4.2.3.1 Maintaining functional roughness at the sub-micron scale ...............................50

4.2.3.2 Control of coating deposition over hyper-rough surfaces.................................56

4.2.4 Water contact angle of plasma coated surfaces ........................................................58

4.2.5 Chemical analysis of plasma polymerised materials - XPS .......................................61 


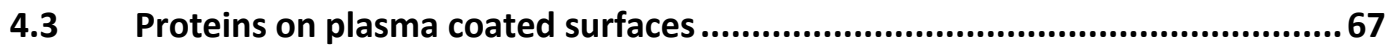

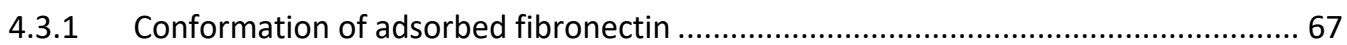

4.3.2 Conformation of adsorbed fibronectin at low concentrations .................................... 75

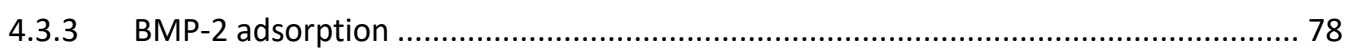

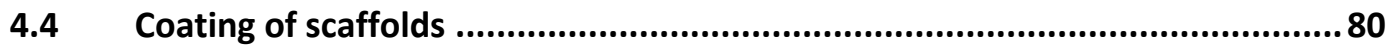

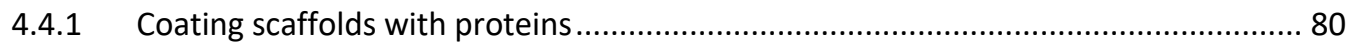

$5 \quad$ Results and discussion. Spray coatings................................................. 83

5.1 Setting up a custom-made spray installation.....................................................8.

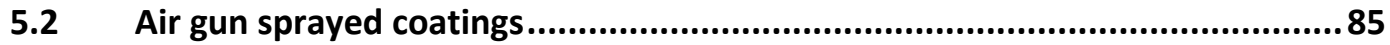

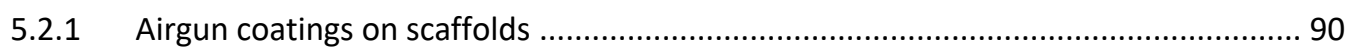

5.2.2 Fibronectin conformation on air gun coatings ......................................................... 93

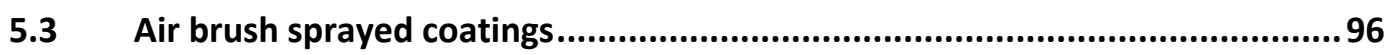

5.3.1 Degradation of the spray coatings on flat surfaces............................................... 99

5.3.2 Effect of coatings on the degradation of scaffolds............................................ 101

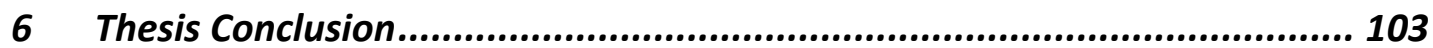

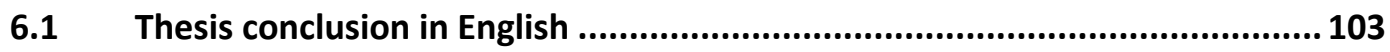

6.2 Conclusiones de la tesis en Español.............................................................. 104

6.3 Conclusions de la tesis en Valencià ............................................................... 105

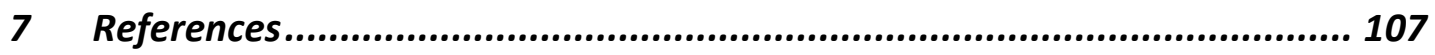

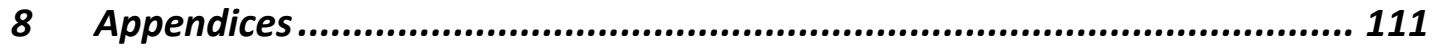

8.1 Scientific publications by the candidate ..................................................... 111

8.2 Plasma reactor operation protocol ............................................................ 112

8.3 Overview of coating protocols for scaffolds ............................................. 113

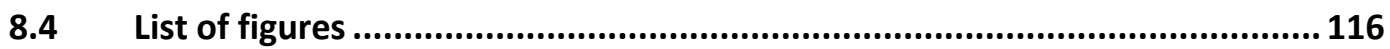

8.5 Schematic diagram of cell - ECM interactions and material-driven FN

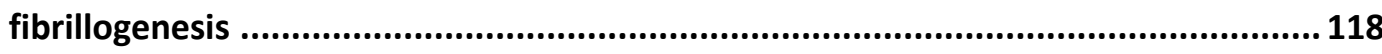





\section{Abstract}

We have developed a plasma polymerization equipment and process that uses a low power radio frequency glow discharge to coat surfaces with plasma polymerized Poly(ethyl acrylate) (PEA). Our set-up has demonstrated a fine control of the chemical integrity and the thickness of the coating, even at sub-micron scales.

We have optimised the operation parameters of this equipment and have characterised the materials produced, in particular how fibronectin behaves when coated onto them. We have systematically evaluated process conditions and their impact on the plasma polymerised PEA coatings, characterising the effect of plasma power and deposition time on thickness, wettability and chemical composition of the coatings. We also have demonstrated that substrate functional roughness can be maintained after deposition of the polymer coatings. Importantly, we show that coatings deposited at different conditions all maintain a similar or better bioactivity than spin coated PEA references. We show that fibronectin assembles into compact nanonetworks on the PEA plasma polymerised coatings, with high availability of integrin and growth factor binding regions that sequester bone morphogenetic protein-2 (BMP-2). Overall, we show that this is a potent and versatile technology that can help facilitate the use of GFs in clinical applications.

Additionally, we worked on a complementary coating technology to coat scaffolds and other structures with PEA. We built in our lab two custom-made installations to spray PEA from solutions in toluene on surfaces and scaffolds. Our first pilot installation was for a simple high-volume airgun. A second installation that allowed more precise control of the coating was for a low volume airbrush. We optimised protocols and requirements to coat surfaces and scaffolds for the two spraying installations, we developed and characterised the sprayed coatings (thickness, partial/full coating distribution, degradation in water), checked the conformation of fibronectin on sprayed surfaces with AFM, and verified the efficiency in coating 3D structures with pores and the effect on degradation rate of a biodegradable substrate (PCL). 



\section{Introduction and state of the art}

Life, at least the kind we are aware of on Earth, is characterised by interactions at different levels. For instance, distinctive and numerous chemical and physical associations between molecules occur at the cellular level, where diverse structures and signals are able to associate between them and with their environment. We have progressed greatly in understanding cellular microenvironments, the extracellular matrix (ECM) of cells, and its complex functional roles [1]. We are starting to figure out how to engineer its composition, physical properties, topology or the controlled delivery of biochemical cues. Natural and synthetic materials with biological interest can be consequently designed to behave as a synthetic ECM to influence particular aspects of the behaviour of cells [2] [3]. In many tissues, especially those involved in regenerative processes, fibronectin $(\mathrm{FN})$ is a significant ECM component that binds other matrix proteins, functional molecules such as growth factors, and cell surface receptors such as integrins [4]. FN molecules self-assemble in fibres in a natural ECM, by a cell mediated process that is of critical importance for cell-ECM interactions that subsequently occur thanks to specific FN domains being exposed in its fibrillar network state (Figure 1). A few synthetic materials have been found to induce FN fibril formation [5], and enhance exposure of integrin binding and growth factor binding domains [6] although the molecular pathways by which these materials control the biological activity of FN is not fully understood yet. Poly(ethyl acrylate) (PEA) and other polymers of the acrylate family are one of those few materials where upon adsorption of FN, physiological-like nanonetworks form that provide better availability of cell and growth factor binding regions, while adsorption on other materials leads to the formation of globular FN aggregates without full biological functionality.

Research with these materials pioneered at Prof Salmerón-Sánchez laboratory in Glasgow has shown that the fibrillar conformation of FN allows the synergistic presentation of its integrin-binding sites and bound growth factors (GF) such as bone morphogenetic protein 2 (BMP-2). These materials that drive FN fibrillogenesis are a new and formidable tool to study and control tissue regeneration, where cell mechanotransduction mechanisms and chemical signalling of growth factors are critical. Advances in this line of research have very important translational implications. The therapeutic use of growth factors is crippled by their potential adverse side effects such as tumour growth, invasion and metastasis at the unsafe supraphysiological doses they are currently used. A clinical application of BMP-2 at low concentrations that enhances mesenchymal stem cell osteogenesis and drives full regeneration of a nonhealing bone defect in vivo is possible using these materials [7], a much safer and cost-effective alternative technology.

Acrylate polymers can be processed in different ways to be used in biomedical applications. Cell and tissue support materials are developed in tissue engineering 
applications into three-dimensional scaffolds and other structures that cause desirable cellular interactions and promote the formation of new functional tissues. These structures are impossible to coat successfully with PEA with the more commonly used technologies, such as spin coating or dip coating. The coating is heterogeneous, and the solvents affect the underlaying support materials.

\subsection{Engineering the cell microenvironment}

Cells interact with their environment through cell membrane receptors like cadherins, that allow cells to attach to each other, or integrins, that attach to proteins in the ECM and connect them with the cell cytoskeleton. Molecular complexes arising from the initial interactions between integrins and ECM proteins allow cells to receive physical signals, like stiffness and force, and start complex signalling cascades as part of a variety of cellular processes, including enhance signalling from GF [8]. Integrins bind to a number of specific ligand peptides in the ECM, for instance integrin $\alpha v \beta 3$ that binds the tripeptide Arg-Gly-Asp (RGD) sequence found in fibronectin, laminin or vitronectin, or integrin $\alpha 2 \beta 1$ that binds to specific sites in collagen I and III.

Functional molecules like GF in the ECM also interact with cells through specific receptors on the cell membrane. Moreover, the signalling cascades that these receptors can trigger depend on the way the GF are available and presented in the ECM, in order for cells to properly interpret GF stimuli in different cellular settings [9]. For example, BMP-2 can be released from the ECM fibrils as a soluble ligand, but also presented to cells as a 'solid phase' ligand, bound to the ECM. BMP-2 has been widely used in the clinic soaked in a collagen sponge (Infuse and Amplify bone graft products by Medtronic Sofamor Danek, Memphis, TN) in approved anterior lumbar interbody fusion procedures but also in other off-label applications [10]. Serious side effects have been reported due to the rapid GF breakdown and clearance from target sites when delivered in this form and used at supraphysiological levels [11].

There is therefore a wider research goal to develop strategies to present GFs bound to the ECM, as an alternative to the use in the clinic of BMP-2 and other GFs at high doses with harmful consequences. In this work we are in particular interested in the use of PEA as a synthetic material that influence the interaction between integrins and GF receptors in the cell membrane, through direct physical co-localisation from a synthetic ECM. Our goal is to engineer a microenvironment based on PEA coated surfaces that can favour this synergistic mechanism.

Several works in the literature have shown matrix bound GFs elicit prolonged activation of cell GF receptors and reciprocal responses on adhesion integrins, resulting in enhanced biological function with lower doses used, and therefore more efficient compared to soluble administration [8] [12]. These works have also shown that presentation of GF from the natural ECM happens through specific binding sites, like the heparin II binding region of fibronectin (FNIII12-14) [13] that is able to bind to many different GFs. FN domain FNIII9-10 contains the well-known RGD site that promotes cell adhesion, and both domains are jointly available when FN naturally 
unfolds by cell-ECM interactions and rearrangement. Moreover, the arrangement of FN by cell-ECM interactions can be mimicked by synthetic surfaces that can drive fibronectin into a fibrillar conformation that exposes its functional domains [6]. When GFs are presented bound to the ECM, by the GF binding domain in FN, interaction between integrins and GF receptors can result in a 'crosstalk' that stimulates in synergy cell adhesion and activation of cell GF receptors. A schematic diagram of these mechanism is presented in Figure 1. A full page version of Figure 1 is displayed in Appendix 8.5.

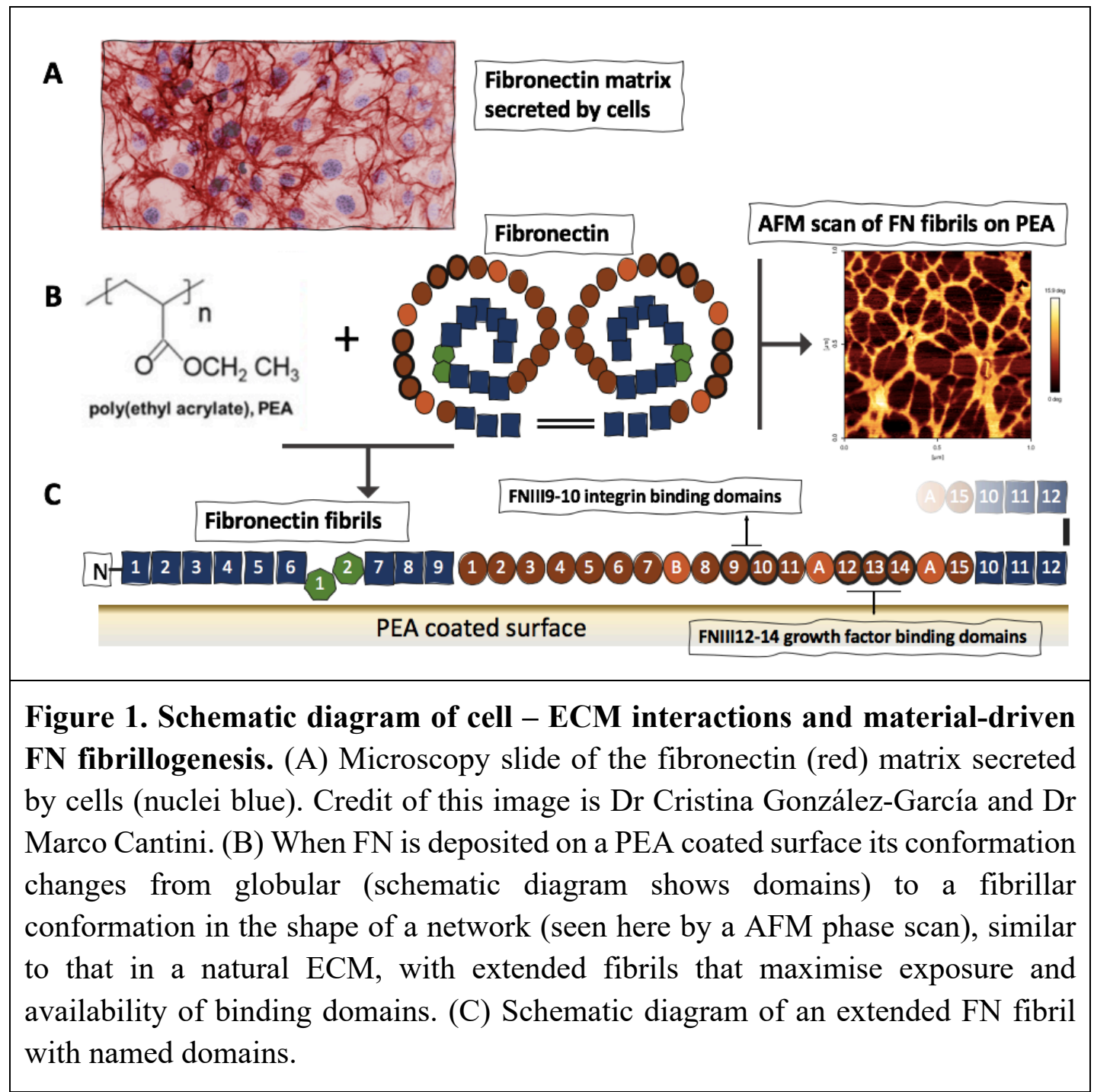

\subsection{Coating with polymers of biomedical interest}

A perfect synthetic implant would be one to provide support with optimal mechanical and structural properties, merge with the damaged tissue and deliver biological cues to foster the natural healing processes. With these goals, coatings on orthopaedic implant surfaces for instance can be used to improve the implant performance, following a variety of different strategies that look to recreate particular aspects of the bone regenerative microenvironment. These surface modifications to improve cell 
interactions are sometimes already seen in available commercial products, but many are yet being explored in research or clinical phases

A variety of materials from synthetic or natural origin are used directly for their own osteogenic effect, as bioactive coatings that artificially mimic the ECM and can control cell behaviour, or they are used as carriers of functional molecules [14]. The first strategy usually involves surface modifications to present ligands of interest and adjusting their biochemical and biophysical properties to better sustain cell biology. The second strategy involves tuning of chemical (swelling, cross-linking) properties to carry and deliver specific molecules.

\subsubsection{Polymers as active coatings}

The strategy of engineering materials and their surfaces for enhanced osseointegration and bone repair is used in one way or another in most implant biomaterials, metal, polymer or ceramics-based, in similar conceptual fashion. Several comprehensive reviews exist in the literature [15] [16]. A variety of well-known classic techniques are commonly used to prepare polymer-coated surfaces, in general either chemical or physical deposition processes such as dip coating, spin coating, plasma polymerization, electrospinning, spray coating, and others. Most of these techniques can achieve useful films for particular biological applications, although drawbacks relating to lack of film stability, materials available to the technique, reproducibility and control over thickness are usually reported.

The biochemical and biophysical properties of polymers are able to influence cell biology, including cell adhesion, orientation, migration and differentiation [14]. Polymers as bioactive coatings have been developed and tested in orthopaedic applications as antimicrobials, to improve the mechanical fixation of implants and with an intrinsic osteogenic functionality. On top of their chemical properties, researchers have explored the control of the surface topography of materials with biomedical interest to provide biophysical cues to guide cellular functions at the cellmaterial interface [17] [18].

Presentation from surfaces of bioligands, surface functional groups and charge of polymer can play an even more significant role in cell - material interactions. RGD sequences and ECM proteins or fragments of them are often used to make biomaterials conducive for cell attachment. A coating with functional motifs can improve cell adhesion and effectivity of delivered or endogenous molecules like growth factors, both critical in many applications.

Hoyos-Nogués et al [19] for instance combined integrin (RGD) and heparin (KRSR)binding peptides, mimicking synergistic cell-membrane receptor binding, to improve osteoblastic functions in vitro. The peptides were grafted covalently to titanium surfaces to demonstrate that this strategy can enhance bioactivity of medical implants.

Gandavarapu et al. [20] used phosphate functional groups incorporated into PEG and the gels pre-incubated with serum were able to support hMSCs even in serum free 
medium, promoting osteogenic differentiation. Blocking assays with antibodies attributed this effect to collagen and fibronectin adsorbed matrix components on the surfaces and $\beta 1$ and $\beta 3$ integrins on cells.

Coatings of ECM proteins are also specifically examined to improve the delivery and presentation of growth factors, or their effect from endogenous sources. Gigliobianco et al [21] for instance prepared scaffolds with polymer materials in layers combined with heparin and studied their ability to bind vascular endothelial growth factor (VEGF) and induce angiogenesis.

However, interactions between growth factors and the biomaterial carriers are usually not specific, and high supra-physiological doses of GF have to be used to trigger a regenerative response. Works on the mechanisms that regulate GF and ECM protein interaction have shown how integrin and GF receptors cooperate to produce enhanced GF signalling, and how this knowledge about synergistic GF/integrin signalling can be used to engineer microenvironments that target simultaneous integrin and GF receptor engagement. With an adequate microenvironment design, this strategy maximises GF effects in vitro (e.g. in terms of stem cell differentiation) but also tissue repair in vivo (e.g. bone regeneration and wound healing) [8].

Ultimately researchers are looking to understand how particular chemical groups or surface features in a material influence processes in cell - material interfaces, in particular differentiation and regenerative mechanisms. Advances in synthesis and characterization of new coating techniques have been explored with increased interest to develop well-defined architectures and specific functional coatings, such as polymer brushes and self-assembled monolayers [22] [23]. Prof Salmeron-Sanchez's lab in Glasgow for instance have developed PEA polymer brushes on PLLA substrates by an atomic transfer radical polymerization technique [24]. The PEA brushes trigger FN organization into nanofibrils, enhancing adhesion and differentiation of $\mathrm{C} 2 \mathrm{C} 12$ cells. Several labs are working with similar strategies to fine-tune functional polymer surfaces, testing them on stem cells in vitro, to ascertain how cell behaviour, in particular osteogenic differentiation, is affected by different terminal groups or varying wettability.

\subsubsection{Polymer coatings as carriers for controlled delivery}

Polymer coatings as carriers of functional molecules are a powerful biomedical engineering tool, to allow and control release of therapeutic agents including osteogenic factors. Implant coatings have been developed to release therapeutics such as statins, antibiotics, growth factors, cytokines, and others [25]. Release of antibiotics from the implant surface for instance is an active field of research to mitigate or prevent the consequences of orthopaedic infections [26]. Osteogenic factors have been found to be critical in the regulation of the bone regeneration process and the engineering of their interaction with matrices and surfaces is a key field of research for the next generation of tissue engineering applications [27], [28]. There is a variety of developed technologies and ongoing research to devise coatings for implants for 
growth factor delivery, where the polymer works as a carrier of the bioactive molecules or as a functional surface from where they are absorbed and presented. The efficiency of growth factors depends a lot on the selection of suitable carriers. Bone morphogenetic proteins for instance are currently only approved by regulatory agencies to be used with collagen carriers in spinal fusion, oral and maxillofacial surgery and also in the repair of long bone defects. Many research works have explored the use of different types of carrier materials for BMPs: natural and synthetic polymers, calcium phosphate and ceramic-polymer composite materials. In the case of polymers, for instance multi-layered coatings (chitosan, hydroxyl apatite, polyacrylic acid) have been used to release BMP-2 from titanium scaffolds [29]. In a different work, films consisting of 100 successively deposited tetralayers of polyelectrolyte (Poly (b-aminoester) 2,) capable of delayed release of BMP-2 were fabricated on commercially available polycaprolactone/b-tricalcium phosphate copolymer blend 3D-printed scaffolds [30]. Other examples related to delivery of osteogenic growth factors are works by Almodovar et al. [31] that used layer-by-layer (LbL) deposition techniques to manufacture films with spatial control of gradient concentration of BMP-2 and BMP-7 and test their effect on $\mathrm{C} 2 \mathrm{C} 12$ myoblasts, and by Caridade et al. [32], that prepared polysaccharides chitosan / alginate free-standing membranes with a similar dipping LbL technique that could be tuned (cross-linked) to an optimal release profile of BMP-2 with myoconductive and osteoinductive effects in in vitro and in vivo scenarios.

\subsubsection{Coating with plasma polymerised materials}

Plasma polymerisation of a monomer by radio frequency glow discharge, allows the deposition of highly tuneable polymer films onto a variety of substrates such as metals, ceramics and polymers. Academic institutions across the world use a variety of plasma chambers in their labs. Commercial equipment is available from specialised manufacturers. Built in house equipment is however still often used, following design constraints and prototypes originally set out in the literature around forty years ago [33] [34], for its flexibility and cost advantages with the drawback of differences in performance between equipment, even when based in the same design. Deposition rate, plasma pressure, film uniformity, amongst others are generally specific to every individual chamber.

Many works in the literature describe in detail the parameters that control the nature of plasma polymerization and deposition of organic thin films, as this technology has been used in research and industry labs for several decades now [35]. The general goal has been to improve the surface properties of a substrate material or to use the physical properties of the coating itself, originally in the mechanical, electrical and optical industries, and more recently in biomedical applications too.

Plasma polymerization produces coatings with properties fairly independent of the substrate after a sufficient time of treatment. At the early stages of plasma, polymer growth is however not completely independent of the properties of the substrate. 
Vasilev et al. [36] produced plasma polymerized coatings on gold and organic surfaces from two nitrogen (amine)- and two carboxyl-containing monomers to ascertain the role of the substrate in the early stages of plasma. Deposition rates and XPS spectra form this work showed that the thickness and chemistry of the coatings are different for different substrates during the first seconds of treatment, but after a few nanometres of coating has been deposited, the coating growth and its chemical nature becomes substrate independent, dependent only on the monomer used and parameters of the plasma.

Applications of plasma polymerization in biomedical applications have been increasingly explored as targeted research works have shown that plasma polymerized coatings can, by themselves, critically improve the biological functionality of a material substrate. Here we note the immense amount and quality of work done in recent years in applications related to enhancing bone integration and tissue regeneration for dental and orthopaedic surgery, where many strategies to engineer surfaces of implant by tuning their chemistry, mechanical properties, or/and topography rely on the use of plasma polymerized coatings.

For instance, Gabler et al [37] used plasma-polymerized allylamine (AAm) and plasma-polymerized ethylenediamine (EDA) to coat titanium alloy implant surfaces, showing enhanced adhesion of human bone cells in vitro and bone ongrowth in vivo in a rat model. Liu et al [38] also studied surfaces coated with plasma polymerized AAm, acrylic acid, 1,7-octadiene, and ethanol precursors and showed that AAm coatings in particular promote osteogenic differentiation of human adipose-derived stem cells. Zheng et al. [39] used plasma polymerization of acrylic acid to carboxylate a poly(etheretherketone) (PEEK) surface, with control of density of functional groups on the PEEK surface. The coated surfaces showed improved osteoblasts adhesion, spreading and proliferation. PEEK is usually bioinert and although its mechanical properties make it suitable for orthopaedic implants it has poor osteoblast adhesion without any surface modification. Cools et al. [40] deposited methyl methacrylate based coatings on Titanium surfaces, to improve the Ti-PMMA bone cement interfacial adhesion, up to a $50 \%$ in adhesive force and maintaining stable adhesive properties over time. The same lab recently published a focused review [41] on the use of acrylic acid plasma polymerization in biomedical applications, concluding that a successful deposition of $\mathrm{COOH}$-rich stable coatings is able to improve cell growth, proliferation and differentiation, but that the mechanisms behind the cell-material interactions still needs to be explored in more detail. 



\section{Overall aim and specific objectives}

Our aim in this work has been to develop and characterize coating techniques based on the surface deposition of polymer PEA that improve the functional interaction between living tissue and biomaterials. Bulk PEA was known previous to this work to drive FN fibrillogenesis, and thus enhance cell - matrix interactions that involve growth factors, allowing the development of clinical applications with their use in low physiological doses.

With the plasma polymerization and spray techniques developed in this work we have aimed to enable the use of PEA as a coating on surfaces of structures such as scaffolds and implants, that can't be coated or made with bulk PEA.

In particular, in this thesis we have pursued the following specific objectives:

i Develop and characterize a custom-made low power radio frequency glow discharge equipment and a plasma polymerization protocol to coat surfaces with plasma polymerized ethyl acrylate monomer. We have aimed to confirm that our chosen design for a plasma polymerization reactor (low pressure, low power) allows a fine control of the chemical integrity and the thickness of the coating, even at sub-micron scales. We speculate that if feasible with our plasma reactor, thin coatings can be successfully broken into pieces and cleared off by cells and their biological activity. This would allow therefore the use of the coating in applications where the substrates are biodegradable and the whole biomaterial system is required to be biodegradable.

i.A Our first goal here has been to test and set-up the right equipment that best satisfies requirements for running a glow discharge at a low power range, uniformity of coating over large samples and ease of use with a high number of samples. We have also looked to optimise the practical operation of this equipment in the lab, with a variety of samples.

i.B Then our goal for the plasma polymerized coatings has been to systematically evaluate process conditions and their impact on the coatings:

i.B.1 to characterize the thickness of the coatings, and how it is controlled with the polymerization parameters,

i.B.2 confirm that we are able to produce uniform very thin coatings of only a few tens of nanometres, and

i.B.3 characterise how fibronectin behaves when coated onto them.

i.C Additionally, we have aimed to confirm that the technology is useful to cover 3D structures, in particular

i.C.1 rough surfaces with functional roughness or topographies and 
i.D porous structures such as scaffolds, with a uniform coating with functional properties, characterise the effect of the coating on the degradation rate of a biodegradable substrate (PCL).

ii Develop and characterize a custom-made compressed air spray equipment to coat surfaces with PEA in a volatile solvent. This a very simple and low-cost technology that allows to use PEA from radical polymerization in the lab or commercial sources.

ii.A We aimed to set-up and optimise protocols for the two spraying installations, one with a high-volume airgun and a second with a more precise airbrush.

ii.B Characterise sprayed coatings with spraying parameters, i.e. thickness, partial/full coating distribution, and degradation in water.

ii.C Confirm the conformation of fibronectin on sprayed surfaces with AFM

ii.D Confirm the utiliy and limits of the spraying technique to coat $3 \mathrm{D}$ structures with pores.

ii.E Characterise the effect of the sprayed coating on the degradation rate of a biodegradable substrate (PCL). 


\section{Materials and methods}

\subsection{Equipment set-up and materials}

\subsubsection{Plasma equipment set-up}

A custom-built capacitively inducted plasma reactor was used to polymerise EA monomer onto microscopy coverslip glasses via plasma polymerisation. Our system is a low-pressure plasma T-shaped reactor made of borosilicate (De Dietrich) and stainless-steel end plates (custom made in lab workshop) sealed with Viton O-rings with a volume of around $15 \mathrm{~L}$. Vacuum was produced by a rotary pump or by a scroll pump (both BOC Edwards), with operating experiment pressures from $5.0 \times 10^{-3}$ to $6.0 \times 10^{-1}$ mbar. The plasma was initiated via two capacitively coupled copper band ring electrodes situated outside of the reactor chamber and connected to a radio frequency power supply (Coaxial Power System Ltd.) that works at $13.56 \mathrm{MHz}$ up to 300Watt. The monomer pressure was controlled via speedivalves (BOC Edwards) and monitored with a pirani gauge (Kurt J. Lesker).

Set-up considerations about working with EA monomer in plasma polymerization experiments. EA is not very volatile compared to other monomers and stabilization of EA flow and pressure in the reactor requires good control of vacuum and of operation of vial and inlet valves into the reactor chamber. In our system EA monomer was poured into a custom-made glass vial usually in an amount needed for several experiments and kept in a dedicated fridge while not in use. Before an experiment the vial was attached to the inlet of the reactor and left to warm to room temperature for around ten minutes before staring a deposition experiment. After finalising an experiment, the vial with remaining monomer was sealed and stored for upcoming experiments. The monomer gas phase pressure inside the reactor was predetermined and adjusted in a systematic manner for all experiments. The optimal working pressure for our experiments was chosen as the highest that was able to spark and maintain a plasma while reflected power was minimum or zero. This pressure depends on several parameters, i.e. the applied RF power, the physical and electrical characteristics of the specific reactor, and also on the nature of the plasma phase in the reactor (air, oxygen, monomer, etc).

Our full experimental operating protocol is included in appendix 4.2. A detailed discussion of reactor design and assembly issues, and of considerations to facilitate the polymerisation of EA are discussed in section 3.1.

\subsubsection{Spray equipment set-up}

We built two custom made air spraying installations in our lab: (i) A simple cage for a high-volume airgun and (ii) a clamp holder with a tilted target for a dual action low 
volume airbrush, to deposit PEA from solutions in toluene. Both systems were used inside a laminar flow hood.

The air gun spraying set-up was assembled around a cheap and simple to use airgun that was positioned inside a custom-built closed cage with pre-fixed positions at 40 $\mathrm{cm}, 50 \mathrm{~cm} \mathrm{\&} 70 \mathrm{~cm}$ from nozzle to target surface. Pressurized air was supplied by a compressor and all experiments were performed at 0.8 to 1.1 bar of air pressure.

The airbrush spraying set-up consisted of a commercial airbrush held in place with a clamp at the right height towards a tilted target for samples.

\subsubsection{Substrates and materials}

Circular $12 \mathrm{~mm}$ diameter microscopy cover glasses (borosilicate glass D263 ${ }^{\mathrm{TM}} \mathrm{M}$, Marienfeld $\mathrm{GmbH} \& \mathrm{Co} . \mathrm{KG}$, Germany) were used as reference substrates for the polymer coating, both in plasma polymerisation and spraying experiments.

We used ethyl acrylate monomer from Sigma Aldrich (St. Louis, MO) for our polymerisation experiments.

We used commercial and in-house polymerised PEA for spray coating experiments and for our spin coated control samples respectively. In house bulk PEA was obtained via polymerization of EA using $1 \%$ benzoin (Sigma Aldrich) as a photoinitiator and dissolved in toluene (Sigma Aldrich) for spin coating applications. Commercial PEA (Sigma Aldrich) is available dissolved in toluene already, at a concentration of around 19 to $21 \%$ depending on the batch, that we reduced for our spray coating experiments to $8 \%$ or lower.

Preparation of glass substrates for plasma experiments. Glasses were cleaned by sonication in ethanol for 30 mins and dried in a lab oven prior to use. As a first stage of every plasma experiment, samples were exposed to air plasma for 5 minutes at $100 \mathrm{~W}$ of RF incident power to ensure removal of any residual organic matter.

Spin coated control samples. Spin coated PEA samples were prepared on the same $12 \mathrm{~mm}$ cover glasses from a 4\% solution in toluene of bulk PEA polymerised in our lab. Spin coating was operated at a speed of $3000 \mathrm{rpm}$ with an acceleration of 3000 rpm s$~^{-1}$ for $30 \mathrm{~s}$ and vacuum dried at $60^{\circ} \mathrm{C}$ for $2 \mathrm{~h}$ to extract excess toluene.

Samples for roughness conservation experiments after plasma polymerisation coatings. Amorphous and crystalline polylactic acid (PLA) (Purasorb PL 18, Corbion, Amsterdam) surfaces were prepared for roughness conservation experiments. Spin coated PLA samples were prepared on $12 \mathrm{~mm}$ cover glasses from $2 \%$ solution in chloroform (Sigma, St. Louis, MO) of bulk PLA (amorphous PLA). To obtain crystalline PLA surfaces, spin coated samples underwent thermal treatments. To yield a crystalline PLA surface with small spherulites, samples were put in an oven at 200 ${ }^{\circ} \mathrm{C}$ for 5 minutes (melting stage), then at $75{ }^{\circ} \mathrm{C}$ for 6 hours (nucleation stage), and finally at $110{ }^{\circ} \mathrm{C}$ for 2 hours (crystallization stage). This produces a crystalline PLA with high roughness. To yield a crystalline PLA surface with big spherulites, samples 
were put in an oven at $200{ }^{\circ} \mathrm{C}$ for 5 minutes, and then at $110{ }^{\circ} \mathrm{C}$ for 2 hours. This produces a crystalline PLA with lower roughness.

Scaffolds. Microporous PLA (Purasorb PL 18) scaffolds of different sizes were produced with a combination of freeze extraction and porogen leaching techniques. We used poly(ethyl methacrylate) (PEMA) spheres of Elvacite-2043 acrylic resin (Lucite International Speciality Polymers and Resins Ltd) as porogen. First, 10\% weight PLA was mixed with 1,4-Dioxane anhydrous (Sigma Aldrich) and stirred until complete dissolution. PLA and dioxane solutions were then poured into Teflon moulds and mixed with the PEMA porogen spheres in 1/1 solution porogen weight ratio and immediately frozen in liquid nitrogen. Cold ethanol at $-20 \mathrm{C}$ was poured on the frozen samples in order to dissolve the crystallized dioxane. Dioxane extraction was conducted in a cold ethanol bath at $-20 \mathrm{C}$, changed ethanol twice a day for three days, until dioxane had disappeared from the sample. The extraction of the PEMA porogen spheres was carried out with ethanol at $40 \mathrm{C}$ under slow stirring; ethanol was changed until the PEMA was removed from the structure. After extraction, the scaffolds were dried in air atmosphere for $24 \mathrm{~h}$ and then in vacuum to constant weight, first at room temperature and then at $40 \mathrm{C}$. The scaffolds were cut into cylinders of 6 $\mathrm{mm}$ diameter and about $4 \mathrm{~mm}$ high.

Polycaprolactone (PCL) scaffolds were fabricated with an extrusion-based 3D printer (3D Discovery, regenHU, Villaz-St-Pierre, Switzerland) with a printing nozzle diameter of $330 \mu \mathrm{m}$. The scaffold architecture was designed using computer-aided design software (BioCAD, regenHU, Villaz-St-Pierre, Switzerland). The scaffold was designed with a fibre spacing of $660 \mu \mathrm{m}$, a slice thickness of $280 \mu \mathrm{m}$, a $0^{\circ} / 90^{\circ}$ laydown pattern, and dimensions of $30 \mathrm{~mm} \times 30 \times \mathrm{mm} \times 3.36 \mathrm{~mm}$. The printing parameters used are deposition velocity of $20 \mathrm{~mm} \mathrm{~s}-1$, a material chamber temperature of $90^{\circ} \mathrm{C}$, an extrusion pressure of 6 bar, and a screw rotation velocity of $15 \mathrm{rpm}$.

\subsection{Characterization techniques}

\subsubsection{Atomic force microscopy (AFM)}

Atomic force microscopy was used for imaging and characterising the surface topography of plasma deposition surfaces before and after FN coating on dried samples in air. For fibronectin (FN) (R\&D Systems) coated samples: After FN adsorption, samples were washed with water and dried with nitrogen flow before imaging.

A JPK Nanowizard 4 (JPK Instruments) apparatus was used for imaging in tapping mode using antimony-doped Si cantilevers with a nominal resonant frequency of 75 $\mathrm{kHz}$ (MPP-21220, Bruker). The phase signal was set to 0 at a frequency $5-10 \%$ lower than the resonant frequency. Height and phase images were acquired from each scan. The JPK Data Processing software versions 5 and 6 were used for image analysis. 
For an estimation of the thickness of the coatings, a thin scratch was performed manually and gently with a sharp blade onto the surface of coated glass substrates, to expose the underlying substrate. The surface was then viewed under optical microscopy to identify an optimal and clean area in the scratch, and the area across the cut scanned. The thickness of the polymer coating was estimated measuring the difference in height profile between the scratched and unscratched area, as an average of at least five measurements.

\subsubsection{Water contact angle (WCA)}

Water contact angle measurements were taken on all coated surfaces before and after FN coating. Static contact Angles (SCAs) were measured by dropping $3 \mu \mathrm{L}$ drop of deionized water on to the surfaces using Theta optical tensiometer (Biolin Scientific, Stockholm, Sweden). The stability of the coatings was also checked up to 14 days after undertaking the plasma/spin coating treatment.

\subsubsection{X-Ray Photoelectron Spectroscopy (XPS)}

$\mathrm{X}$-ray photoelectron spectroscopy was used to identify the surface chemical composition of coated samples. All X-ray photoelectron spectra were obtained at the National EPSRC Users' Service (NEXUS) at Newcastle University (found at: http://www.ncl.ac.uk/nexus/). Each sample was analysed at three points with a maximum beam size $(400 \mu \mathrm{m} \times 800 \mu \mathrm{m})$ with a K-alpha XPS apparatus (Thermo Scientific) equipped with a monochromatic $\mathrm{Al} \mathrm{K}$-alpha source for carbon, oxygen and overview spectra. X-ray energy was $1486.68 \mathrm{eV}$ at a voltage of $12 \mathrm{kV}$, current of 3 $\mathrm{mA}$ and power of $36 \mathrm{~W}$. Spectra analysis and curve fitting were performed using CasaXPS software version 2.3.16.

\subsubsection{Protein adsorption and domain exposure assays}

Adsorption of FN on surfaces. FN was coated on surfaces of interest at a concentration of $20 \mu \mathrm{g} / \mathrm{mL}$ in DPBS unless otherwise stated. A $200 \mu$ droplet of solution was deposited to cover the surface of a $12 \mathrm{~mm}$ glass coverslip, and let to adsorb for 10 minutes on each sample. After washing once with DPBS and once with miliQ water, samples were dried for a few seconds with nitrogen flow prior to be scanned with AFM to characterise the behaviour of FN.

Micro Bicinchoninic acid protein quantification assay (BCA). The density of adsorbed protein was determined by measuring the amount of non-adsorbed FN. A standard curve was created via serial dilutions of an FN stock of known concentration. Samples were coated for $1 \mathrm{~h}$ and the remaining FN solution was transferred to 96-well plates, where the bicinchoninic acid working reagent was added (Thermo Fisher Scientific, Waltham, MA). The plate was then agitated and incubated at $37{ }^{\circ} \mathrm{C}$ for 2 h. The absorbance was read at $562 \mathrm{~nm}$ with a Tecan NanoQuant Infinite M200 Pro plate reader (Männedorf, Switzerland). 
Exposure of specific fibronectin domains. ELISA was performed to assess the exposure of specific domains on the FN molecule. After surfaces had been coated with $20 \mu \mathrm{g} \mathrm{mL}-1 \mathrm{FN}$ in DPBS for $1 \mathrm{~h}$, they were blocked for $30 \mathrm{~min}$ with $1 \%$ bovine serum albumin (Sigma-Aldrich) in DPBS. Subsequently, primary antibodies for the FN(III910) domain (HFN7.1, mouse monoclonal, 1:330, Developmental Studies Hybridoma) and FN(III12-14) domain (P5F3, mouse monoclonal, 1:2000, Santa Cruz Biotechnology) were added onto the surfaces and incubated for $1 \mathrm{~h}$. The surfaces were thereafter washed $3 \times 5$ min with $0.5 \%$ Tween 20 in DPBS. A horseradish peroxidase (HRP)-conjugated anti-mouse antibody (1:10000, Thermo Fisher) was then added onto the surface and incubated for $1 \mathrm{~h}$ in the dark, followed by washing for $3 \times 5 \mathrm{~min}$ with $0.5 \%$ Tween 20 in DPBS. Afterwards, a substrate solution (R\&D Systems) was then added onto the surfaces and the samples were incubated in the dark for $20 \mathrm{~min}$, followed by the addition of a stop solution (R\&D Systems). The absorbance of the coloured solution was read at 450 and $540 \mathrm{~nm}$ and the data were used to determine the relative exposure of the $\mathrm{FN}$ domains. All procedures were performed at room temperature.

Quantification of BMP-2 adsorption. The density of adsorbed BMP-2 was determined by measuring the amount of non-adsorbed BMP-2 that remained in the supernatant via sandwich ELISA (R\&D System) following the manufacturer's instructions.

Briefly, in this process, ELISA plates were coated with the capture antibody before they were blocked with the bovine serum albumin for 1 hour. After appropriately diluted supernatants were added, bound BMP-2 was detected with biotinylated antihuman BMP-2 antibody. Streptavidin conjugated horseradish peroxidase was then added to the plates. Enzyme substrate was treated for 20 minutes before the reaction was stopped by adding an acidic solution. Absorbance was measured at $450 \mathrm{~nm}$ with wavelength correction at $570 \mathrm{~nm}$. The standard curve was calculated using a fourparameter logistic (4-PL) curve fit. The amount of adsorbed BMP-2 was calculated from a standard curve based on known concentrations of BMP-2.

\subsubsection{Differential scanning calorimetry (DSC)}

DSC heating scans (PYRIS-DSC 8000, Perkin Elmer) were conducted to detect and measure glass transition temperature in plasma polymerised materials and compare with bulk references.

The scans were run with a $\mathrm{N} 2$ flow of $20 \mathrm{ml} / \mathrm{min}$ and the following heating and cooling program: (1) $30{ }^{\circ} \mathrm{C}$ to $70{ }^{\circ} \mathrm{C}$ at a heating rate of $20{ }^{\circ} \mathrm{C} / \mathrm{min}$, (2) hold for $2 \mathrm{~min}$ at 70 ${ }^{\circ} \mathrm{C}$, (3) from $70{ }^{\circ} \mathrm{C}$ to $-80{ }^{\circ} \mathrm{C}$ at a cooling rate of $20{ }^{\circ} \mathrm{C} / \mathrm{min}$, (4) Hold for $5 \mathrm{~min}$ at -80 ${ }^{\circ} \mathrm{C}$, and finally from $-80^{\circ} \mathrm{C}$ to $70^{\circ} \mathrm{C}$ at a heating rate of $20^{\circ} \mathrm{C} / \mathrm{min}$. 


\subsubsection{Degradation of PCL scaffolds}

The degradation of PCL scaffolds was characterised following standard ASTM F1635, "Standard Test Method for in Vitro Degradation Testing of Hydrolytically Degradable Polymer Resins and Fabricated Forms for Surgical Implants".

Specimens of each scaffold group were accurately weighed, sterilized for 20 minutes with UV light (10 minutes each side), then placed in 24 well plates and immersed in DMEM cell culture medium containing an added $1 \%$ of $\mathrm{P} / \mathrm{S}$ antibiotic and $0.1 \%$ of fungizone. DMEM contains phenol red, a $\mathrm{pH}$ indicator to visually control the $\mathrm{pH}$ of the media. Samples were incubated $\left(37^{\circ} \mathrm{C}, \mathrm{pH} 7,4\right)$.

Media loss by evaporation was checked weekly and fresh media added to the well plates as needed. All weighing measurements were performed in triplicate and the results shown are the average of 3 replica. After the periods of evaluation, the samples were removed from the buffer, gently dried with a filter paper, washed three times with deionized water, dried in an oven for 60 minutes at $60^{\circ} \mathrm{C}$ and stored in a vacuum desiccator for 4 days at room temperature, and then weighed. 


\section{Results and discussion. Plasma coatings.}

\subsection{Assembly of a plasma polymerization reactor}

We designed and built several low-pressure capacitively coupled plasma reactors looking to test and chose the design best suited for the production of coated 3D samples (scaffolds, porous granules, tubes). Critical requirements considered to select the reactor considered in particular its planned use beyond this work to produce samples for in vitro and in vivo experiments in our lab's line of research on functional materials to deliver growth factors. The reactor needed to accommodate and homogeneously coat batches of samples where each sample is of at least several centimetres in their main dimension. Testing and sample production needed to be as easy as possible to scale up production to a higher number of samples for planned research work and preclinical stages.

For our main plasma polymerization installation, we built a plasma reactor with outer ring electrodes and with a side access port. We chose it as our main reactor for its ease of use, control of plasma spark, ease of access to samples, easier cleaning, and better safety to open and close the reactor.

Previously we built and tested two other prototypes, different sized variations of a design with a cylindrical reactor, a top lid and inner parallel plate electrodes (Figure 2 ). Both these prototypes proved to be cumbersome and unpractical, in particular when getting samples in and out of the reactor, a critical requirement to comply with the number of experiments and size of samples planned for this work and elsewhere.

We tested all three prototypes for vacuum and for plasma spark but only worked with monomer with the main one. For it, we assessed range of monomer plasma pressure, rate and homogeneity within the sample area of material deposition, and control of thickness and chemical integrity of the functional coatings.

For the design and construction of these plasma reactors we have followed previous working designs of lab plasma chambers used in the literature to polymerise monomers as discussed in the introductory section.

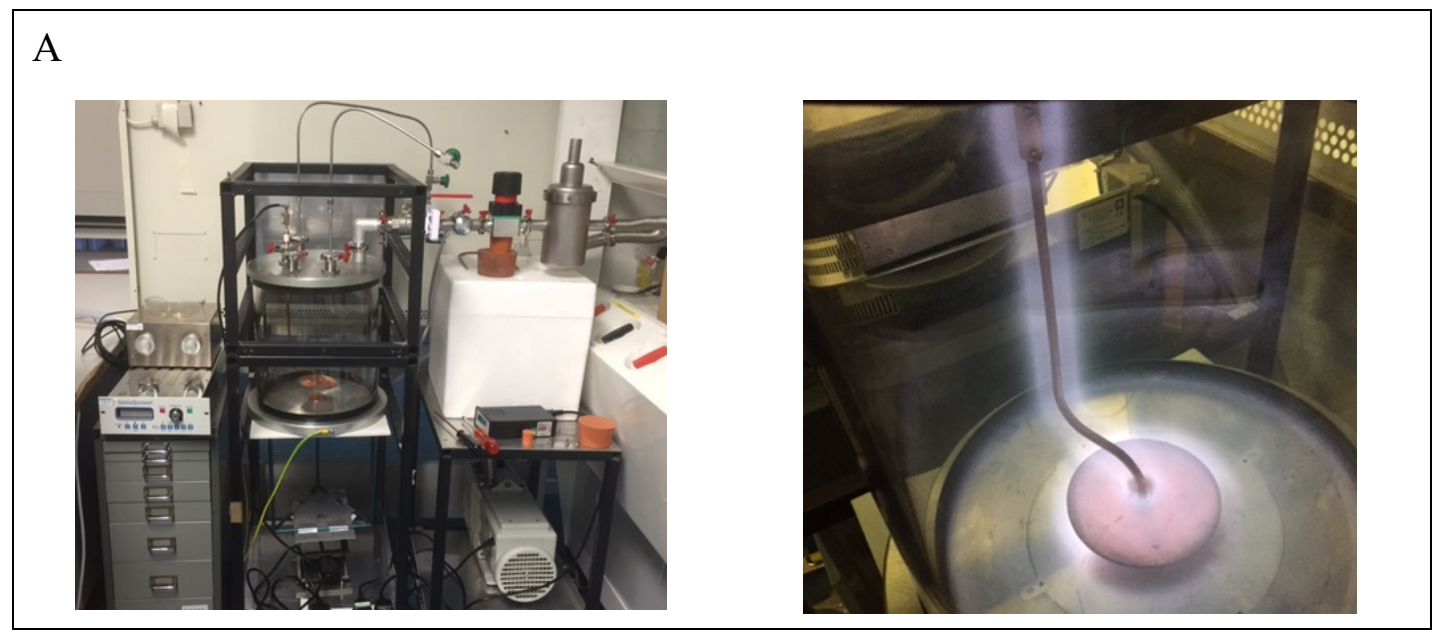


B
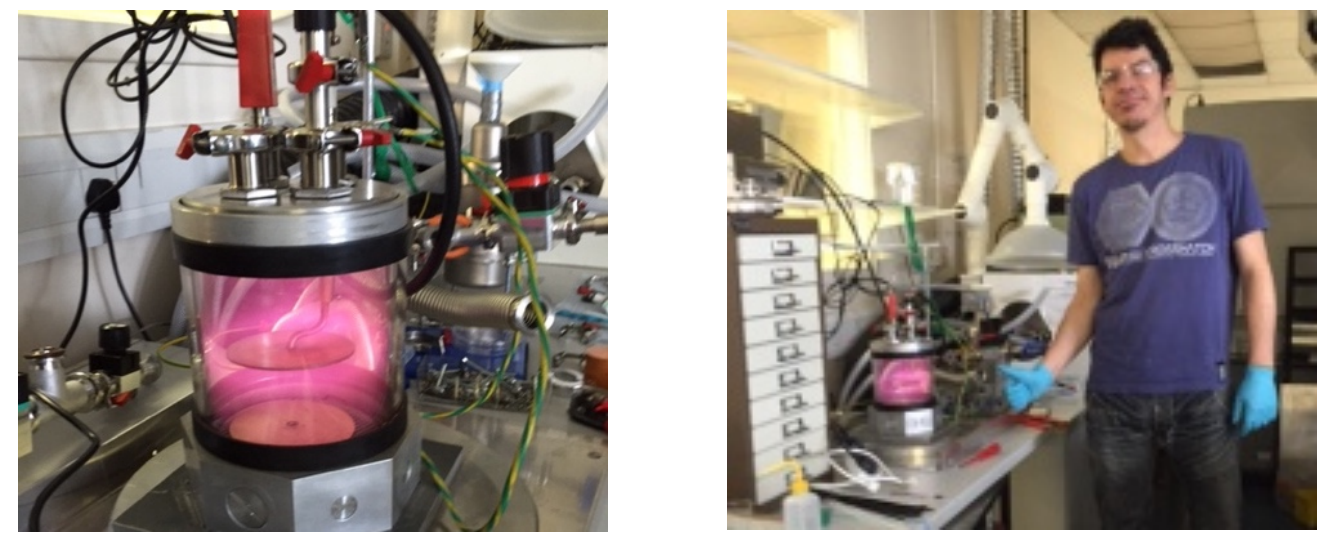

C
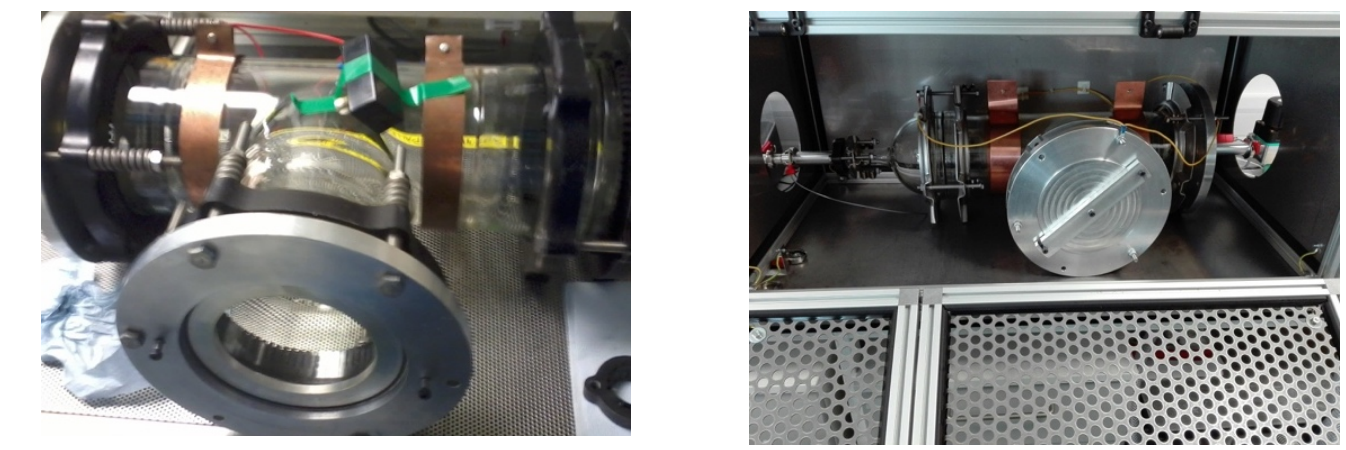

$\mathrm{D}$
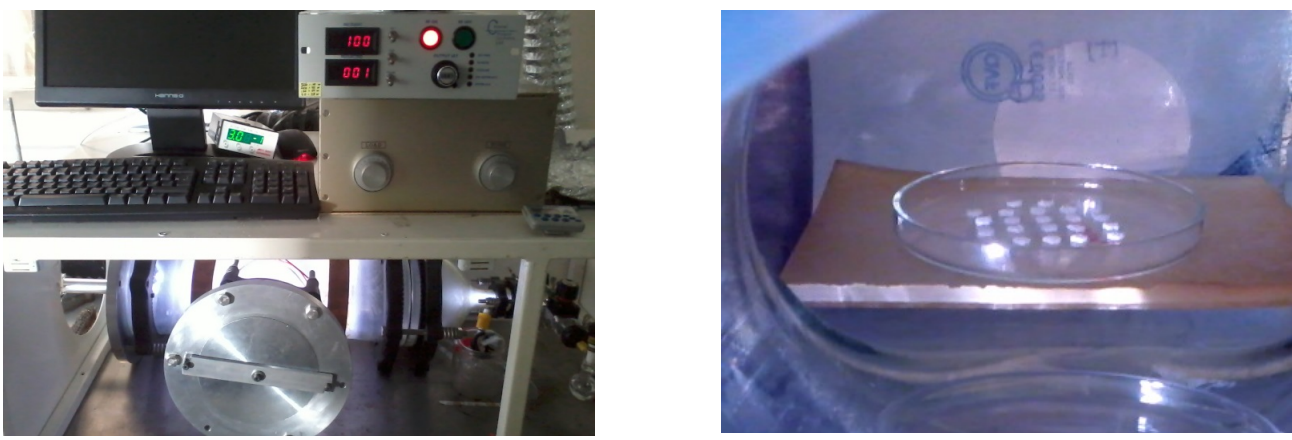

Figure 2. Views of the three plasma polymerization reactor designs assembled in this work. (A) Prototype of a cylinder shaped reactor with parallel plate electrodes and a volume of 20L. (B) Similar design with a reactor volume of 1L. (C) Prototypes of T-shaped reactor with outer ring electrodes, showed left is our first prototype used for all the experimental data presented in this work, and showed right is our second prototype, assembled to be used in related research. (D) A monomer polymerization test with white plasma glow discharge, with view from the side port of the chamber (D, left), and view of scaffold samples placed on the sample holder position (D, right). 


\subsubsection{Main reactor}

The reactor design fully characterised in this work for the plasma polymerisation of EA coatings is a custom-built T-shaped reactor with outer ring electrodes and a volume of nearly $15 \mathrm{~L}$.

\subsubsection{Reactor design}

The design chosen is a capacitively coupled plasma reactor, with a T-shaped vacuum chamber custom built with glass parts and flanges from vacuum specialist supplier De Dietrich Gmbh and stainless-steel end plates custom made in our workshop. This is a low pressure and low power plasma reactor design with outer ring-shaped electrodes.

We tested two types of pumps to produce vacuum in our installation: a more powerful scroll pump and a cheaper and readily available rotary pump (both pumps BOC Edwards). Operating experiment pressures have been from $6.0 \times 10^{-1}$ to $5.0 \times 10^{-3}$ mbar. The scroll pump allowed for better control and stability of pressure during the monomer plasma and was used for most of the results reported in this work. The rotary oil pump was used in the first calibration and testing of all the tested reactors. Both pumps showed significantly different working pressures to achieve plasma spark and during the monomer polymerisation stage. Also, deposition rate of polymer was significantly higher with the lower plasma pressure scroll pump, which highlights the sensitivity of the performance of this installation to seemingly minor modifications.

In this design the plasma was initiated via two capacitively coupled copper band ring electrodes situated outside of the reactor chamber and connected to a radio frequency power supply (Coaxial Power System Ltd.) that works at $13.56 \mathrm{MHz}$ up to a power of $300 \mathrm{~W}$. The monomer pressure was controlled via speedivalves (BOC Edwards) and monitored with a pirani gauge (Kurt J. Lesker).

Schematics of this system and pictures of the installation are presented in Figure 3.

Detailed schematics, references for the components used and our operating instructions are included in appendices at the end of this document. 


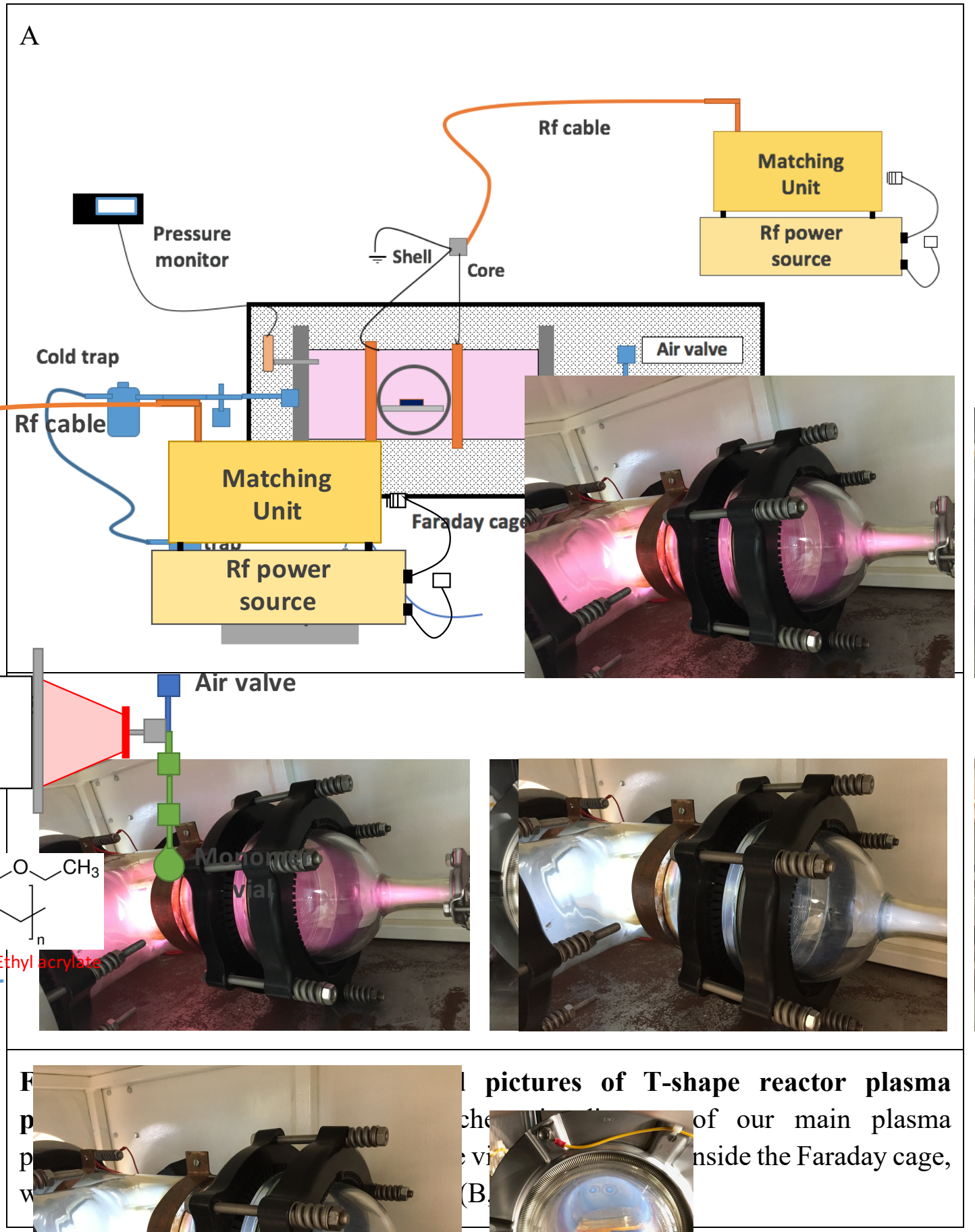

\subsubsection{Considerations for the operation with EA monomer}

Important design and operation considerations are highlighted here, as our experience tells us that they are critical to facilitate the polymerisation of monomers and of ethyl acrylate in particular.

Summary of the operation protocol. After testing and calibration of our equipment, and an understanding of its sensitivity to variation in its working parameters, we developed a robust plasma polymerization protocol to produce repeatable samples for this work, and in general in a standardised manner for other projects in our lab. 
A coating experiment always started with a 5 minutes stage of air plasma at around 0.1 mbar and 100W (50W for PCL substrates) of continuous power to clean and activate surfaces. Then a second stage of plasma polymerization is run at the planned power with the monomer vial valves used as a throttle to control the chamber pressure while the plasma is started and maintained. The detailed full protocol is included in appendices.

Chemical safety. The EA monomer can quickly swell and damage most rubber seals of common valves, connections and the pump if it's not properly protected. Custom made glass vials with Teflon stopper and Viton rings were commissioned in our school workshop. Only speedy valves with a fluoroelastomer diaphragm were used in the inlet part of the chamber. A cold trap, filled with liquid nitrogen during experiments, and a foreline trap, filled with ceramic absorbent pellets, were installed in the vacuum line to avoid monomer reaching the pump.

Control of EA pressure. We discuss here matters of practical control of the flow of EA monomer into the reactor that we found to be critical for its successful operation. EA is not very volatile compared to other monomers. EA's vapour pressure is rather low at room temperature, $28.8 \mathrm{mmHg}$ at $20^{\circ} \mathrm{C}$ [42] compared to $135 \mathrm{mmHg}$ for hexane [43] or $198 \mathrm{mmHg}$ for allylamine [44], both referenced at $20^{\circ} \mathrm{C}$. These and other monomers are frequently used in the literature in plasma polymerised coatings for biomedical applications 4. Our experiment trials followed a design of the monomer inlet valves and protocol of operation previously used successfully to operate with these other commonly used monomers. When building and testing our installations we found that using EA instead of hexane for instance makes it much more difficult to maintain a stable flow of monomer vapour and a stable pressure inside the reactor, at the required vacuum levels to spark an EA monomer plasma, around 0.2 mbar or lower. Initially we started using needle valves for the monomer inlet, as they are normally used in installations with the same overall design that operate with other monomers. We found that the needle valves clog and are damaged quickly with EA condensate when they are not fully open and even if fully open. This is a serious practical issue for the operation of the vacuum system, as unexpected pressure drops and the constant manual adjustment of the valves makes it very difficult to maintain a stable EA monomer plasma. It is also an important risk for the pump even with a cold trap set up before the pump inlet, as with these valves there can be bursts of monomer intake when a valve unclogs or it's opened too quickly. The EA monomer reaching the pump could easily damage its plastic components and contaminate the oil, changing its properties. In our system we installed a speedy valve for the monomer intake and a pump with better performance to have a better control of the pressure inside the reactor. We also developed an operation protocol to minimize condensation in valves working with the partial opening of two valves in the section of the monomer inlet to the plasma chamber: in the design of the installation used for the experiments in this study, we worked with a partial opening of the Teflon stopper in the monomer vial and of a speedy valve before the reactor to adjust and control the flow of monomer to the required level of vacuum needed to spark and stabilize a plasma reaction. 


\subsubsection{Working range of plasma reactor}

The working pressure for both plasma stages (air and monomer) was adjusted in a systematic manner for all experiments. The optimal working pressure was prestablished for our experiments as the highest pressure possible to maintain a stable plasma while reflected power was minimum or near minimum. This pressure depends on the applied RF power, on the physical and electrical characteristics of the specific reactor, and also on the nature of the plasma phase in the chamber (air, oxygen, monomer, etc.) [45]. After assembling our custom-made plasma reactor, we determined the range of working pressures and powers. For every commercial or custom-made plasma reactor, there is a range of pressures and powers where plasma glow discharge is possible, depending on the gas or monomer present inside the reactor. When the pressure is too high the plasma doesn't spark, or if the plasma is running and the pressure rises the reflected power will go progressively up until, above a critical limit, the plasma shuts off (Figure 4).

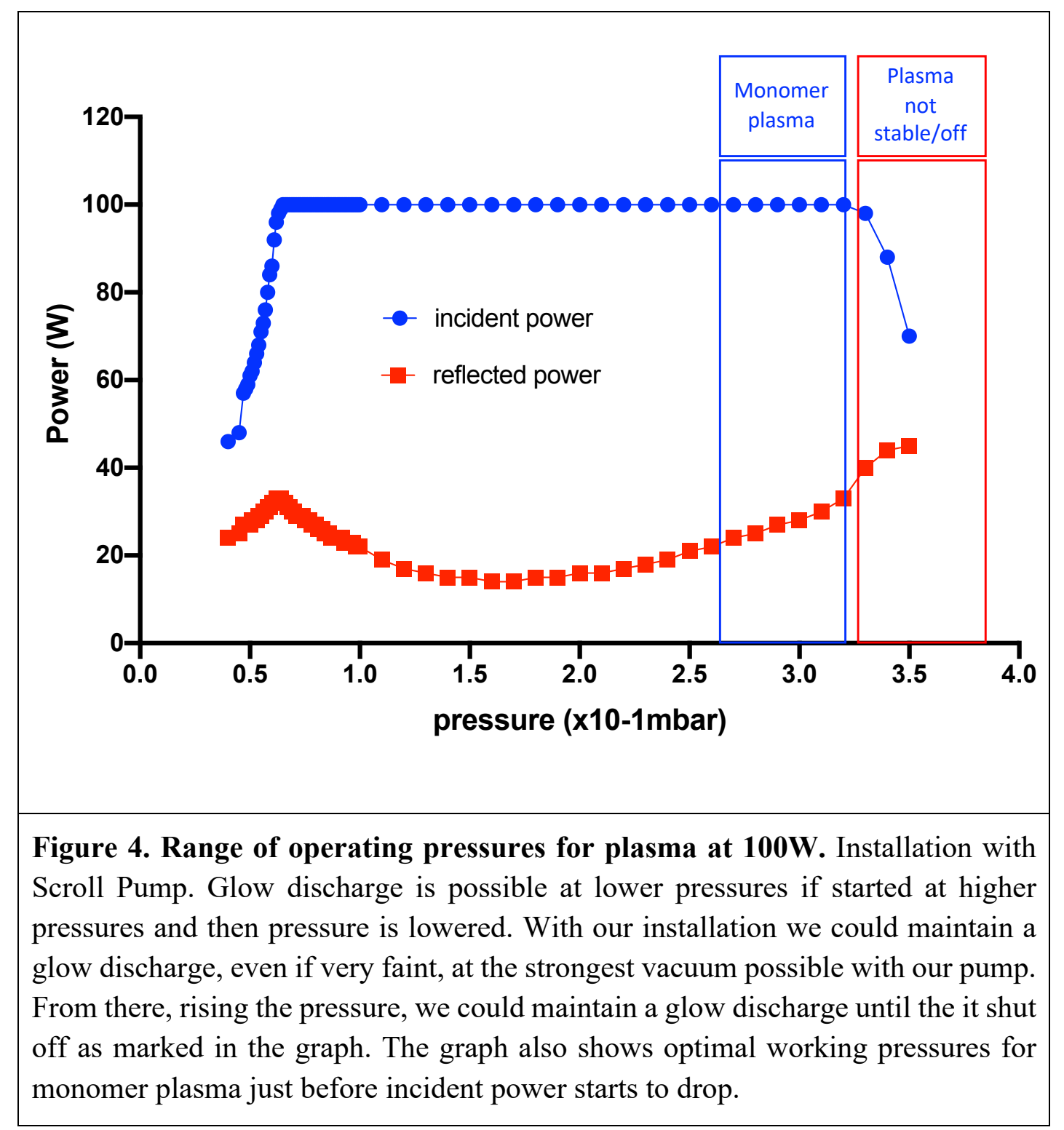


The optimal working pressure was chosen for our experiments as high as possible to maintain a glow discharge plasma while reflected power was minimum or near minimum. This maximises the density in the plasma volume and the deposition efficiency. This optimal range of pressures for polymerisation depends on the applied RF power, on the physical and electrical characteristics of the specific reactor, and also on the nature of the plasma phase in the chamber (air, oxygen, monomer, etc).

With our custom-made installation, we worked in the pressure ranges in Table 1 for the plasma polymerisation stage.

\begin{tabular}{|c|c|c|}
\hline Power $(\mathrm{W})$ & (A) Pressure (mbar) & (B) Pressure (mbar) \\
\hline 100 & $2.6-3.1 \times 10^{-1}$ & $5.5-6.2 \times 10^{-1}$ \\
\hline 75 & $1.9-2.7 \times 10^{-1}$ & $4.5-4.9 \times 10^{-1}$ \\
\hline 50 & $1.6-2.3 \times 10^{-1}$ & $3.5-3.8 \times 10^{-1}$ \\
\hline 25 & $1.2-1.5 \times 10^{-1}$ & $2.5-2.6 \times 10^{-1}$ \\
\hline 15 & $1.0-1.3 \times 10^{-1}$ & $1.8-2.2 \times 10^{-1}$ \\
\hline
\end{tabular}

Table 1. Range of optimal working monomer pressures during the plasma polymerisation stage. Column (A) shows optimal pressures for the installation with a scroll pump, at five Rf powers. Column (B) for installation with a less powerful oil rotary pump.

\subsubsection{Other built and tested reactor designs}

Before settling on the design described in section 1.1 for our main plasma reactor, we built and tested several prototypes. This was a very useful first stage of our work-plan, to check how the different designs and available equipment components responded to our experimental requirements in real lab operation, and in the process to learn how to modify and adapt the standard plasma chamber designs to better perform to our requirements: (i) we needed to produce coated flat and 3D samples (scaffolds, porous granules, tubes) efficiently, with quick and easy access to samples and general ease of use, to produce samples in sufficient numbers to supply not only our characterisation efforts but also other parallel projects planning to use a successful functional performance of plasma EA coated surfaces in vitro and in vivo; (ii) the reactor needed to accommodate and homogeneously coat batches of samples where each sample is of at least several centimetres in their main dimension; (iii) particular safety concerns 
had to be prioritised to minimise the risk of accidents or simply disturbing other works in a shared lab when handling the EA monomer. For the design and construction of the plasma reactors we followed known working designs of lab plasma chambers used to polymerise other monomers as discussed in the introductory sections.

\subsubsection{Cylindrical chamber with inner plate electrodes}

Our first two fully built prototypes were based in a capacitively coupled plasma design with a parallel plate configuration of the electrodes inside the vacuum chamber. We used a glass cylindrical chamber with a volume of around 20L for a first prototype (Figure 4) and a similarly shaped chamber, but lighter, with a much smaller volume of around 1L, for a second one (Figure 6), with overall better behaviour on handling and vacuum setup.

Electrical grounding of the installation. During the assembly and tests of our first prototypes we struggled to spark a plasma with the available electrical connections that failed to properly connect the Rf cable to the electrodes in the system, in particular the grounding to electrode cable. Having a good grounding and a clean split of the shell of the Rf cable into two grounding connections, has proved to be a very important practical matter for the setup of custom-made plasma reactors and we have detailed our experience in this matter here in this section.

Our very first reactor setup was used a radio frequency power supply (Nordiko Limited) that works at $13.56 \mathrm{MHz}$ up to $2,000 \mathrm{~W}$. This Rf source and matching unit were commissioned from an old but working plasma etching chamber. When setting up this installation with them we struggled to spark a stable plasma in our chamber, with the Rf source breaking on connection no matter the level of vacuum.

We speculated and checked potential causes for this: (i) We thought the upper electrode might be too small and/or its shape reflected all the energy to the matching unit, and we tried a different design of the upper electrode with more mass and several different shapes (Figure 7); (ii) as these were old pieces of equipment we thought there might be a shortcut in the installation, with our first suspect being a shortcut in the source or matching unit, through a broken capacitor.

We changed the Rf source to a newer one (Coaxial Power System Ltd.) that works at 13.56 MHz up to 150Watt. When disconnecting the old Rf source and setting up the new one we realised the grounding cable, connecting the shell of the Rf cable to ground was poorly connected to one of the electrodes in our installation. 


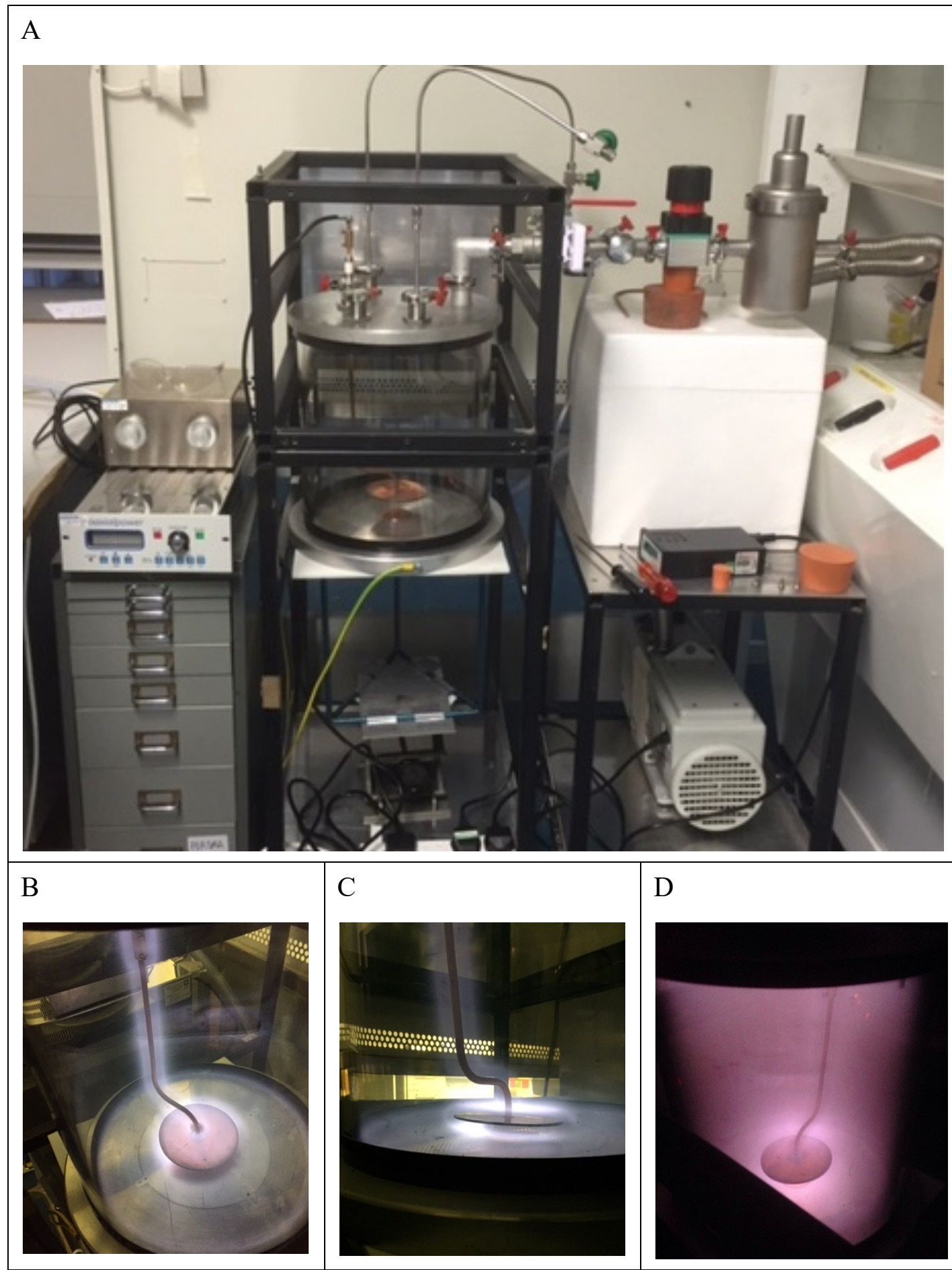

Figure 5. Pictures of first cylindrical chamber plasma reactor prototype. All pictures show the cylindrical chamber (20L) with inner plate electrodes. (A) Whole installation with pump, rf source, matchin unit, monomer inlet and cold trap visible. (B) and (C) show a glow discharge with air plasma with bad electrical grounding, and (D) with a better grounding.

Ease of use of the installation. The first reactor built, a 20L glass cylindrical chamber, shown in Figure 5, had several practical shortcomings. The top and bottom steel plates were heavy and difficult to move to get samples in and out of the chamber 
for experiments. We set up a platform with a screw wheel to move the bottom plate down more safely and quicker but opening and closing down the chamber still took over ten minutes each time and made the whole operation very cumbersome.

With components from the school workshop we built a second prototype based in the same design, with a much lighter chamber, and a volume of around 1L (Figure 6). with overall better behaviour on handling and vacuum setup. It was easier to perform experiments with this prototype, quicker to handle the samples in and out of the reactor and to make vacuum.

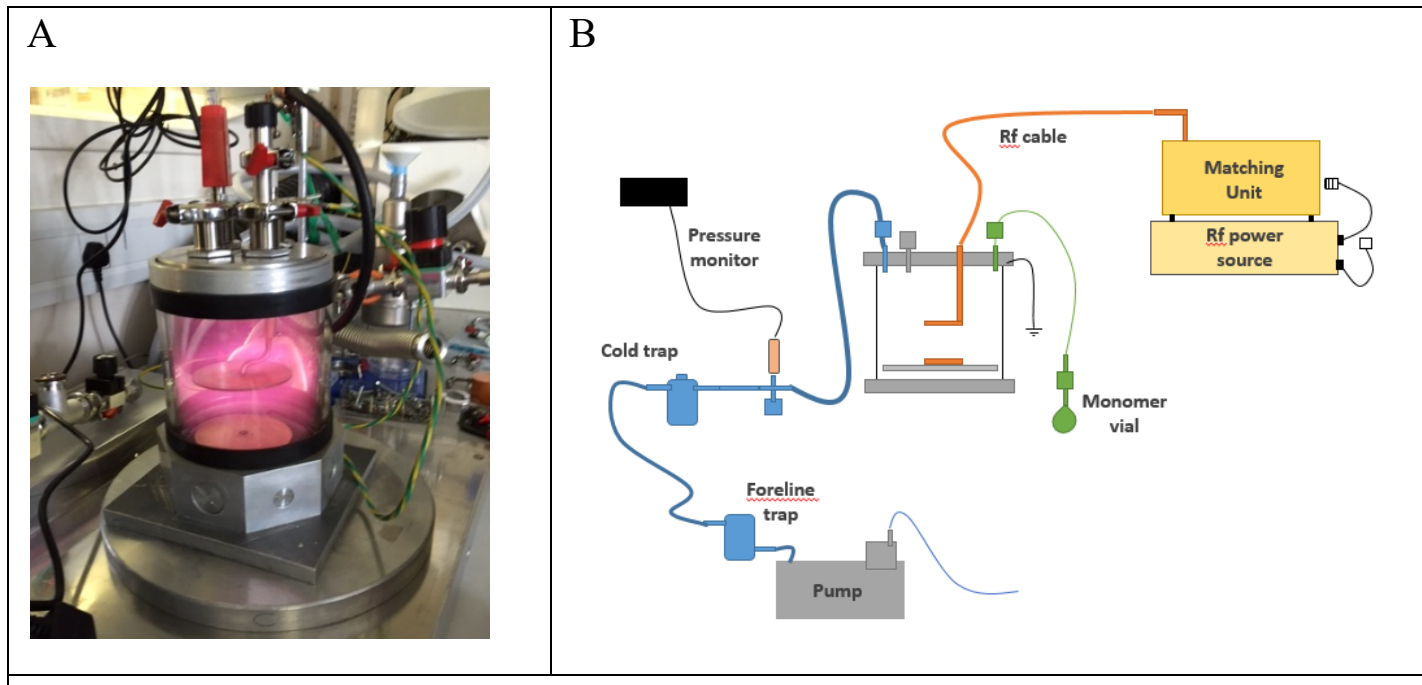

Figure 6. Plasma reactor prototype with a low volume cylindrical chamber. (A) Picture of the prototype working with an air plasma glow discharge. The cylindrical chamber has a volume of 1L, with inner plate electrodes. (B) Schematic diagram of the whole plasma polymerisation installation, showing the $\mathrm{Rf}$ source and other parts of the system.

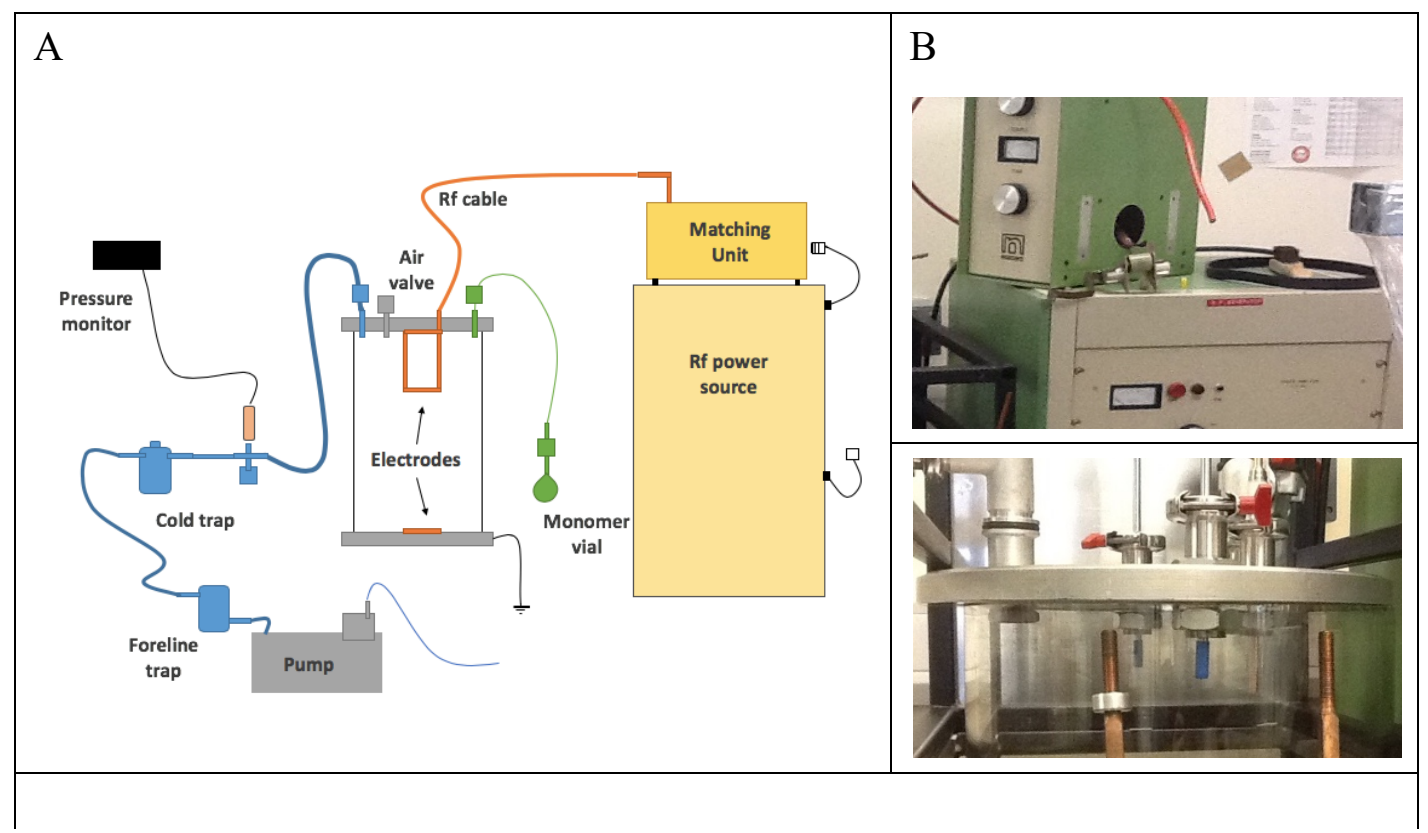




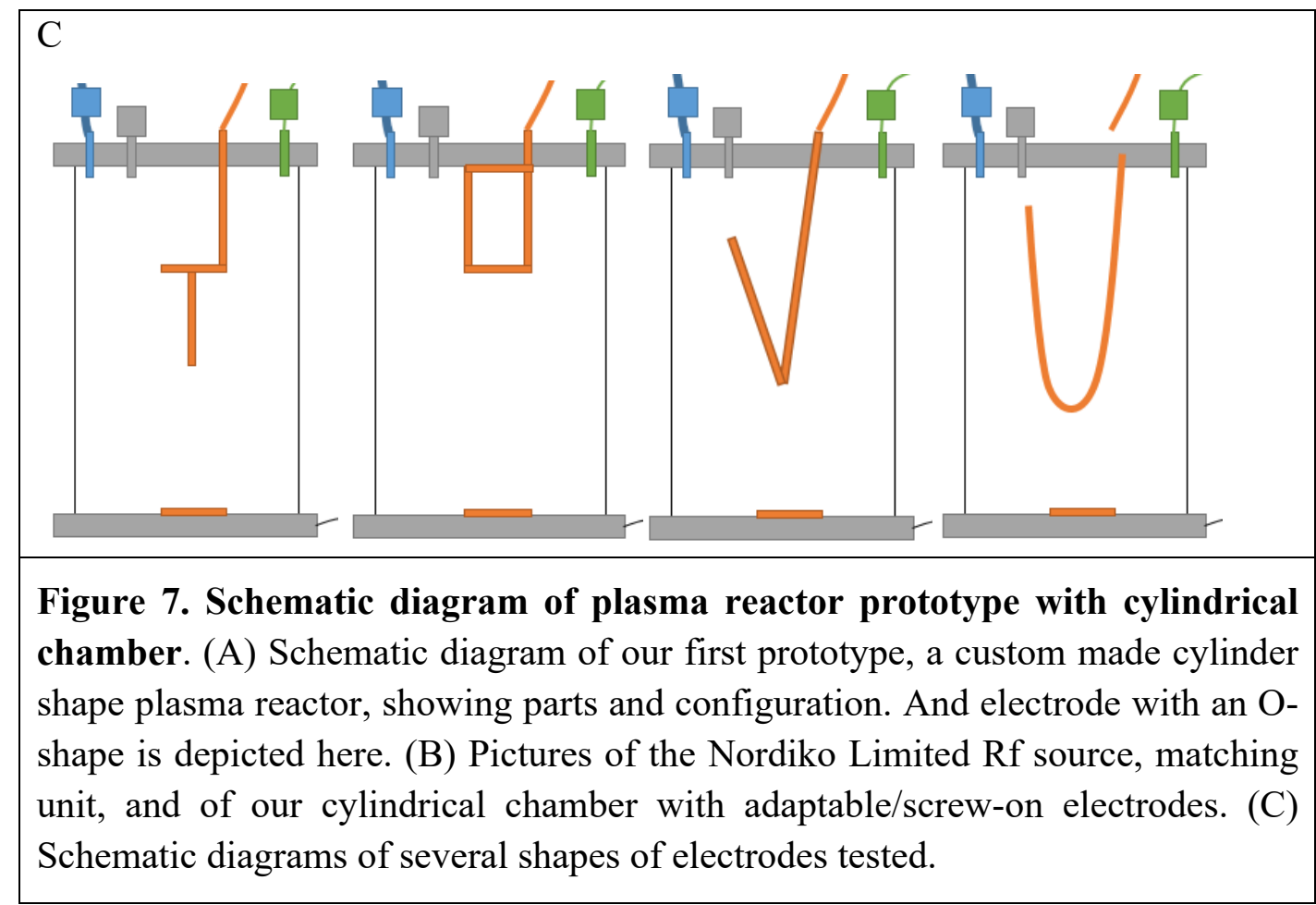

\subsection{Plasma polymerised materials}

Using the equipment setup described in section 4.1.1 we produced plasma polymerised coatings on a variety of substrates, including glass flat surfaces, spin coated polymers, porous scaffolds, and surfaces with functional microtopographies of two different kinds. We have characterised the materials produced under different experimental conditions, assessing the effect of applied radiofrequency (Rf) power, duration of plasma deposition, and underlying substrate roughness, on the polymerised coating properties.

Once protocols and standard coating properties were stablished, we used this equipment to coat several other substrates that are not described in detail here, and that are being used in ongoing separate studies by other researchers in the team, i.e. osteogenic PEEK surfaces, hollow tubes for a radial bone graft in a small animal model, synthetic scaffolds, beads and microparticles, and allogenic canine bone graft chips for an in vivo veterinary trial [7]. The latter, with a successful intervention on a dog, as a first veterinary patient treated with this bone regeneration technology, received a lot of general media attention [46]. The trial is still currently running at the Small Animal Hospital at the University of Glasgow.

\subsubsection{Deposition rate, thickness of coatings}

The plasma polymerization conditions used in this work were controlled by three parameters: power applied to the plasma chamber, plasma treatment time, and pressure during the plasma polymerisation stage. 
The deposition rate of the plasma polymerised materials on the sample surfaces has been characterized by measuring the thickness of coatings deposited at various experimental conditions. We used AFM to measure the depth of a clean scratch performed on the surface of a sample after a deposition experiment (Figure 8). The scratch was made carefully by running a razor along the surface of a coated sample as to scratch the polymerised material on the surface down to the underlying glass.

By changing the parameters of the plasma deposition experiments, these scratch tests have showed that the deposition rate remains fairly constant during the monomer plasma polymerisation stage for a given experimental condition (table 2). After the first seconds of experiment the rate of coating in our installation was from around 6.5 $\mathrm{nm}$ per minute to over $10 \mathrm{~nm}$ per minute. We were able to produce nano scale coatings onto glass ranging from $34 \pm 7 \mathrm{~nm}$ to $309 \pm 28 \mathrm{~nm}$ for depositions at $4.5 \mathrm{~kJ}$ and $180 \mathrm{~kJ}$ respectively (Table 2). Thickness of the coatings slightly increased with increasing power. The relationship between the applied energy and the obtained PEA coating seems to follow a hyperbolic curve model (Figure 9A inset). As a reference, spin coated PEA(SC-PEA) produced coatings of a thickness well over one micron.

For a given power, pressure during the plasma polymerisation stage has a more critical effect on the rate of coating (Figure 9B). We changed the working pressure for the plasma polymerisation stage by changing the vacuum pump (scroll or oil pump). We maintained the same operating protocol with both pumps so that the monomer plasma polymerisation stage was run at maximum incident power and close to minimum reflected power, at different levels of vacuum depending on the pump used.

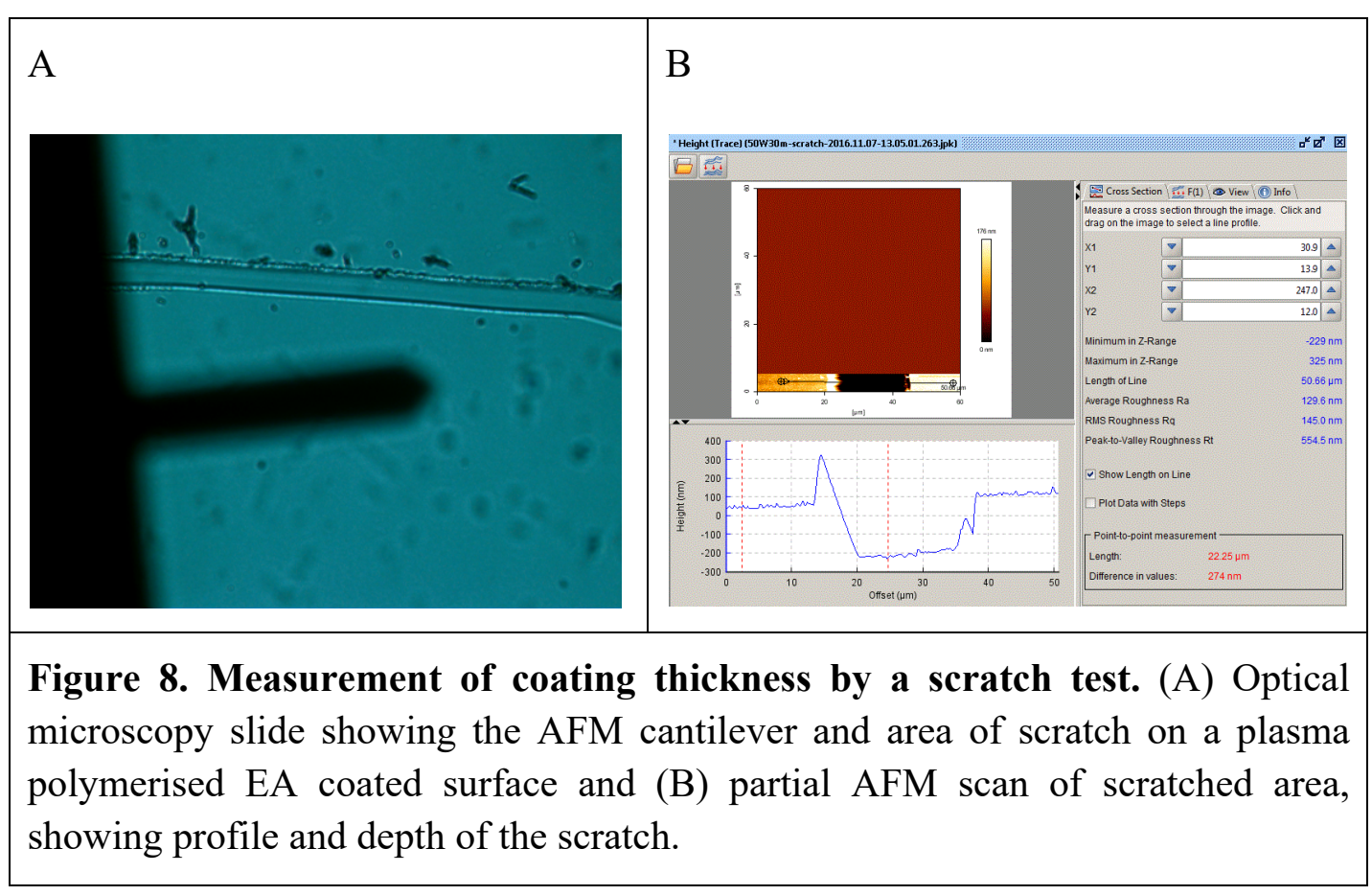




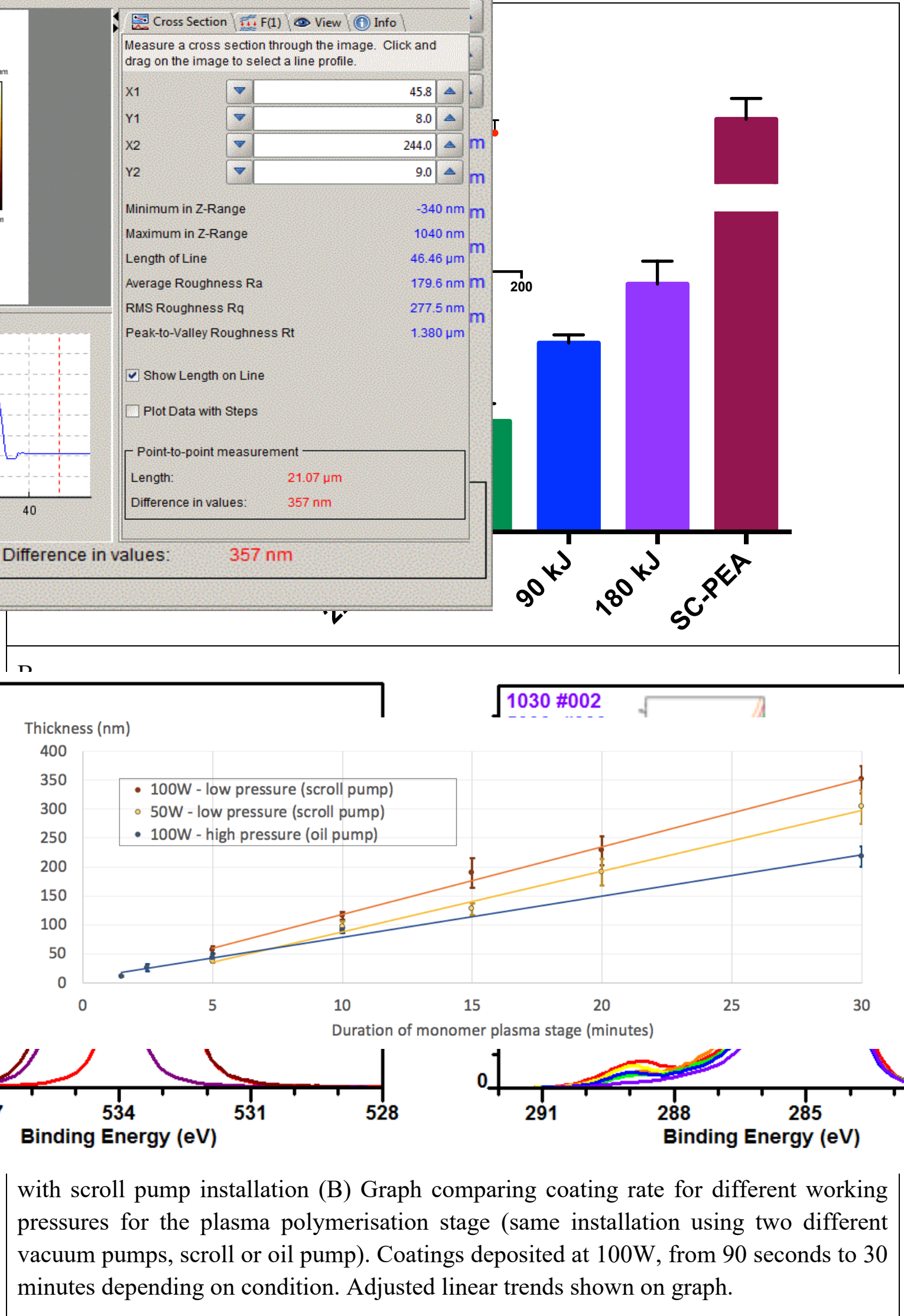




\begin{tabular}{|l|c|c|c|}
\hline $\begin{array}{c}\text { Sample } \\
\text { condition }\end{array}$ & $\begin{array}{c}\text { Energy } \\
(\mathbf{k J})\end{array}$ & $\begin{array}{c}\text { Thickness } \\
\mathbf{( n m})\end{array}$ & $\begin{array}{c}\text { Coating } \\
\text { rate }\end{array}$ \\
\hline 15 W 5 mins & 4.5 & $34 \pm 7$ & $6.8 \pm 1.4$ \\
\hline 50 W 5 mins & 15 & $43 \pm 7$ & $8.6 \pm 1.4$ \\
\hline 25 W 15 mins & 22.5 & $118 \pm 14$ & $7.9 \pm 0.9$ \\
\hline 50 W 15 mins & 45 & $138 \pm 22$ & $9.2 \pm 1.5$ \\
\hline 50 W 30 mins & 90 & $236 \pm 10$ & $7.9 \pm 0.3$ \\
\hline 100 W 30 mins & 180 & $309 \pm 28$ & $10.3 \pm 0.9$ \\
\hline Spin coating & - & $1272 \pm 117$ & - \\
\hline
\end{tabular}

Table 2: Thickness measurements. AFM scratch test results for coating conditions tested listing coating rates. All coatings deposited with scroll pump installation. Results listed as Average \pm SD.

First seconds and minutes of deposition. In our thickness measurement experiments, performing an AFM scratch test on coated samples, we usually produced and measured reproducibly samples after five minutes of monomer plasma deposition. However, we also ran a series of deposition experiments for very short deposition times, with the first plasma reactor prototype, using an oil pump. The AFM scratch test technique is at the limit of its practical use to measure coating at conditions under 90 seconds. The installation with the oil pump produces a higher working pressure for the plasma glow discharge and a lower coating rate.

In these series of experiments, we were able to show that, for the first seconds and minutes of deposition, the rate of coating maintains a linear trend consistent with the rates for longer deposition times, around $9 \mathrm{~nm}$ per minute for the fully tested condition.

Results are showed in Figure 10.

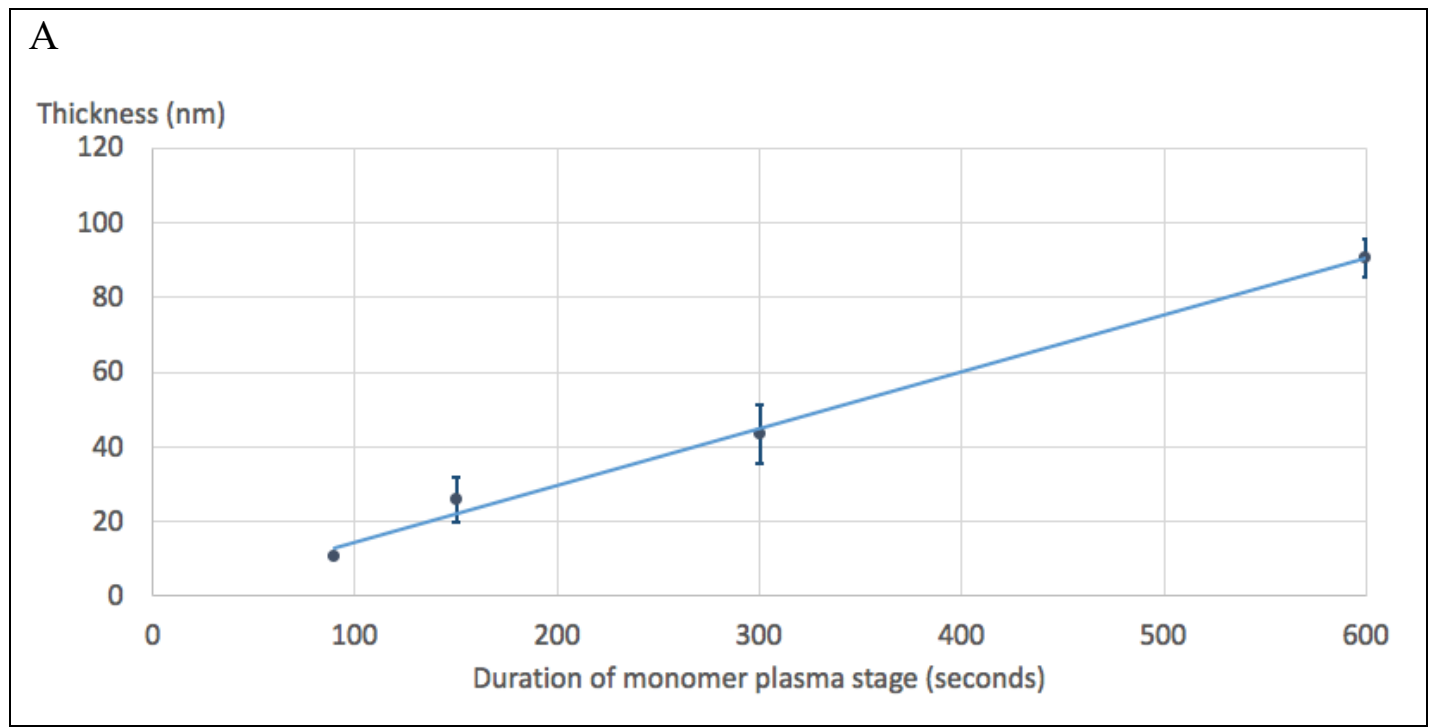



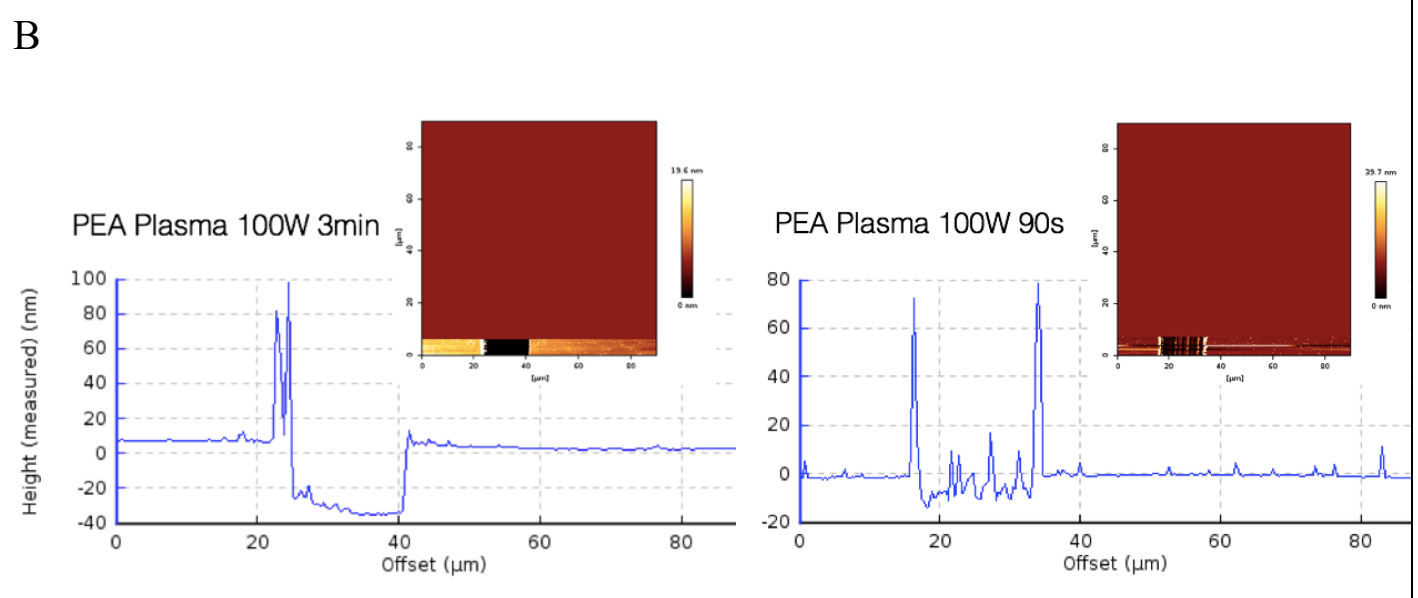

Figure 10. Plasma polymerized coatings. Thickness measurements. Short deposition times. (A) Graph showing coating rate for short deposition times (installation using oil pump). Coatings deposited at $100 \mathrm{~W}$, from 90 seconds to 10 minutes. Adjusted linear trend shown on graph. (B) Example of AFM profiles of scratches at very short deposition times, i.e. 3 minutes (left) and 90 seconds (right), at the limit of the practical use of this technique to measure coating thickness.

\subsubsection{Deposition rate in different areas of the chamber}

The consistency of deposition rate at different points within the chamber was assessed in a similar manner by placing coverslips on twenty-two different positions throughout the chamber including ten onto the sample holding shelf in the middle of the chamber. We found moderate variations on deposition rate (Figure 11) along the chamber, with average values from around 5 to over $10 \mathrm{~nm}$ per minute, with plasma depositions at $90 \mathrm{~kJ}$, with the exception of the area under the front (outlet) electrode that produced much faster deposition rates.

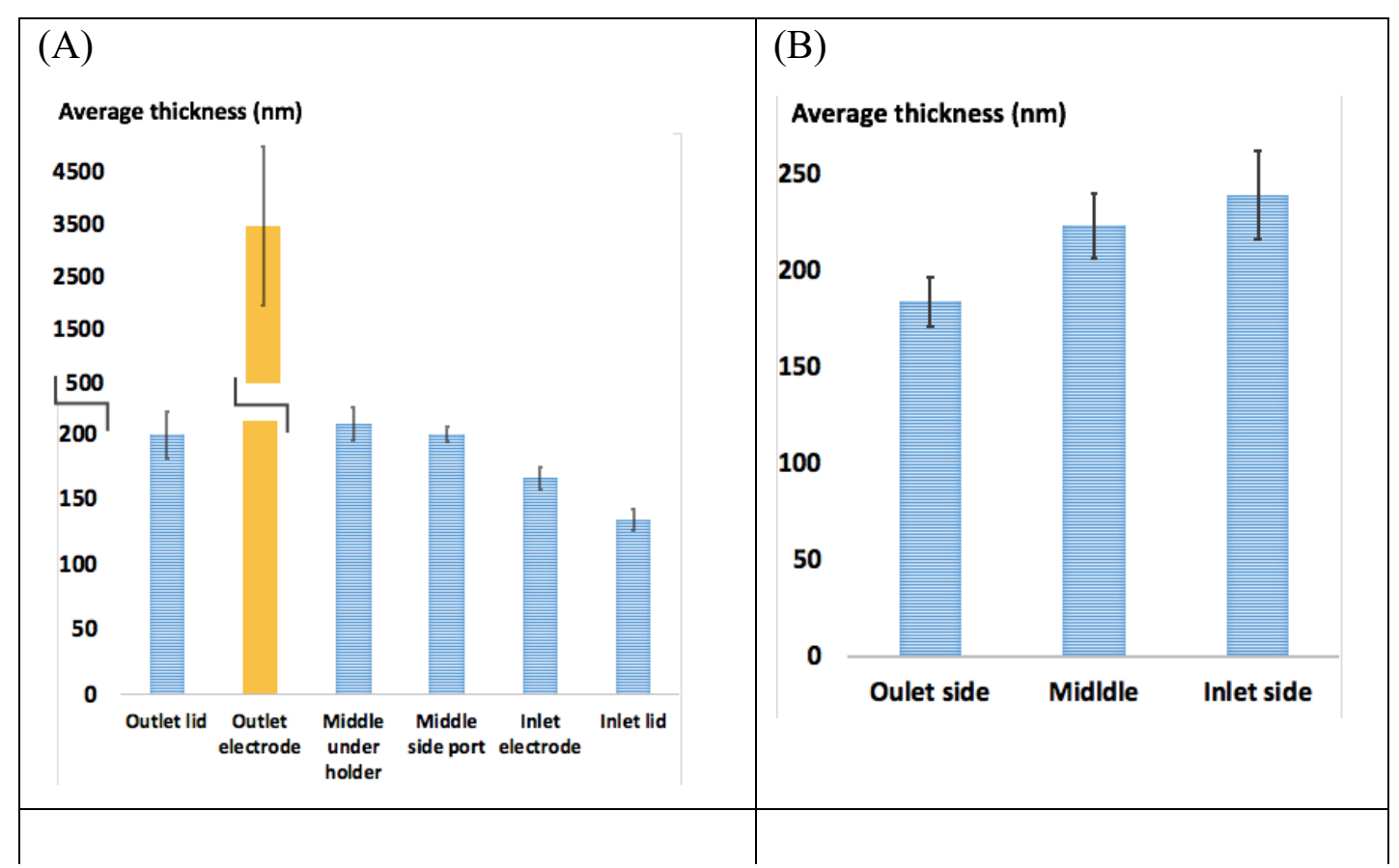




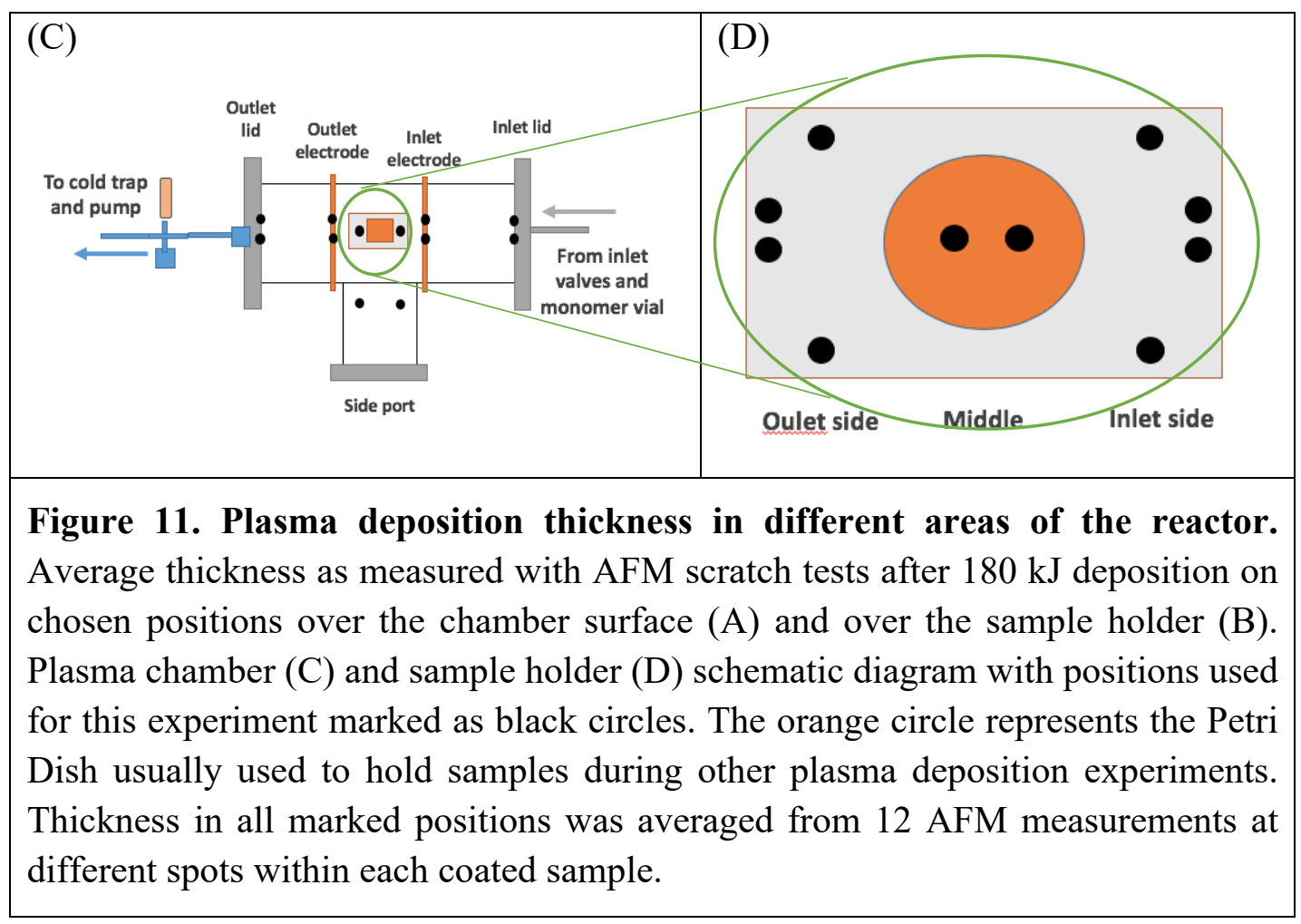

\subsubsection{Surface of polymerised coatings}

We used Atomic Force Microscopy (AFM) to look at the morphology of the polymerised surfaces at the sub-micrometric scale and to verify any variation of surface morphology with plasma Rf power and duration of experiment.

Samples were coated with plasma polymerised EA on experiments at different powers and duration times. We have shown that these parameters control the coating thickness with a small but noteworthy effect on the coating rate. The coating rate with our preferred installation set-up and working plasma pressure was usually from 8 to 10 $\mathrm{nm} / \mathrm{min}$ as detailed in section 1.1.4. Samples were produced from up to $100 \mathrm{~W}$ to $15 \mathrm{~W}$, with durations of 30 minutes to 5 minutes for the plasma polymerisation stage. Control surfaces are shown on Figure 10 and a selection of AFM scans a full study of surfaces at different plasma polymerisation conditions for the $50 \mathrm{~W}$ and $100 \mathrm{~W}$ experiments are presented in Figure 13 and Figure 14 respectively.

Figure 15 summarises this part of our work, showing side by side AFM scans of flat (12mm microscopy glass coverslips or microscopy slides) surfaces just after the initial air plasma stage, after 5 minutes and after 30 minutes of plasma EA monomer deposition.

\subsubsection{Roughness of coating on flat substrates}

With these experiments, in the case of a flat underlying substrate, we also confirmed that the polymerised material coats the surface homogeneously, and that the roughness of the coating remains very low, just slightly above the 'flatness' of the underlying 
glass or spin coated polymer surfaces (Figure 16), and within the same Average Roughness $\mathrm{Rq}$ of around $0.6 \mathrm{~nm}$ no matter the duration of the deposition or the thickness of the coating.

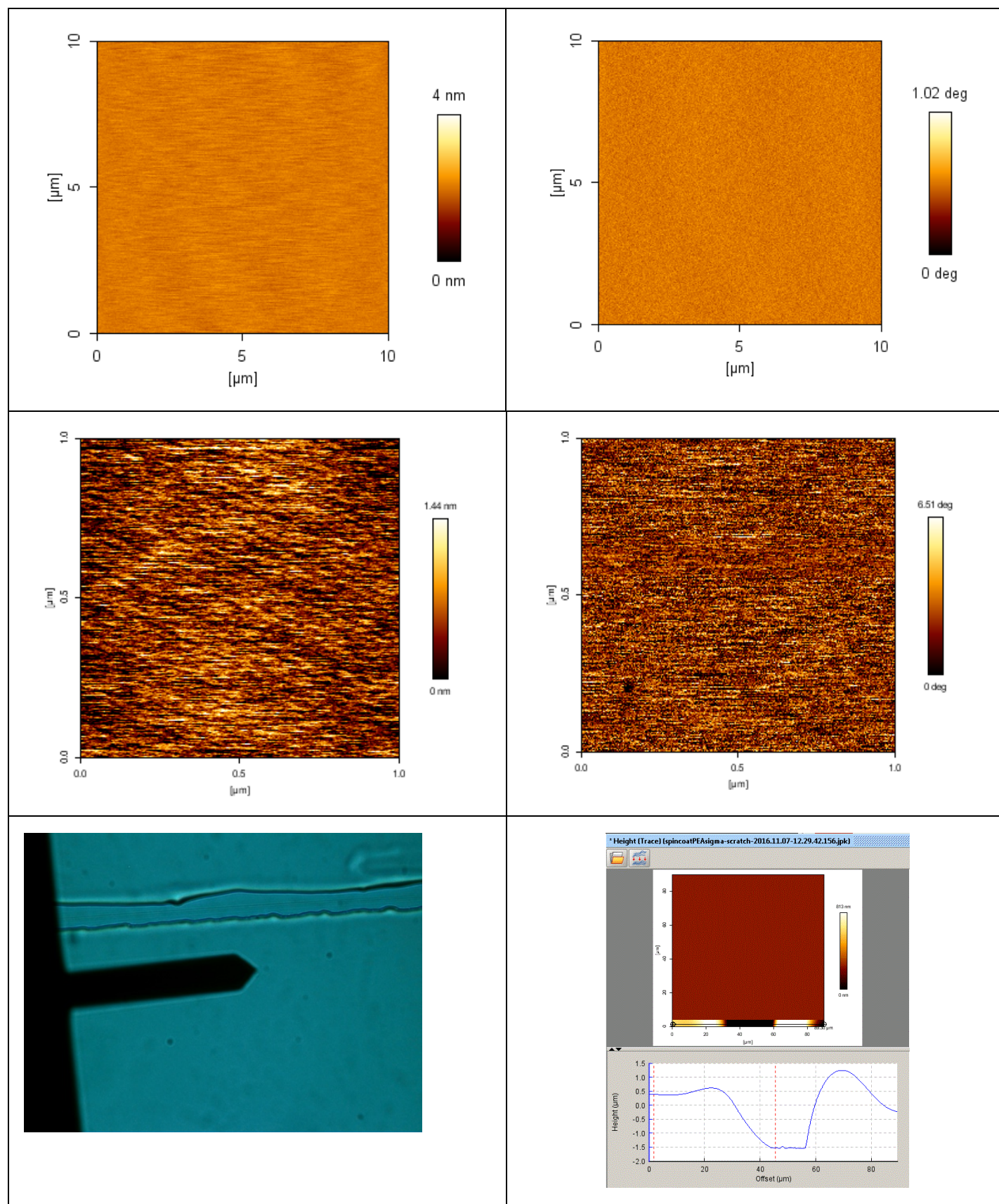

Figure 12. AFM scans of plasma polymerised surfaces. Control surfaces. AFM scans, height on left column and phase on the right, of (first row) glass surface after five minutes of air plasma at $100 \mathrm{~W}$ of incident $\mathrm{Rf}$ power. No scratch found in scratch test. On second row is shown a spin coated PEA (Sigma 19,4\% in Toluene) surface on a glass coverslip. Thickness average for the spin-coated control samples is $1.59 \mu \mathrm{m}(\sigma$ of 0,26$)$. One selected measurement shown in third row. 


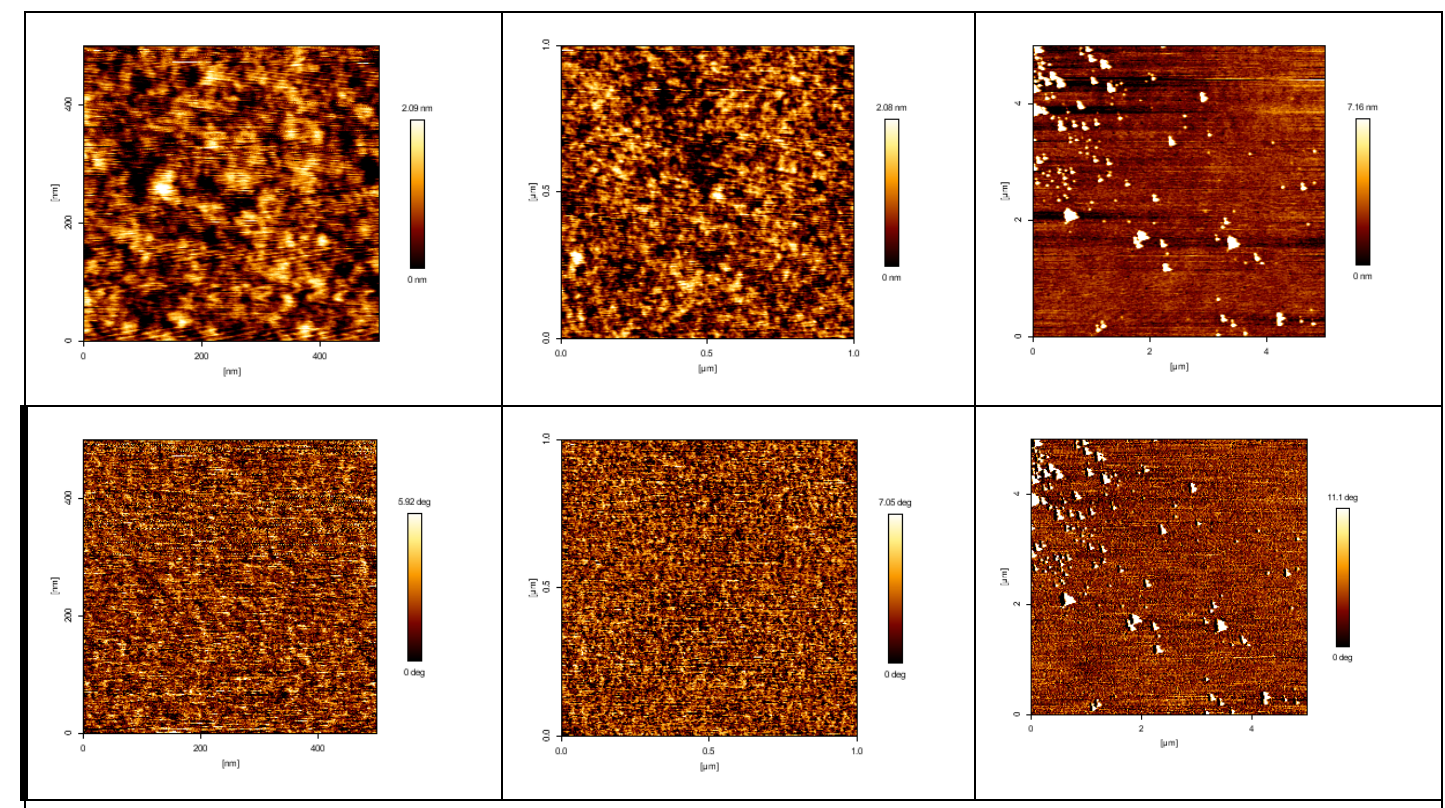

(A) 5 minutes at $50 \mathrm{~W}(15 \mathrm{~kJ})$

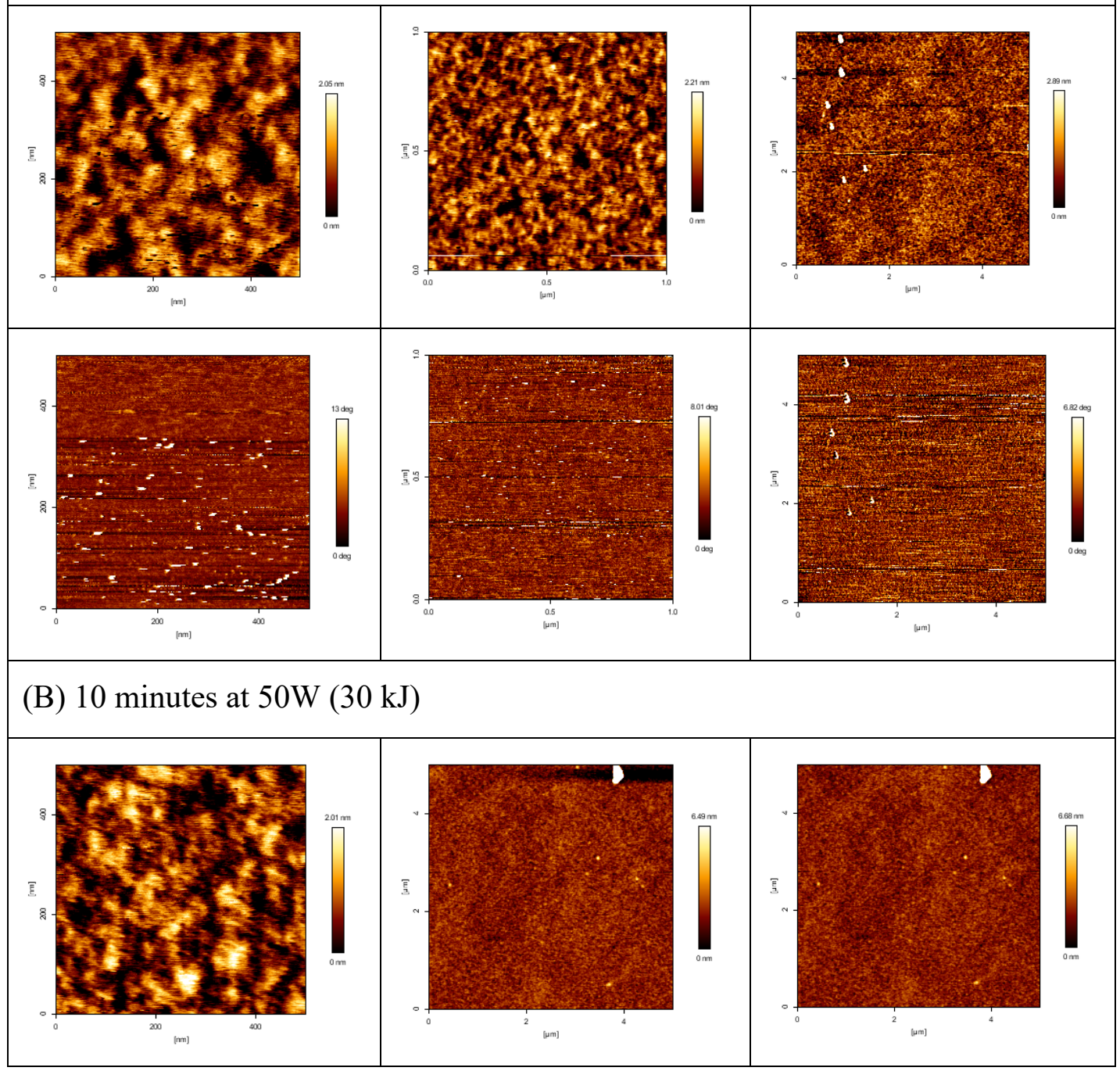




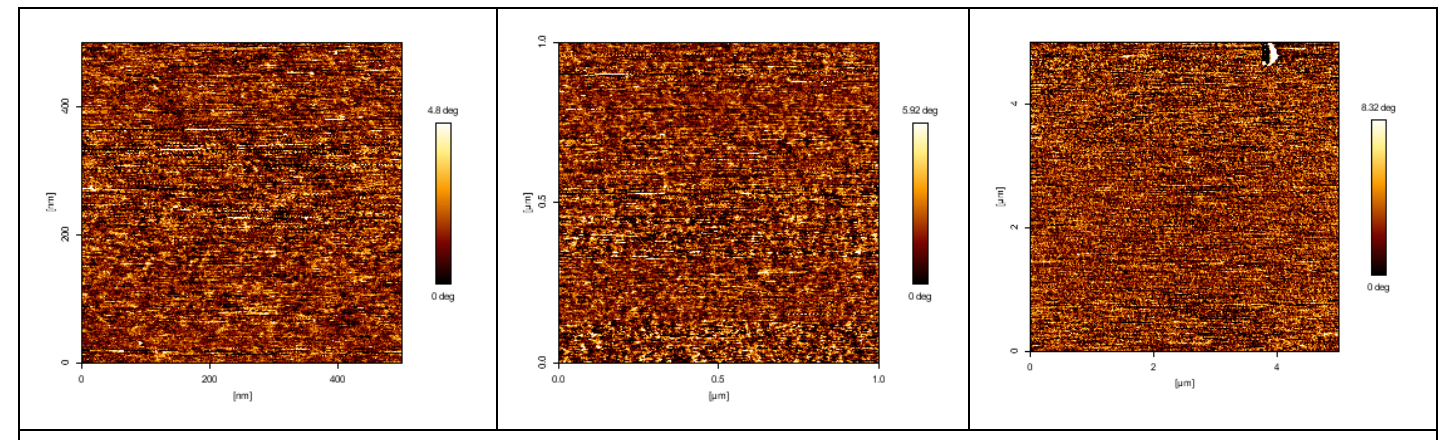

(C) 15 minutes at $50 \mathrm{~W}(45 \mathrm{~kJ})$

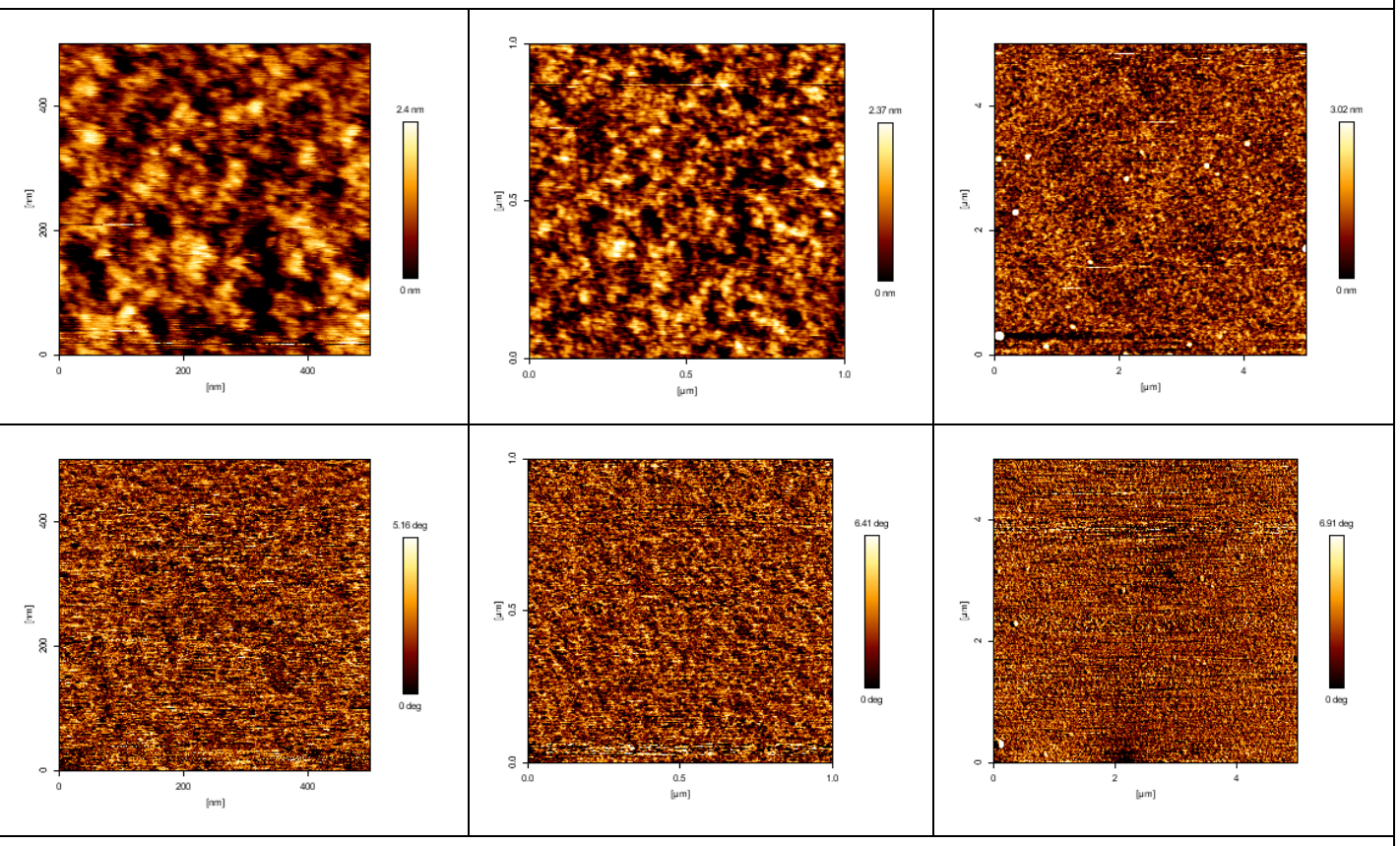

(D) 20 minutes at $50 \mathrm{~W}(60 \mathrm{~kJ})$

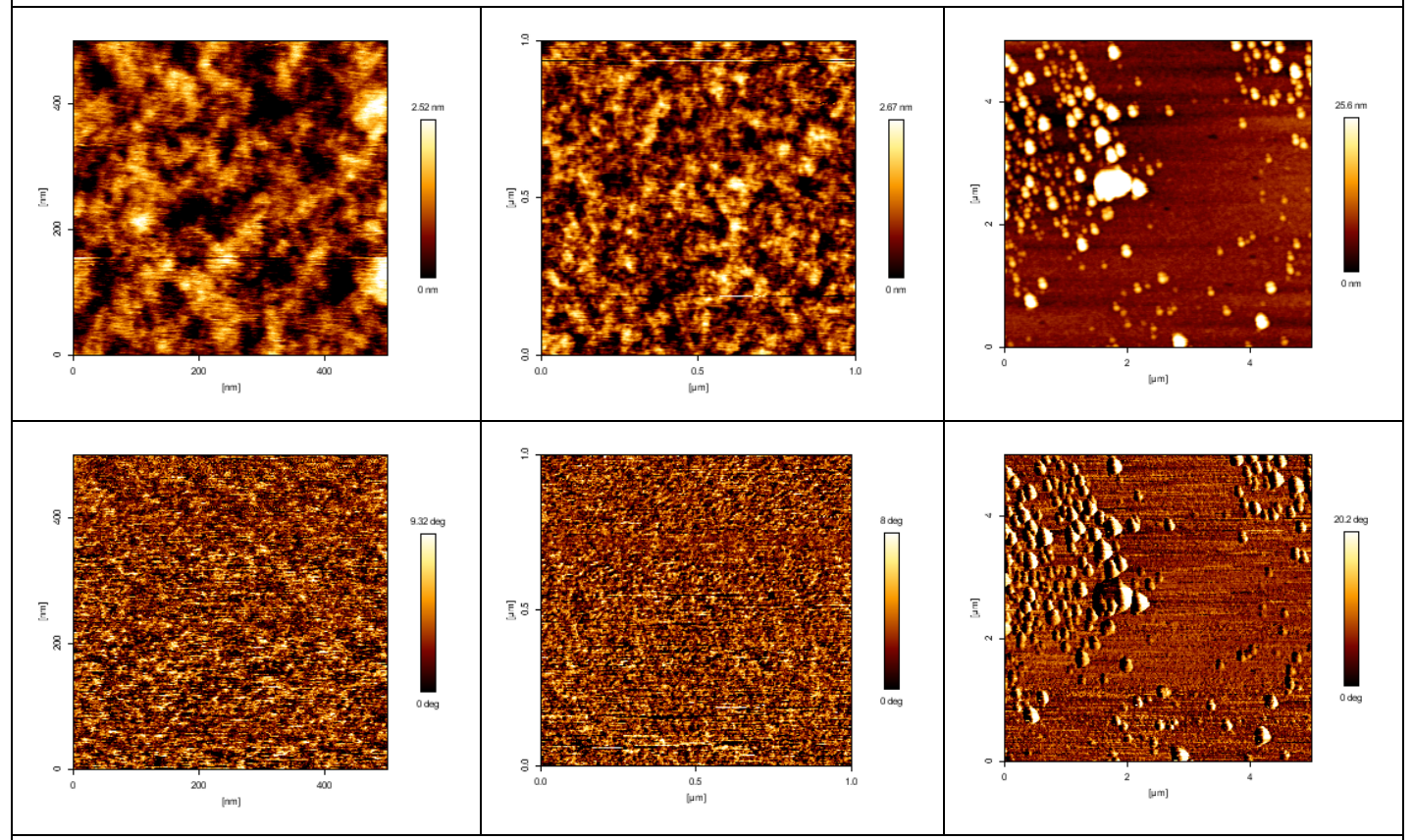

(E) 30 minutes at $50 \mathrm{~W}(75 \mathrm{~kJ})$ 
Figure 13. AFM scans of plasma polymerised surfaces, effect of duration of plasma. AFM scans of glass surfaces coated with plasma polymerised EA at $\mathbf{5 0} \mathbf{W}$ of incident Rf power, with a deposition duration/energy of A) 5 minutes $/ 15 \mathrm{~kJ}, \mathrm{~B}$ ) 10 minutes $/ 30 \mathrm{~kJ}, \mathrm{C}) 15$ minutes $/ 45 \mathrm{~kJ}, \mathrm{D}) 20$ minutes $/ 60 \mathrm{~kJ}$ and E) 30 minutes $/ 75$ $\mathrm{kJ}$. Scans are showed with height in the top row and phase in the bottom row for each experimental condition. Scans width are 500nm (left column), 1um (middle column) and 5um (right column).

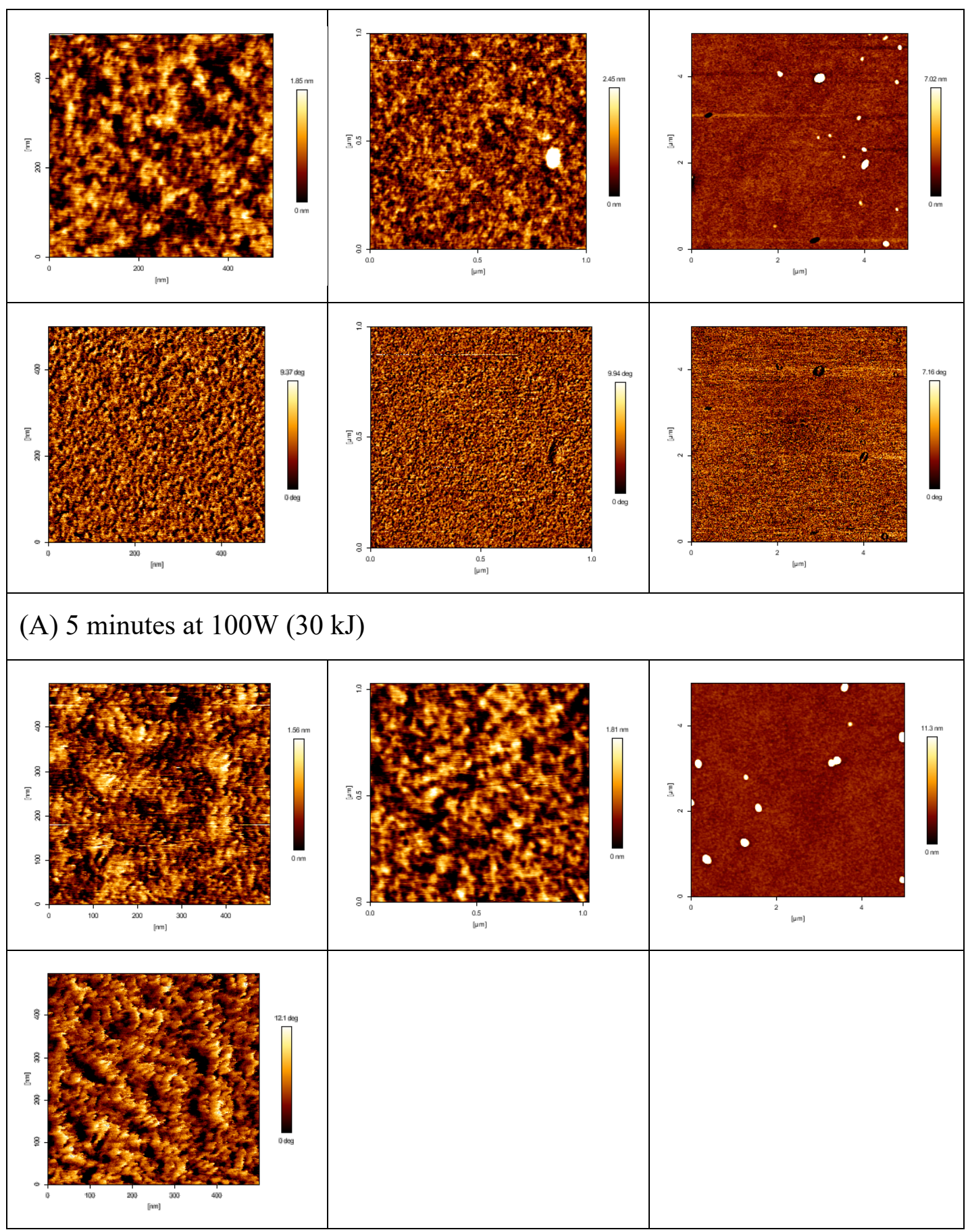


(B) 10 minutes at $100 \mathrm{~W}(60 \mathrm{~kJ})$
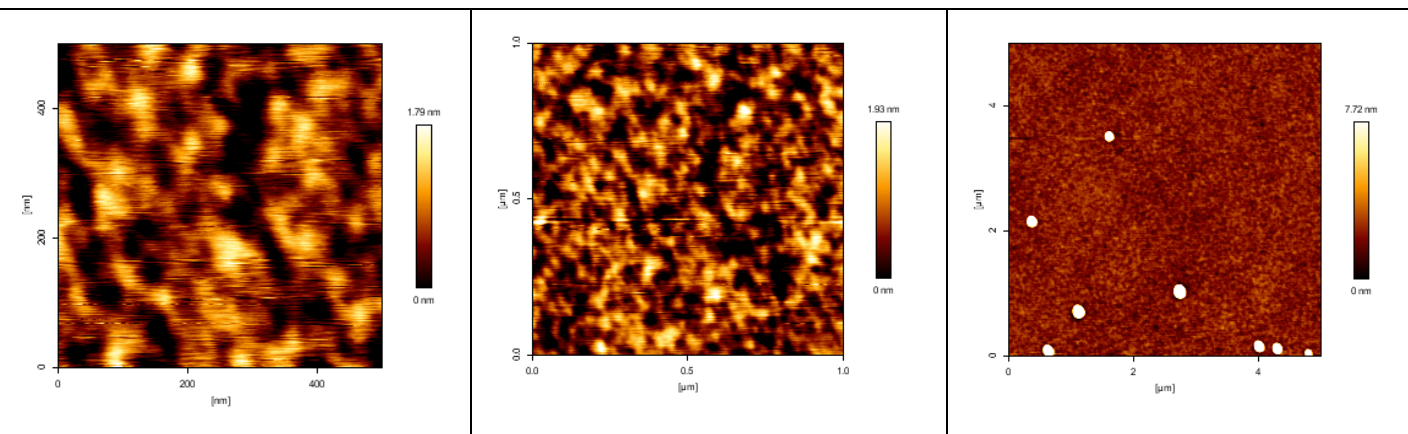

(C) 15 minutes at $100 \mathrm{~W}(90 \mathrm{~kJ})$

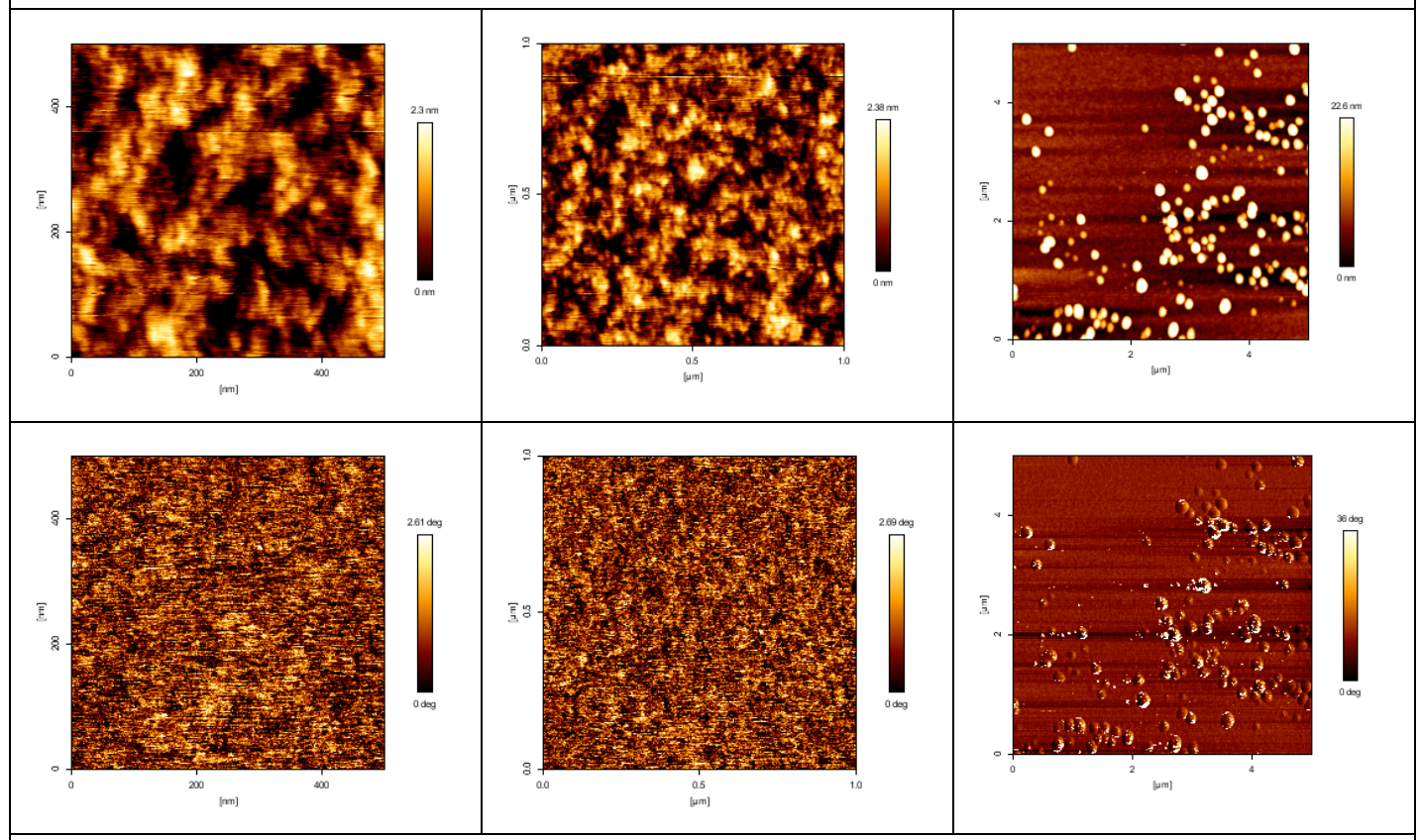

(D) 20 minutes at $100 \mathrm{~W}(120 \mathrm{~kJ})$

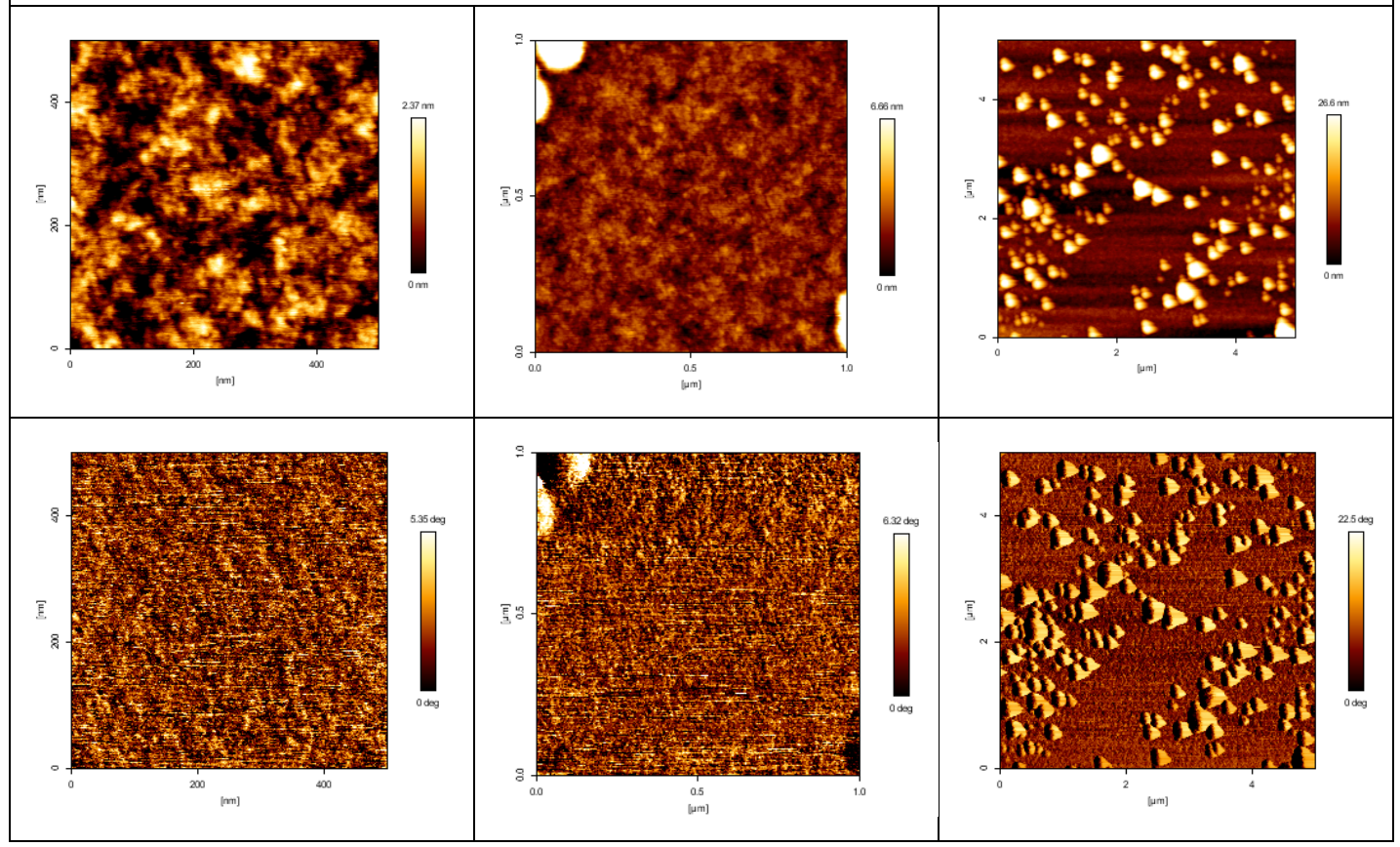


(E) 30 minutes at $100 \mathrm{~W}(180 \mathrm{~kJ})$

Figure 14. AFM scans of plasma polymerised surfaces, effect of duration of plasma. Scans of glass surfaces coated with plasma polymerised PEA at 100W of incident Rf power, with a deposition duration/energy of A) 5 minutes $/ 30 \mathrm{~kJ}, \mathrm{~B}) 10$ minutes $/ 60 \mathrm{~kJ}, \mathrm{C}$ ) 15 minutes $/ 90 \mathrm{~kJ}, \mathrm{D}) 20$ minutes $/ 120 \mathrm{~kJ}$ and E) 30 minutes $/ 180$ $\mathrm{kJ}$. Scans are showed with height in the top row and phase in the bottom row for each experimental condition. Scan width are 500nm (left column), 1um (middle column) and 5um (right column).

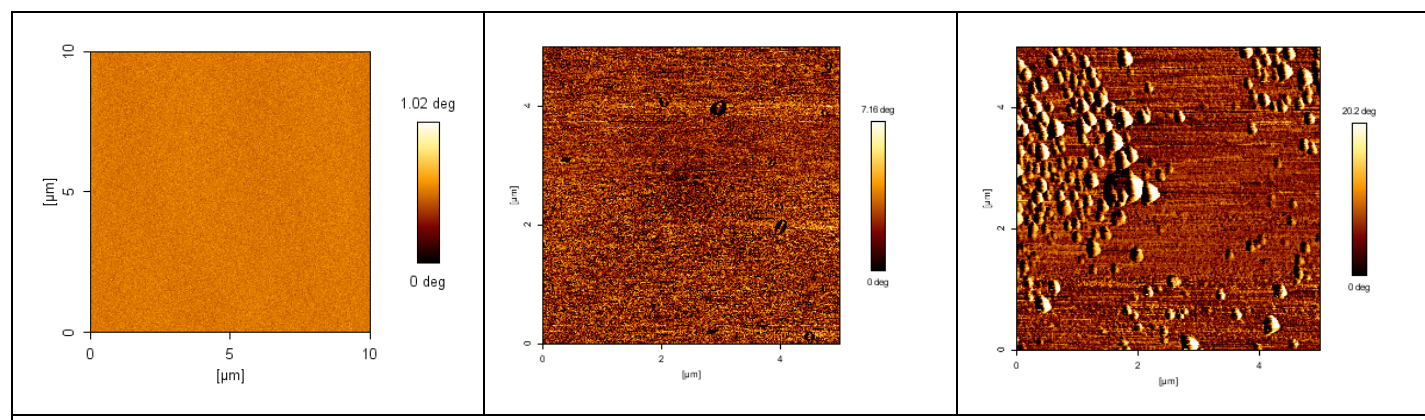

Figure 15. AFM scans summary. A summary of this part of our work, showing side by side AFM scans (phase) of flat surfaces just after the initial air plasma stage (left column), after 5 minutes (middle column) and after 30 minutes (right column) of monomer deposition at 50W. Scan width are $10 \mathrm{um}$ for the air plasma stage sample (left column) and 5um for the other two samples.

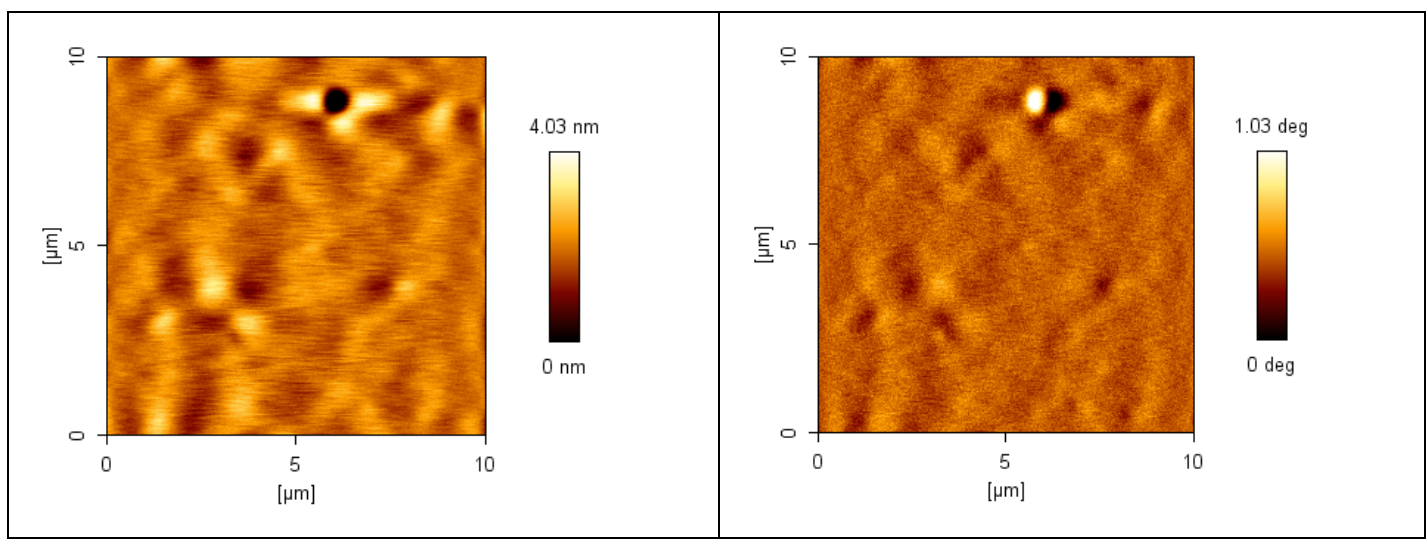




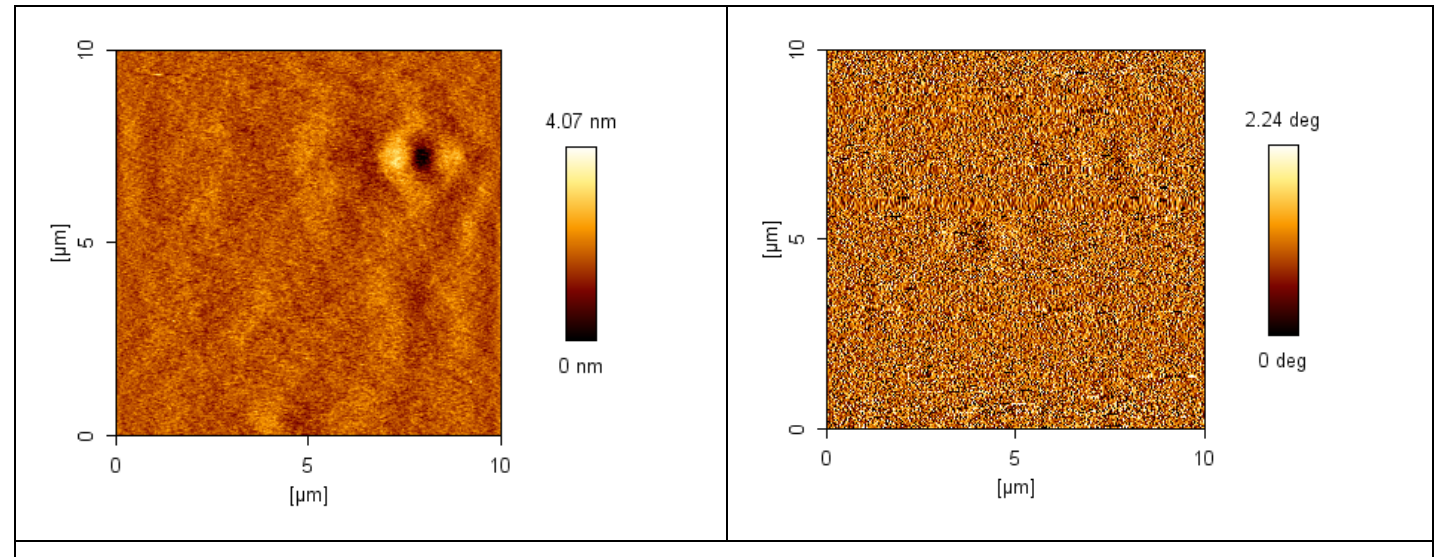

Figure 16. AFM scans plasma 100W, 30 minutes. AFM scans (height on first column and phase on second column) of spin coated PEA ( $4 \%$ in toluene) on glass coverslips. Control surface (spin coated PEA) on first row; surface coated with plasma polymerised PEA at $100 \mathrm{~W}$ for 30 minutes on second row.

\subsubsection{Flaky softer areas on polymerised material}

After deposition in our custom-made reactor, all surfaces with plasma polymerised coatings showed similar features on AFM scans: a homogeneously flat surface covered with polymerised material and randomly distributed flakes or specs of slightly softer polymerised material, more apparent at larger area scans. These flakes are usually a few nanometres high over the surrounding surface and up to a few hundred nanometres wide, although more usually just a few tens of nanometres wide. Experiments (Figure 17) were performed after thorough deep cleaning of all the reactor inner walls, to avoid ablation residues being deposited on the sample surfaces. These experiments confirmed that the deposited materials include a heterogeneous distribution and amount of these softer flakes that varies in different areas of the same samples and in different deposition conditions.

Experiments with a commercial plasma reactor (Figure 18) produced similar surface but with even more variation on the flakes distribution and size with the deposition conditions. 


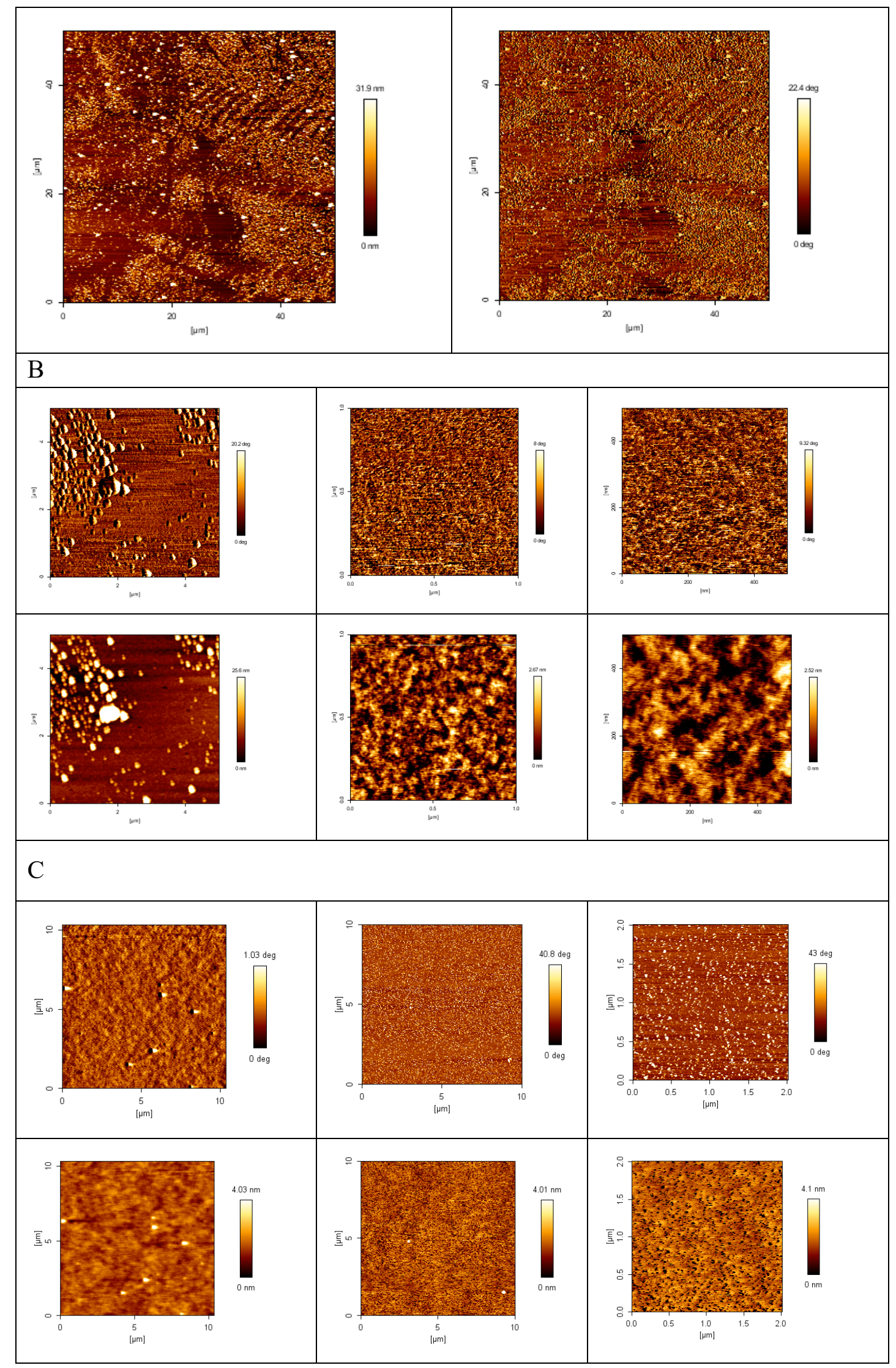


Figure 17. AFM scans plasma 50W, 30 minutes. Height and phase scans of glass coverslip surfaces coated with plasma polymerised PEA at $50 \mathrm{~W}$ for 30 minutes (A and $\mathrm{B}$ ) and at $100 \mathrm{~W}$ for 30 minutes $(\mathrm{C})$.

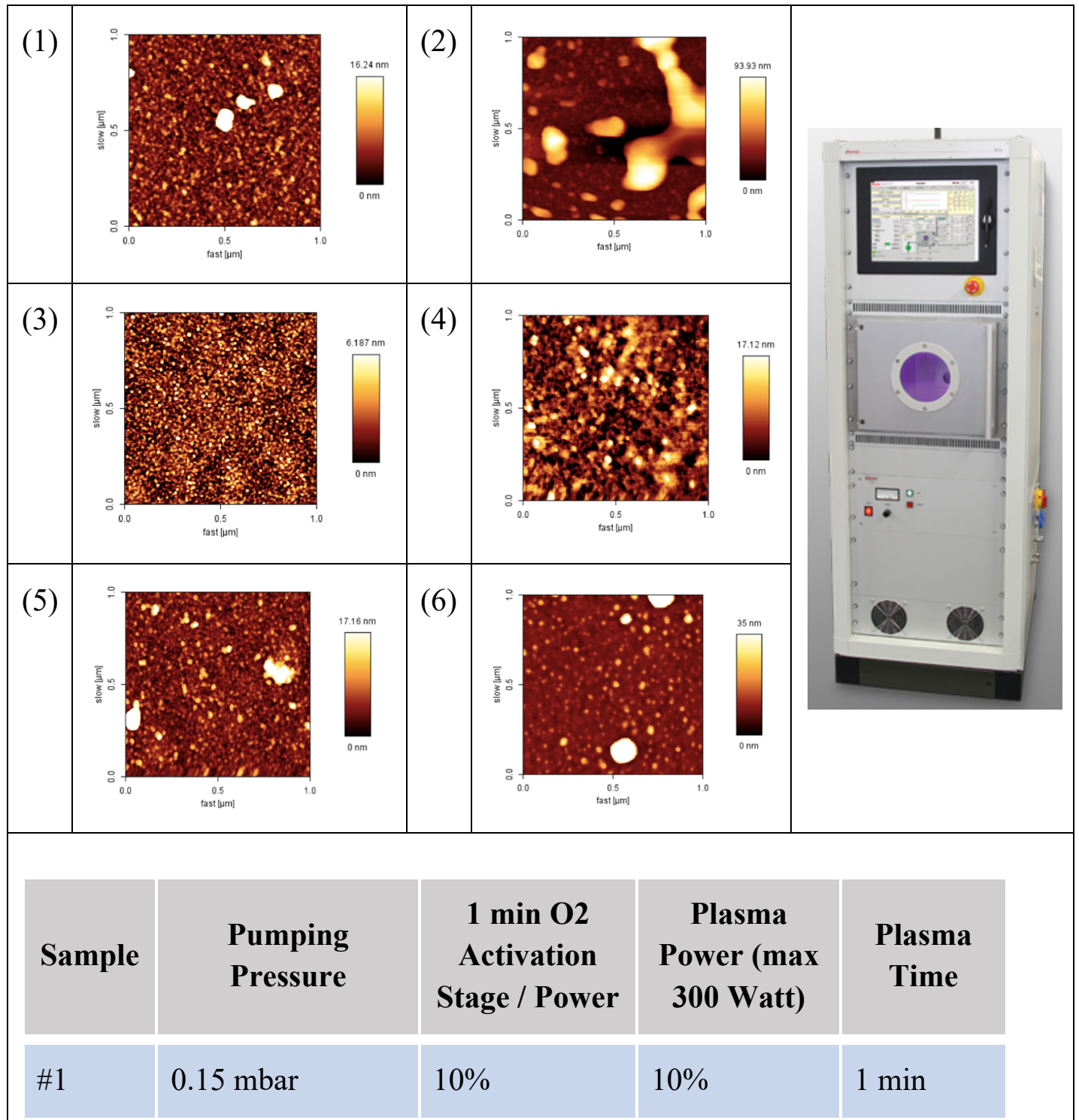




\begin{tabular}{|l|l|l|l|l|}
\hline$\# 2$ & 0.15 mbar & $20 \%$ & $20 \%$ & $1 \mathrm{~min}$ \\
\hline$\# 3$ & $0.15 \mathrm{mbar}$ & $20 \%$ & $20 \%$ & $5 \mathrm{~min}$ \\
\hline$\# 4$ & $0.15 \mathrm{mbar}$ & - & $10 \%$ & $5 \mathrm{~min}$ \\
\hline$\# 5$ & $0.05 \mathrm{mbar}$ & - & $10 \%$ & $5 \mathrm{~min}$ \\
\hline$\# 6$ & $0.05 \mathrm{mbar}$ & - & $5 \%$ & $5 \mathrm{~min}$ \\
\hline
\end{tabular}

Figure 18. Flaky surfaces after plasma deposition. AFM height scans of glass coverslip surfaces coated with plasma polymerised EA in a Diener Electronics Tetra series reactor (right column), with experiment parameters: generator frequencies at 13.56 MHz, monomer flow of $100 \mu \mathrm{l} / \mathrm{min}$, and the settings detailed in the table for each sample, numbered from 1 to 6 .

\subsubsection{Conservation of functional roughness on substrates}

Many biomedical applications, implants and other orthopaedic products in particular, rely on creating and maintaining an interface between the implant and surrounding tissue. This tissue integration can be influenced and enhanced by a number of factors and surface characteristics of the implant materials. Surface roughness and topography (patterned microstructures and nanostructures) have been studied and developed with this goal. This would be the case for instance of nanostructured surfaces used to manipulate stem cell differentiation, designed with pillars around $2 \mu \mathrm{m}$ high and 200 $\mathrm{nm}$ in diameter [17] [47] or functional roughness used on titanium implant surfaces to enhance on bone integration [48].

We prepared different surfaces with controlled roughness or with micro scale functional features and then coated them in our reactor with plasma polymerised EA. We then used AFM to measure the variation in average roughness and the effect on the functional topographical features, to verify conditions where the coating can be applied while maintaining a functional roughness in the supporting material within performance requirements.

\subsubsection{Maintaining functional roughness at the sub-micron scale}

We prepared spin coated amorphous poly(lactic acid) (scPLLA) surfaces on clean glass coverslips and then modified their crystallinity and surface roughness with a thermal treatment. We prepared samples coated with either amorphous PLLA, crystalline PLLA with small spherulites, or crystalline PLLA with big spherulites, as described in the Materials and Methods chapter. 
Spin coated PLLA is amorphous and very flat. After the thermal treatment PLLA becomes crystalline with spherulites that can be controlled in size and height of their ridges. With two different thermal treatments we prepared samples with big spherulites and samples with small spherulites. Both have similar features, crystal edges or ridges that rise a few tens of nanometers, up to around 60 nanometers for the small spherulites and up to around 35 nanometers for the big spherulites (Figure 19).

AFM scans and roughness measurements (Figure 19 to 21) show that our highest energy plasma polymerised EA coating applied, at $100 \mathrm{~W}$ for 30 minutes which has an average thickness of around $300 \mathrm{~nm}$, covers the big spherulite features almost completely. However, the rougher small spherulite surface still maintains a significant fraction of its roughness. This preliminary study demonstrates that our plasma polymerised EA coatings can be applied confidently without altering significantly the substrate surface roughness when features have a main vertical dimension of around one hundred nanometers or more. This opens future developments to fine-tune these coatings on functional surfaces with designed roughness or topographical features.

Further control of substrate roughness, with smaller features preserved, could be achieved using shorter and lower power plasma depositions, as the full set of experiments reported here have been performed with our highest deposition energy. We have shown that much thinner coatings can be produced with lower energies.

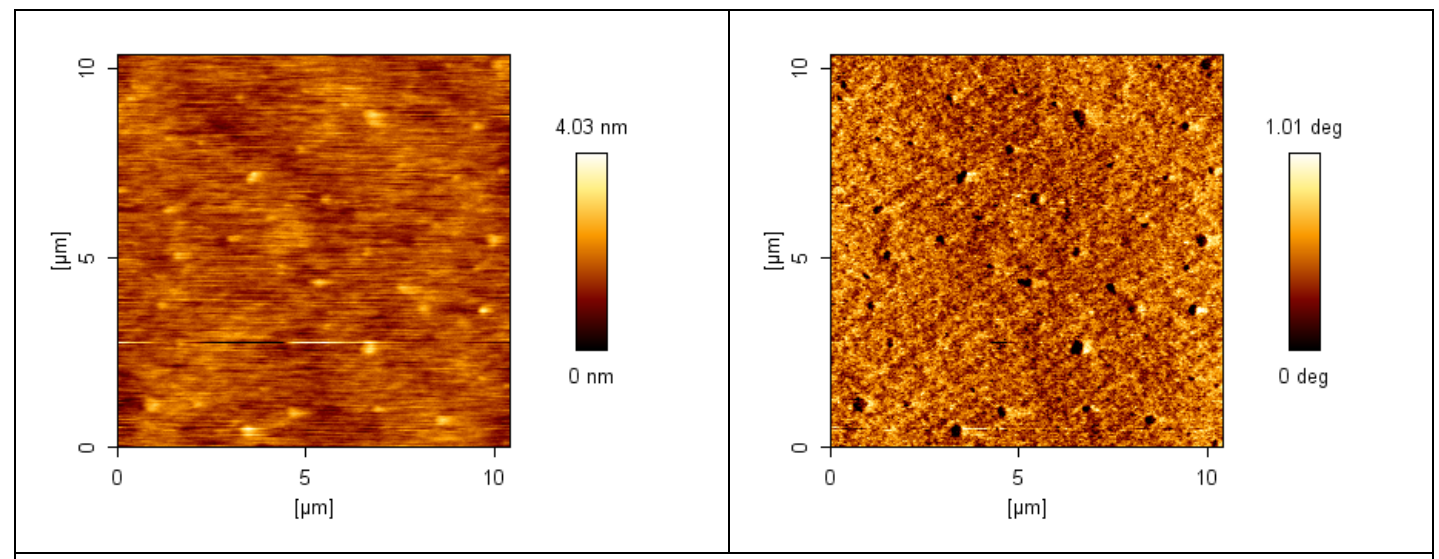

A) Spin coated PLLA on glass coverslips. Amorphous PLLA.

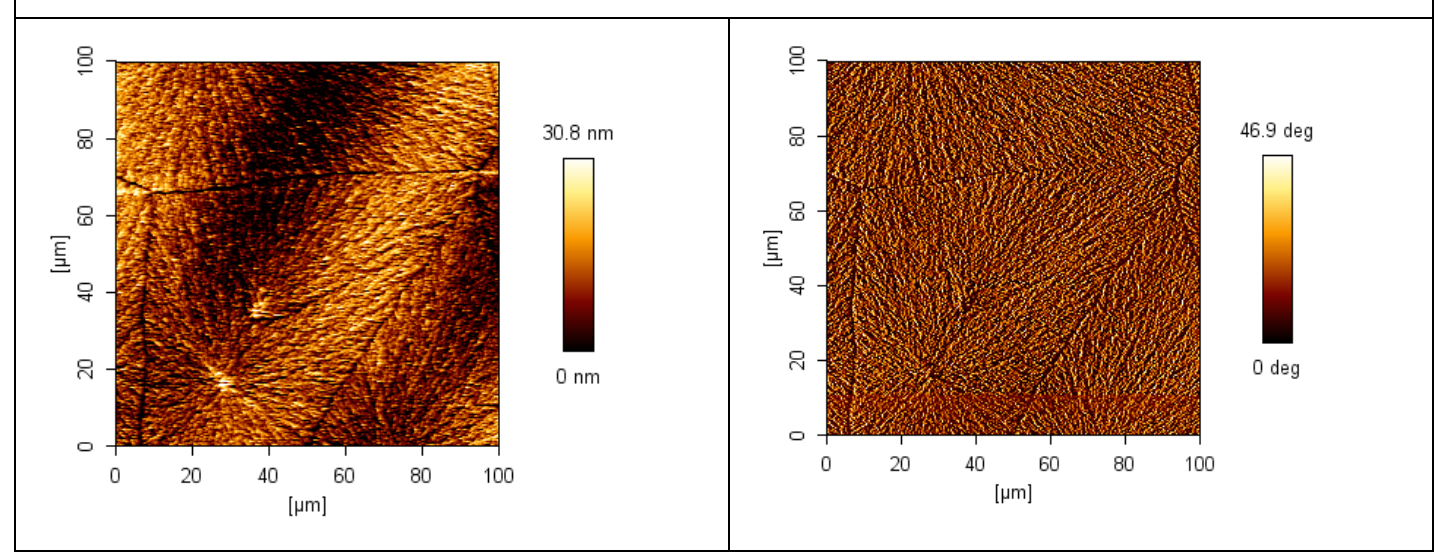




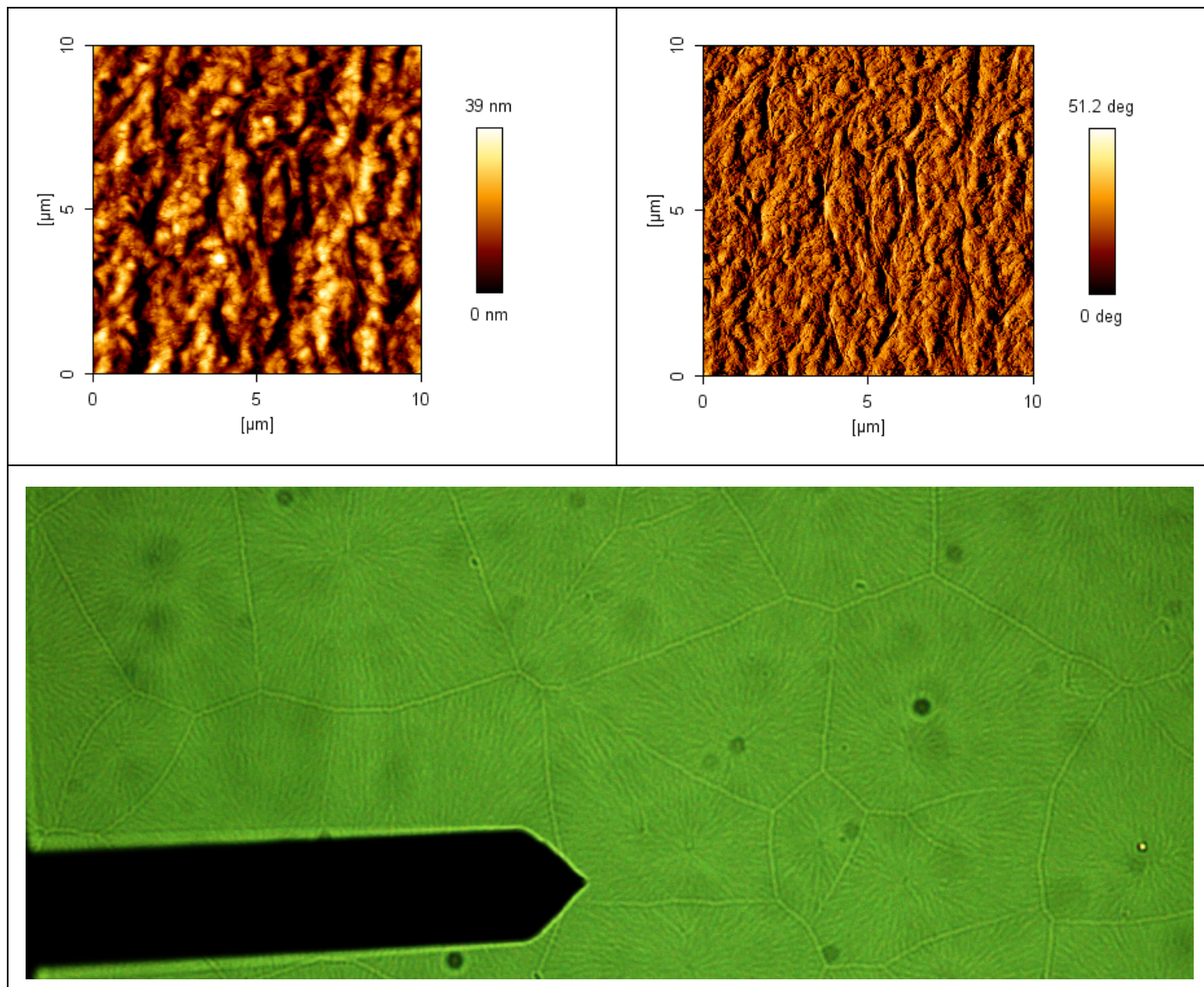

B) Spin coated PLLA on glass coverslips. Thermal treatment - big spherulites.

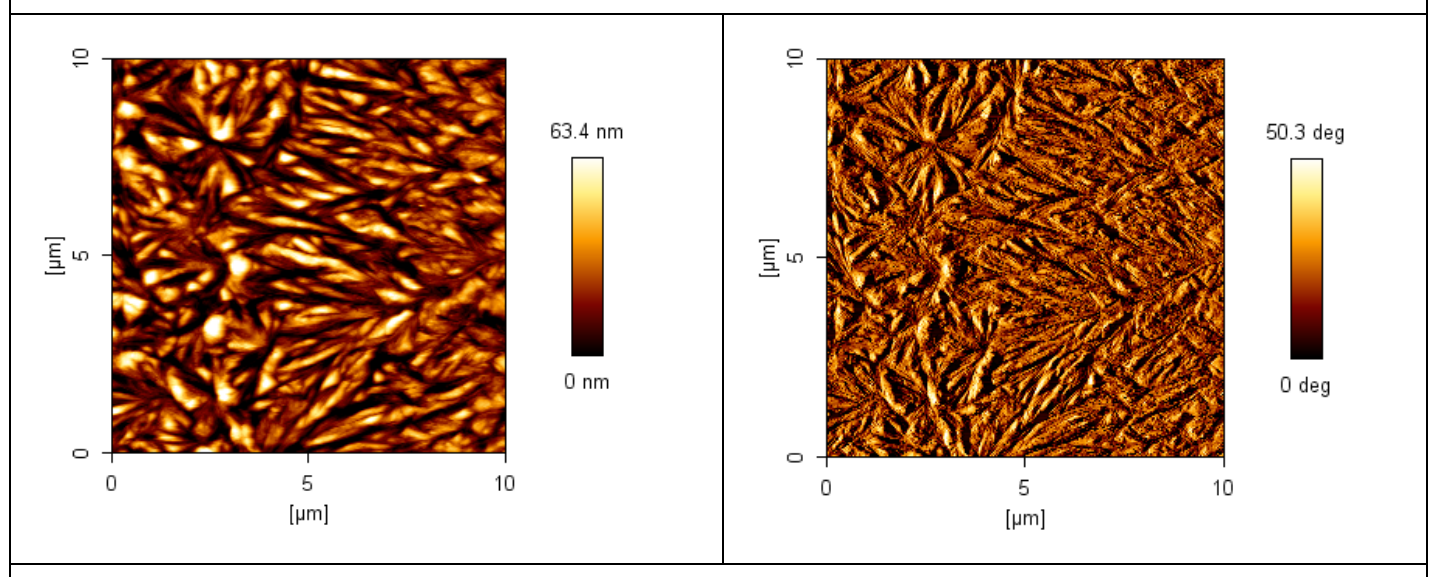

C) Spin coated PLLA on glass coverslips. Thermal treatment - small spherulites 


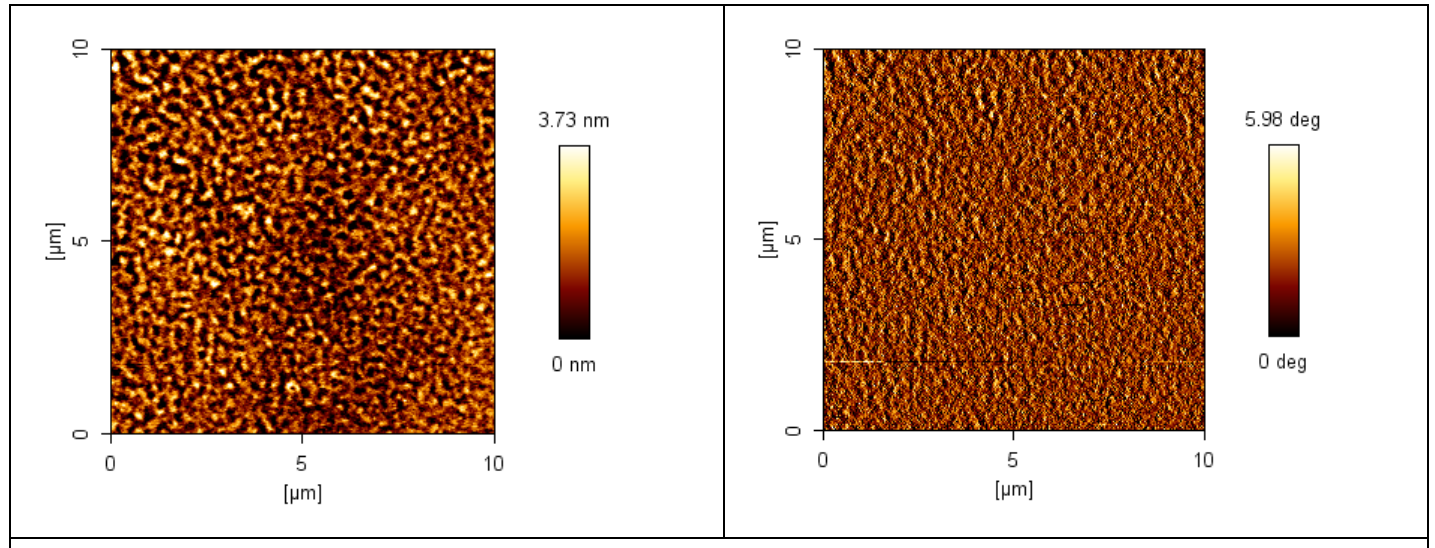

D) Plasma Polymerised EA coating on spin coated PLLA (amorphous).

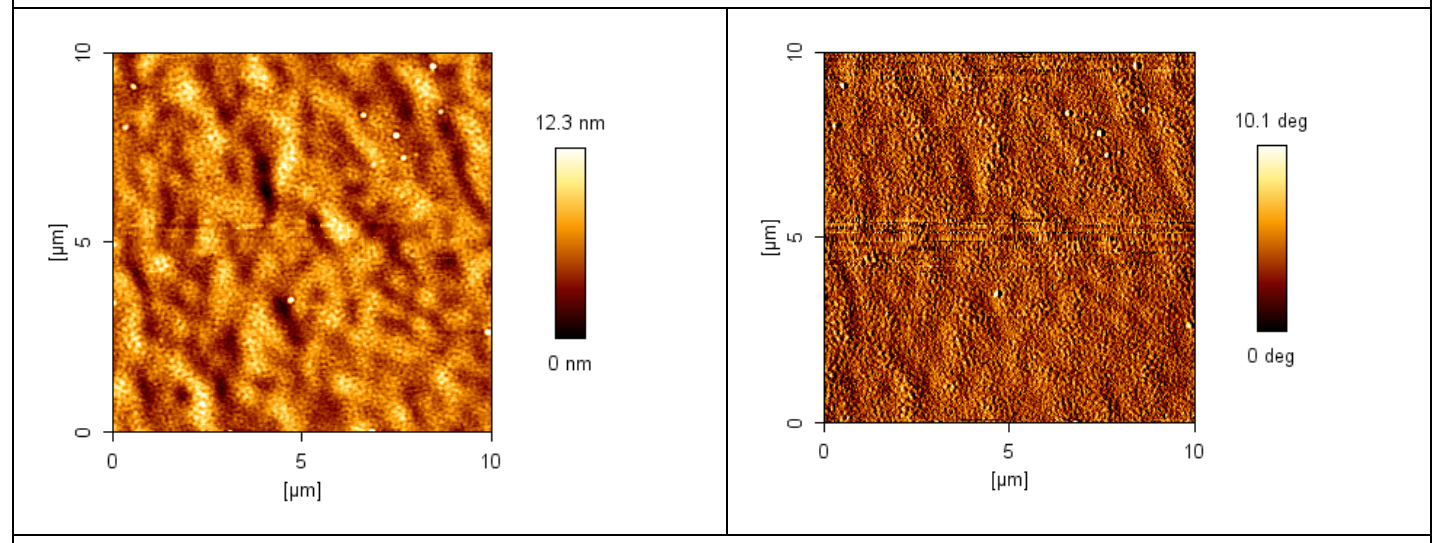

E) Plasma Polymerised EA coating on spin coated PLLA (big spherulites)
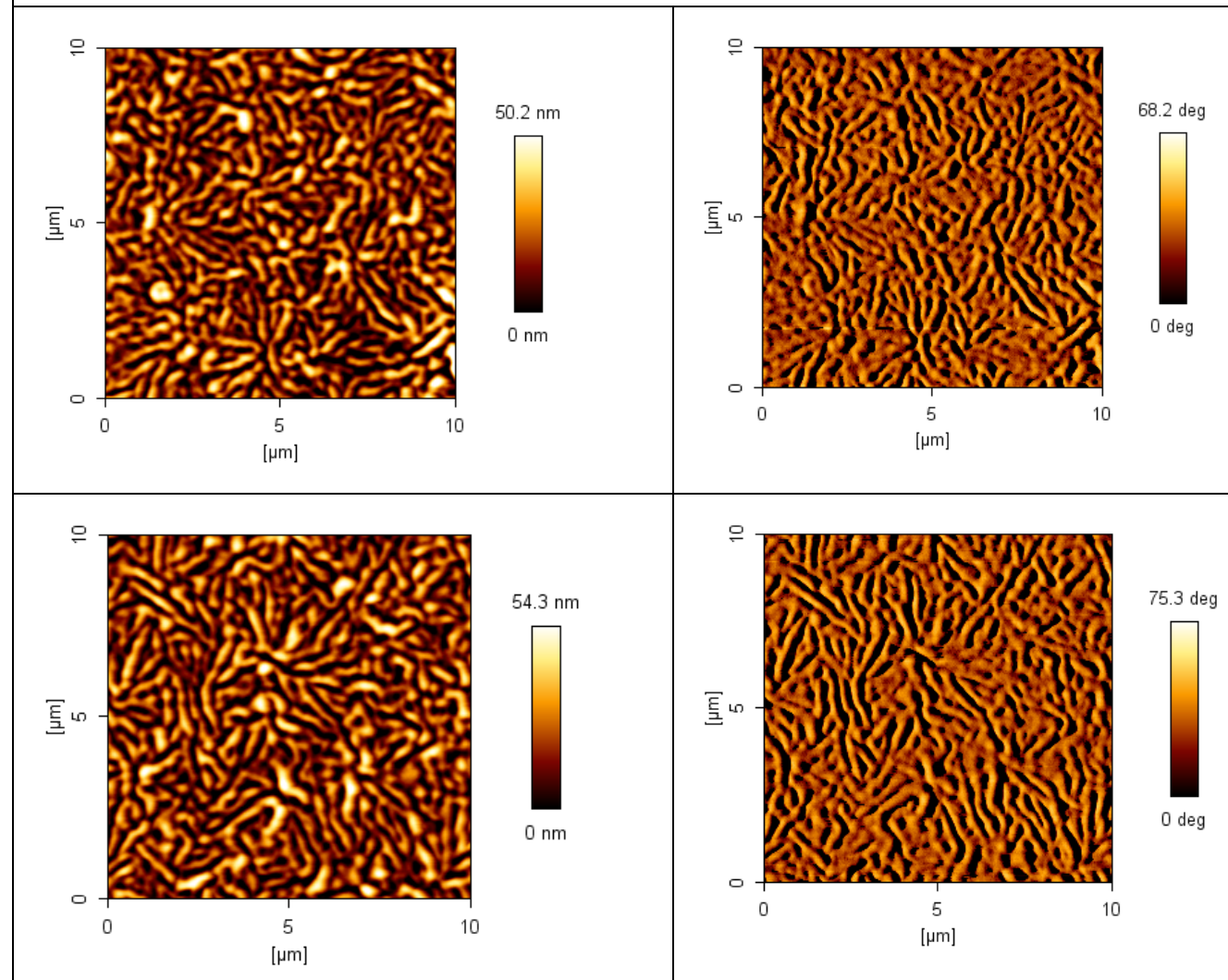
F) Plasma Polymerised EA coating on spin coated PLLA (small spherulites). Two samples are shown.

Figure 19. AFM scans of spin coated PLLA surfaces with controlled roughness. All scans with height on left column, phase on right column) A), B) and C) are controls, without plasma polymerized coating, for amorphous PLLA, crystalline PLLA with big spherulites, and crystalline PLLA with small spherulites respectively. D), E) and F) are coated samples at $100 \mathrm{~W}$ and for 30 minutes of duration of the plasma polymerization stage, for amorphous PLLA, crystalline PLLA with big spherulites, and crystalline PLLA with small spherulites respectively.

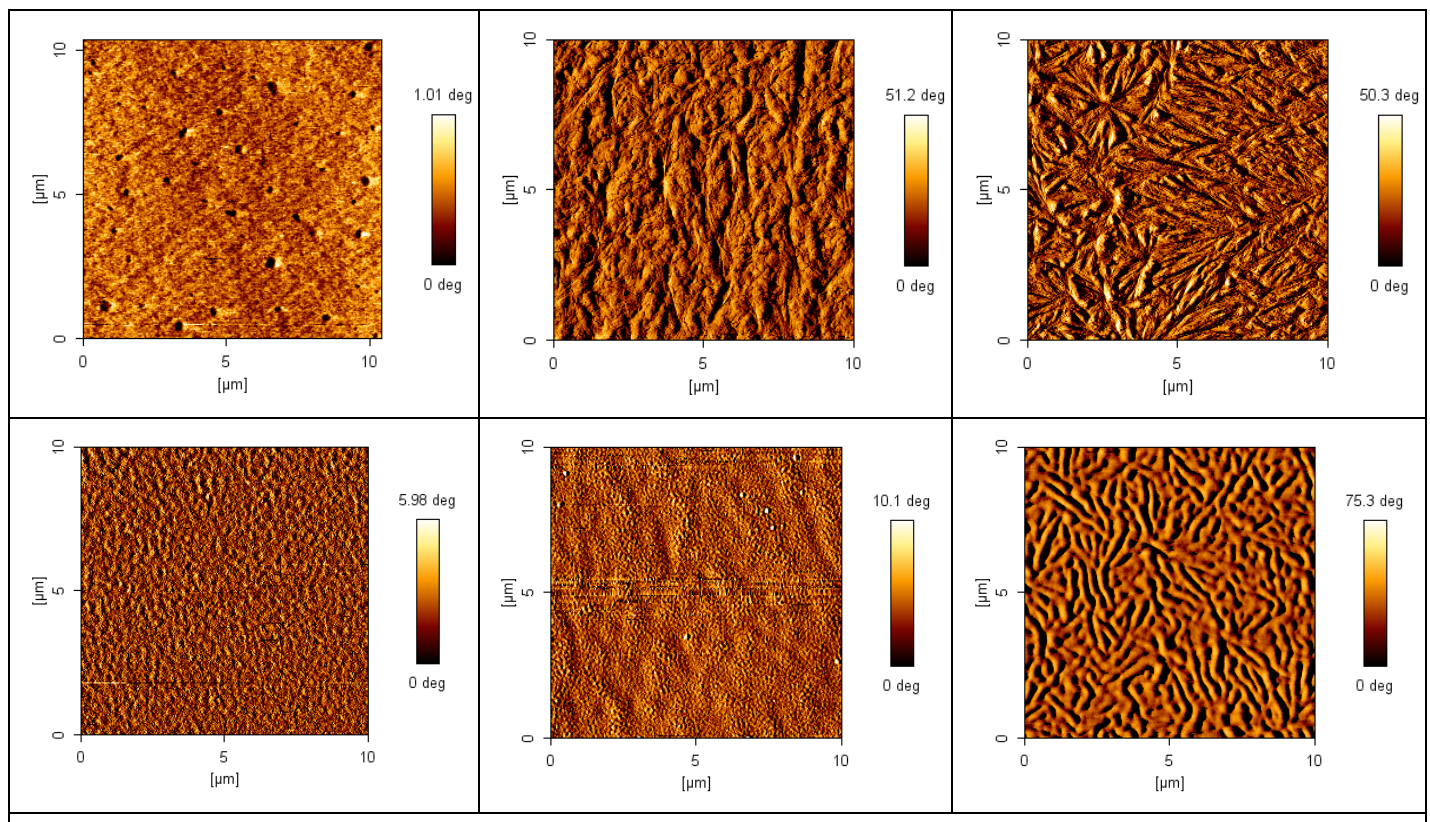

Figure 20. AFM phase scans of spin coated PLLA surfaces with controlled roughness. First row are controls, without plasma polymerized coating, second row are coated samples at $100 \mathrm{~W}$ and for 30 minutes of duration of the plasma polymerization stage. First column is amorphous PLLA, second column crystalline PLLA with big spherulites, and third column is crystalline PLLA with small spherulites. 


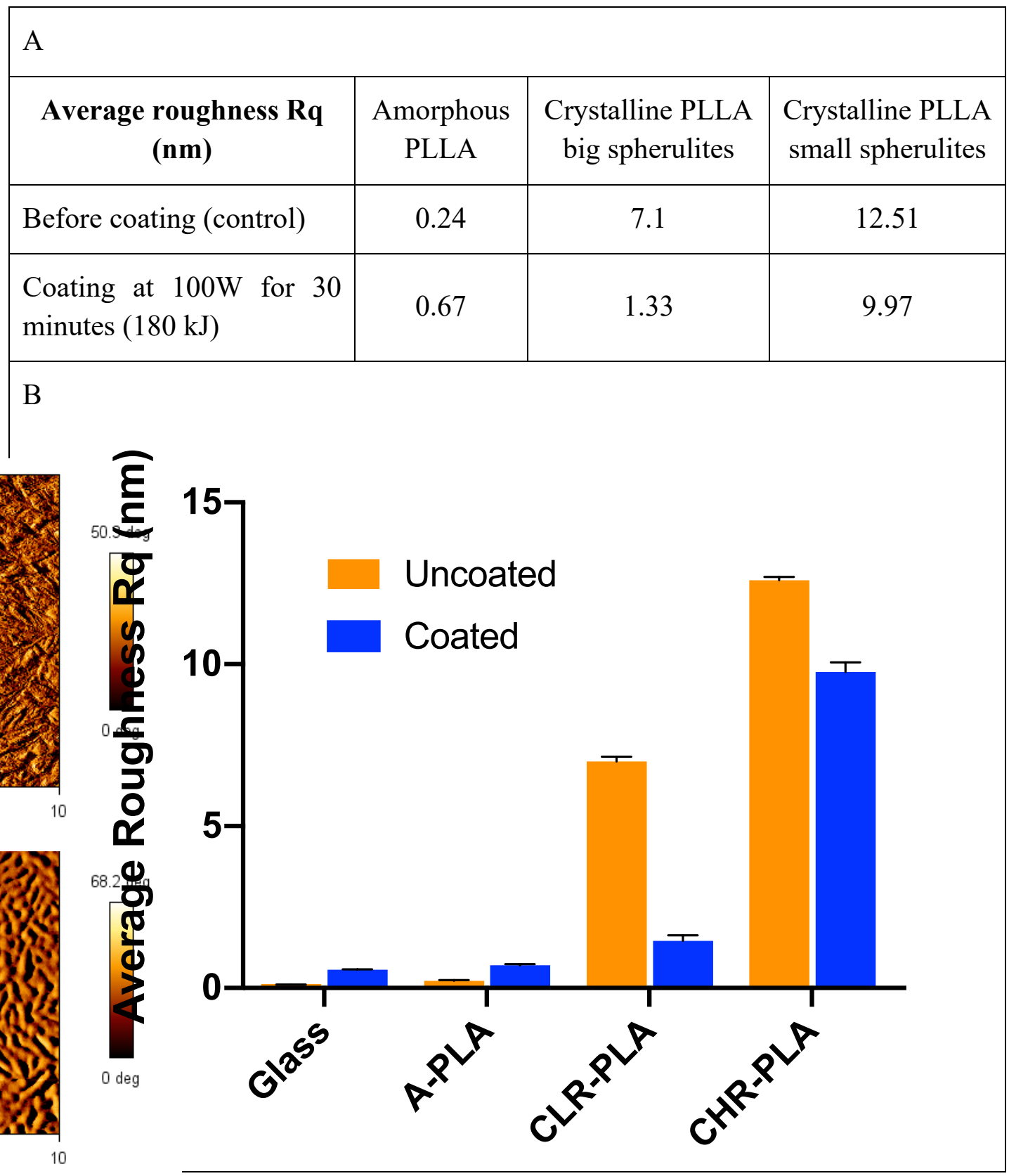


Figure 21. Roughness on coated rough samples. Measured average roughness $\mathrm{Rq}$ $(\mathrm{nm})$ on $10 \times 10 \mu \mathrm{m}$ AFM scans before and after coating with plasma polymerised EA $(180 \mathrm{~kJ})$. Surfaces are glass, amorphous PLA (A-PLA), crystalline low roughness PLA (CLR-PLA), and crystalline high roughness PLA (CHR-PLA). CHR-PLA surfaces with higher roughness showed a decrease in roughness after coating but much smaller than on CLR-PLA, compared to substrate uncoated surfaces, demonstrating that a functional roughness can be maintained for CHRPLA samples at these coating conditions. (A) Table with measured values, and (B) graph visually comparing results.

\subsubsection{Control of coating deposition over hyper-rough surfaces}

We tested the effect on roughness by the plasma polymerised coatings on surfaces with features with functional dimensions well under one hundred nanometres, such as nanopits, spikes, or nanowires. We looked to identify coating conditions that can be used to successfully coat these surfaces while controlling the function of their underlaying features. High aspect ratio surfaces like these are investigated in the literature for their effect on cell adhesion mechanisms and ultimately cell behaviour, for instance to influence stem cell fate [17] or to prevent bacterial biofilm formation but allow human cells growth [49] [50].

In a first test, we used a polycarbonate surface with a nanopattern of etched pits kindly provided by Dr Paul Reynolds and Prof Nikolaj Gadegaard at the University of Glasgow. These nanopatterned surfaces have been used in a separate wider study to show that tuned disorder in nanotopography stimulates osteogenesis [51]. We applied a plasma polymerised EA very thin coating, at low power $(30 \mathrm{~W})$ and short duration of treatment $(60 \mathrm{~s})$ using a commercial Diener plasma reactor and confirmed with AFM scans that the coating doesn't plug or covers over the nanopits at all (Figure 19).

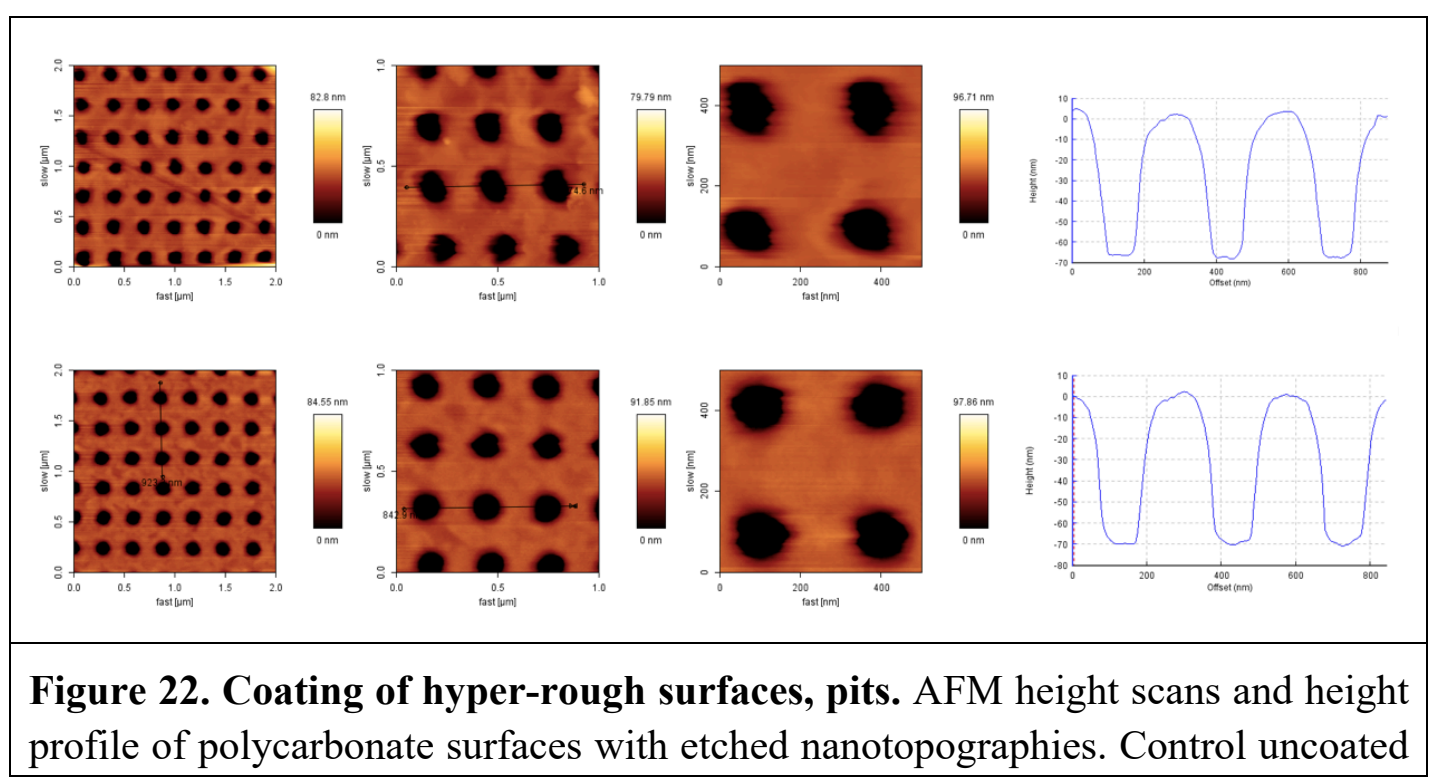


surfaces (first row) and surfaces coated with plasma polymerised EA using a Diener Electronics Tetra series plasma reactor with experiment parameters as in sample \#1 of Figure 14 (30W, 60s plasma stage)

In a second test, Titanium substrates with $\mathrm{TiO} 2$ nanowires grown on the surface were coated with plasma polymerised EA. These ultra-rough surfaces were prepared by colleagues at the University of Glasgow as part of a different study to identify optimal nanopatterns for both bone remodelling and for reducing risk of bacterial infection. We coated the metallic samples with thin plasma polymerised EA coatings, at low power and short duration of treatments. In this test the goal was to test if our coating system could be controlled to provide these surfaces with the additional functionality of our coating while maintaining the functional sharpness of the nanofeatures. Scanning electronic microscopy (SEM) images showed no perceptible effect on the nanowire coated surfaces (Figure 23). A more extensive characterization of the physical characteristics and biological performance of these spiky coated surfaces is being done by colleagues at the University of Glasgow.
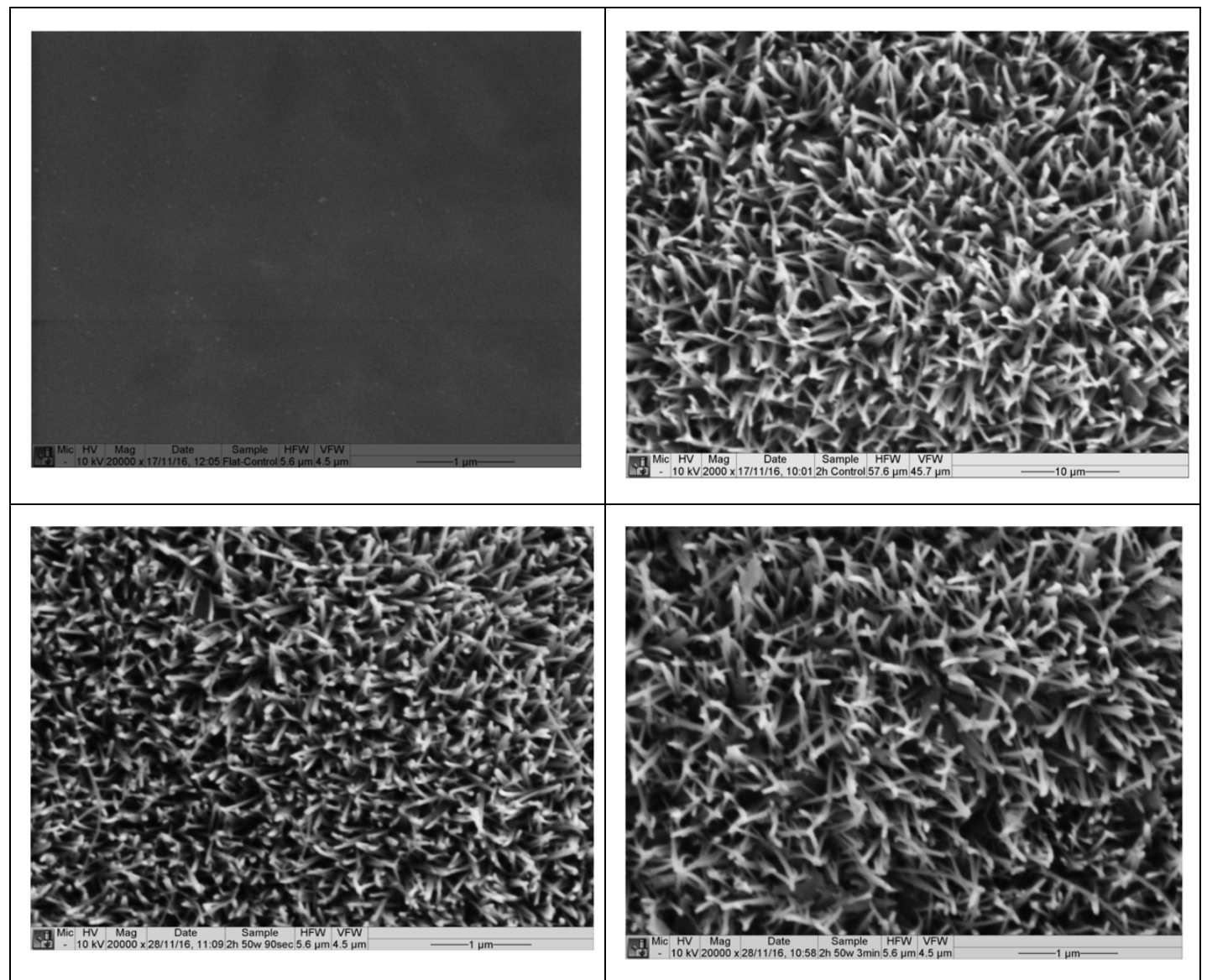

Figure 23. Coating of hyper-rough surfaces, nanowires. SEM images of titanium surfaces with sharp $\mathrm{TiO} 2$ nanowires grown on the surface. On top row, flat $\mathrm{Ti}$ surface (left) and Ti surface with $\mathrm{TiO} 2$ nanowires grown for 2 hours (right). On bottom row, same surface ( $\mathrm{TiO} 2$ nanowires grown for $2 \mathrm{~h}$ ) coated with plasma polymerised EA at $50 \mathrm{~W}$ for $90 \mathrm{~s}$ (left) and for 180s (right) 


\subsubsection{Water contact angle of plasma coated surfaces}

Measurement of the water contact angle (WCA) of surfaces coated with plasma polymerised ethyl acrylate, on control glass or polymer surfaces, allowed for a further understanding of the characteristics of the plasma coatings, i.e. surface energy, hydrophilicity and free radicals, with varying plasma polymerisation conditions.

We produced several substrate surfaces to coat with plasma polymerised ethyl acrylate, including glass, and spin coated poly L-lactic acid (PLLA) prepared with two degrees of crystallinity (small spherulites and big spherulites). Control surfaces also tested included spin coated PEA on glass and spray coated PEA on spin coated PLLA.

We measured the static contact angle (SCA) at different time points after plasma coating (Figure 24), from immediately after plasma coating up to 14 days of storage under dry cupboard conditions. We verified that wettability of the surfaces showed by SCA remains fairly unchanged during this time frame. This means that surfaces coated with our system retain functionality and future applications of this technology can use materials that have been stored, before in vivo or in vitro use, without changing functionality. We additionally checked the effect of water immersion on the SCA of the plasma polymerised EA coated surfaces (Figure 25), showing an increase in hydrophobicity of the tested samples.

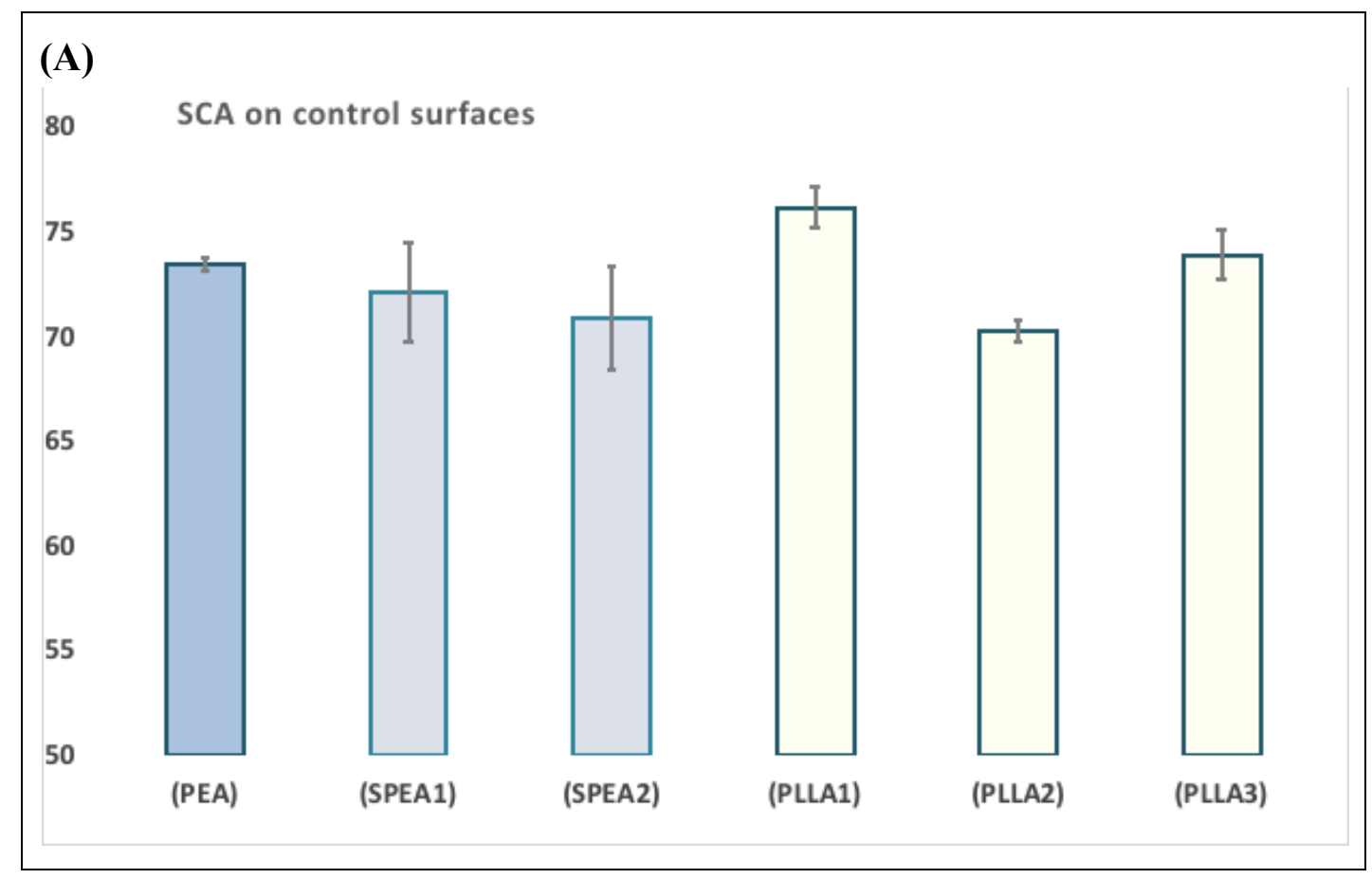




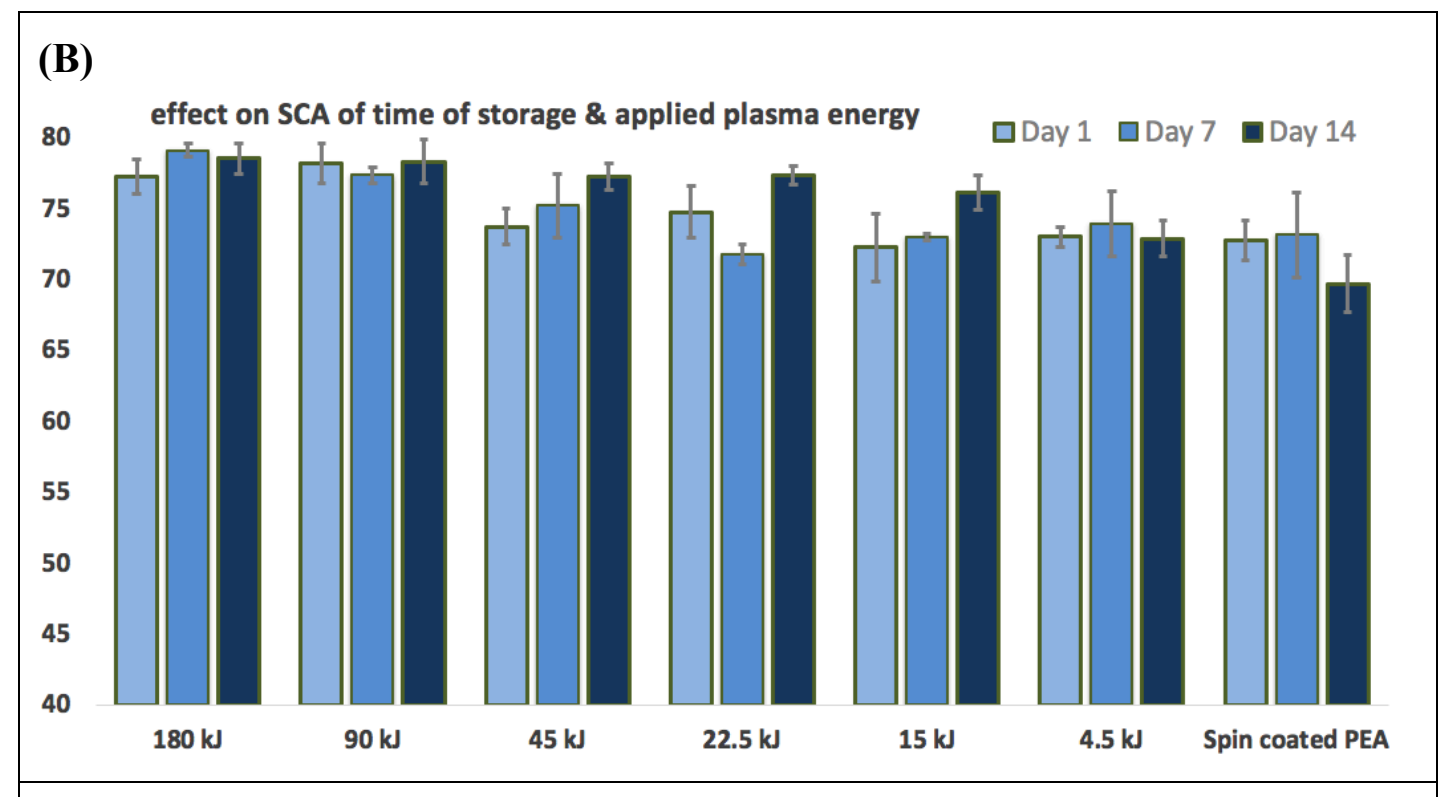

Figure 24. Water contact angle measurements. Compared to controls and by energy applied. (A) Static contact angle measured on control surfaces. All surfaces dried under vacuum and measurements performed within $24 \mathrm{~h}$ after surface preparation. (PEA) is spin coated PEA on glass. (SPEA1) and (SPEA2) are spray PEA on crystalline PLLA, with big spherulites and with small spherulites respectively. (PLLA1), (PLLA2) and (PLLA3) are amorphous PLLA, crystalline with small spherulites PLLA, and crystalline with big spherulites PLLA, respectively, prepared by spin coating and heat for the crystalline samples.

(B) SCA at three time points after coating glass with plasma polymerised EA. Plasma deposition conditions by energy applied, from $180 \mathrm{~kJ}(100 \mathrm{~W}$ and $30 \mathrm{~min}$ of plasma) to $4.5 \mathrm{~kJ}$ ( $15 \mathrm{~W}$ and $5 \mathrm{~min})$.

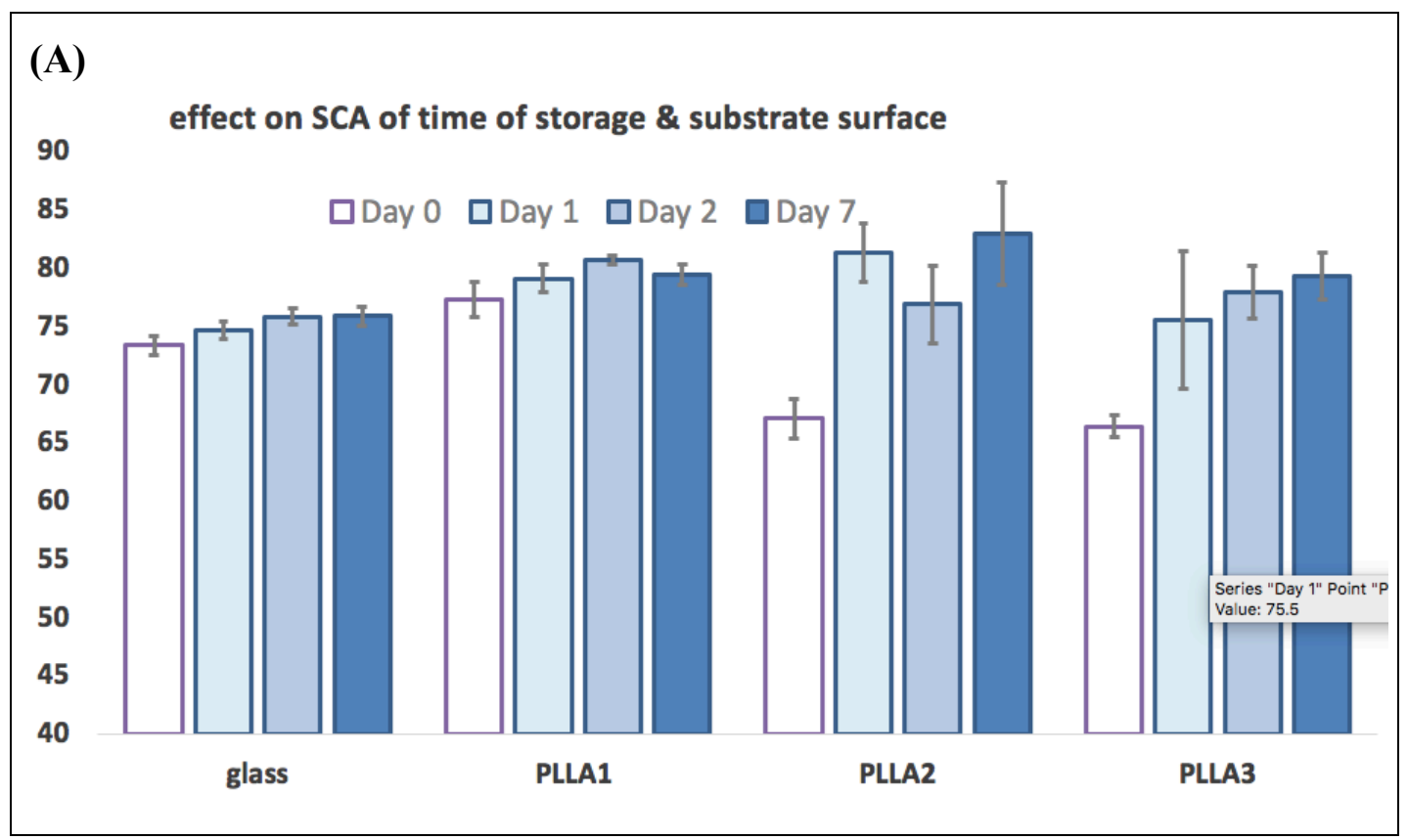




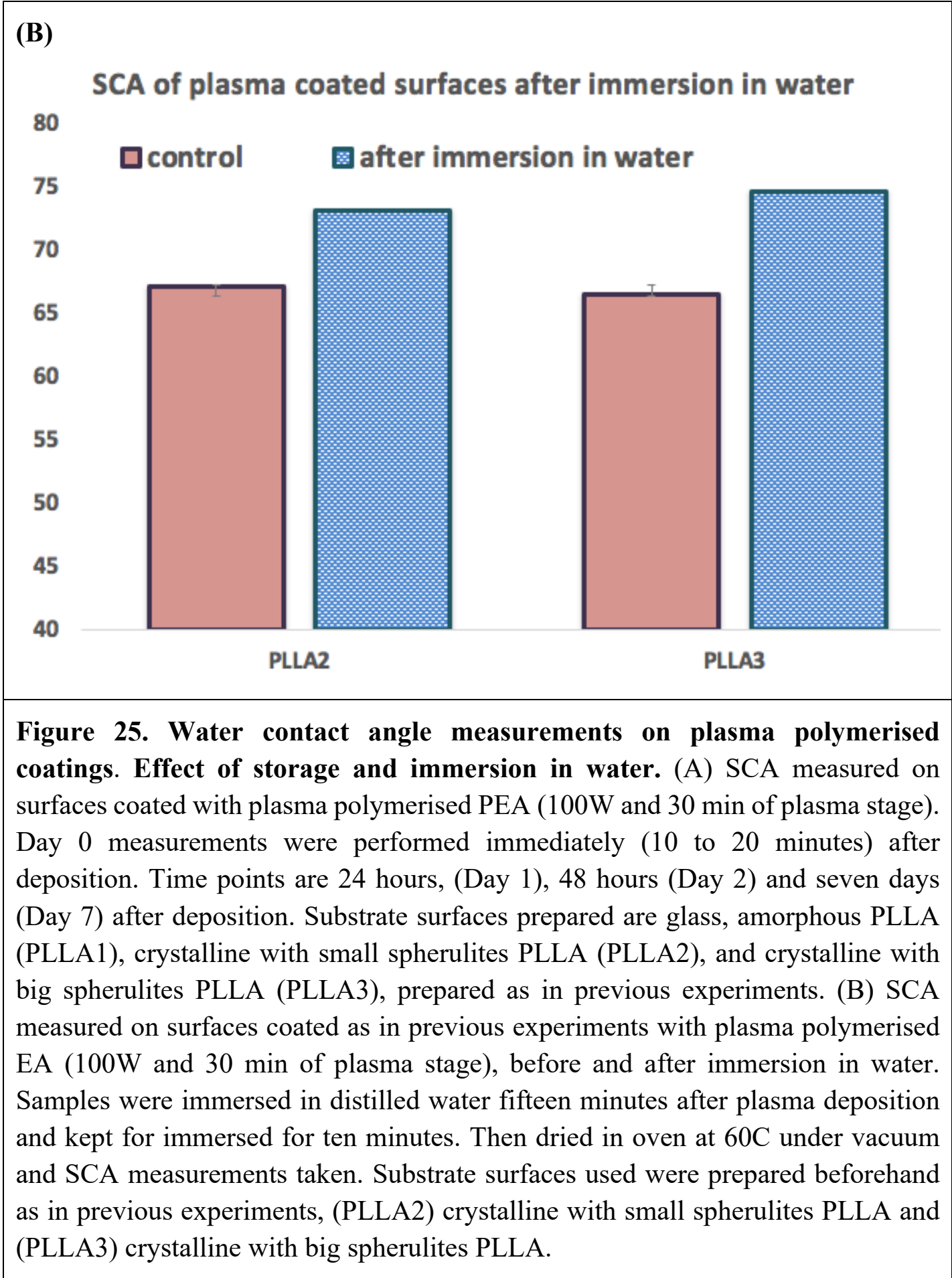

Overall, we have seen that plasma polymerized PEA surfaces are quite hydrophobic, with WCAs around $70^{\circ}$ to $80^{\circ}$ which are comparable to even if slightly higher than SC-PEA coatings. Literature shows that cells effectively adhere onto surfaces presenting moderate wettability, with water contact angles in the range of $40^{\circ}$ to $70^{\circ}$ [52][53]. The plasma polymerized coatings however, as many other synthetic surfaces, present WCA above this optimal range. Adsorption of a protein coating on the plasma polymerized surface can improve wettability for the intended biomedical applications. 


\subsubsection{Chemical analysis of plasma polymerised materials - XPS}

X- ray photoelectron spectroscopy (XPS) measurements were performed to study the chemical composition on the PEA deposited surfaces. SC-PEA was analysed via peak fitting of the carbon (C1s) and oxygen (O1s) spectra (Figure 26), showing peak positioning and binding ratios similar to those found in literature.

$\mathrm{C} 1 \mathrm{~s}$ spectra were peak fitted with respect to the primary hydrocarbon backbone signal at $285 \mathrm{eV}$ the other three carbon moieties respectively: C-COOR $(+0.4 \mathrm{eV})$; $\mathrm{C}-\mathrm{O}(+1.6$ $\mathrm{eV})$; $\mathrm{O}-\mathrm{C}=\mathrm{O}(+3.9 \mathrm{eV})$, corresponding to chemical structure inset in figure $23 \mathrm{~A}$.

In O1s spectra two oxygen moieties, $\mathrm{C}=\mathrm{O}(\sim 532.1 \mathrm{eV})$ and $\mathrm{C}-\mathrm{O}-\mathrm{C}(\sim 533.5 \mathrm{eV})$ were found. The $\mathrm{C} 1 \mathrm{~s}$ and $\mathrm{O} 1 \mathrm{~s}$ core level spectra obtained for plasma PEA coatings varied dramatically from SC-PEA, possessing lower concentrations of ester and carboxyl carbon binding environments, signified by loss of peaks in the spectra and definition of both corresponding oxygen peaks.

From the carbon spectra, it can be noted that while the peak found at $+1.6 \mathrm{eV}$ from the backbone has almost entirely diminished the $+3.9 \mathrm{eV}$ peak is proportionally retained when plasma polymerised at lower energies. This relationship can be further observed in the oxygen spectra, which shows decreasing oxygen peaks correlating to the loss of these chemical sidechains. This translates to increased proportions of primary carbon binding relating to the PEA backbone, further enhancing and defining the $285 \mathrm{eV}$ peak. However, the $\mathrm{C} 1 \mathrm{~s}$ spectra for all pPEA samples showed peaks at 285 $\mathrm{eV}$ (carbon-carbon backbone bond) and also consistently showed retention of small shoulder peaks at $288.9 \mathrm{eV}$ which corresponds to the $\mathrm{C} 1 \mathrm{~s}$ binding energy in carbonyl groups $(\mathrm{C}=\mathrm{O}, \mathrm{C} 1 \mathrm{~s}$ binding energy 288.0$)$ and acid groups $(\mathrm{O}=\mathrm{C}-\mathrm{O}, \mathrm{C} 1 \mathrm{~s}$ binding energy 289.0), suggesting the pPEA coatings maintains some of the moieties characteristic of PEA (SC-PEA).

This retention of functional groups is observed to relate to the polymerisation conditions, suggesting that increased energy directly influences the mechanism by which these peaks are lost, increasing the percentage of carbon backbone and decreasing the functional group composition on the top $10 \mathrm{~nm}$ of sample.

This chemical modification is characteristic of plasma discharge, resulting in monomer fragmentation, chain branching, cross linking and partial loss of functional groups during polymerisation [54] [55]. More specifically, fragmentation, resulting from higher experimental powers producing a higher frequency and energy of electron impacts onto the polymerising EA monomer, would provide an apt explanation for the resulting chemical variations of plasma PEA. Therefore, we are able to conclude that plasma polymerisation produces oxygen deficient PEA coatings, with the level of remaining oxygen based functional groups directly depending on applied energy.

Three samples and three different areas per sample were scanned per deposition condition, with examples in Figure 27. We deposited the plasma polymerized coatings on glass and also on spin coated PLLA, both amorphous and crystalline (rougher) 
PLLA surfaces. With the XPS spectra of these samples (Figures 28 and 29) we confirmed the that the thickness of the deposited coatings $(\approx 300 \mathrm{~nm})$ guarantees that the XPS spectra are due to pPEA and not to the underlying substrate, as the sensitivity of XPS is $\approx 10 \mathrm{~nm}$.

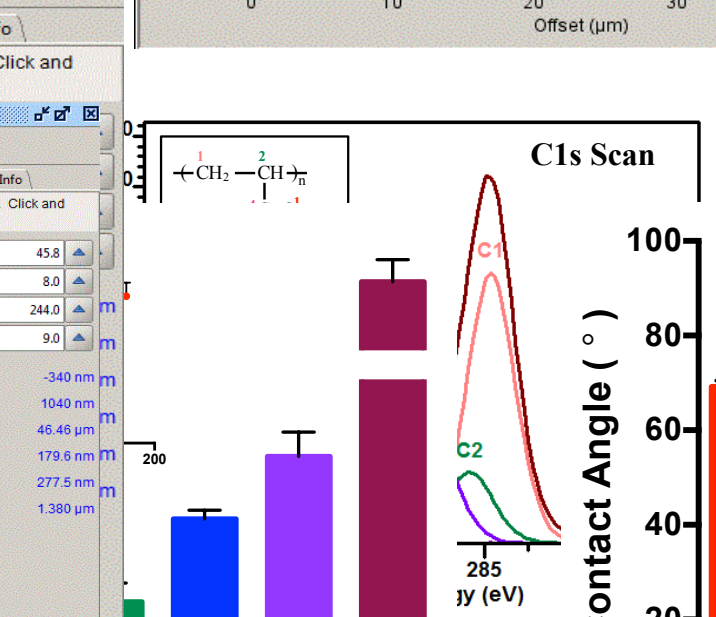

B

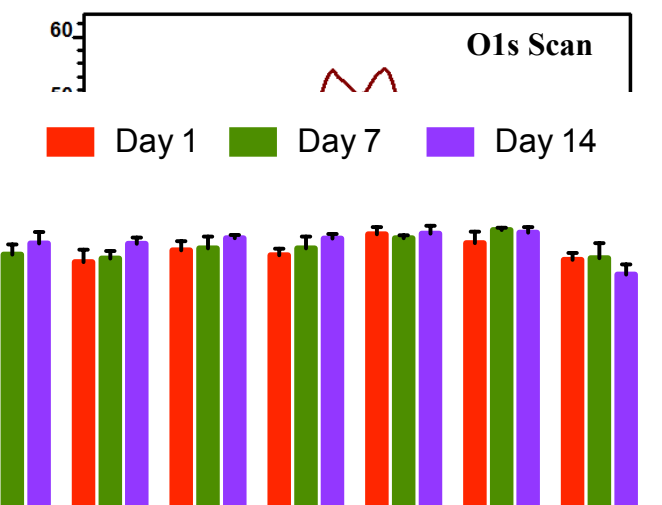

C

$\mathrm{D}$
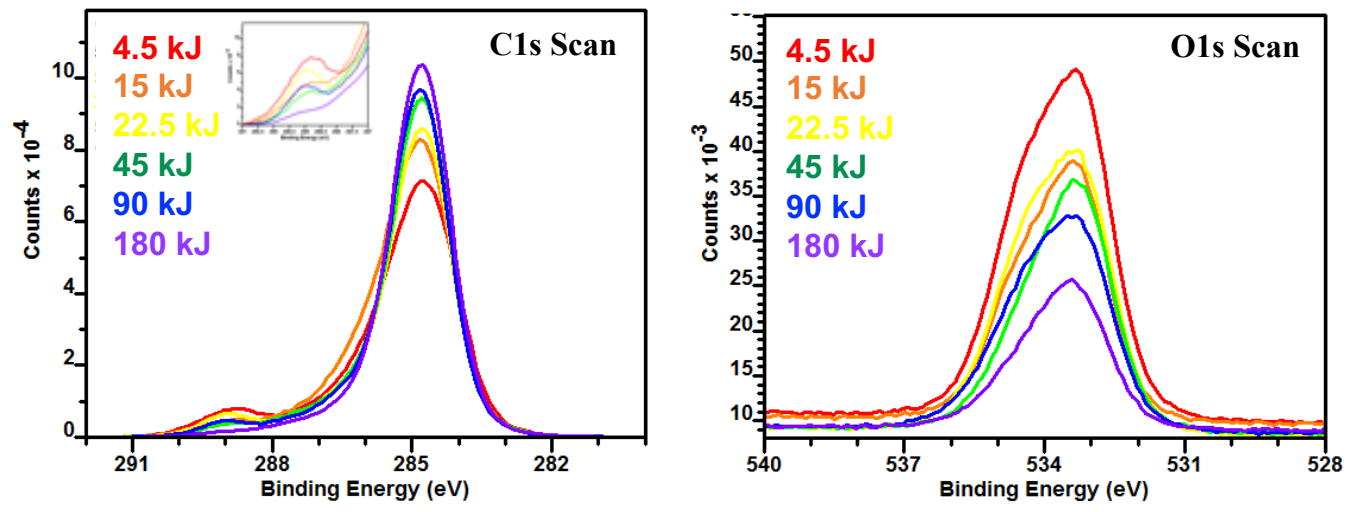

Figure 26. Chemical composition of PEA surfaces taken by XPS analysis, by plasma deposition energy. (A, B) $\mathrm{C} 1 \mathrm{~s}$ and $\mathrm{O} 1 \mathrm{~s}$ core-level spectra of control SCPEA with fitted peaks which represent binding conformations of carbon and oxygen atoms on the top $10 \mathrm{~nm}$ of the sample surfaces. The inset shows chemical structure of PEA, with labelled carbon and oxygen atoms corresponding to components as indicated on $\mathrm{C} 1 \mathrm{~s}$ and $\mathrm{O} 1 \mathrm{~s}$ scans. (C, D) Overlaid $\mathrm{C} 1 \mathrm{~s}$ and $\mathrm{O} 1 \mathrm{~s}$ core-level spectra of pPEA samples produced at varying energies, showing generally that increasing energy (power $\mathrm{x}$ time of deposition) results in decreasing ester and carboxyl side chains on the produced PEA. The inset in C shows the peak at $289 \mathrm{eV}$. 


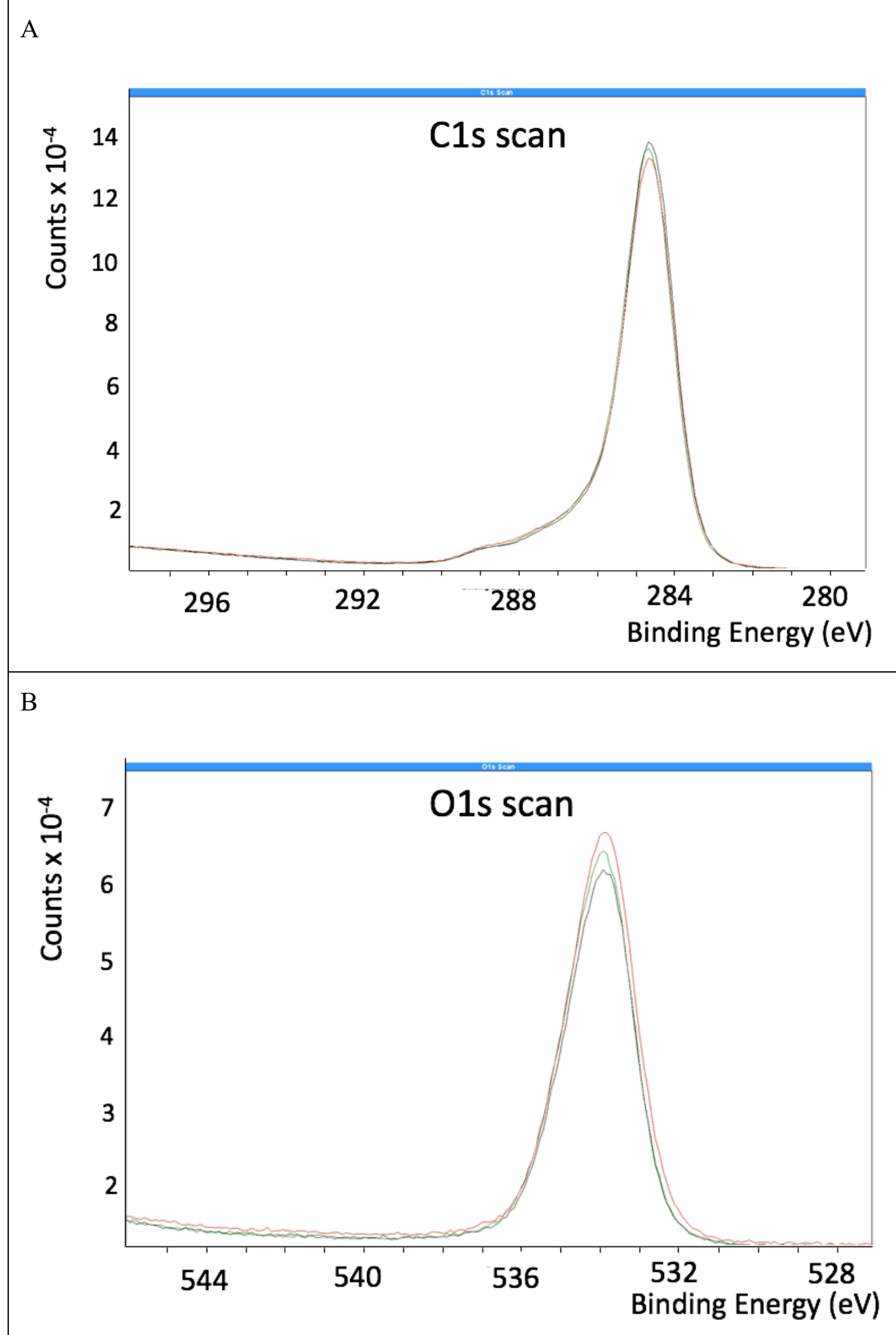

Figure 27. XPS analysis of PEA surfaces, variation between samples. XPS spectra from three samples treated under the same experimental conditions, one selected area for each sample. Samples were prepared coating glass coverslips with plasma polymerised EA, at $100 \mathrm{~W}$ of power and a duration of 30 minutes $(\mathbf{1 8 0} \mathbf{k J})$ 
of the monomer plasma stage. (A) XPS spectra around the binding energy for $\mathrm{C} 1 \mathrm{~s}$, and (B) XPS spectra around the binding energy for O1s.

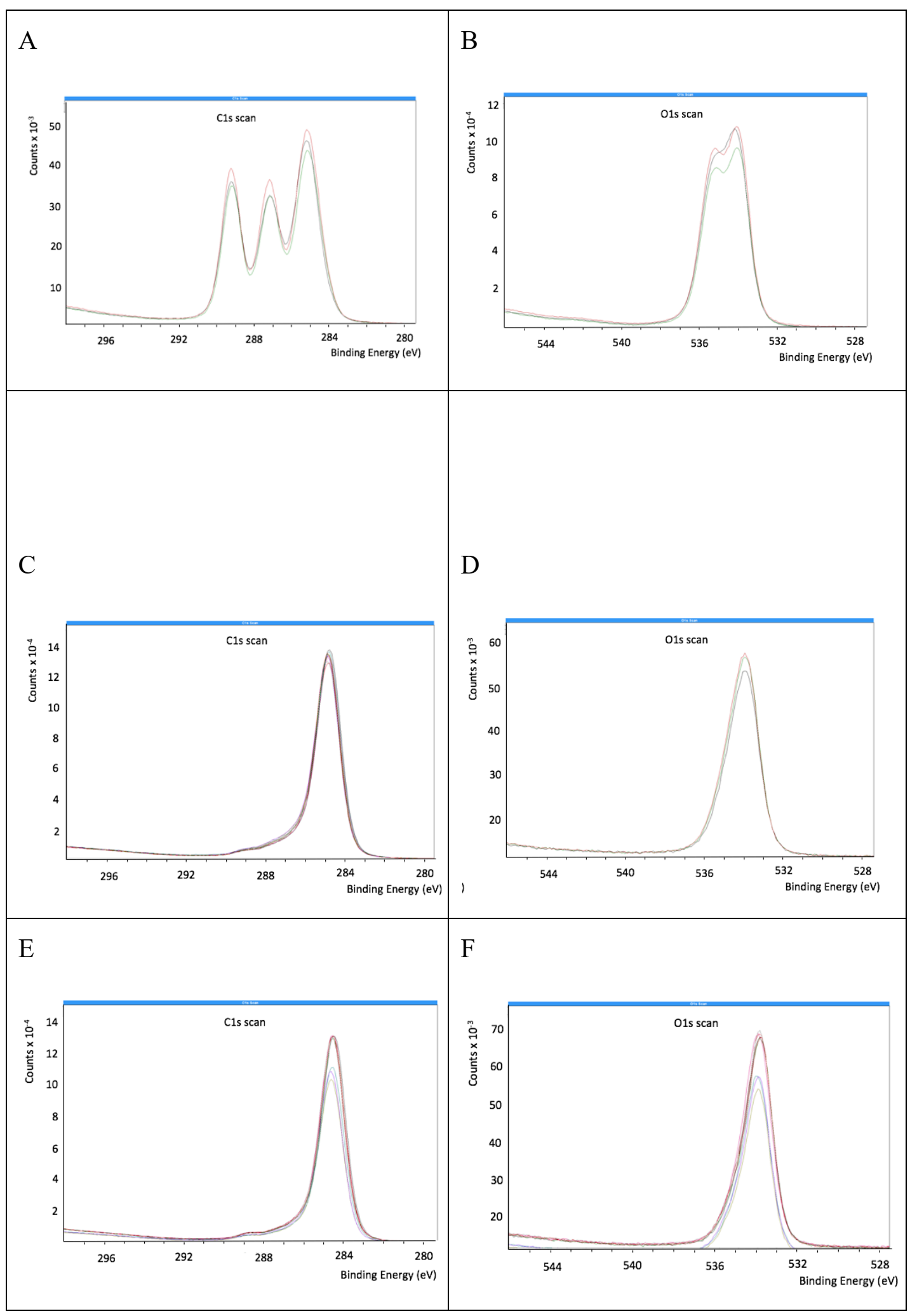


Figure 28. XPS analysis of PEA surfaces on PLA substrate. XPS spectra, around the bind energy for $\mathrm{C} 1 \mathrm{~s}$ (left column) and for O1s (right column) for spin coated amorphous PLA (A and B), and for the same spin coated amorphous PLA coated with plasma polymerised EA at $180 \mathrm{~kJ}(\mathrm{C}$ and D) and at $90 \mathrm{~kJ}(\mathrm{E}$ and $\mathrm{F})$.

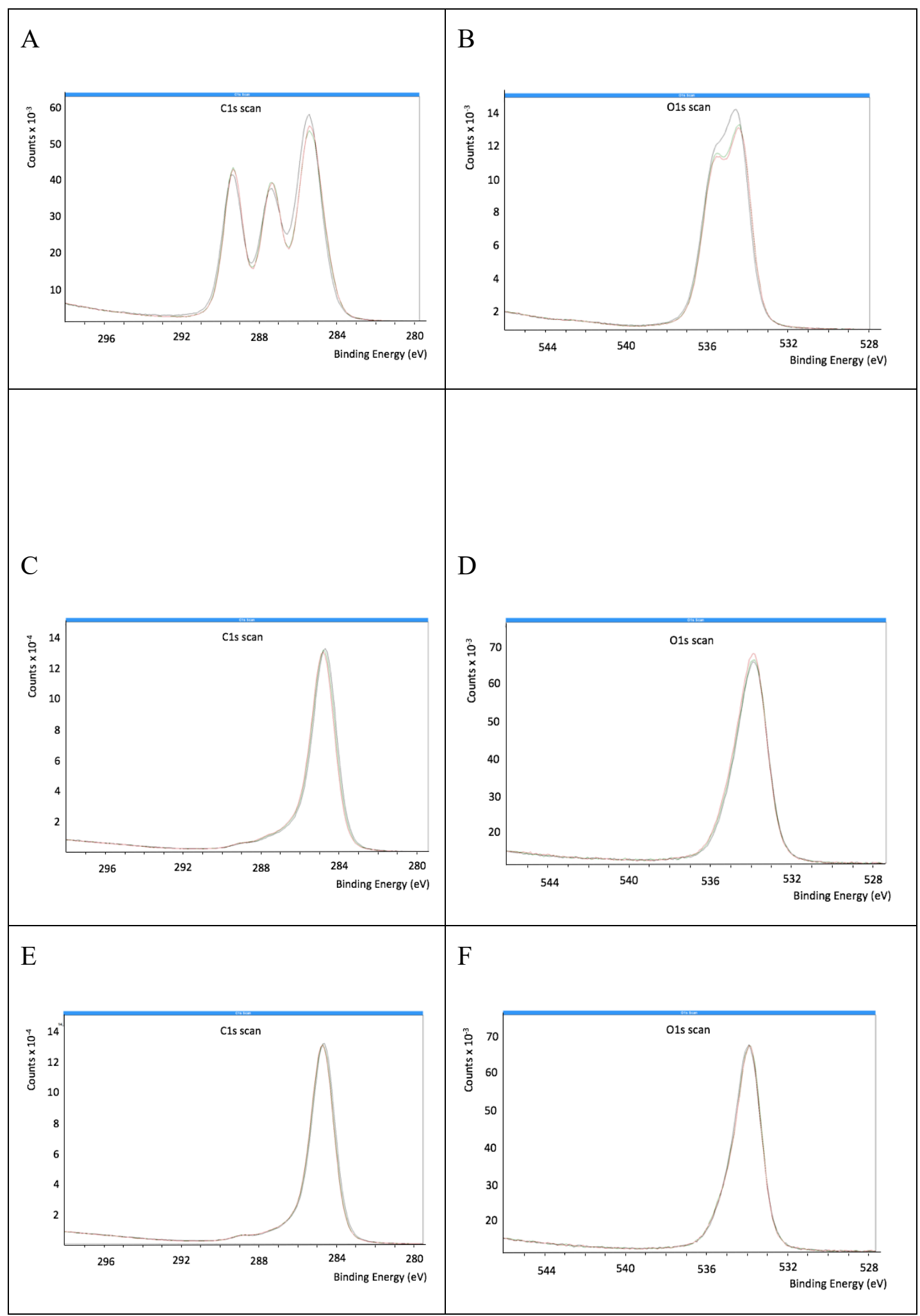


Figure 29. XPS analysis of PEA surfaces on PLA substrate. XPS spectra, around the bind energy for C1s (left column) and for O1s (right column) for spin coated crystalline PLA (A and B), and for same spin coated crystalline PLLA coated with plasma polymerised EA at $180 \mathrm{~kJ}(\mathrm{C}$ and $\mathrm{D})$ and at $90 \mathrm{~kJ}(\mathrm{E}$ and $\mathrm{F})$.

In summary, with the XPS analysis of the polymerized coatings we conclude that these coatings maintain some of the characteristic carbon and oxygen spectra of PEA. We can also conclude that lower plasma energies during the polymerization translate to increased retention of carbonyl and acid groups (small shoulder peaks at $289 \mathrm{eV}$ ) and of primary oxygen. Plasma polymerisation produces oxygen deficient PEA coatings, with the level of remaining oxygen-based functional groups directly depending on applied energy. Finally, we confirmed with an amourphous and crystalline (rougher) PLA substrate that the thickness of the deposited coatings guarantees that the XPS spectra is from the coating and not from the underlying substrate, as expected because the sensitivity of XPS is around $10 \mathrm{~nm}$. 


\subsection{Proteins on plasma coated surfaces}

\subsubsection{Conformation of adsorbed fibronectin}

In figures 30 to 37 below, we show plasma polymerised materials coated with FN. In this section we worked with a standard concentration of $20 \mu \mathrm{g} / \mathrm{ml}$ commonly used in the in vitro and in vivo experiments in our lab, and in the following section we studied $\mathrm{FN}$ conformation at lower concentrations (of $1 \mu \mathrm{g} / \mathrm{ml}$ and $500 \mathrm{ng} / \mathrm{ml}$ ).

Previous work in our group has shown that PEA triggers spontaneous organization of FN into nanonetworks which involves the simultaneous availability of the integrin binding region (FNIII 9 -10) and growth factor binding region (FNIII $12-14)$. In this work we set out to verify the morphology and conformation of the protein on the plasma polymerised materials, to find out the capability of pPEA to maintain or promote FN fibrillogenesis as seen with bulk PEA (spin coated PEA). As reviewed in section 1.1 this is a critically important functional aspect of these materials for their potential use in tissue engineering applications. To characterise FN absorption we have used AFM scans, water contact angle, fluorescence labelling for optical microscopy, and micro BCA, while FN domain availability and BMP-2 adsorption was characterised by ELISA. These experiments for protein adsorption have used four selected plasma conditions $(4.5,15,45$ and $180 \mathrm{~kJ})$. Figure 34D shows the WCA measured on the PEA surfaces, with and without FN coatings. All pPEA and SC-PEA coatings are highly hydrophobic surfaces with water contact angle of around $73^{\circ}-88^{\circ}$. However, all PEA surfaces show significantly different values to glass and became more hydrophilic once coated with FN. With FN coating the contact angle was observed within the range $40^{\circ}-55^{\circ}$ (Figure 37D). On uncoated control glass surfaces, there is not much difference in wettability with or without FN coating. With respect to surface wettability of biologically active materials, cells effectively adhere onto surfaces presenting moderate wettability with water contact angles of $40^{\circ}-70$.

However, the conformation and distribution of FN adsorption onto pPEA is different to SC-PEA surfaces (Figure 30). These control surfaces scanned with AFM show FN networks as previously reported in the literature by our lab on acrylate polymers. In contrast, AFM scans of surfaces coated with plasma polymerised materials (Figures 32 and 31) show a distinct and characteristic conformation of the protein, throughout all the different plasma deposition conditions, that doesn't resemble the control networks seen on figure 27 but that appears nevertheless to have a tight organization of the proteins. With a similar organization for all plasma polymerization conditions, on AFM scans threads of protein groupings or aligned protein strands, with small gaps within the structure, can be seen at the sub-micron scale. We see this conformation on plasma polymerized acrylate as a 'compact network' of FN. This compact network conformation has little or no apparent variation with the plasma polymerisation conditions, i.e. duration of monomer plasma stage and (10 to 30 minutes) or applied incident power (100 to $25 \mathrm{~W}$ ). This different organisation of FN is likely due to the 
differences in surface chemistry as well as the compositions of the polymers as shown by XPS analysis in particular.

Using fluorescence labelling for optical microscopy we have seen at the super micron scale that the protein coatings show heterogeneous features on both control spin coated EA surfaces and plasma polymerized EA surfaces (Figure 36). The protein on these surfaces seems to have a characteristic organization with random holes in size and distribution that could arise from a process of surface wettability, but that don't show the same on different samples or even in different areas of the same samples.

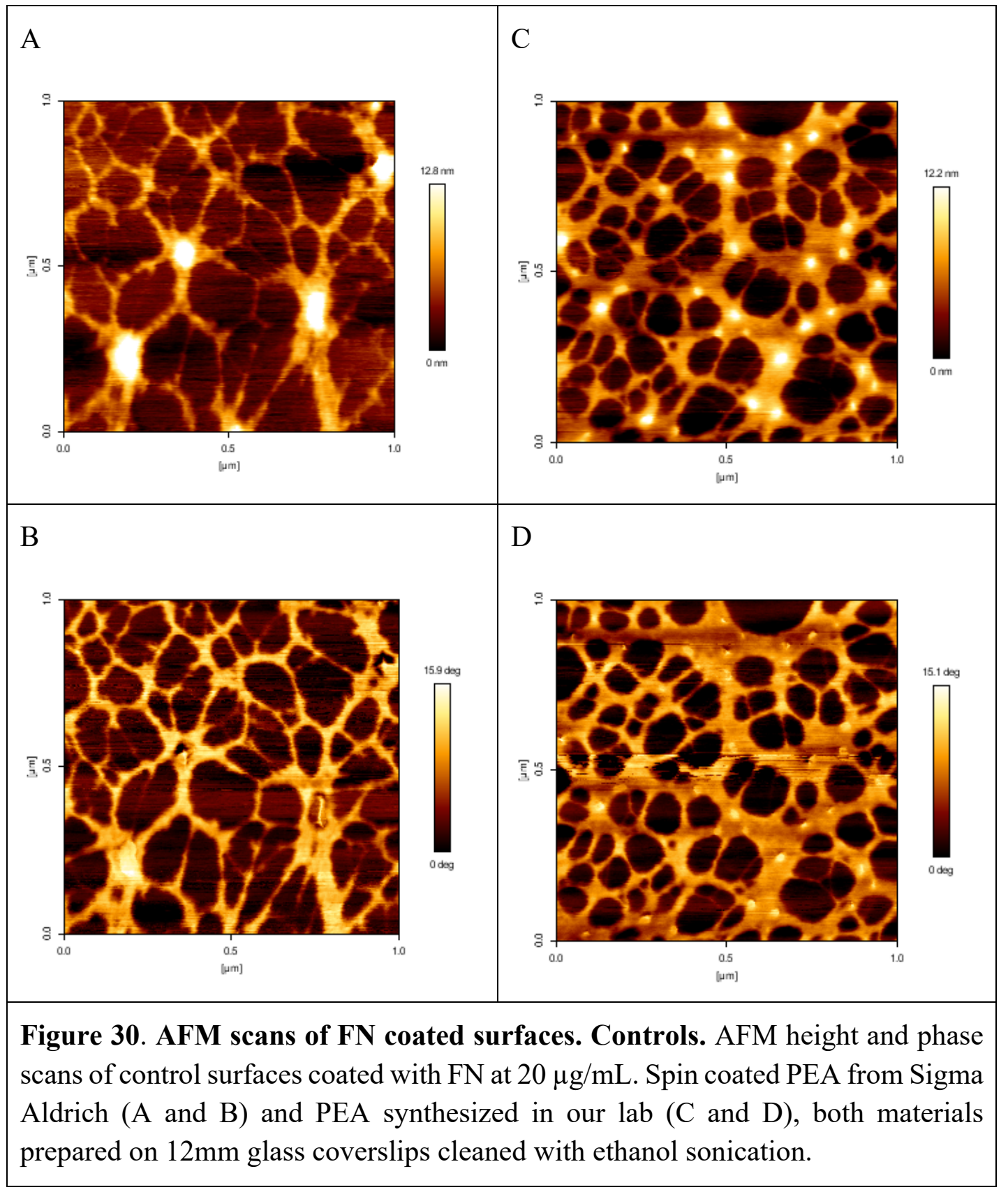




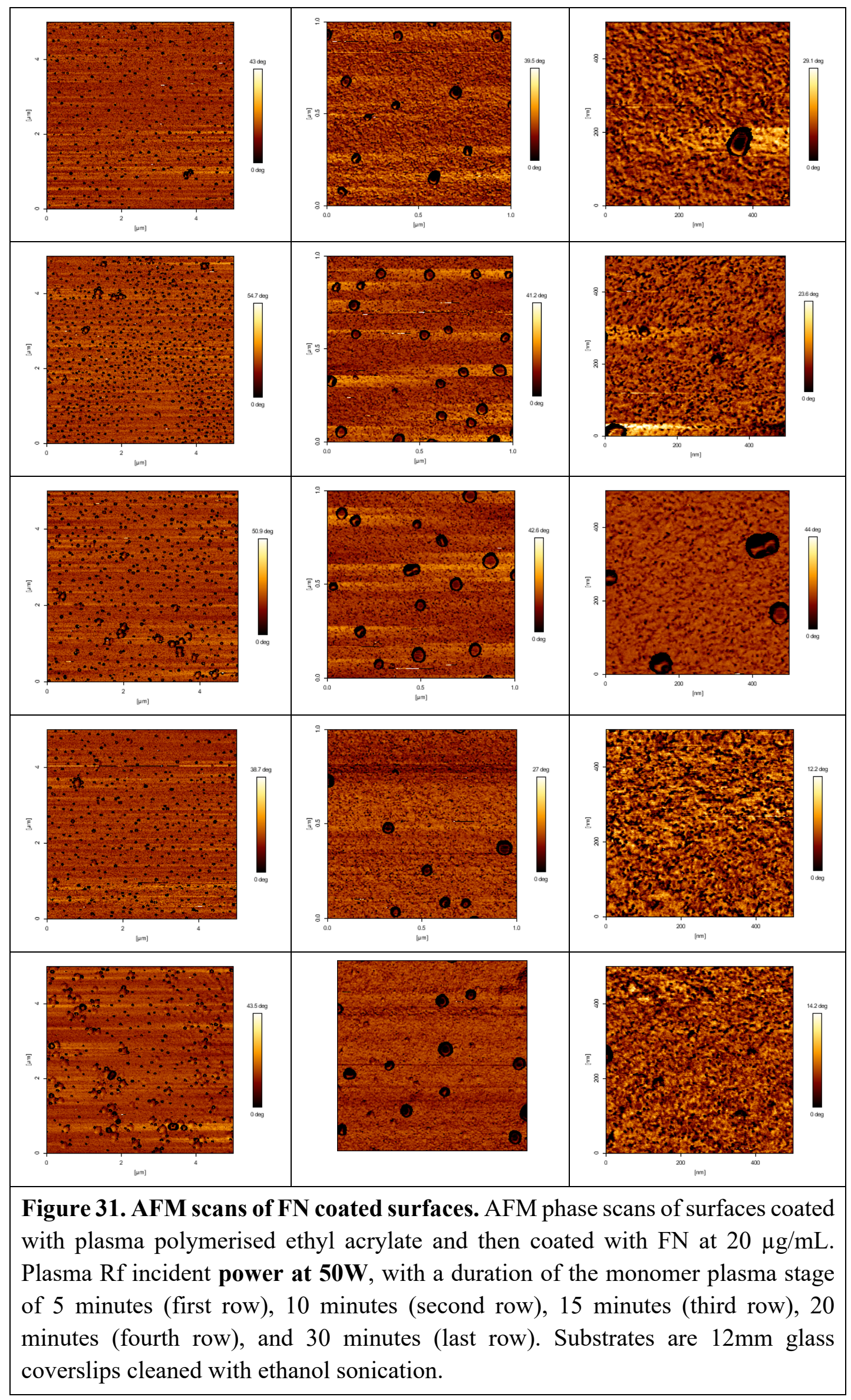



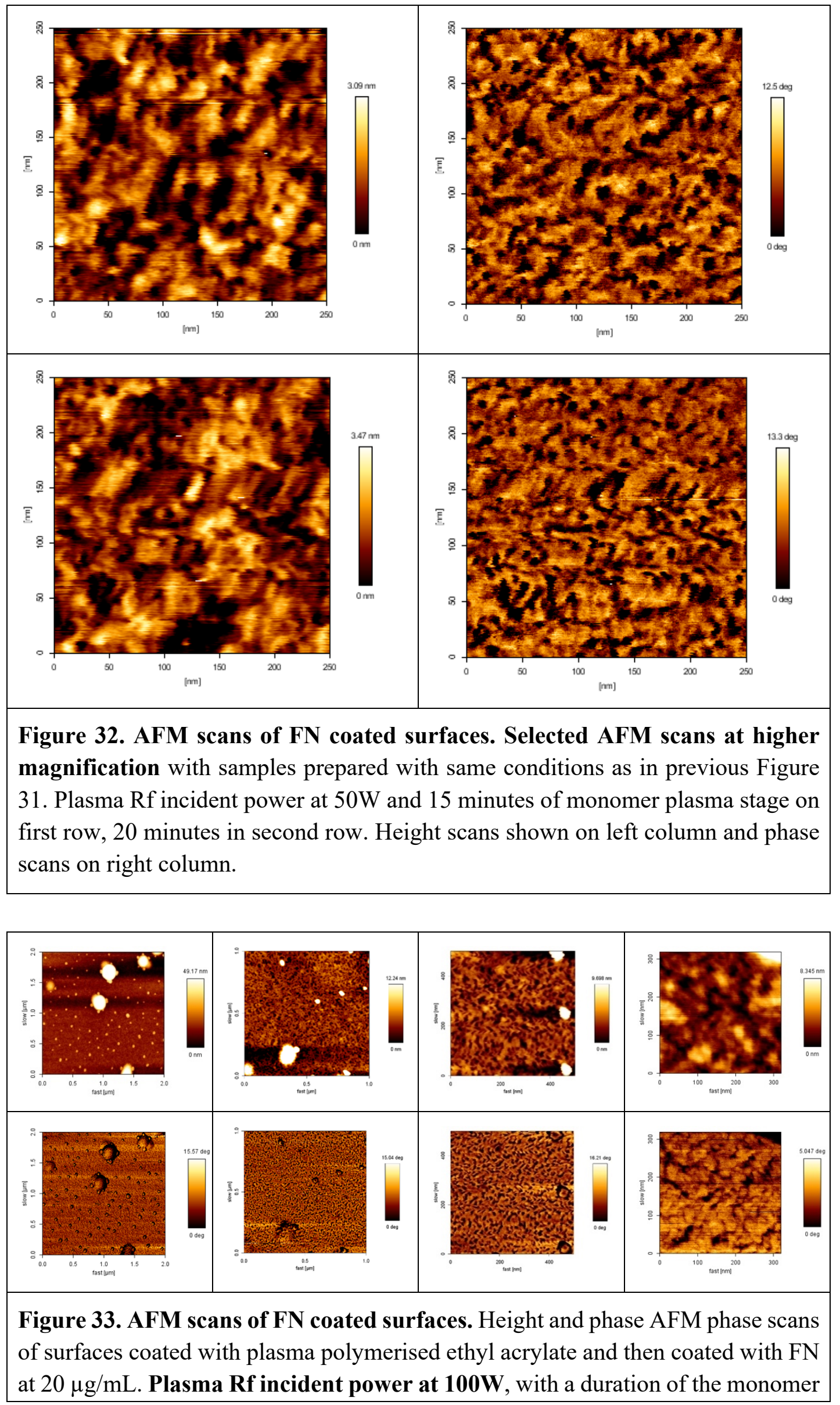
plasma stage of $\mathbf{3 0}$ minutes. Substrates are $12 \mathrm{~mm}$ glass coverslips cleaned with ethanol sonication.

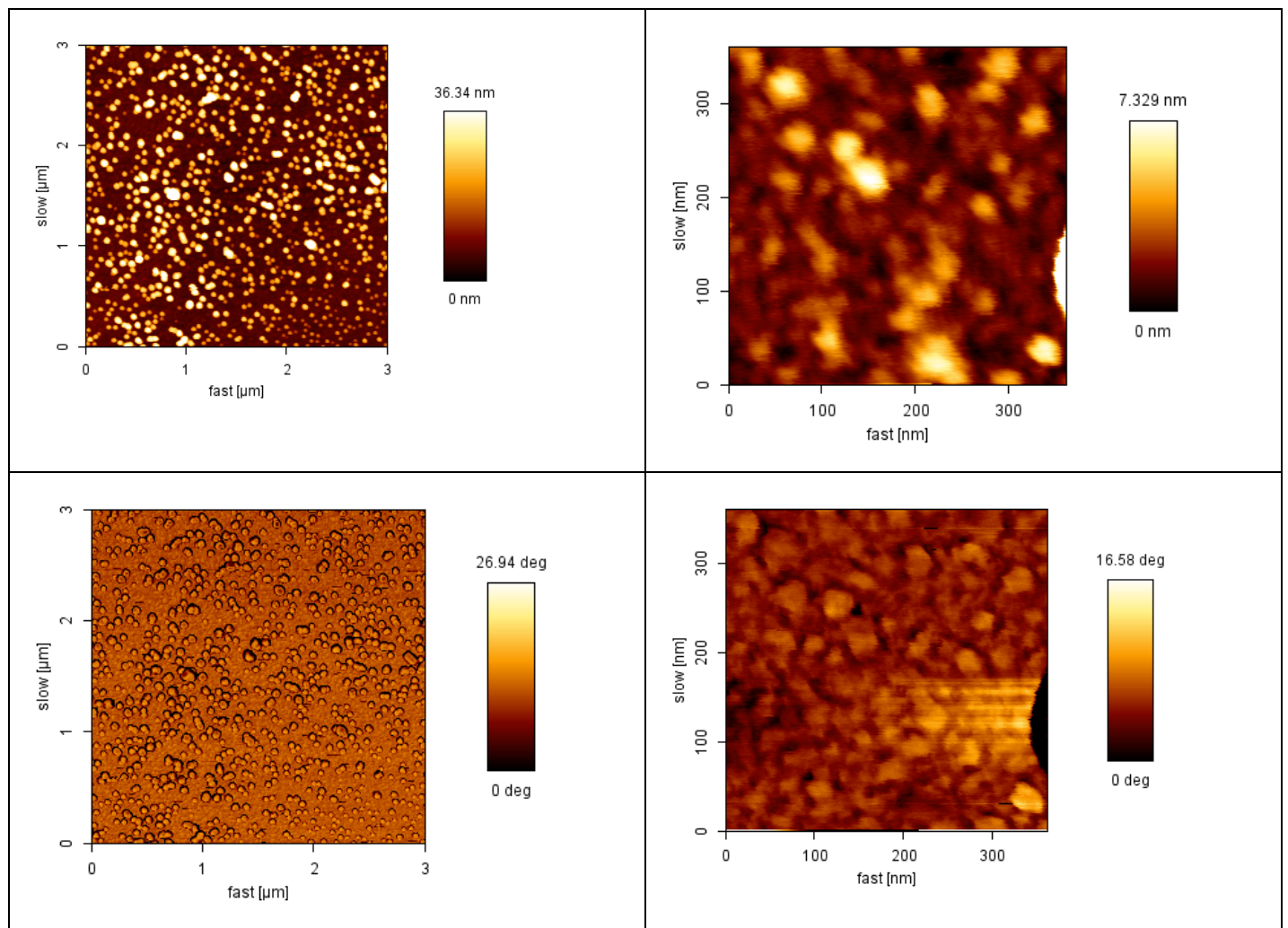

Figure 34. AFM scans of FN coated surfaces. Height and phase AFM phase scans of surfaces $(12 \mathrm{~mm}$ glass coverslips cleaned with ethanol sonication) coated with plasma polymerised ethyl acrylate and then coated with FN at $20 \mu \mathrm{g} / \mathrm{mL}$. Plasma Rf incident power at $100 \mathrm{~W}$, with a duration of the monomer plasma stage of $\mathbf{1 5}$ minutes.

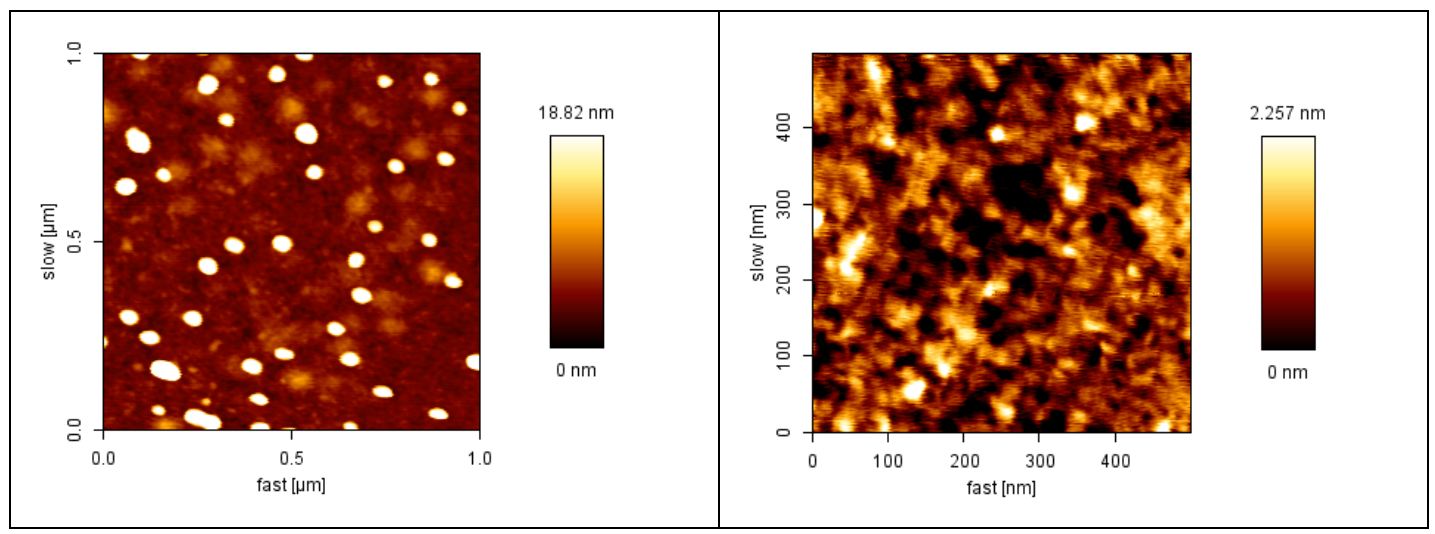




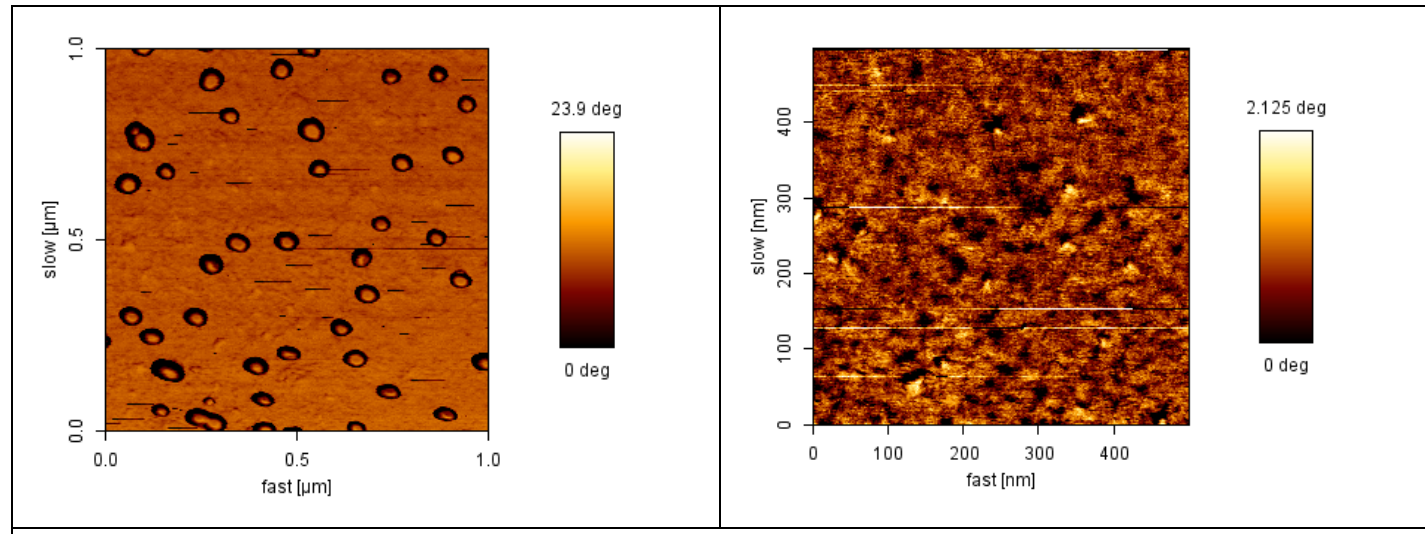

Figure 35. AFM scans of FN coated surfaces. Height and phase AFM phase scans of surfaces (12mm glass coverslips cleaned with ethanol sonication) coated with plasma polymerised ethyl acrylate and then coated with FN at $20 \mu \mathrm{g} / \mathrm{mL}$. Plasma Rf incident power at $\mathbf{2 5 W}$, with a duration of the monomer plasma stage of $\mathbf{1 5}$ minutes.

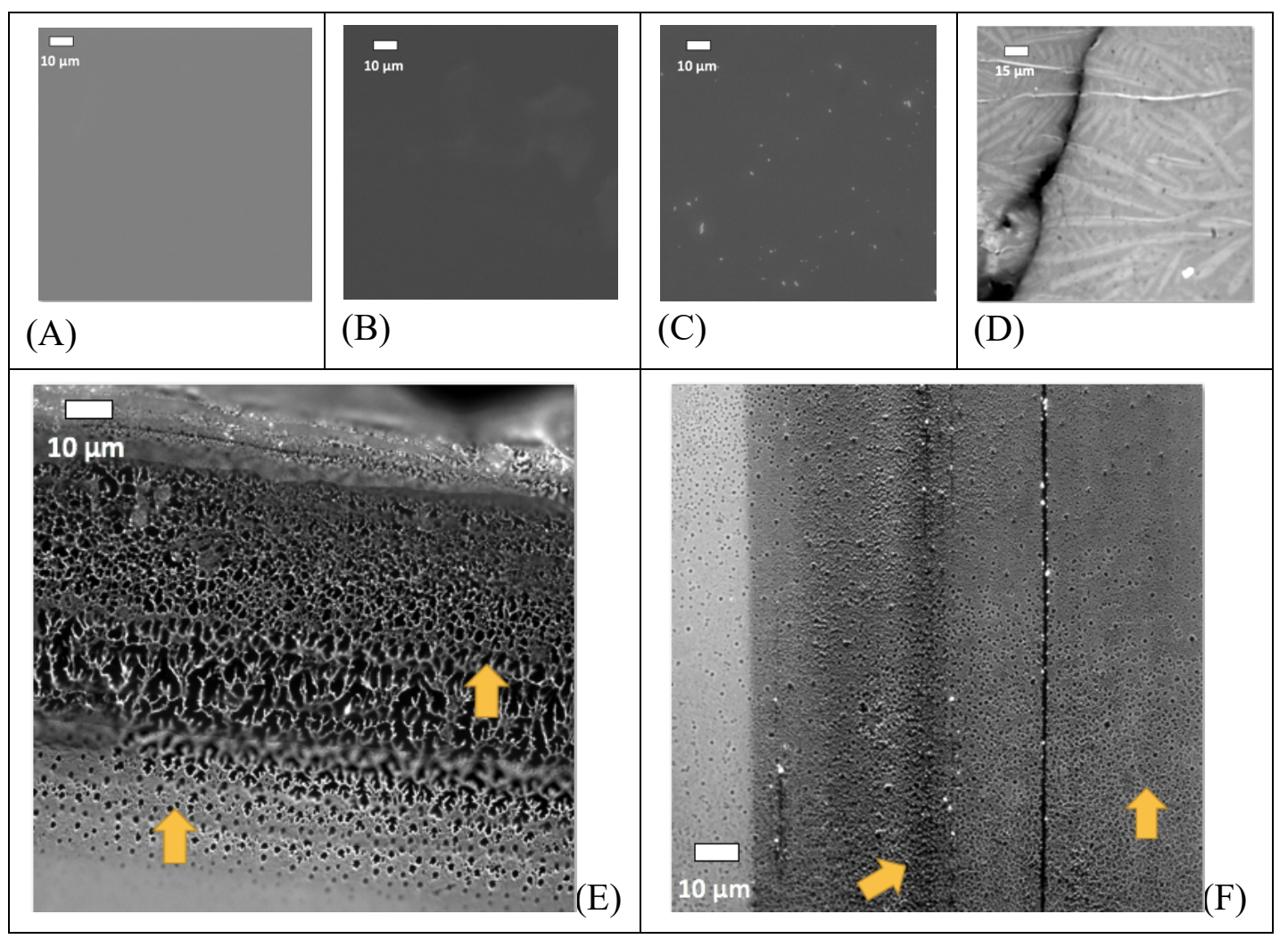




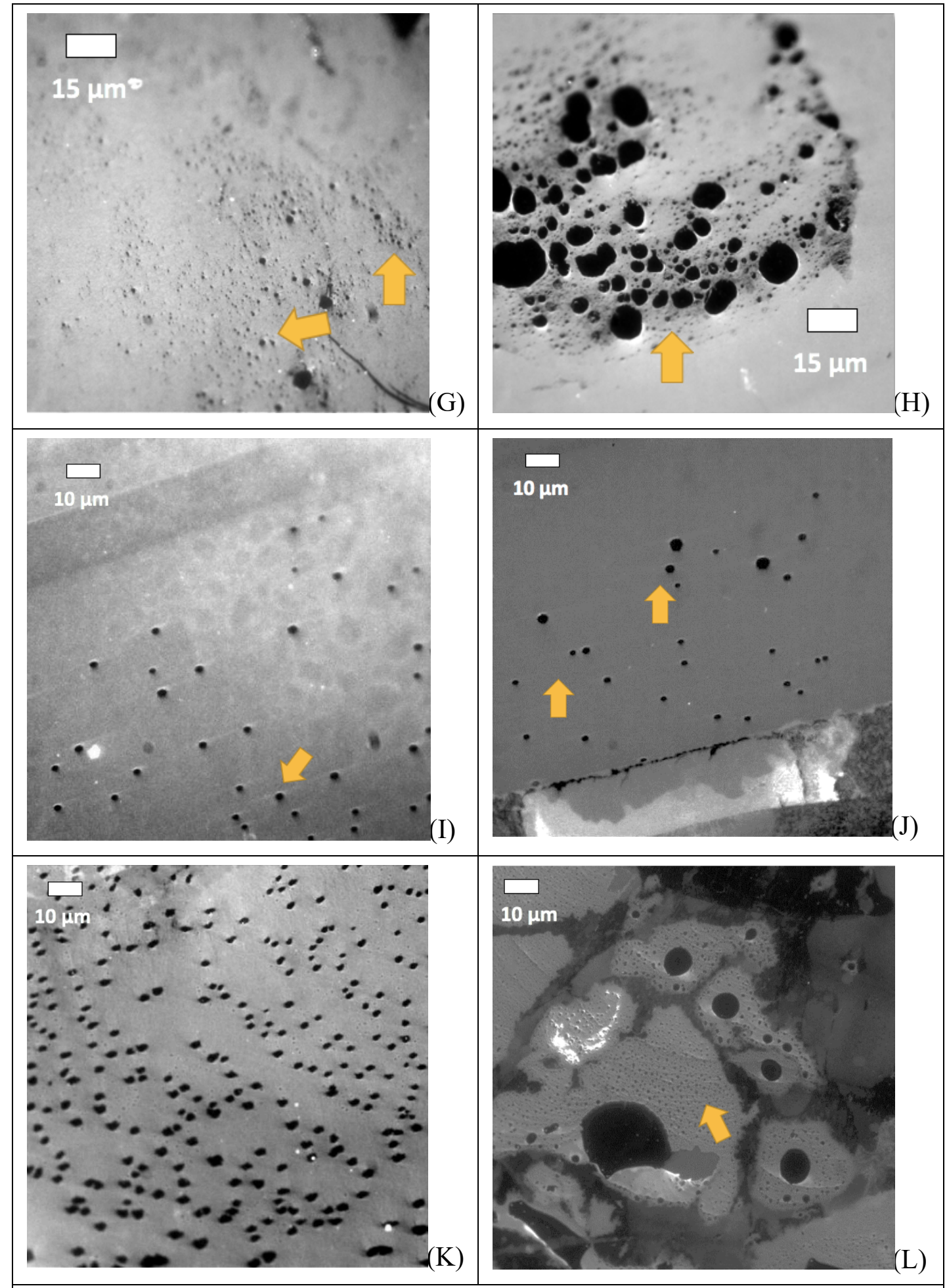

Figure 36. Fluorescence microscopy images of protein coated surfaces. All pictures at $63 \mathrm{x}$ magnification except (D) at 40x magnification. All coatings with FN at $20 \mu \mathrm{g} / \mathrm{mL}$. First row: (A) cleaned glass $12 \mathrm{~mm}$ coverslips, (B) spin coated PEA on glass, (C) plasma polymerized EA, Rf power at $50 \mathrm{~W}$, monomer plasma stage 15 minutes, (D) FN on glass. Second row: (E) and (F) FN on spin coated PEA. Third row: $(\mathrm{G})$ and $(\mathrm{H}) \mathrm{FN}$ on plasma EA 100W 30min. Fourth row: (I) and (J) FN on plasma EA 100W 15min. Last row: (K) and (L) FN on plasma EA $25 \mathrm{~W} 15 \mathrm{~min}$. 
The interaction of plasma polymerized PEA coatings with $\mathrm{FN}$ was further characterized quantifying surface densities of adsorbed FN on the different materials using bicinchoninic acid assay (BCA) and checking for FN domain availability with an enzyme-linked immunosorbent assay (ELISA). These experiments used four selected plasma conditions $(4.5,15,45$ and $180 \mathrm{~kJ})$. Adsorbed FN was quantified by measuring the amount of FN remaining in the supernatant after adsorption. Although the surface density of FN on all plasma polymerized PEA was slightly higher than that on SC-PEA and on glass surfaces, the difference was not significant (Fig. 37A). To evaluate the availability of integrin binding and growth factor binding domains on PEA surfaces after FN adsorption, we used an ELISA using two monoclonal antibodies. HFN7.1 is directed against the flexible link between $\mathrm{III}_{9}$ and $\mathrm{III}_{10}$ in FNIII9-10 repeat of FN, which is involved in integrin binding and cell adhesion (Fig. 37B). P5F3 is directed against the FNIII $12-14$ repeat which contributes to growth factor binding (Fig. 37C).

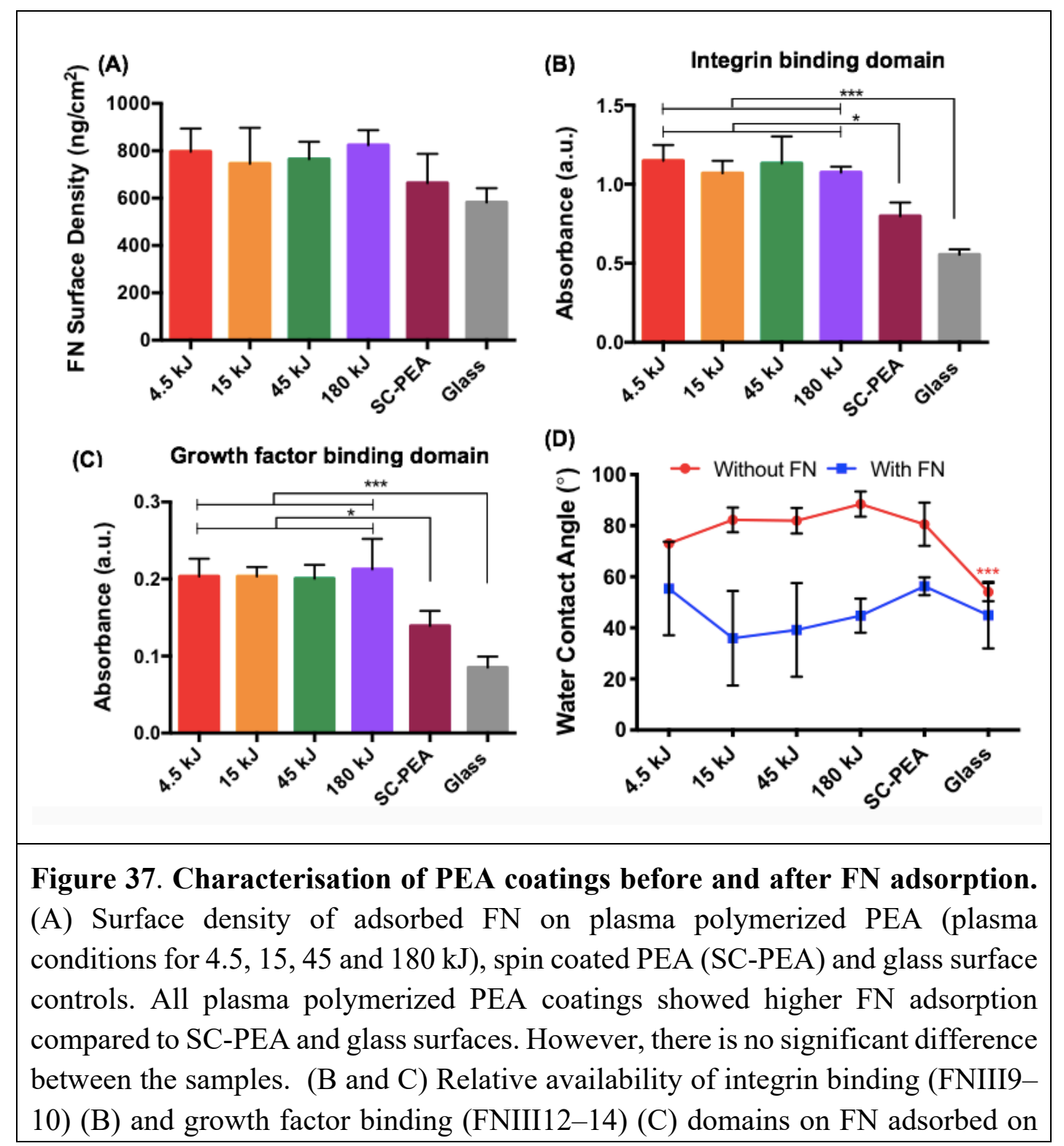


different surfaces, measured using ELISA. (D) Static water contact angle measurements of pPEA coatings, SC-PEA and glass surface with and without FN coating. Surfaces became more hydrophilic after absorption of FN.

Despite the surface density of FN on PEA and glass surfaces not being significantly different, we observed significantly higher availability of integrin and GF binding domains on pPEA in comparison to SC-PEA and glass surfaces. When these results were normalised to FN surface density, significant differences remained, indicating that pPEA coatings induces an increased level of FN fibrillogenesis.

These results suggest that FN assemble on pPEA surfaces into a dense network of nanofibrils that is functionally active to present simultaneously integrin binding and GF binding regions. We show that this property of pPEA is maintained regardless the condition used (energy in the plasma reactor) during plasma polymerisation.

\subsubsection{Conformation of adsorbed fibronectin at low concentrations}

In order to figure out if the FN molecules organize in networks or in any other manner that implies some kind of fibrillogenesis, on the plasma polymerized EA surfaces, we used concentrations of $1 \mu \mathrm{g} / \mathrm{ml}$ and $500 \mathrm{ng} / \mathrm{ml}$. Following the line of work of the previous section we produced plasma polymerised materials coated in our experiments with FN at these concentrations much lower than the usual $20 \mu \mathrm{g} / \mathrm{ml}$. We speculated that on the surfaces coated with the plasma polymerized EA the FN is adsorbed in a more compact manner than on the surfaces coated with acrylates produced by radical polymerization, and that a lower FN concentration would allow us to have more information about the morphology and conformation of FN on these surfaces.

In figures 38 and 39 below we show AFM scans of plasma polymerised materials coated with FN, in this section at the aforementioned low concentrations of $1 \mu \mathrm{g} / \mathrm{ml}$ and $500 \mathrm{ng} / \mathrm{ml}$. In these samples the FN coating was prepared in the same manner as in the previous section. Even at these low concentrations we see the protein fully covering the surface and organized in 'clumps' that appear to be elongated and aligned in the same direction. At the highest magnifications we see that these clumps of protein seem to be made of several smaller clumps. 


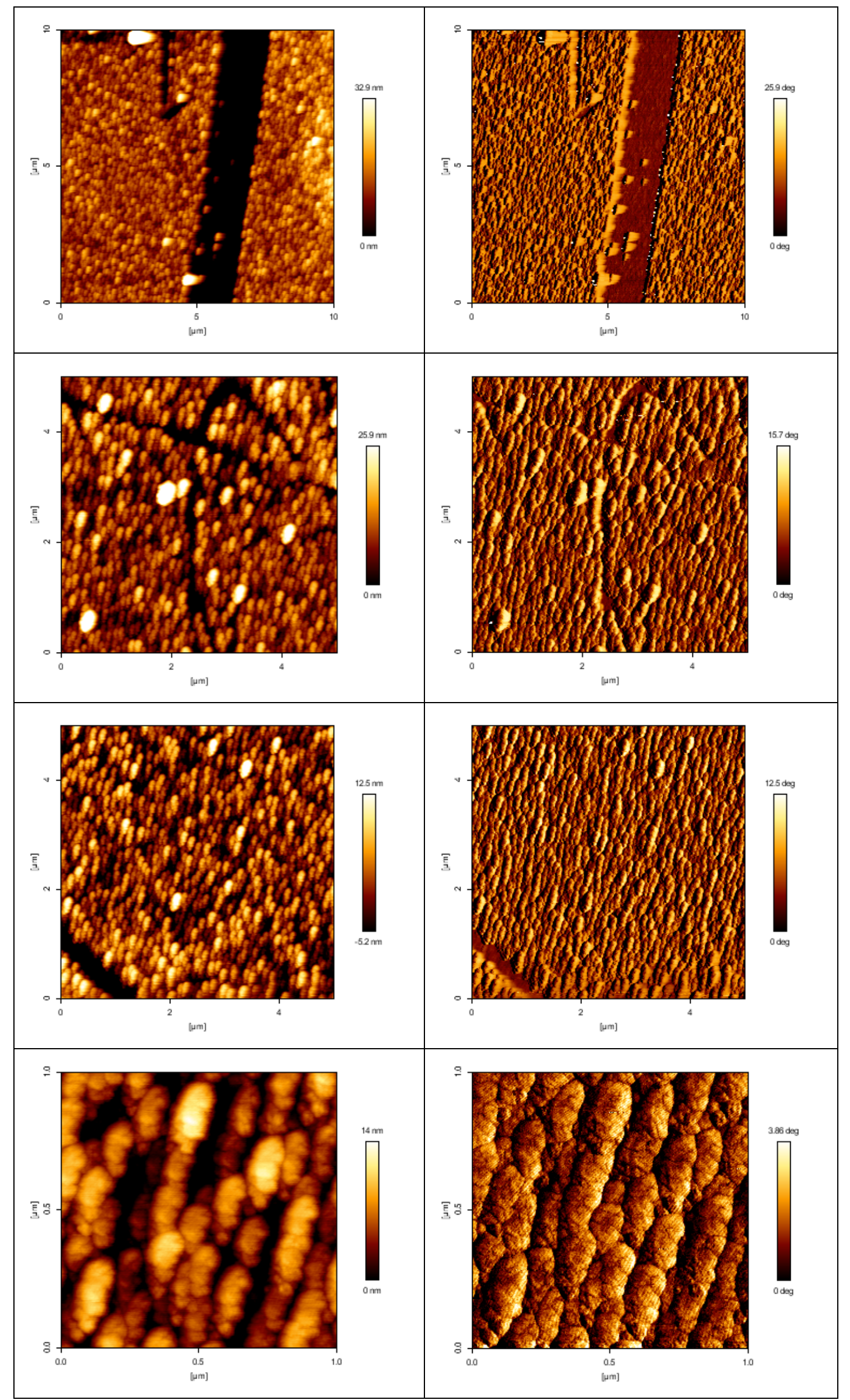




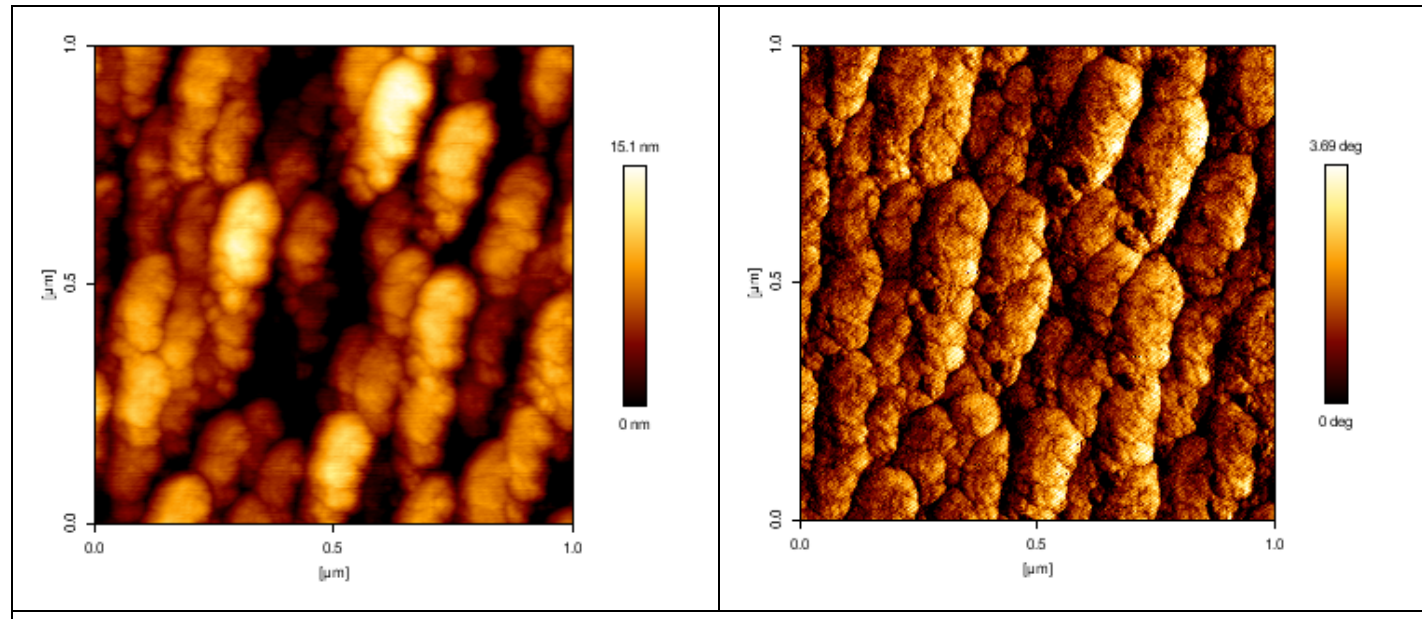

Figure 38. Conformation of $\mathrm{FN}$ at low concentration on plasma polymerised surfaces. Height (left column) and phase (right column) AFM scans of glass surfaces coated with plasma polymerised PEA and low concentration FN. All samples prepared with plasma Rf incident power at $100 \mathrm{~W}$, with a duration of the monomer plasma stage of 15 minutes, and then coated with FN at $1 \boldsymbol{\mu g} / \mathbf{m l}$. First row shows $10 \mu \mathrm{m}$ by $10 \mu \mathrm{m}$ scans with markings/scratching on the protein surface made with a pipette tip.

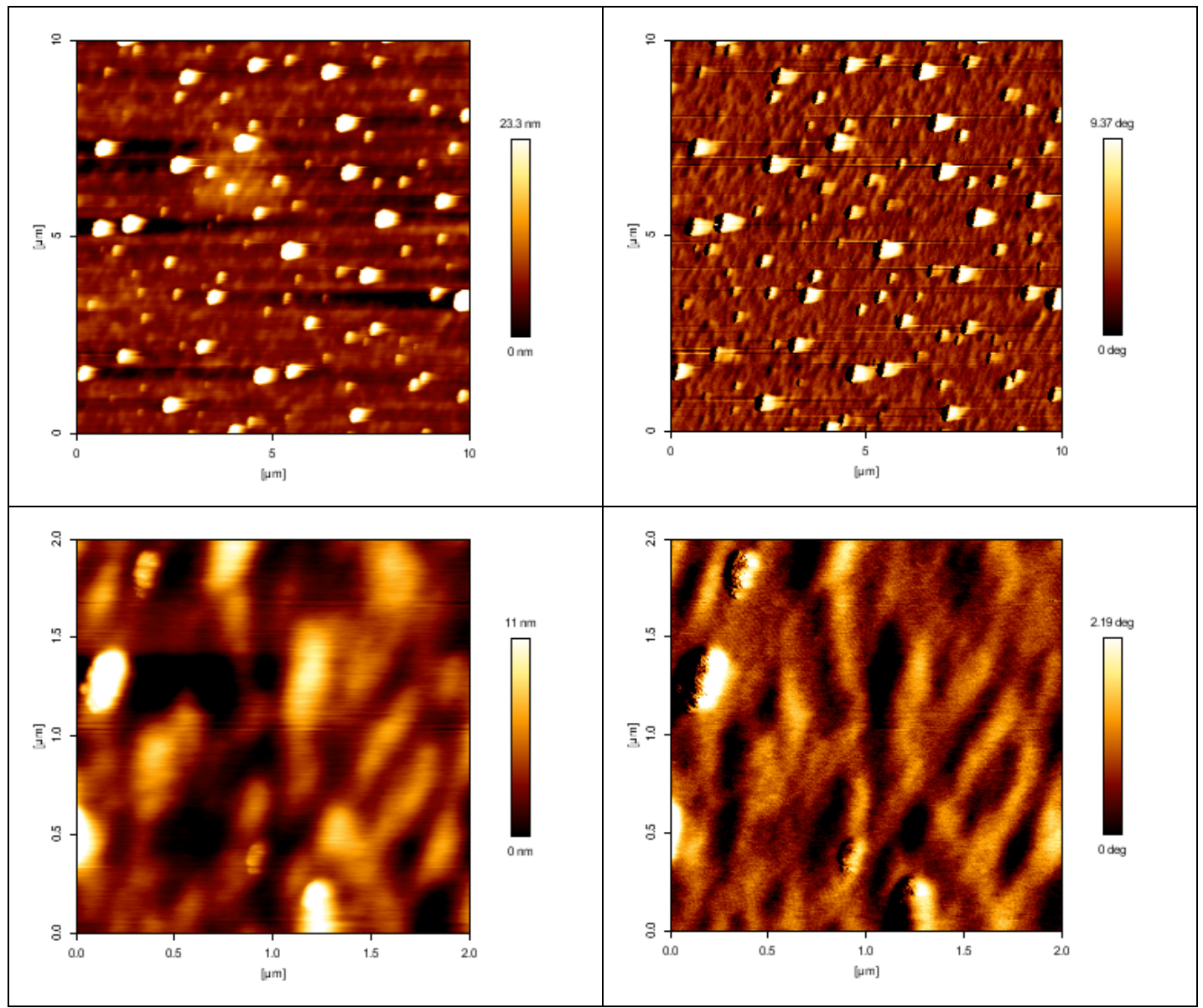




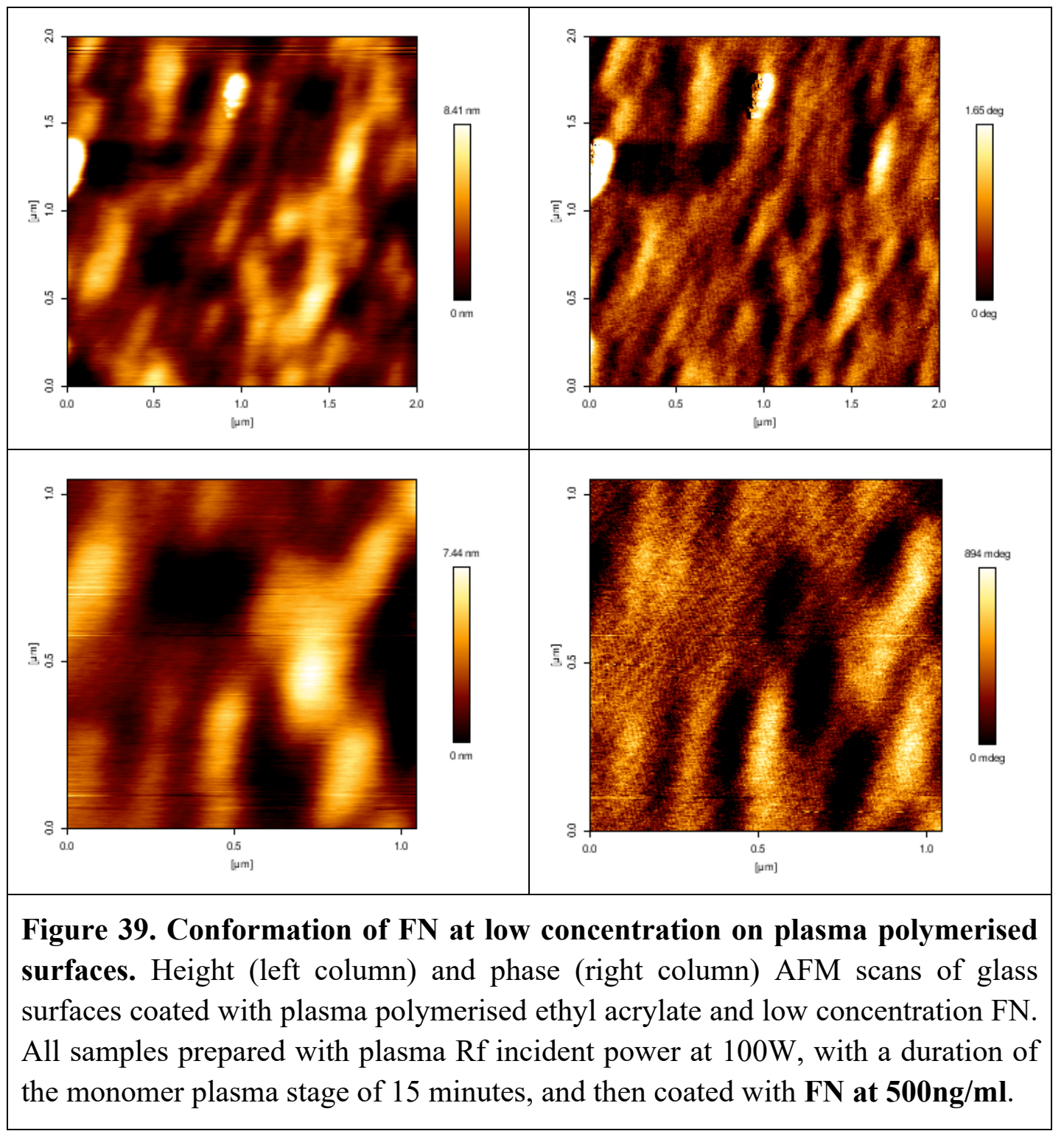

\subsubsection{BMP-2 adsorption}

We also performed ELISA assays to assess whether the differential conformation of FN affects the surface density of BMP-2 bound on FN coated surfaces. We used a solution at a concentration of $50 \mathrm{ng} / \mathrm{ml}$ BMP-2 to coat six different surfaces. Glass and spin coated PEA (SC-PEA) as negative and positive controls respectively, and four plasma polymerised coatings at plasma conditions for 4.5, 15, 45 and $180 \mathrm{~kJ}$. After coating with FN, and BMP-2, we have seen BMP-2 adsorption to be significantly higher on plasma polymerized surfaces than on SC-PEA and glass surfaces (figure 40). 


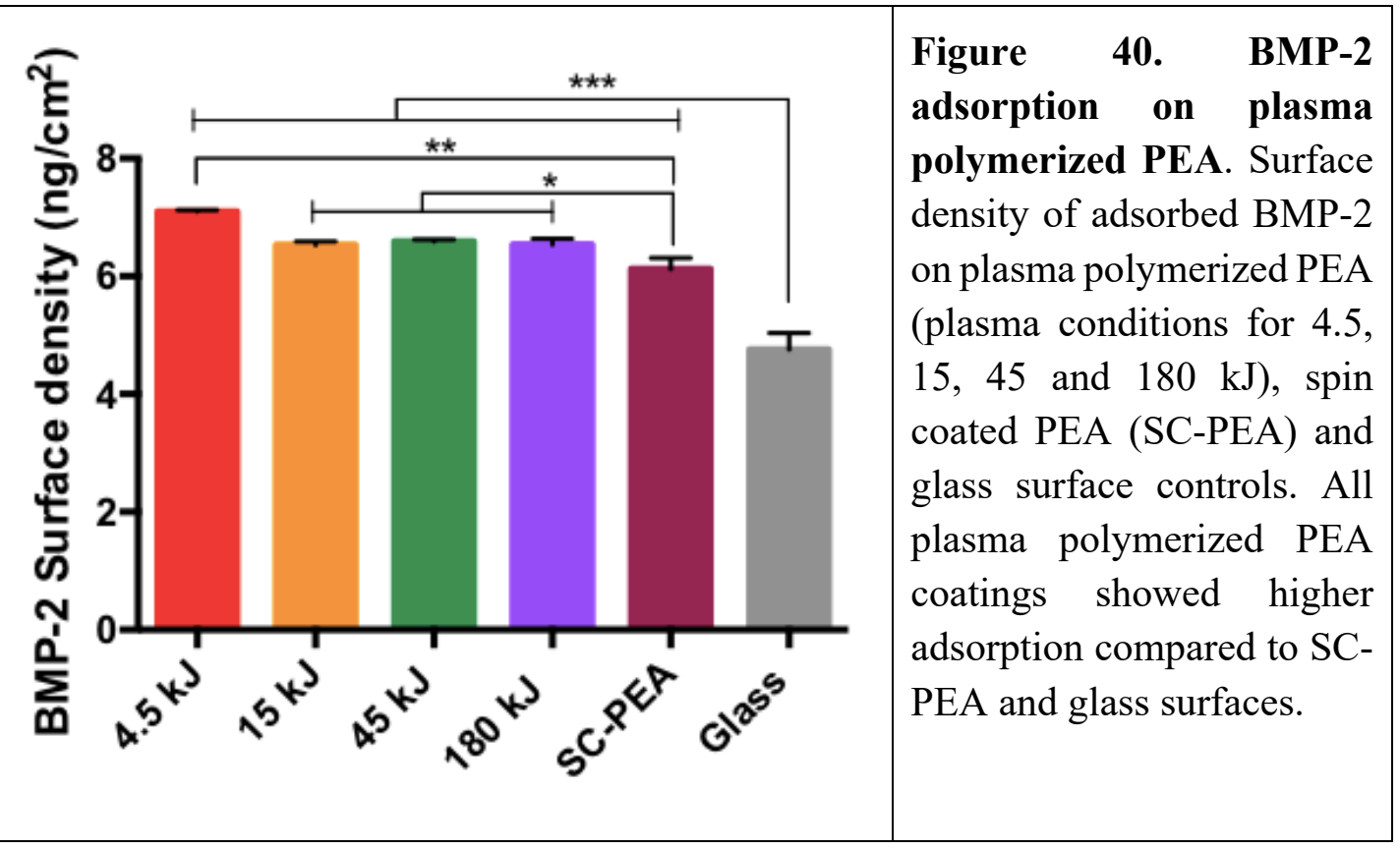

This result supports the data on GF binding domain availability discussed in section 4.3.1. More BMP-2 adsorbed on FN coated on plasma polymerized PEA than on SCPEA and glass surfaces.

Together with the previous results on FN adsorption and FN integrin binding domain availability, these results suggest that FN assemble on plasma polymerized PEA surfaces into a dense network of nanofibrils that is functionally active to present simultaneously integrin binding and GF binding regions. We show that this property of plasma polymerized PEA is maintained regardless the condition used (energy in the plasma reactor) during plasma polymerisation. 


\subsection{Coating of scaffolds}

Scaffolds based on synthetic and natural materials are used and studied as clinical strategies to heal large bone defects and non-unions [56]. Coating the scaffolds with biotherapeutics is a known strategy to develop therapies that improve bioactivity and osteoinductivity of the substrate materials. As part of our work developing and characterizing the PEA plasma polymerized coatings, we studied its application to 3D structures, in particular scaffolds that could be used in the orthopaedic treatment of large bone defects and non-union fractures.

We developed protocols to coat three types of scaffolds as part of other related research work in the lab. We coated microporous PLA scaffolds manufactured by ourselves with a combination of freeze extraction and porogen leaching techniques, as detailed in the M\&M section. We also coated PCL scaffolds fabricated with an extrusion-based 3D printer. Both the PLA and PCL scaffolds were manufactured with medical grade polymers, to develop the manufacturing and coating protocols as ready as possible for clinical use (figure 41). In appendix 7.3 we add more information about the developed coating protocols.

Finally, we also coated commercial dog allogeneic bone chips. These bone chips were used for a in a world-first intervention on a dog, a first veterinary patient treated with this bone regeneration technology [7] [46] (figure 42).

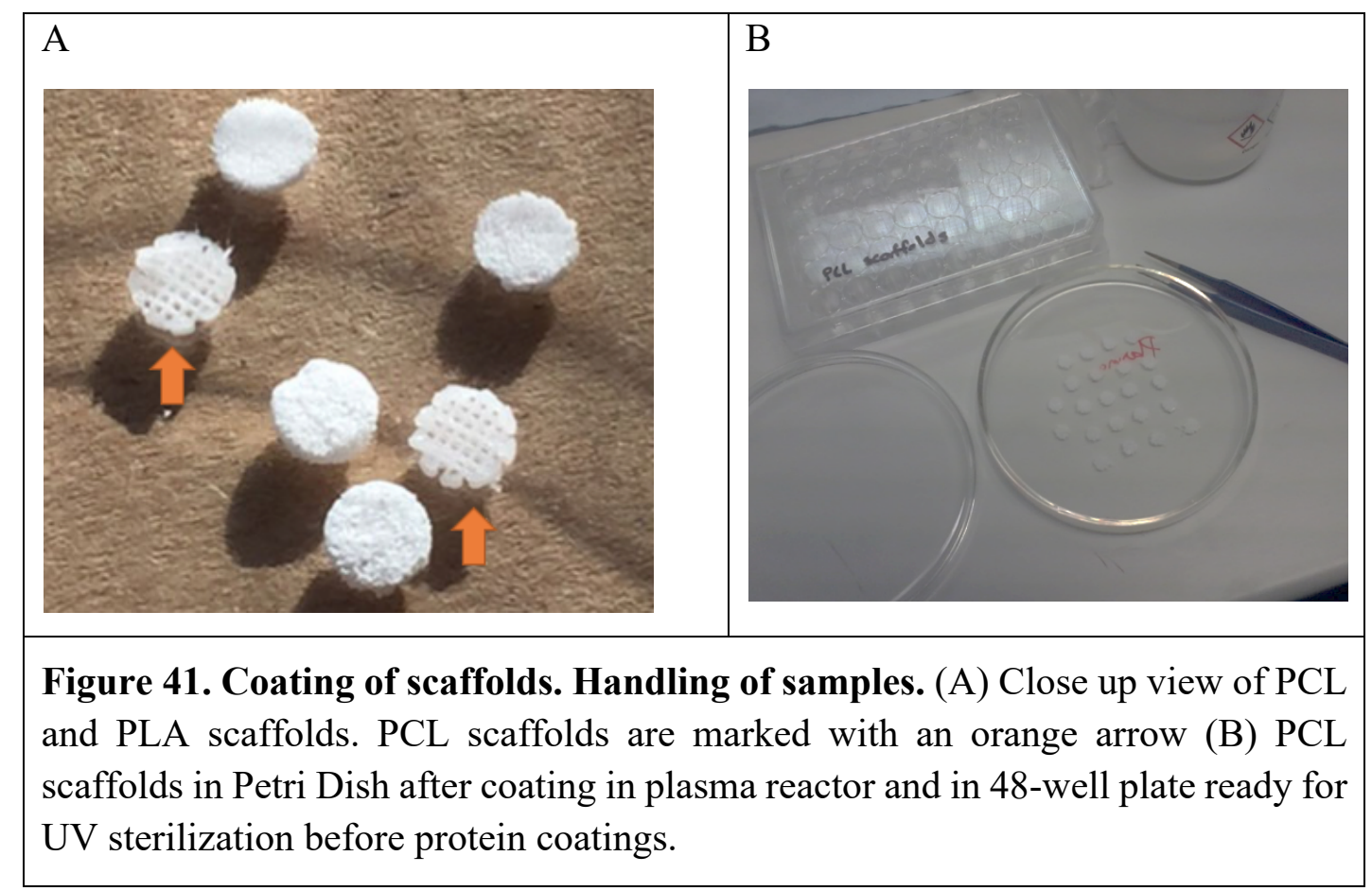

\subsubsection{Coating scaffolds with proteins}

For the veterinary trial we coated commercial dog allogeneic bone chips with plasma polymerized PEA and also helped develop the coating protocol for fibronectin and growth factor to be adsorbed onto the polymer coating. For preliminary tests we used 
PLA scaffolds and food colouring to confirm that the protein would reach all the surface areas inside the scaffold. We cut scaffolds from $1 \mathrm{~mm}$ to $4 \mathrm{~mm}$ of thickness and immersed them in cell culture media with added food colouring, to verify visually if the media reached the inner surfaces of the scaffold. With these scaffolds we confirmed that soaking the scaffolds under vacuum is an efficient and quick method to coat all the inner pores of its structure with protein (figure 42), while just leaving the scaffolds immersed in media results in the protein not reaching all areas of the scaffold. The method of immersing the scaffolds in the protein solution for a few minutes under vacuum was the method used for the allogeneic bone chips for the veterinary trial.

\begin{tabular}{|c|c|}
\hline $\mathrm{A}$ & 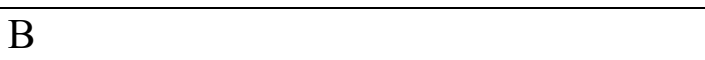 \\
\hline $\mathrm{C}$ & $\mathrm{D}$ \\
\hline $\mathrm{E}$ & $\mathrm{F}$ \\
\hline \multicolumn{2}{|c|}{$\begin{array}{l}\text { Figure 42. Coating of scaffolds. Tests with PLS scaffolds and coating of bone } \\
\text { chips for dog surgery. (A, B, C and D) Scaffolds coated in a DPBS solution with } \\
3 \% \text { paprika food colouring as a proof of concept for the protein coatings. Three } \\
\text { different thickness of scaffold tested: } 1 \mathrm{~mm}, 2 \mathrm{~mm}, 4 \mathrm{~mm} \text {, all same diameter. (A and } \\
\text { B) Coating by } 1 \mathrm{hr} \text { adsorption in well-plate with DPBS (semi-floating), no other } \\
\text { manipulation. (A) Scaffolds after immersion. (B) Scaffolds ( } 2 \mathrm{~mm} \text { and } 4 \mathrm{~mm} \text { only) } \\
\text { cut with a surgical blade, exposing inner area. (C and D) Coating by } 6 \text { minutes under } \\
\text { vacuum or until boiling starts, placing samples with DPBS solution in flacon tubes } \\
\text { inside a lab desiccator. Then an additional } 45 \text { minutes in well-plate with the } \\
\text { solution. (C) Scaffolds after immersion. (D) Scaffolds ( } 2 \mathrm{~mm} \text { and } 4 \mathrm{~mm} \text { only) cut } \\
\text { with a surgical blade, exposing inner area. (E) Commercial dog allogeneic chips out }\end{array}$} \\
\hline
\end{tabular}


of last step of coating, after coating in plasma reactor and coating with FN and BMP-2, shown as delivered in surgery theatre. (F) Coated chips and cancellous graft, before mixing, as prepared by surgeon. 


\section{Results and discussion. Spray coatings.}

\subsection{Setting up a custom-made spray installation}

Spraying a mixture of soluble material and volatile solvent with pressurized air is a well-developed technique [57] to coat a huge variety of different substrates with functional layers, with low cost and large area production.

We built in our lab two custom-made installations to deposit PEA from solutions in toluene on surfaces and scaffolds. A first pilot installation was for a simple highvolume airgun. A second installation that allowed more precise control of the coating was for a low volume airbrush.

We tested and characterised the performance of both systems, i.e. effect on substrate of solvent reaching the target surface, morphology of sprayed surface, and thickness of coatings, with experimental parameters, i.e. concentration of PEA in toluene including tests of dilution with ethanol, duration of spraying, spraying pressure, and distance from nozzle to substrate surface. Air pressure, within the available practical range in our lab (from about 0.7 bar to 1.5 bar), was found to have a limited effect on coating rate and no noticeable effect on the size of droplets on partially coated surfaces.

We have used our spray coating systems to coat flat surfaces of interest, including glass, spin coated polymers, polymer blocks with rough surfaces, and also porous scaffolds. Results with samples of some of these surfaces are described here, while others have been prepared in parallel for other research projects, that have tested these and similar coatings in related biomedical applications. We optimised protocols and requirements to coat surfaces and scaffolds for the two spraying installations developed.

Our first spraying set-up was assembled around a cheap and very simple to use air gun (Figure 43) that was positioned inside a custom-built cage with pre-fixed positions at $400 \mathrm{~mm}, 500 \mathrm{~mm} \& 700 \mathrm{~mm}$ from nozzle to target surface. The cage was fully closed except for a side port to reach to the gun trigger. Pressurized air was supplied by a lab compressor and all experiments were performed at 0.7 to 1.1 bar of air pressure. The cage, with the air gun and the sample target inside, was positioned in a laminar flow hood in the lab. 

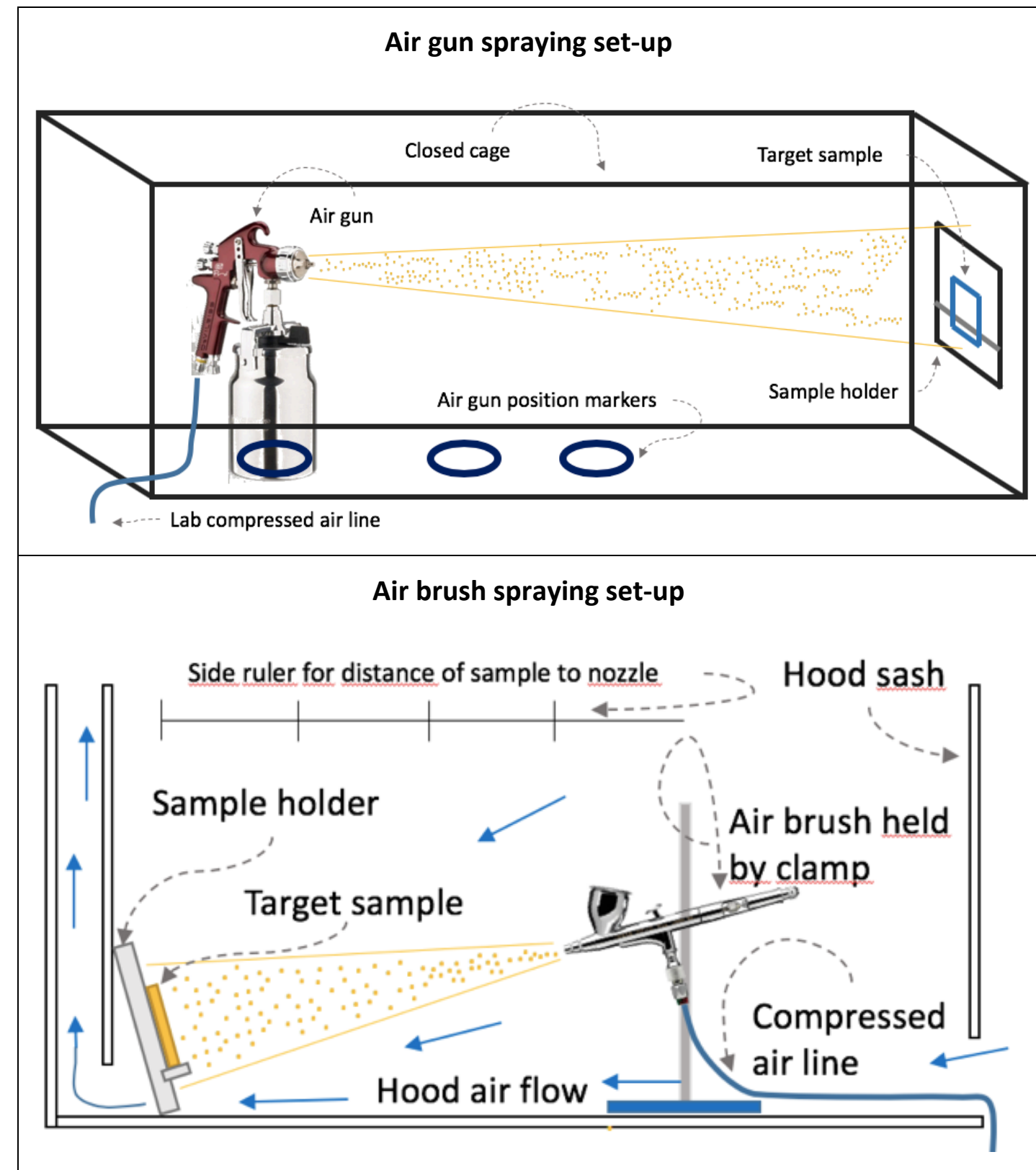

Figure 43. Schematics of the spraying set-ups. (Top) Air gun spraying set-up, inside a custom-built cage with pre-fixed positions at $400 \mathrm{~mm}, 500 \mathrm{~mm} \& 700 \mathrm{~mm}$ from nozzle to target surface. (Bottom) Air brush spraying set-up, with a dual action low volume air brush. Positioned with a lab clamp with a tilt to the horizontal and at the desired distance to sample target, with a maximum of $400 \mathrm{~mm}$.

Our second spraying set-up relied on a dual action low volume air brush that held in place with a retort steel base and was positioned with clamps to spray with a tilt to the horizontal and at the desired distance to sample target, with a maximum of $40 \mathrm{~cm}$ (Figure 43). We found that, for distances over $30 \mathrm{~cm}$, barely any material is deposited at our range of spraying conditions. The air brush that we acquired allowed certain control of the dispersion and shape of the spraying cone. The liquid container of the air brush is small, but one loading permitted all coating experiments even at the longest durations planned. The air brush was fully cleaned with pure toluene and 
ethanol after every experiment to avoid clogging of the delicate nozzle system. Experiments were also carried out inside a laminar flow hood in the lab.

\subsection{Air gun sprayed coatings}

With our air gun installation, we coated glass surfaces at different spraying times, distances from gun nozzle to sample, and at two polymer concentration in toluene $(1 \%$ and $8 \%$ ). Air pressure was maintained at 0.7 to 0.8 bar for this batch of experiments. Higher pressure was found to result in coatings with too much solvent on target samples even for very short spraying times, and poor control over coating thickness. Even at the optimal pressure chosen for the more detailed studies, the air gun was difficult to use without quickly spraying a considerable amount of solvent and polymer on the target surfaces. PEA in toluene solutions were always prepared diluting a commercial solution that is sold by Sigma Aldrich at a concentration of around $19,6 \%$.

Experimental conditions tested are shown in table 3. Results for thickness, as measured with AFM and a qualitative description of surface morphology seen on optical microscopy, are summarised in table 4.

\begin{tabular}{|l|l|l|l|l|l|l|}
\hline $8 \%$ PEA & $400 \mathrm{~mm}$ & $500 \mathrm{~mm}$ & $700 \mathrm{~mm}$ & $1 \%$ PEA & $500 \mathrm{~mm}$ & $700 \mathrm{~mm}$ \\
\hline $5 \mathrm{~s}$ & & $\checkmark$ & & $5 \mathrm{~s}$ & & \\
\hline $8 \mathrm{~s}$ & $\checkmark$ & $\checkmark$ & $\checkmark$ & $8 \mathrm{~s}$ & $\checkmark$ & $\checkmark$ \\
\hline $30 \mathrm{~s}$ & & $\checkmark$ & $\checkmark$ & $30 \mathrm{~s}$ & $\checkmark$ & $\checkmark$ \\
\hline
\end{tabular}

Table 3. Spray conditions tested in air gun installation. Polymer in solvent concentrations at $8 \%$ or $1 \%$. Distances in $\mathrm{mm}$ are from air gun nozzle to target surfaces. Time in seconds is duration of spraying time. Air pressure at the compressed air feed was maintained at 0.7 to 0.8 bar for all experiments.

\begin{tabular}{|c|l|l|}
\hline 1\% PEA & \multicolumn{1}{|c|}{$500 \mathrm{~mm}$} & \multicolumn{1}{c|}{$700 \mathrm{~mm}$} \\
\hline $5 \mathrm{~s}$ & No measurement & No measurements \\
\hline $8 \mathrm{~s}$ & $\begin{array}{l}\text { Near full coating with textured } \\
\text { areas, ridges. Outer edge of } \\
\text { droplets 100 to 300 nm high and } \\
\text { textured droplet areas 40 to 100 } \\
\text { nm with holes. }\end{array}$ & $\begin{array}{l}\text { Partial coating } \\
\text { Heterogeneous surface with droplets } \\
\text { with ridges (outer edge of droplets } \\
\text { 200 to 300 nm high and textured } \\
\text { droplet areas 40 to 60 nm high) }\end{array}$ \\
\hline
\end{tabular}




\begin{tabular}{|c|c|c|c|c|}
\hline $30 \mathrm{~s}$ & \multicolumn{2}{|c|}{$\begin{array}{l}\text { Full coating, flat surface. } \\
\text { Thickness from } 980 \mathrm{~nm} \text { to } 1.1 \\
\mu \mathrm{m} \text {. }\end{array}$} & \multicolumn{2}{|c|}{$\begin{array}{l}\text { Partial coating } 20-30 \% \text {, } \\
\text { heterogeneous surface, with ridges } \\
\text { (outer edge of droplets } 200 \text { to } 400 \\
\text { nm high and textured droplet areas } \\
80 \text { to } 120 \mathrm{~nm} \text { high) }\end{array}$} \\
\hline 8\% PEA & $400 \mathrm{~mm}$ & \multicolumn{2}{|c|}{$500 \mathrm{~mm}$} & $700 \mathrm{~mm}$ \\
\hline $5 s$ & & \multicolumn{2}{|c|}{$\begin{array}{l}\text { Partial coating } 20-30 \% \text {, } \\
\text { with disperse droplets. } \\
\text { Thickness } 150-500 \mathrm{~nm} .\end{array}$} & \\
\hline $8 \mathrm{~s}$ & $\begin{array}{l}\text { Near full coating } \\
70-90 \% \text {. } \\
\text { Thickness from } \\
1.1 \mu \mathrm{m} \text { to } 2.1 \mu \mathrm{m} \text {. }\end{array}$ & \multicolumn{2}{|c|}{$\begin{array}{l}\text { Partial coating } 60-70 \% \text {. } \\
\text { Thickness } \begin{array}{l}\text { varies } \\
\text { greatly, } 350 \mathrm{~nm} \text { to } \\
1.5 \mu \mathrm{m} .\end{array}\end{array}$} & $\begin{array}{l}\text { Partial coating } 30-40 \% \text {, } \\
\text { heterogeneous surface, } \\
\text { disperse droplets of } \\
\text { many shapes and sizes. } \\
\text { Thickness } 320-450 \mathrm{~nm}\end{array}$ \\
\hline $30 \mathrm{~s}$ & & \multicolumn{2}{|c|}{$\begin{array}{l}\text { Full coating but very } \\
\text { heterogenous with } \\
\text { solvent drag marks. } \\
\text { Thickness around } 2 \mu \mathrm{m} \\
\text { with high variance. }\end{array}$} & $\begin{array}{l}\text { Partial coating } 70-80 \% \\
\text { with some solvent drag } \\
\text { marks. Thickness } 350 \\
\text { nm to under } 1 \mu \mathrm{m} \text {. }\end{array}$ \\
\hline \multicolumn{5}{|c|}{$\begin{array}{l}\text { Table 4. Description of surface morphology and summary of thickness } \\
\text { measurements. With spray conditions described in table } \mathrm{xx} \text {, a summary of results } \\
\text { for thickness as measured with AFM and a qualitative as measured with AFM and } \\
\text { a qualitative under optical microscopy are summarised in table } 3 \text {. }\end{array}$} \\
\hline
\end{tabular}

From these experiments we concluded that we could obtain better coatings (more homogeneous, fully covering the target surface and thin around one micron) with the solution at 1\% PEA and distances from nozzle to sample of $500 \mathrm{~mm}$. Optimal duration of spraying time is around 30 seconds, but times over 10 seconds probably produce similar thinner surfaces. Surfaces coated with the solution at $8 \%$ PEA are more difficult to produce with a flat homogeneous coating. We have seen that he size of the sprayed droplets reaching the target surface and the speed with which solvent evaporates is noticeable different for the two used polymer in solvent concentrations, but we didn't perform measurements of these properties to fully confirm if they may explain and control the quality of the surfaces produced.

In Figures 44 to 47 we show examples of the variation of surface morphology and thickness of coatings with spraying parameters. To summarise, properties of the sprayed surfaces can be controlled so that surfaces can be fully or partially coated. As expected, coating efficiency was found to be dependent on spraying time, but also very critically on distance from nozzle to target surface and very dependent on 
polymer concentration on solvent. For instance, spraying experiments with the more diluted 1\% PEA solution, may result in a surface partially covered with rather small droplets of polymer. At this concentration we see individual droplets that are usually 5 to 10 microns across with a thickness of a few hundred nanometres.

We found the size and shape of droplets to be mainly controlled with the concentration of polymer in solvent (Figures 46 and 47). Also, for both concentrations and even for shorter duration spraying experiments, droplets on the surface quickly start to coalesce onto bigger droplets that eventually coat the whole surface given sufficient spraying time. This effect seems to start at short spraying times, and, as the target surface is held vertical and perpendicular to the air gun nozzle, the bigger droplets spill over the surface before the solvent can evaporate and produce big variations in thickness and generally a heterogeneous surface for many of the conditions tested.

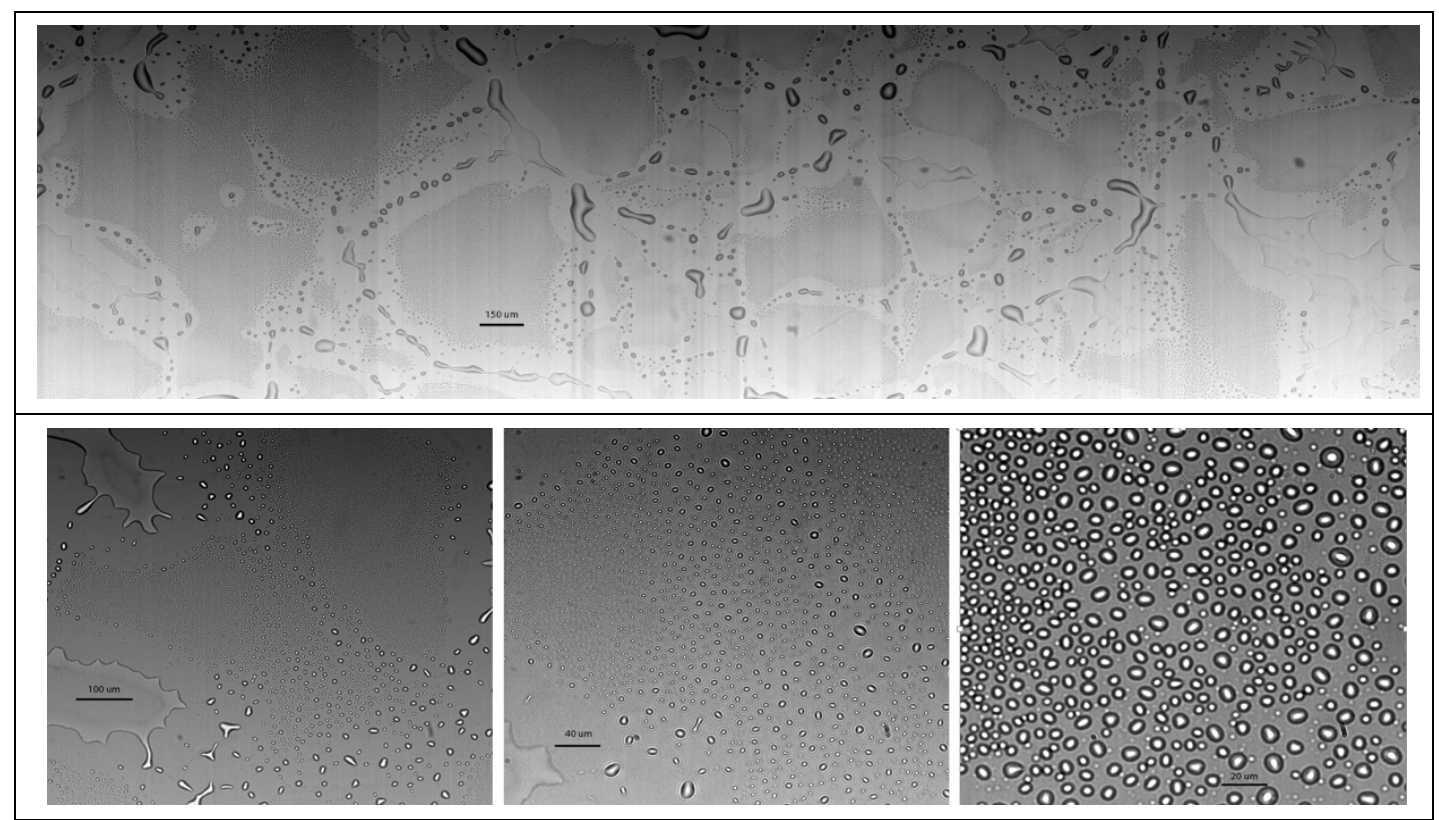

Figure 44. Airgun coatings. Optical microscopy pictures of sprayed coatings on glass surface. PEA in toluene solution (Sigma Aldrich) diluted at 1\%. Distance from nozzle to target of $500 \mathrm{~mm}$. Duration of spraying in this set was $5 \mathrm{~s}$ at 1 bar. Upper row is a composite image of 5 slides, scale bar is $150 \mu \mathrm{m}$. Bottom row shows three slides of same section at different magnifications, scale bars are 100, 40 and $20 \mu \mathrm{m}$ from left to right. 

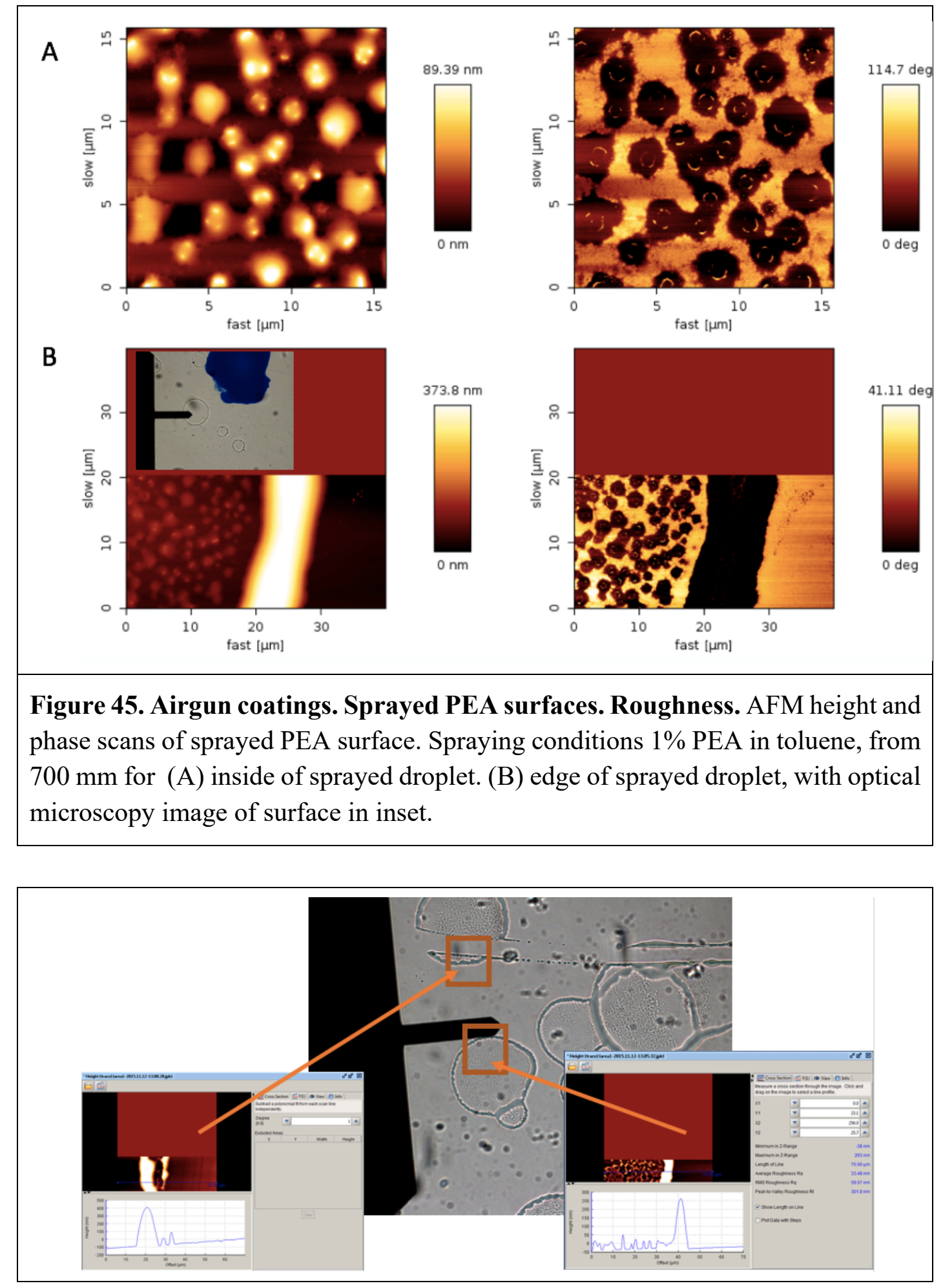


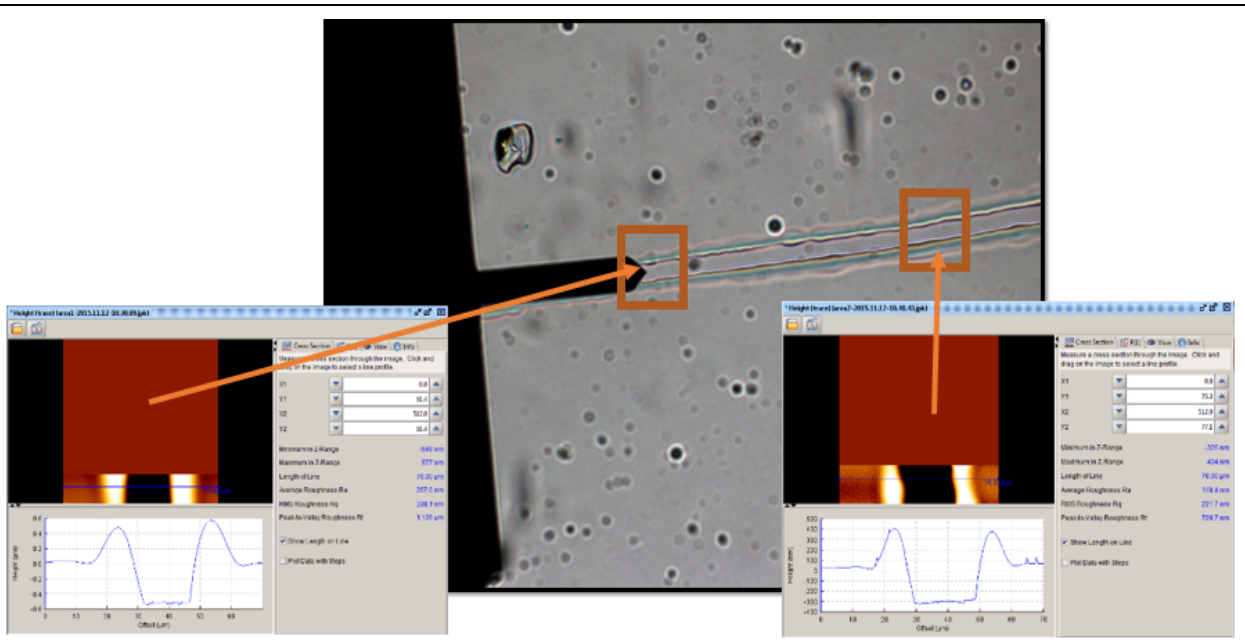

Figure 46. Airgun coatings. Sprayed PEA surfaces. Coating distribution and thickness. Scratch tests performed on samples to measure thickness of coatings. PEA at 1\%. Optical and AFM height scans (in inserts) of sprayed PEA coatings on glass coverslips. PEA is $1 \%$ in toluene sprayed at an air pressure of 0.7 to 0.8 bar. With nozzle at $700 \mathrm{~mm}$ (Top row), spraying for 8 seconds, coating is only on around $20 \%$ of the surface, in the form of splashed droplets with an, heterogeneous surface. As shown in inserts, ridges (outer edge of droplets) are about 200 to $300 \mathrm{~nm}$ high, while textured areas inside of droplets are 40 to $60 \mathrm{~nm}$ with holes. With nozzle at $500 \mathrm{~mm}$ (Bottom row), spraying for 30 seconds, coating is full and homogeneous in the form of a flat surface with a measured thickness of around $1 \mu \mathrm{m}$ in most of the surface.

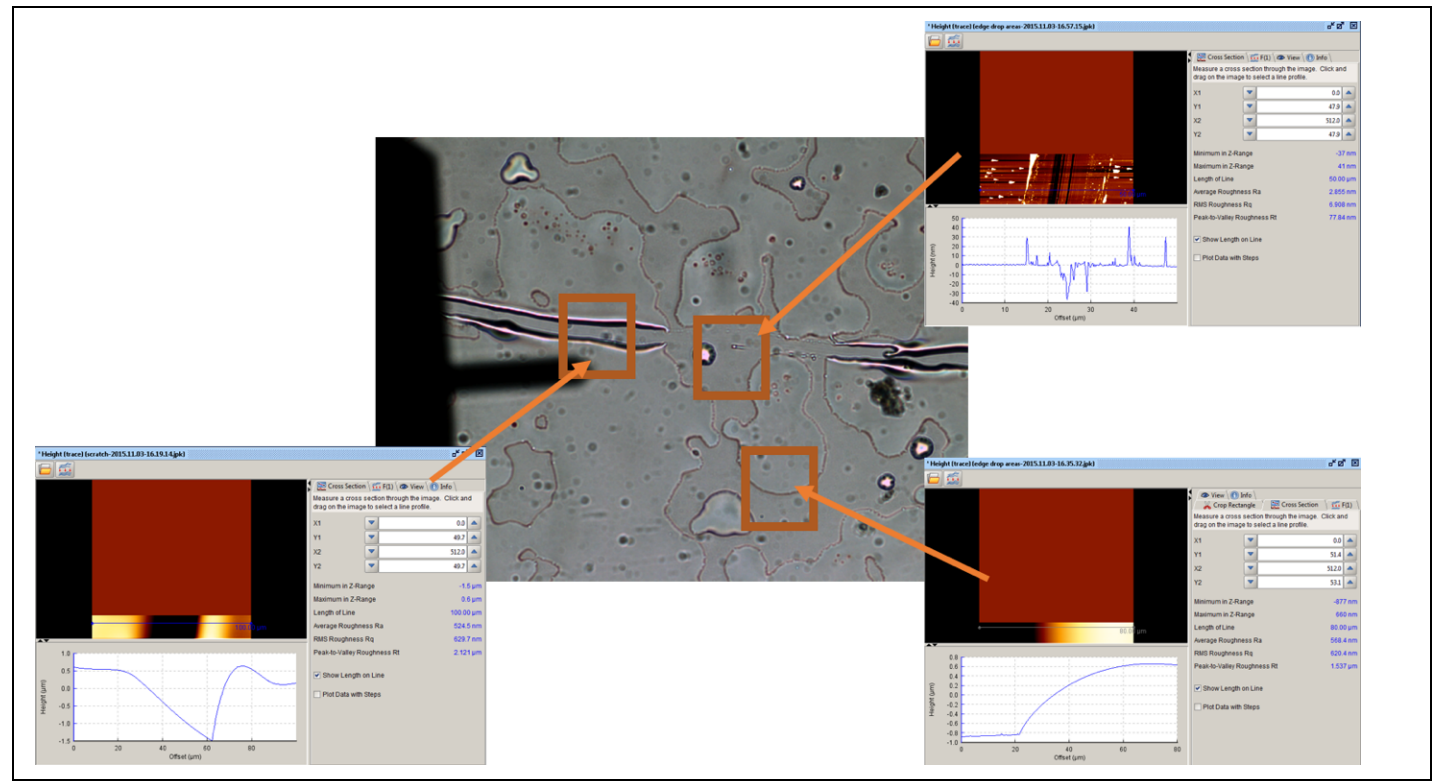




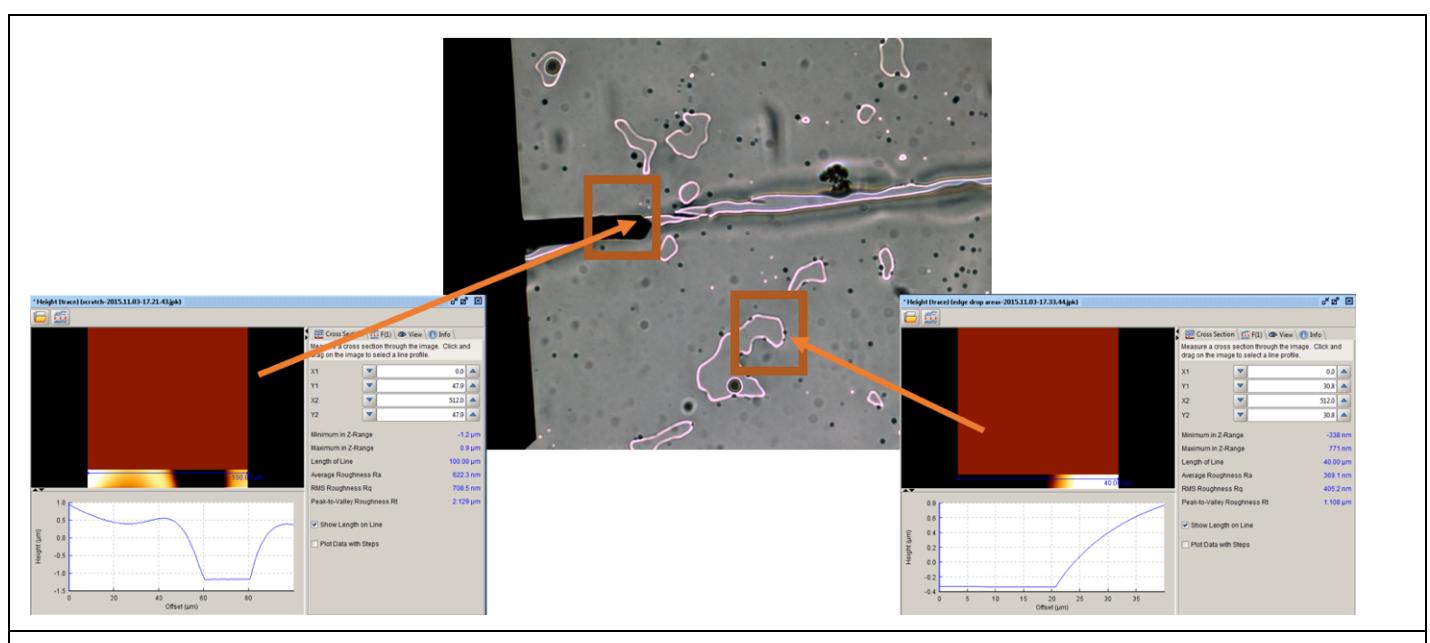

Figure 47. Airgun coatings. Sprayed PEA surfaces. Coating distribution and thickness. Scratch tests performed on samples to measure thickness of coatings. PEA at 8\%. Optical and AFM height scans (in inserts) of sprayed PEA coatings on glass coverslips. PEA is $8 \%$ in toluene sprayed at an air pressure of 0.7 to 0.8 bar. With gun nozzle at $500 \mathrm{~mm}$ from target surface and spray duration of $8 \mathrm{~s}$ results in a partial coating ( $\sim 60-70 \%)$ with a thickness that varies from $350 \mathrm{~nm}$ to $1.5 \mu \mathrm{m}$ (Top row). With nozzle at $400 \mathrm{~mm}$, spray again for 8 seconds, coating is nearly full ( $\sim 70$ $90 \%$ ) and thickness from around $1.1 \mu \mathrm{m}$ to $2.1 \mu \mathrm{m}$. Some areas of the surface seem to have two distinguishable layers of material, possibly because of droplets coalescing and solvent spilling during the spraying time (Bottom row).

\subsubsection{Airgun coatings on scaffolds}

We prepared PLLA scaffolds by a freeze extraction method and PCL scaffolds with a $3 \mathrm{D}$ printer both as described in section 2 . The goal of the experiments carried out with scaffolds in this section has been to check if our spraying installation is suitable to coat the inner surfaces of these scaffolds, and how much their structure is compromised by the toluene used to dissolve PEA.

The PLLA scaffolds are highly porous but at the same time have small pores and an irregular topology, that prevents the sprayed material to reach inner layers of the scaffold. To check how deep in the scaffold we can coat with our spraying set up we devised three simple experiments:

First, we cut a PLLA scaffold into thin slices and checked how much direct light we could see through it, with varying thickness of the slices (Figure 48). With this experiment we observe that our PLA scaffolds at a depth of $1 \mathrm{~mm}$ barely have $15 \%$ of area visible from the surface.

For a second experiment, we added sudan blue colouring to the PEA in toluene solution. and verified the coating on the scaffold samples under optical microscopy. Adjusting the focus depth of the microscope we were able to confirm the coating on the top layer of the scaffold but also see indication that the immediate inner layer of the scaffold seems to be uncoated or only partially coated (Figure 49). We also used 
this experiment to coat 3D printed PCL scaffolds (Figure 50) and confirm that the coating reaches most of their inner surfaces. The regular geometry of these scaffolds and the much larger size of the pores seems to allow a much efficient spray coating.

For our third experiment we added fluorescein to the PEA in toluene solution and verified the coating on the scaffold samples under fluorescent microscopy (Figure 48). With this experiment we confirmed that the coating reaches almost all surfaces up to around 500 to $600 \mu \mathrm{m}$ from the surface, but then almost no surface below that. We performed this experiment at a distance of $700 \mathrm{~mm}$ from nozzle to target sample, to minimise the amount of toluene reaching the scaffold surfaces, as toluene is a solvent of PLLA. We have seen that for shorter distances, at $500 \mathrm{~mm}$ for example, the scaffolds soak a visible amount of solvent and the porous structure is deformed to the point where it can collapse closing pores in the outer layers of the sprayed scaffold.

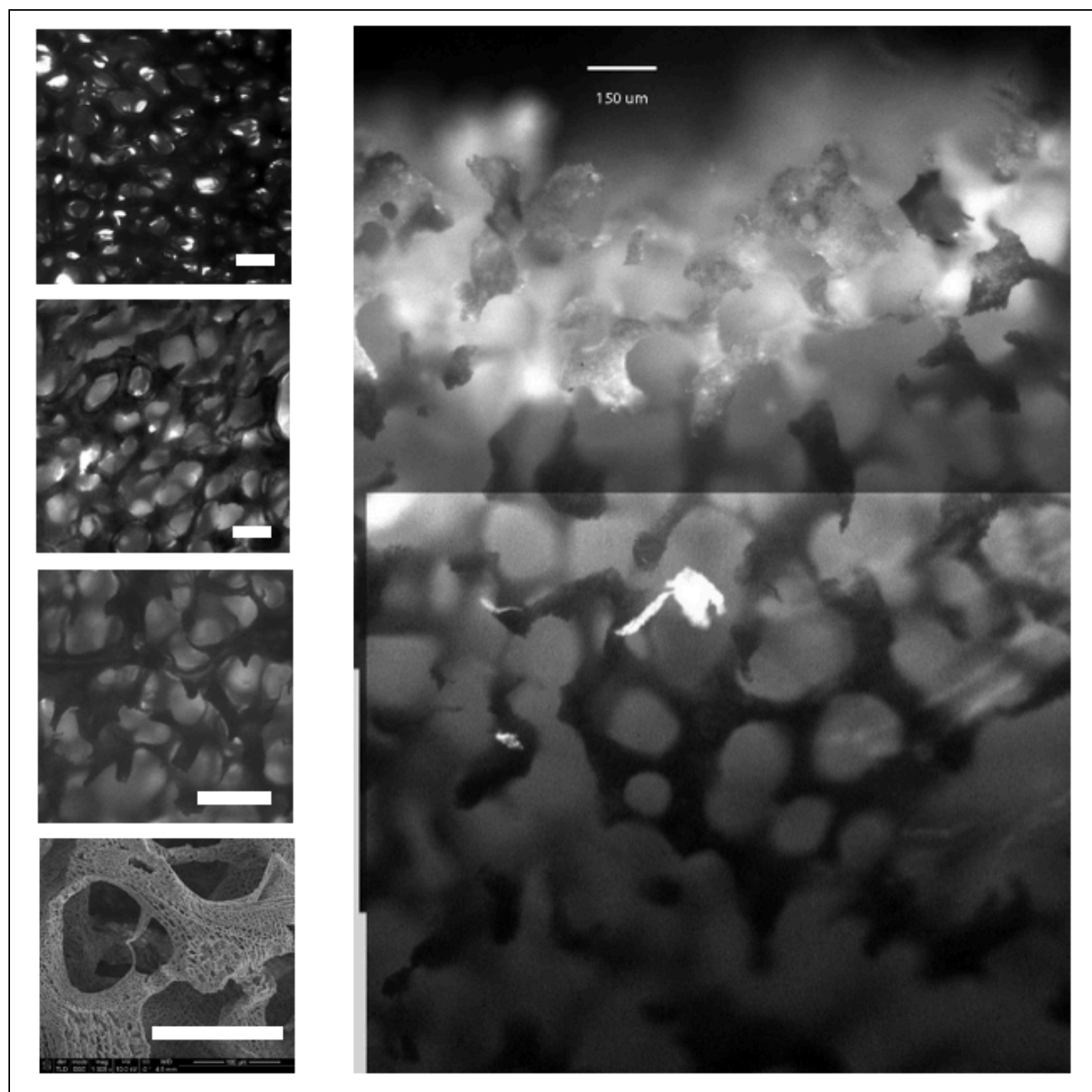

Figure 48. Airgun coatings. PLLA scaffolds coated with sprayed PEA. Left column, from top to bottom, optical microscopy pictures of slices of $1 \mathrm{~mm}, 2 \mathrm{~mm}$ and $4 \mathrm{~mm}$ of thickness, and SEM of uncoated scaffold. Even for the thinnest $1 \mathrm{~mm}$ scaffolds there's very little see-through light. Right column, composite optical microscopy picture of PLLA scaffold sprayed with PEA and fluorescein in solution. 
Nozzle at $700 \mathrm{~mm}, 1 \mathrm{bar}$, two $30 \mathrm{sec}$ sprayed layers. PEA is seen to coat the first 500-600 $\mu \mathrm{m}$ of depth from the surface of the scaffolds. All scale bars are $150 \mu \mathrm{m}$.
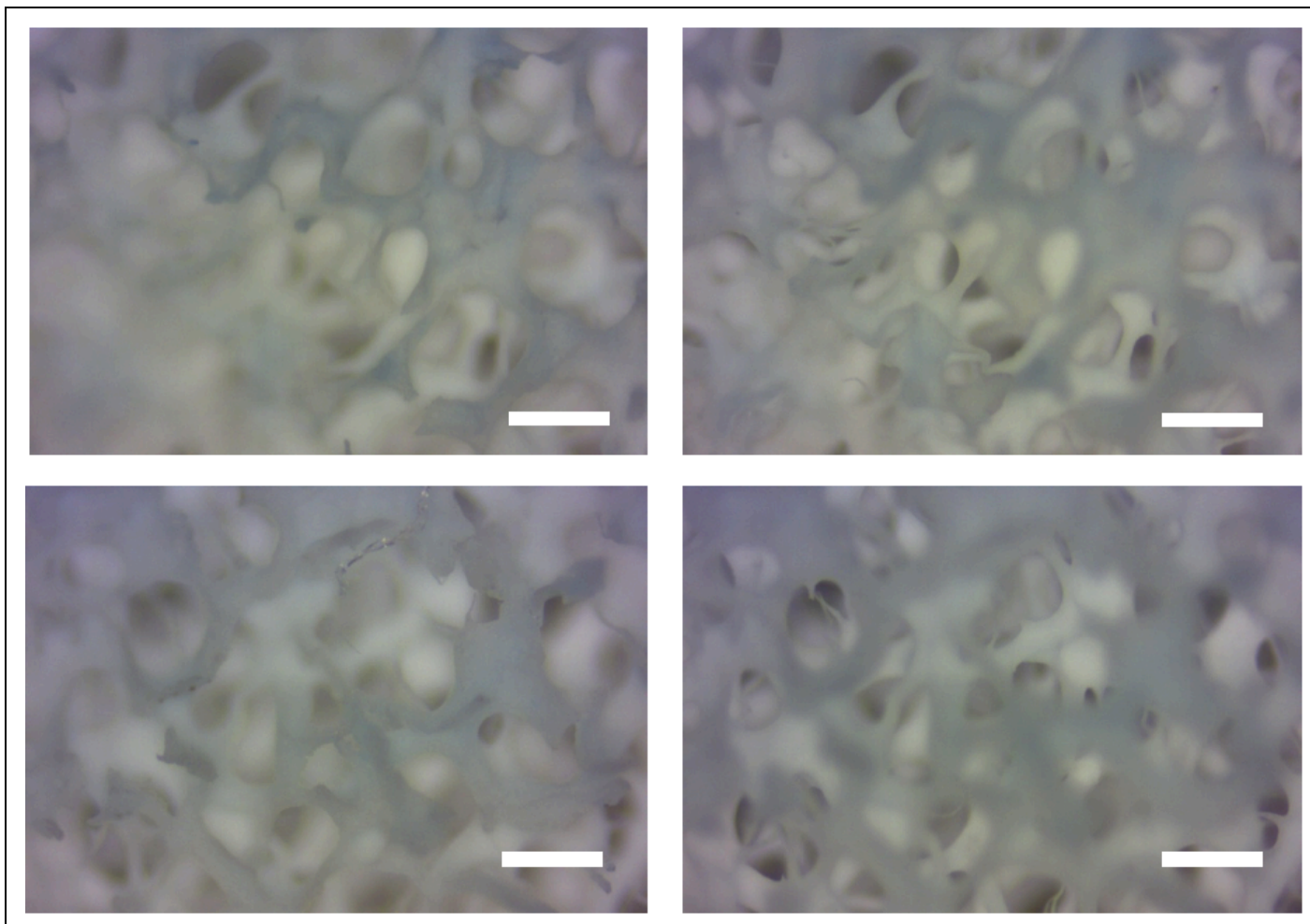

Figure 49. Airgun coatings. PLLA scaffolds coated with sprayed PEA, sudan blue. Optical microscopy picture of PLLA scaffolds manufactured with a freeze extraction technique, sprayed with nozzle at $500 \mathrm{~mm}, 1$ bar. Sudan blue in solution $(0,75 \%$ to PEA). First row pictures for scaffolds sprayed for 30 seconds with $1 \%$ PEA in solution. Image focus in top (left column) an inner (right column) surface. Second row for scaffolds sprayed for 8 seconds with $8 \%$ PEA in solution. Image focus in top an inner surface. All scale bars are $150 \mu \mathrm{m}$.

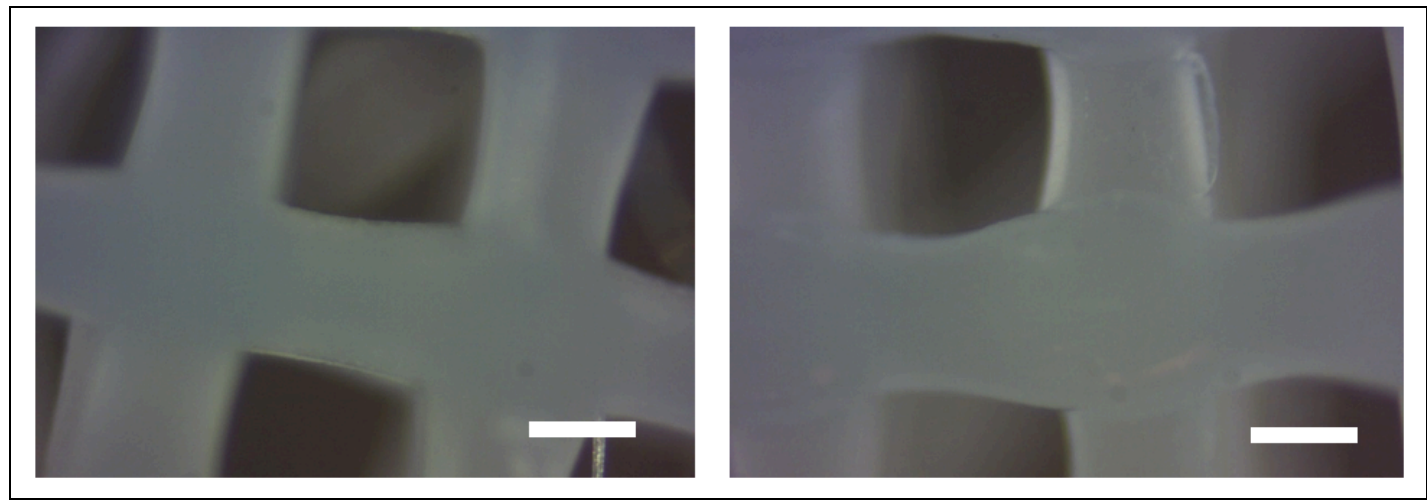

Figure 50. Airgun coatings. PCL scaffolds coated with sprayed PEA, with sudan blue colouring. Optical microscopy images of 3D printed PCL scaffold sprayed with nozzle at $500 \mathrm{~mm}, 1 \mathrm{bar}$, with sudan blue colouring in solution $(0,75 \%$ 
to PEA). (Left) Image focused on the top surface of a scaffold sprayed for 30 seconds with $1 \%$ PEA in solution. (Right) Inner surfaces are also colouring. Image focused on second row of struts of a scaffold sprayed for 8 seconds with $8 \%$ PEA in solution. All scale bars are $150 \mu \mathrm{m}$.

With these experiments we confirmed that with our simple low-cost spraying installation we are able to coat the inner surfaces of 3D printed PCL scaffolds, and how much their structure is compromised by the toluene used to dissolve PEA.

The PLLA scaffolds are highly porous but at the same time have small pores and an irregular topology, that prevents the sprayed material to reach inner layers of the scaffold. To check how deep in the scaffold we can coat with our spraying set up we devised three simple experiments:

\subsubsection{Fibronectin conformation on air gun coatings}

We adsorbed FN on sprayed surfaces to verify the protein conformation. As seen on Figures 51 and 52 below, FN adopts a compact network-like morphology on sprayed PEA surfaces. We have seen variations in the conformation of $\mathrm{FN}$ in these surfaces. Most locations we scanned seemed to show dense FN networks covering most of the surface, compared to more open FN networks seen in reference spin coated PEA surfaces (Figure 27). Sometimes in a few locations we saw more open FN networks on sprayed PEA surfaces, similar to references.

AFM scans of the surfaces with partial coatings, with disperse droplets, showed micro and nano-roughness of the surface inside of the droplets, and were sometimes difficult to produce. We didn't see a clear relation between roughness or presence of micro defects in the sprayed PEA surface and the characteristics of the FN conformation.

We saw a similar compact conformation of FN adsorbed on sprayed surfaces with different underlaying substrates, glass and PLA as shown in Figures 51 (glass) and 52 (PLA). We spin coated PLA on glass coverslips and used a thermal treatment to make it crystalline with small spherulites, with surfaces as shown in section 3.2.3.1, so that the uncoated surface was readily distinguishable with AFM. At the conditions tested sprayed PEA coating are disperse and PEA droplets cover only partly the surface. Where there was sprayed PEA the underlaying roughness of PLA was not noticeable. A denser FN conformation on sprayed PEA surfaces compared to spin coated PEA may be caused by the different topography and roughness of the surface, at a scale that affects the assembling of the FN fibrils into a network like structure. The sprayed PEA surfaces have micro and nanoscale features, in particular near the edge of the sprayed droplets as shown in Figure 45. 


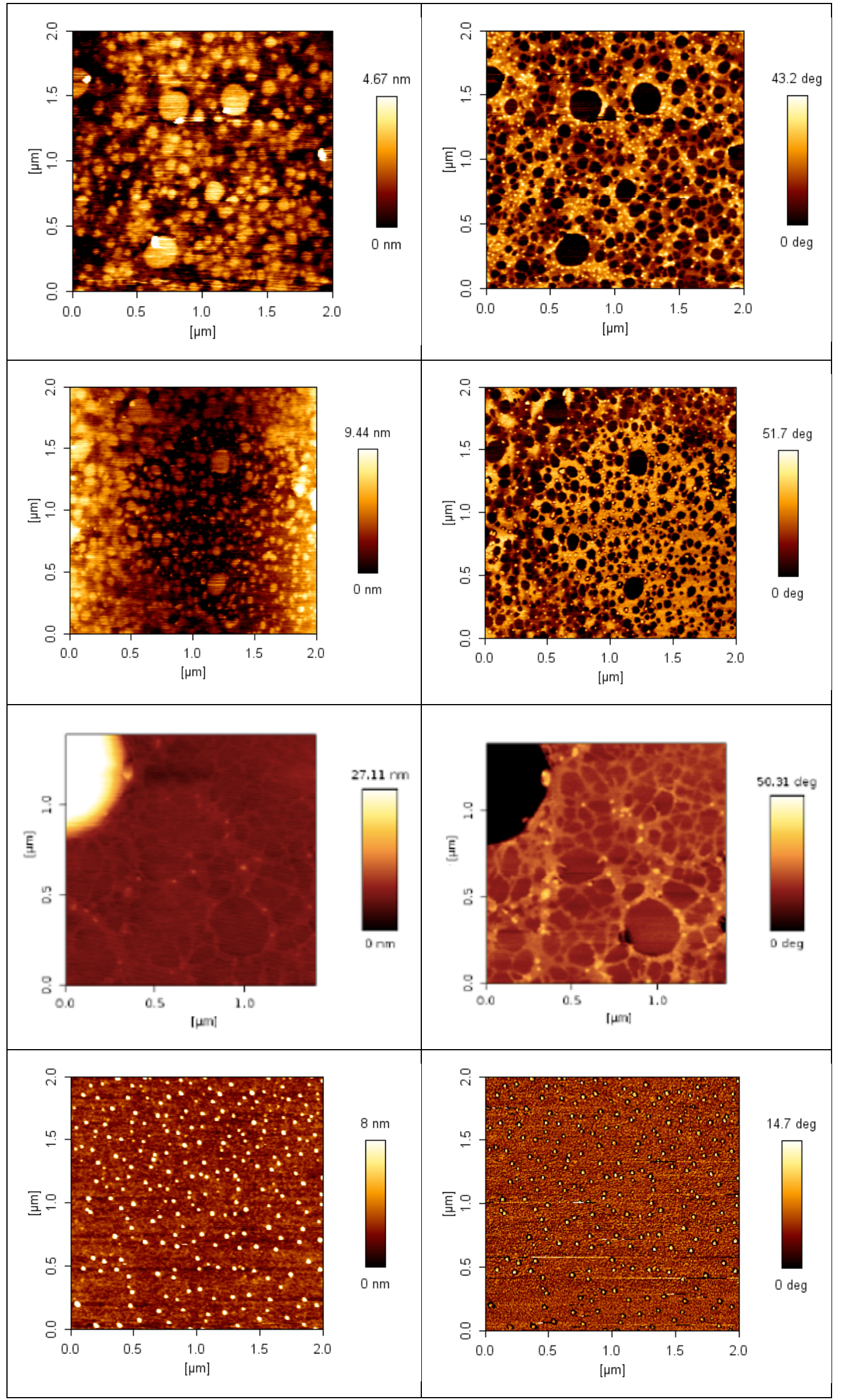


Figure 51. Airgun coatings. AFM scans of FN adsorbed on PEA sprayed on glass coverslips. AFM height (left column) and phase (right column) scans of surfaces with adsorbed FN $(20 \mu \mathrm{g} / \mathrm{ml}$ in DBPS $)$ inside a droplet of sprayed PEA (first, second and third row, three locations) and of surface outside of a sprayed droplet (bottom row). PEA is $8 \%$ in toluene, sprayed with air gun for $8 \mathrm{~s}$ at 0.7 to 0.8 bar, nozzle at $500 \mathrm{~mm}$.

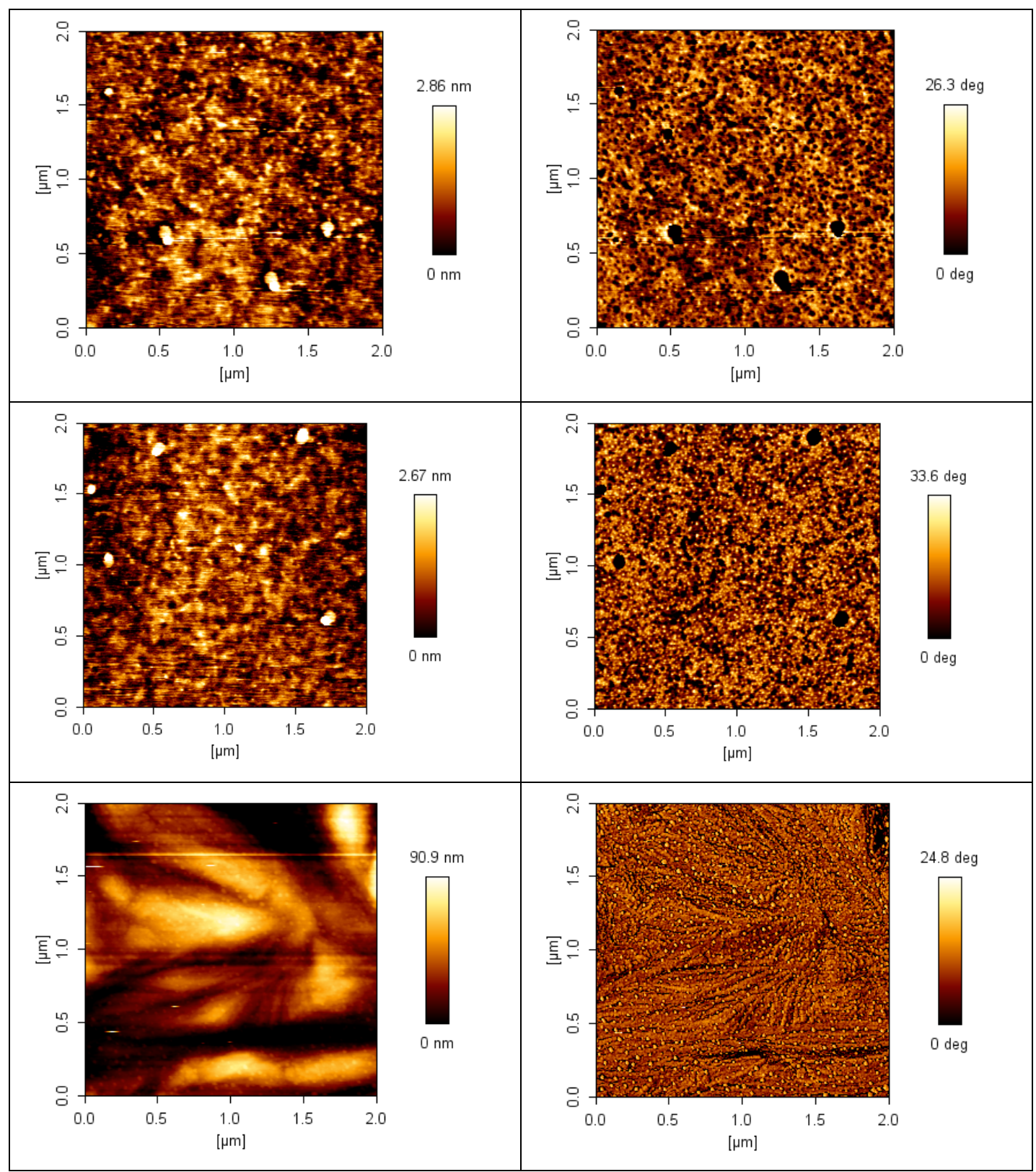




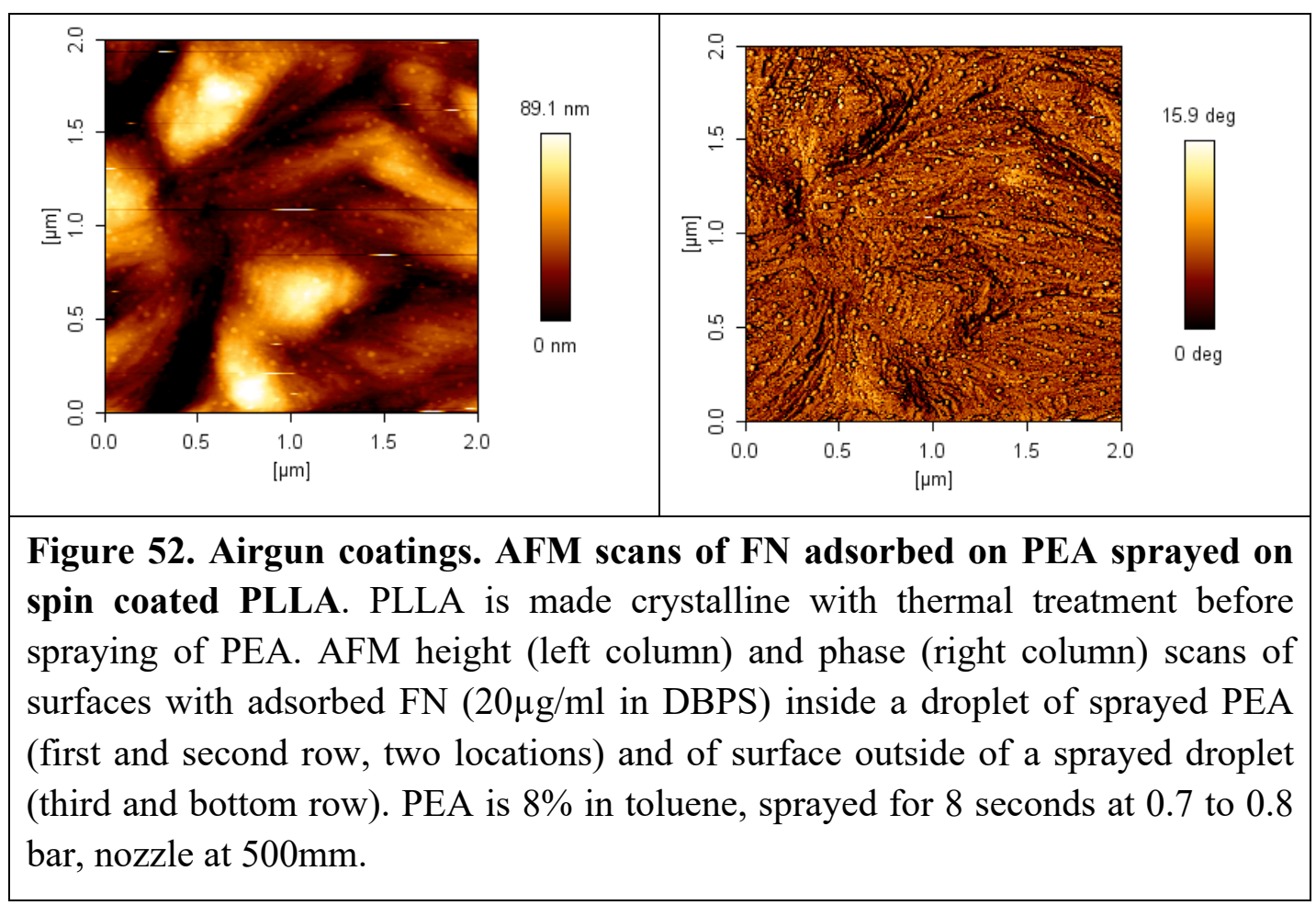

\subsection{Air brush sprayed coatings}

As with the air gun, we coated glass surfaces with our air brush installation at different spraying times, and distances from gun nozzle to sample. After a few preliminary tests we chose to spray with an $8 \%$ PEA in toluene solution, and also with a diluted solution at 3,85\% PEA solution in a mixture of ethanol and toluene. We prepared this diluted solution with a 50/50 volume mixture of ethanol and PEA in toluene at a concentration of $8 \%$. This diluted solution helped with the performance of the air brush.

With the PEA solution only in toluene we found that the air brush required exhaustive cleaning after each experiment, even after a few seconds of spraying, to avoid clogging of the air brush delicate nozzle mechanism. We found that the dilution in ethanol also helped with the evaporation of the solvent during spraying and the homogeneity of the sprayed surfaces. Air pressure was maintained at around 1 bar for this batch of experiments. Higher pressure was found to result in coatings with too much solvent on target samples and poor control over coating thickness.

Overall, the air brush was easier to use than the air gun. With this installation we found a wider practical range of pressure, distance, and spraying times were surfaces could be sprayed with repeatable and acceptable results. Only at higher pressures and shorter distances we report problems with solvent running off on the sample surfaces and heterogeneity of coatings. PEA in toluene solutions were always prepared diluting a commercial solution that is sold by Sigma Aldrich at a concentration of around 19,6\%.

Experimental conditions tested are shown with optical pictures in figures 53 and 54. Coatings sprayed with an 8\% PEA solution (figure 53) are fairly homogeneous for a 
range of tested conditions where distance from nozzle to sample is more critical than duration of experiment. At $150 \mathrm{~mm}$ of distance we find full coatings at $15 \mathrm{~s}$ or longer, and the $10 \mathrm{~s}$ experiments yield already almost full coatings. At $200 \mathrm{~mm}$ we see almost full coatings at $30 \mathrm{~s}$, but with holes and heterogeneous areas. The same kind of surface and features but enhanced are found at $300 \mathrm{~mm}$ and $30 \mathrm{~s}$. In figure 53 and 54 the conditions where a full coating is achieved are marked with a blue line.

Coatings sprayed with a PEA solution diluted in ethanol (figure 54) showed similar morphologies but with longer times to achieve a full coating. A full homogeneous coating was only produced at one tested condition, with the longest spraying time of $30 \mathrm{~s}$ and the shortest distance from nozzle to sample of $150 \mathrm{~mm}$. Much less coating reached the target surface at longer distances compared with the undiluted spraying solution. For instance, at $300 \mathrm{~mm}$ and $30 \mathrm{~s}$ there is only a very disperse number of droplets on the surfaces coated with the diluted PEA solution.

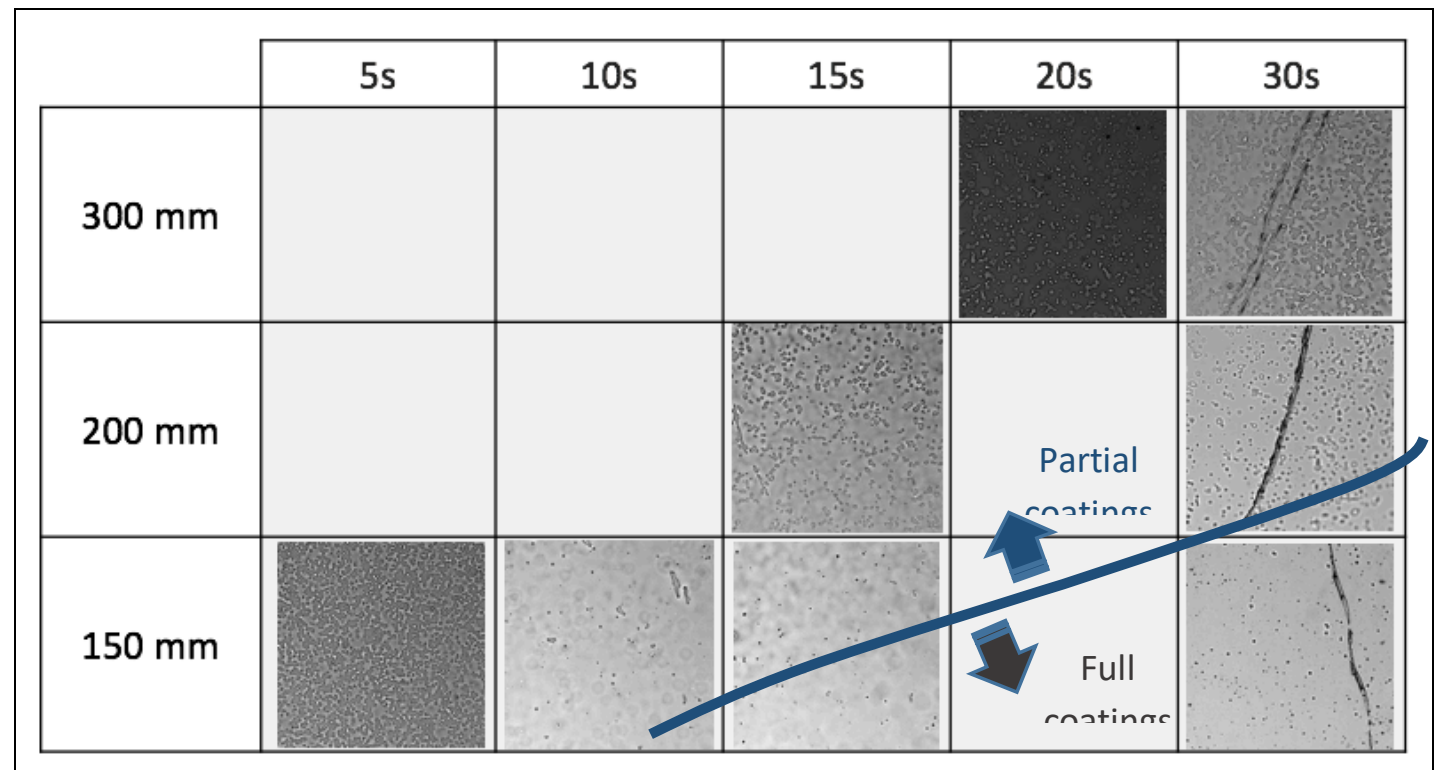

Figure 53. Airbrush coatings. Optical microscopy pictures of sprayed coatings on glass surfaces. Glass coverslips sprayed with PEA in toluene solution at a concentration of $8 \%$. Distance from nozzle of the air brush to the target surface shown in millimetres. Duration of spraying shown in seconds. All pictures at 10X magnification. 


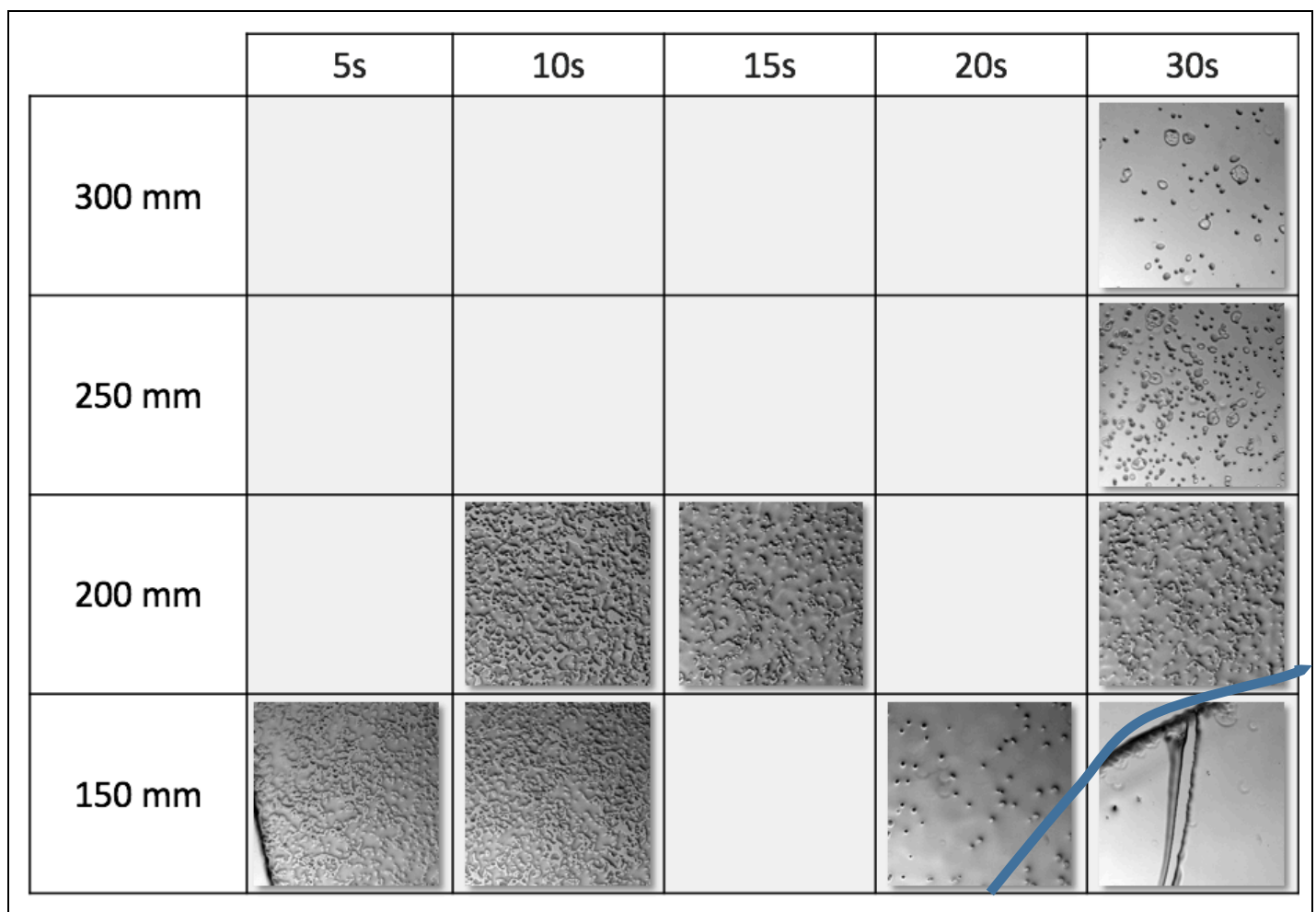

Figure 54. Airbrush coatings. Optical microscopy pictures of sprayed coatings on glass surfaces. Dilution of spraying solution in ethanol. Glass coverslips sprayed with a 50/50 volume mixture of ethanol and PEA in toluene at a concentration of $8 \%$. Distance from nozzle of the air brush to the target surface shown in millimetres. Duration of spraying shown in seconds. All pictures at $10 \mathrm{X}$ magnification.

We found easier the use of the diluted PEA solution, without clogging and sputtering problems on the air brush, and also very consistent and repeatable to achieve full coatings even if slightly longer times of spraying are required. For selected conditions with diluted PEA solution we measured thickness with an AFM scratch test and results are shown in Figure 55. 


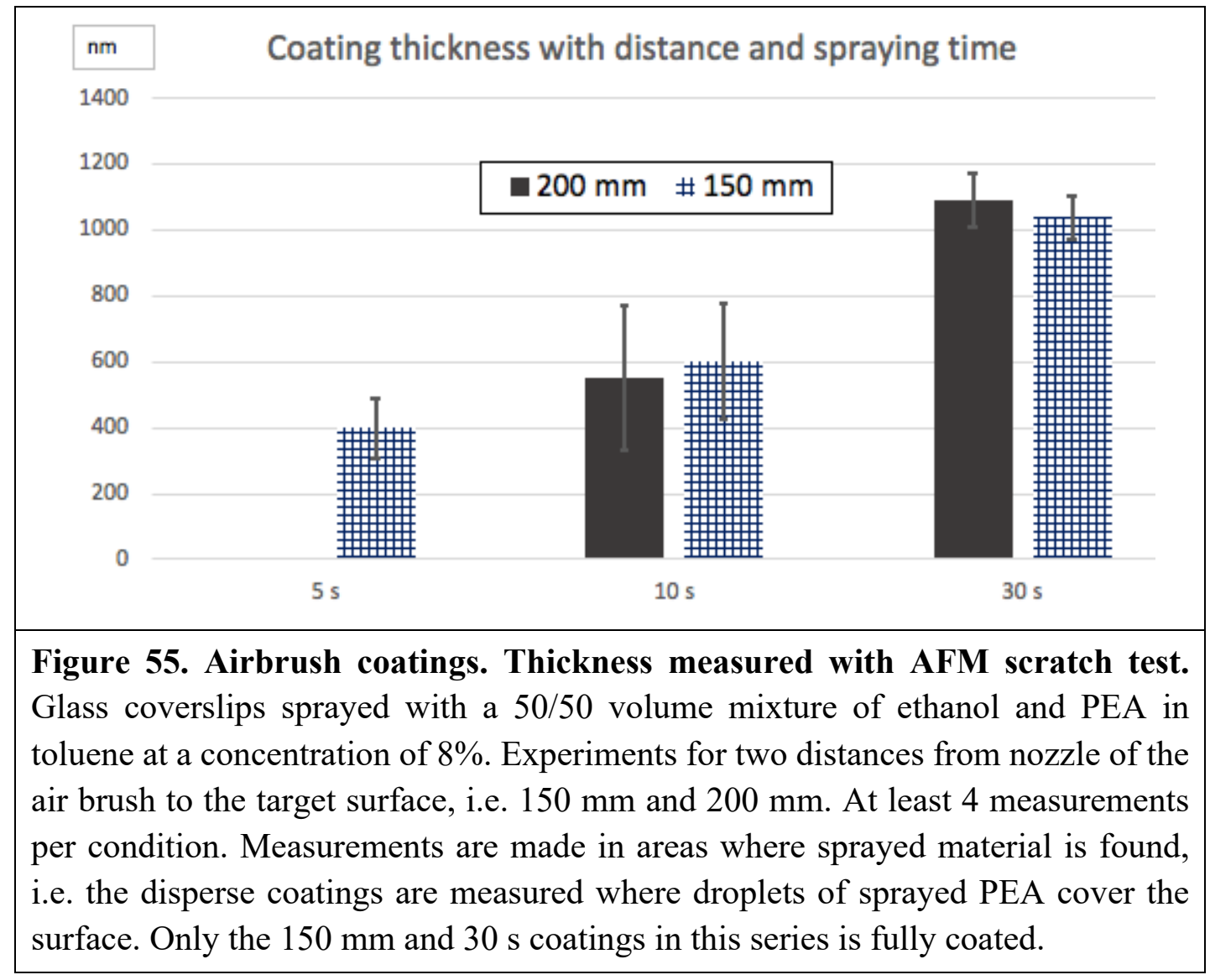

\subsubsection{Degradation of the spray coatings on flat surfaces}

We coated glass surfaces (microscopy coverslips) with our spray brush installation and characterised the stability of the surfaces after immersing in cell culture media (DPBS). We prepared samples to test four spraying conditions that yield a range of different coatings and checked the surfaces with optical microscope at several time points up to 23 days after immersion (Figure 56).

With the samples tested and images taken we are able to characterise qualitatively the degradation and its rate. Degradation is evident on all surfaces after the first week of immersion, as a loss of coating materials and exposure of underlaying surface. Degradation seems to have 'points of nucleation' in most samples but not in all of them, and holes of different sizes have appeared already at day 7 of immersion. The remaining material stays in areas of irregular shape and size. In three of the spraying conditions, A, B and C, that initially have a thicker and full coating of the surface, the degradation starts and happens quickly but by day 23 there seems to be still around $40-60 \%$ of the surface coated, with very irregular distribution of material. In the case of condition D, which initially has a partial and thinner coating, degradation seems to happen even faster and the areas that remain with coating material are smaller, and by day 23 only $10-20 \%$ of coated surface remains. 


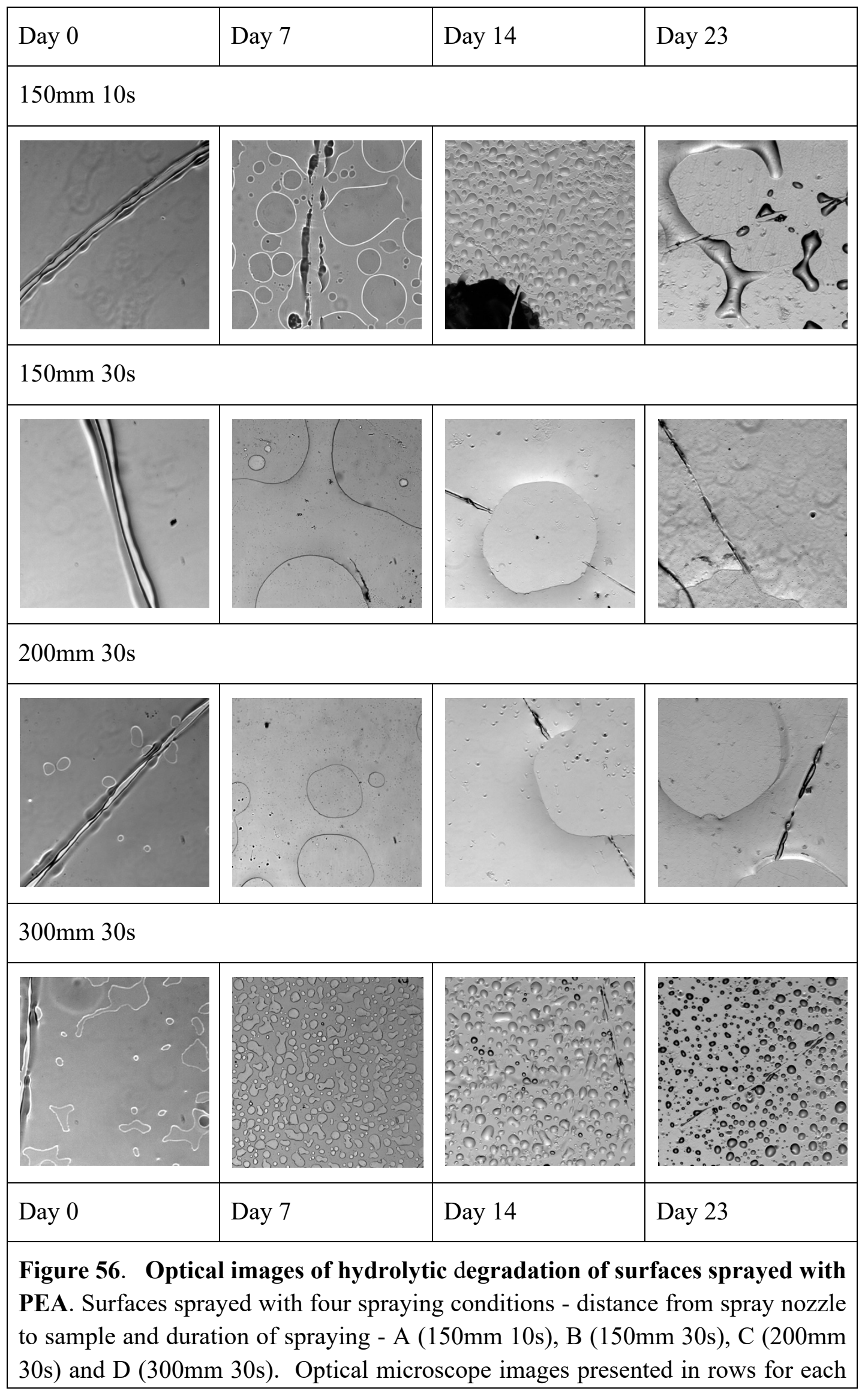


condition at four time points from preparation up to 23 days after immersion. Coatings were produced with PEA at $8 \%$ concentration in toluene. After spraying, all test samples were prepared in a sterile hood, i.e. irradiated for 15 minutes with UV light and then immersed in a 24 well plate in DPBS with added $1 \%$ of PenicillinStreptomycin antibiotic solution.

\subsubsection{Effect of coatings on the degradation of scaffolds}

This section is also relevant to the previous chapter on plasma polymerized coatings, as the experiments and results presented here relate both to spray coatings and plasma polymerized coatings on scaffolds. We measured in vitro degradation by weight loss over time in PCL scaffolds coated in several different conditions. Experiments were designed as detailed in the Materials and Methods chapter, following standard ASTM F1635.

Briefly, we measured weight loss at several time points of immersion in cell culture media of the following test groups: Uncoated (P1); plasma polymerised EA coatings, three conditions with different power and plasma time settings: Plasma 25W3m (P2), Plasma 25W15m (P3), Plasma 50W15m (P4); and PCL scaffolds with a spray coating. Spraying conditions chosen were $8 \%$ PEA in toluene, sprayed from $150 \mathrm{~mm}$ during $15 \mathrm{~s}$ each of the two sides of the scaffolds. These conditions are known to produce a full coating of around $800 \mathrm{~nm}$ to one micron in thickness. These same spray conditions have been used in an in vivo trial with a sheep model. For the degradation experiment we positioned scaffolds on a glass coverslip, to avoid touching the scaffold and minimise error when weighing samples, as the sprayed coatings are very sticky and handling of the samples can be difficult without disturbing the coatings.

Three samples of each group were periodically removed and monitored for weight loss. Initially we designed the experiment to last 6 months, with seven time points for weight loss control. We managed to run the experiment and obtain valid measurements after 7, 28 and 56 days after coating, but then most of our samples became non usable with major organic contamination. This strongly changed the $\mathrm{pH}$ of the media and probably affected the degradation rates of the samples. We couldn't tell for how long this had been going on when it was discovered around 80 days from start of the experiment, and we decided to stop the experiment and discard the remaining samples. 


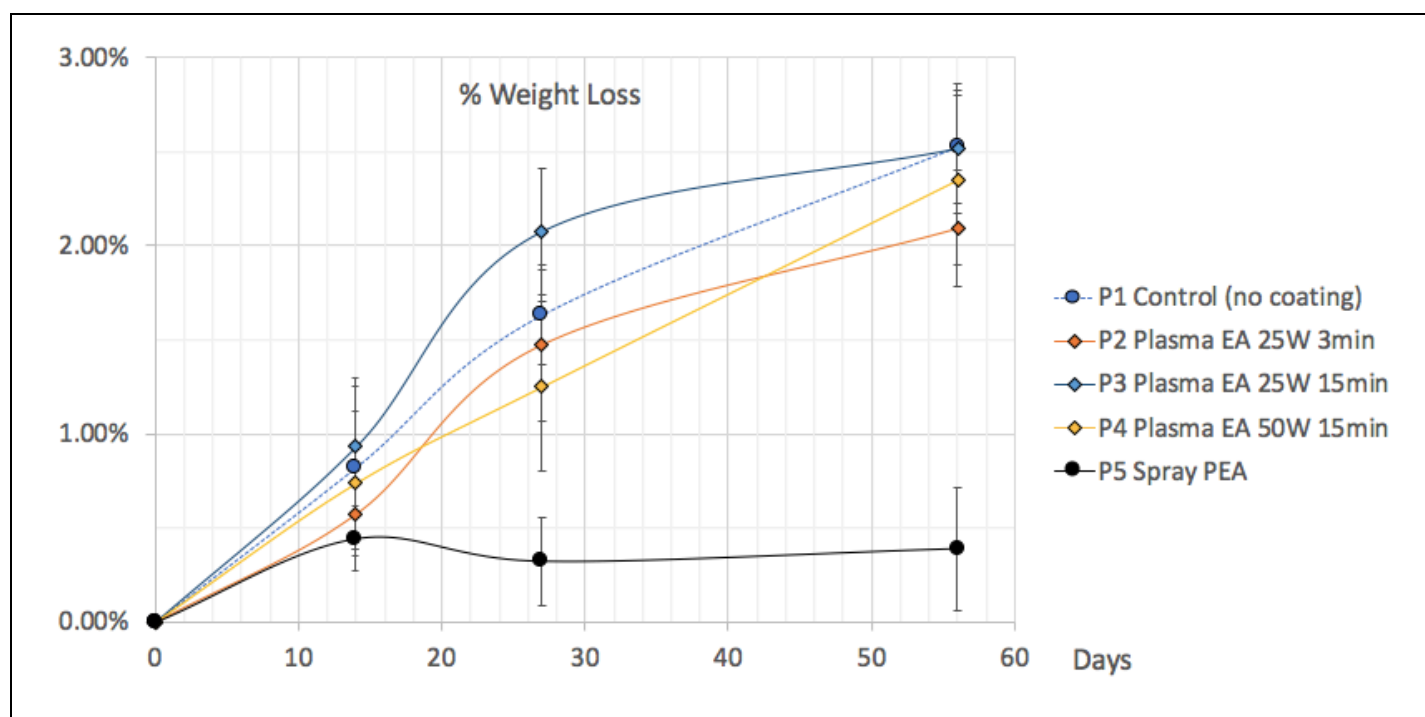

Figure 57. Degradation of coated PCL scaffolds. Scaffolds immersed in cell culture media, measured as rate of weight loss. Results are presented as an average of three measurements for each of the three samples (nine weightings) used for every time point, with brackets for standard deviation. Experimental groups are uncoated scaffolds (P1); plasma polymerised EA coated scaffolds, three conditions with different power and plasma time settings: Plasma 25W3m (P2), Plasma 25W15m (P3), Plasma 50W15m (P4); and PCL scaffolds with a spray coating (P5).

As expected, PCL degrades very slowly (Figure 57), and our scaffolds would require a longer experiment to reach complete degradation. With this test however we believe we can assess overall trends in the degradation rates (weight loss rate) and changes in material or structural properties for the tested groups (P5). Plasma deposition coatings seem to affect very little the degradation rate of the bulk material and coated scaffolds degrade at the same rate or just slightly slower than control uncoated PCL scaffolds. However, sprayed coatings have a clear differentiated effect on the scaffolds, that barely seem to degrade at all after two months. All samples in all control and experimental groups maintained full structural continuity after the experiments and we didn't see any changes in colour or shape. 


\section{Thesis Conclusion}

\subsection{Thesis conclusion in English}

In this work we have developed a plasma polymerization equipment and process that uses a low power radio frequency glow discharge to coat surfaces with plasma polymerized Poly(ethyl acrylate) (PEA). This also allows to cover porous structures such as scaffolds with a uniform coating with functional properties. Additionally, our chosen design for a plasma polymerization reactor (low pressure, low power) has demonstrated a fine control of the chemical integrity and the thickness of the coating, even at sub-micron scales. As acrylates are notably not biodegradable in bulk form or as a thick coating of more than a few hundred nanometres, their use could inhibit the functionality of biodegradable support materials such as polycaprolactone or poly(lactic-co-glycolic acid). We speculate that a very thin coating of only a few tens of nanometres, that we have shown to be feasible with our plasma reactor, can be successfully broken into pieces and cleared off by cells and their biological activity. This would allow therefore the use of the coating in applications where the substrates are biodegradable and the whole biomaterial system is required to be biodegradable.

After setting-up the equipment and testing different prototypes, we settled on the design ( $\mathrm{T}$-shaped reactor with outer ring electrodes) that best satisfied requirements for running a glow discharge at a low power range, uniformity of coating over large samples and ease of use with a high number of samples. Then our focus has been to characterise its operation and the materials produced, and how fibronectin behaves when coated onto them.

We have systematically evaluated process conditions and their impact on the plasma polymerised PEA coatings, characterising the effect of plasma power and deposition time on thickness, wettability and chemical composition of the coatings. We also have demonstrated that substrate functional roughness can be maintained after deposition of the polymer coatings. Importantly, we show that coatings deposited at different conditions all maintain a similar or better bioactivity than spin coated PEA references. We show that fibronectin assembles into compact nanonetworks on the PEA plasma polymerised coatings, with high availability of integrin and growth factor binding regions that sequester bone morphogenetic protein-2 (BMP-2). Overall, we show that this is a potent and versatile technology that can help facilitate the use of GFs in clinical applications.

In this work we built in our lab as well two custom-made installations to spray PEA from solutions in toluene on surfaces and scaffolds, as a complementary coating technology. Our first pilot installation was for a simple high-volume airgun. A second installation that allowed more precise control of the coating was for a low volume airbrush. We optimised protocols and requirements to coat surfaces and scaffolds for the two spraying installations, we developed and characterised the sprayed coatings (thickness, partial/full coating distribution, degradation in water), checked the 
conformation of fibronectin on sprayed surfaces with AFM, and verified the efficiency in coating 3D structures with pores and the effect on degradation rate of a biodegradable substrate (PCL).

\subsection{Conclusiones de la tesis en Español}

En este trabajo hemos desarrollado un equipo y proceso de polimerización por plasma que utiliza una descarga luminiscente de radiofrecuencia de baja potencia para recubrir superficies con poli(etil acrilato) (PEA) polimerizado por plasma. Esto también permite cubrir estructuras porosas como scaffolds con un recubrimiento uniforme con propiedades funcionales. Además, nuestro diseño elegido de reactor de polimerización por plasma (baja presión, baja potencia) ha demostrado un control fino de la integridad química y el grosor del recubrimiento, incluso a escalas submicrométricas. Como los acrilatos no son especialmente biodegradables como material de base o como un recubrimiento grueso de más de unos pocos cientos de nanómetros, su uso podría inhibir la funcionalidad de otros materiales de soporte biodegradables como la policaprolactona o el poli (ácido láctico-co-glicólico). Una capa muy delgada de solo unas pocas decenas de nanómetros, que hemos demostrado que es factible con nuestro reactor de plasma, especulamos que puede romperse con éxito en pedazos y eliminarse por las células y su actividad biológica. Esto permitiría el uso del recubrimiento en aplicaciones donde los sustratos son biodegradables y se requiere que todo el sistema de biomateriales sea biodegradable.

Después de configurar el equipo y probar diferentes prototipos, nos decidimos por el diseño (reactor en forma de $\mathrm{T}$ con electrodos de anillo externos) que mejor satisficiera los requisitos de ejecutar una descarga luminiscente en un rango de baja potencia, uniformidad de recubrimiento sobre muestras grandes, y facilidad de utilizar con una gran cantidad de muestras. Tras esto nuestro objetivo ha sido caracterizar el funcionamiento del equipo y de los materiales producidos, incluyendo el comportamiento de la fibronectina cuando se recubre sobre ellos.

Hemos evaluado sistemáticamente las condiciones del proceso y su impacto en los recubrimientos de PEA polimerizados por plasma, caracterizando el efecto de la potencia del plasma y el tiempo de deposición, en el espesor, la hidrofilicidad y la composición química de los recubrimientos. También hemos demostrado que la rugosidad funcional del sustrato se puede mantener después de la deposición de los recubrimientos poliméricos. Es importante destacar que mostramos que los recubrimientos depositados en diferentes condiciones mantienen una bioactividad similar o mejor que las referencias de PEA recubiertas por spin coating. Mostramos que la fibronectina, sobre los recubrimientos polimerizados con plasma de PEA, se ensambla en estructuras de aspecto de redes compactas de tamaño nanométrico, con alta disponibilidad de dominios de unión a integrinas y factores de crecimiento que secuestran la proteína morfogenética ósea 2 (BMP-2). En general, mostramos que esta es una tecnología potente y versátil que puede ayudar a facilitar el uso de GF en aplicaciones clínicas. 
En este trabajo, también hemos construido en nuestro laboratorio dos instalaciones personalizadas para pulverizar (spray) PEA a partir de soluciones en tolueno en superficies y scaffolds, como tecnología de recubrimiento complementaria. Nuestra primera instalación piloto fue una pistola de aire sencilla de alto volumen. Una segunda instalación, que permitió un control más preciso del recubrimiento, fue para un aerógrafo de bajo volumen. Optimizamos los protocolos y requisitos para recubrir superficies y scaffolds para las dos instalaciones de pulverización, desarrollamos y caracterizamos los recubrimientos pulverizados (espesor, distribución parcial / total del recubrimiento, degradación en agua), verificamos la conformación de fibronectina en superficies pulverizadas con AFM y verificamos la eficiencia en el recubrimiento de estructuras 3D con poros y el efecto sobre la tasa de degradación de un sustrato biodegradable (policaprolactona).

\subsection{Conclusions de la tesis en Valencià}

En aquest treball hem desenvolupat un equip i procés de polimerització per plasma que utilitza una descàrrega luminiscent de radiofreqüència de baixa potència per recobrir superfícies amb poli (etil acrilat) (PEA) polimeritzat per plasma. Això també permet cobrir estructures poroses com scaffolds amb un recobriment uniforme amb propietats funcionals. A més, el nostre disseny escollit de reactor de polimerització per plasma (baixa pressió, baixa potència) ha demostrat un control fi de la integritat química $\mathrm{i}$ el gruix de l'recobriment, fins i tot a escales submicromètriques. Com els acrilats no són especialment biodegradables com a material de base o com un recobriment gruix de més d'uns pocs centenars de nanòmetres, el seu ús podria inhibir la funcionalitat d'altres materials de suport biodegradables com la policaprolactona o el poli (àcid làctic-co-glicòlic ). Una capa molt delgada de només unes poques desenes de nanòmetres, que hem demostrat que és factible amb el nostre reactor de plasma, especulem que pot trencar-se amb èxit en trossos i eliminar-se per les cèl·lules i la seva activitat biològica. Això permetria l'ús del recobriment en aplicacions on els substrats són biodegradables i es requereix que tot el sistema de biomaterials sigui biodegradable.

Després de configurar l'equip i provar diferents prototips, ens vam decidir pel disseny (reactor en forma de $\mathrm{T}$ amb elèctrodes d'anell externs) que millor satisfés els requisits d'executar una descàrrega luminescent en un rang de baixa potència, uniformitat de recobriment sobre mostres grans, i facilitat d'utilitzar amb una gran quantitat de mostres. Després d'això el nostre objectiu ha estat caracteritzar el funcionament de l'equip i dels materials produits, incloent el comportament de la fibronectina quan es recobreix sobre ells.

Hem avaluat sistemàticament les condicions del procés i el seu impacte en els recobriments de PEA polimeritzats per plasma, caracteritzant l'efecte de la potència del plasma i el temps de deposició, al gruix, la hidrofilicitat i la composició química dels recobriments. També hem demostrat que la rugositat funcional del substrat es pot mantenir després de la deposició dels recobriments polimèrics. És important destacar 
que vam mostrar que els recobriments dipositats en diferents condicions mantenen una bioactivitat similar o millor que les referències de PEA recobertes per spin coating. Mostrem que la fibronectina, sobre els recobriments polimeritzats amb plasma d'PEA, s'acobla en estructures d'aspecte de xarxes compactes de mida nanomètrica, amb alta disponibilitat de dominis d'unió a integrines $\mathrm{i}$ factors de creixement que segresten la proteïna morfogenètica òssia 2 (BMP- 2). En general, vam mostrar que aquesta és una tecnologia potent $\mathrm{i}$ versàtil que pot ajudar a facilitar l'ús de GF en aplicacions clíniques.

En aquest treball, també hem construït el nostre laboratori dues instal-lacions personalitzades per polvoritzar (esprai) PEA a partir de solucions en toluè en superfícies i scaffolds, com a tecnologia de recobriment complementària. La nostra primera instal·lació pilot va ser una pistola d'aire senzilla d'alt volum. Una segona instal·lació, que va permetre un control més precís del recobriment, va ser per a un aerògraf de baix volum. Optimitzem els protocols i requisits per recobrir superfícies i scaffolds per a les dues instal·lacions de polvorització, desenvolupem i caracteritzem els recobriments polvoritzats (gruix, distribució parcial / total del recobriment, degradació en aigua), verifiquem la conformació de fibronectina en superfícies polvoritzades amb AFM i verifiquem l'eficiència en el recobriment d'estructures 3D amb porus i l'efecte sobre la taxa de degradació d'un substrat biodegradable (policaprolactona). 


\section{References}

[1] N. K. Karamanos, "Special Issue: Extracellular matrix in health and disease," FEBS J., vol. 286, no. 15, pp. 2823-3075, Aug. 2019.

[2] G. S. Hussey, J. L. Dziki, and S. F. Badylak, "Extracellular matrix-based materials for regenerative medicine," Nat. Rev. Mater., vol. 3, no. 7, pp. 159173, 2018.

[3] K. S. Hellmund and B. Koksch, "Self-Assembling Peptides as Extracellular Matrix Mimics to Influence Stem Cell's Fate ," Frontiers in Chemistry, vol. 7. p. $172,2019$.

[4] A. J. Zollinger and M. L. Smith, "Fibronectin, the extracellular glue," Matrix Biol., vol. 60-61, pp. 27-37, 2017.

[5] G. Baneyx and V. Vogel, "Self-assembly of fibronectin into fibrillar networks underneath dipalmitoyl phosphatidylcholine monolayers: role of lipid matrix and tensile forces," Proc. Natl. Acad. Sci. U. S. A., vol. 96, no. 22, pp. 12518 12523 , Oct. 1999.

[6] V. Llopis-Hernández et al., "Material-driven fibronectin assembly for highefficiency presentation of growth factors," Sci. $A d v$., vol. 2, no. 8, p. e1600188, Aug. 2016.

[7] Z. A. Cheng et al., "Nanoscale Coatings for Ultralow Dose BMP-2-Driven Regeneration of Critical-Sized Bone Defects," $A d v$. Sci., vol. 6, no. 2, p. 1800361, Jan. 2019.

[8] M. Salmerón-Sánchez and M. J. Dalby, "Synergistic growth factor microenvironments," Chem. Commun., vol. 52, no. 91, pp. 13327-13336, 2016.

[9] M. Ehrlich, "Endocytosis and trafficking of BMP receptors: Regulatory mechanisms for fine-tuning the signaling response in different cellular contexts," Cytokine Growth Factor Rev., vol. 27, pp. 35-42, 2016.

[10] K. S. Cahill, J. H. Chi, A. Day, and E. B. Claus, "Prevalence, Complications, and Hospital Charges Associated With Use of Bone-Morphogenetic Proteins in Spinal Fusion Procedures," JAMA, vol. 302, no. 1, pp. 58-66, Jul. 2009.

[11] A. Faundez, C. Tournier, M. Garcia, S. Aunoble, and J.-C. Le Huec, "Bone morphogenetic protein use in spine surgery-complications and outcomes: a systematic review," Int. Orthop., vol. 40, no. 6, pp. 1309-1319, 2016.

[12] M. J. Dalby, A. J. García, and M. Salmeron-Sanchez, "Receptor control in mesenchymal stem cell engineering," Nat. Rev. Mater., vol. 3, no. 3, p. 17091, 2018

[13] M. M. Martino and J. A. Hubbell, "The 12th-14th type III repeats of fibronectin function as a highly promiscuous growth factor-binding domain," FASEB J., vol. 24, no. 12, pp. 4711-4721, Jul. 2010.

[14] L. Chen, C. Yan, and Z. Zheng, "Functional polymer surfaces for controlling cell behaviors," Mater. Today, vol. 21, no. 1, pp. 38-59, 2018.

[15] R. A. Surmenev, M. A. Surmeneva, and A. A. Ivanova, "Significance of calcium phosphate coatings for the enhancement of new bone osteogenesis - A review," Acta Biomater., vol. 10, no. 2, pp. 557-579, 2014.

[16] R. Agarwal and A. J. García, "Biomaterial strategies for engineering implants for enhanced osseointegration and bone repair.," Adv. Drug Deliv. Rev., vol. 94, pp. 53-62, Nov. 2015. 
[17] M. J. Dalby, N. Gadegaard, and R. O. C. Oreffo, "Harnessing nanotopography and integrin-matrix interactions to influence stem cell fate," Nat. Mater., vol. 13, no. 6, pp. 558-569, Jun. 2014.

[18] Z. Chen et al., "Tuning Chemistry and Topography of Nanoengineered Surfaces to Manipulate Immune Response for Bone Regeneration Applications," ACS Nano, vol. 11, no. 5, pp. 4494-4506, May 2017.

[19] M. Hoyos-Nogués, E. Falgueras-Batlle, M.-P. Ginebra, J. Manero, J. Gil, and C. Mas-Moruno, "A Dual Molecular Biointerface Combining RGD and KRSR Sequences Improves Osteoblastic Functions by Synergizing Integrin and CellMembrane Proteoglycan Binding," Int. J. Mol. Sci., vol. 20, no. 6, p. 1429, Mar. 2019.

[20] N. R. Gandavarapu, P. D. Mariner, M. P. Schwartz, and K. S. Anseth, "Extracellular matrix protein adsorption to phosphate-functionalized gels from serum promotes osteogenic differentiation of human mesenchymal stem cells," Acta Biomater., vol. 9, no. 1, pp. 4525-4534, 2013.

[21] G. Gigliobianco, C. K. Chong, and S. MacNeil, "Simple surface coating of electrospun poly-L-lactic acid scaffolds to induce angiogenesis," J. Biomater. Appl., vol. 30, no. 1, pp. 50-60, Feb. 2015.

[22] M. Kim, K. S. Schmitt, W. J. Choi, D. J. Krutty, and P. Gopalan, "From SelfAssembled Monolayers to Coatings: Advances in the Synthesis and Nanobio Applications of Polymer Brushes," Polymers , vol. 7, no. 7. 2015.

[23] L. Hao et al., "Directing the fate of human and mouse mesenchymal stem cells by hydroxyl-methyl mixed self-assembled monolayers with varying wettability," J. Mater. Chem. B, vol. 2, no. 30, p. 4794, Jun. 2014.

[24] M. R. Sprott, G. Gallego-Ferrer, M. J. Dalby, M. Salmerón-Sánchez, and M. Cantini, "Functionalization of PLLA with Polymer Brushes to Trigger the Assembly of Fibronectin into Nanonetworks," Adv. Healthc. Mater., p. 1801469, Jan. 2019.

[25] E. J. Tobin, "Recent coating developments for combination devices in orthopedic and dental applications: A literature review," Adv. Drug Deliv. Rev., vol. 112, pp. 88-100, 2017.

[26] N. J. Hickok and I. M. Shapiro, "Immobilized antibiotics to prevent orthopaedic implant infections," Adv. Drug Deliv. Rev., vol. 64, no. 12, pp. 1165-1176, 2012.

[27] M. M. Martino, P. S. Briquez, K. Maruyama, and J. A. Hubbell, "Extracellular matrix-inspired growth factor delivery systems for bone regeneration," $A d v$. Drug Deliv. Rev., vol. 94, pp. 41-52, 2015.

[28] M. R. Newman and D. S. Benoit, "Local and targeted drug delivery for bone regeneration," Curr. Opin. Biotechnol., vol. 40, pp. 125-132, 2016.

[29] N. J. Shah et al., "Surface-mediated bone tissue morphogenesis from tunable nanolayered implant coatings.," Sci. Transl. Med., vol. 5, no. 191, p. 191ra83, Jun. 2013.

[30] M. L. Macdonald, R. E. Samuel, N. J. Shah, R. F. Padera, Y. M. Beben, and P. T. Hammond, "Tissue integration of growth factor-eluting layer-by-layer polyelectrolyte multilayer coated implants," Biomaterials, vol. 32, no. 5, pp. 1446-1453, 2011.

[31] J. Almodóvar et al., "Spatial patterning of BMP-2 and BMP-7 on biopolymeric 
films and the guidance of muscle cell fate," Biomaterials, vol. 35, no. 13, pp. 3975-3985, 2014.

[32] S. G. Caridade et al., "Myoconductive and osteoinductive free-standing polysaccharide membranes," Acta Biomater., vol. 15, pp. 139-149, 2015.

[33] H. Yasuda and T. S. Hsu, "Some aspects of plasma polymerization of fluorinecontaining organic compounds," J. Polym. Sci. Polym. Chem. Ed., vol. 15, no. 10, pp. 2411-2425, Oct. 1977.

[34] H. Yasuda and M. Gazicki, "Biomedical applications of plasma polymerization and plasma treatment of polymer surfaces," Biomaterials, vol. 3, no. 2, pp. 6877, Apr. 1982.

[35] F. F. Shi, "Recent advances in polymer thin films prepared by plasma polymerization Synthesis, structural characterization, properties and applications," Surf. Coatings Technol., vol. 82, no. 1-2, pp. 1-15, Jul. 1996.

[36] K. Vasilev et al., "Early Stages of Growth of Plasma Polymer Coatings Deposited from Nitrogen- and Oxygen-Containing Monomers," Plasma Process. Polym., vol. 7, no. 9-10, pp. 824-835, Oct. 2010.

[37] C. Gabler et al., "Evaluation of osseointegration of titanium alloyed implants modified by plasma polymerization.," Int. J. Mol. Sci., vol. 15, no. 2, pp. 245464, Jan. 2014.

[38] X. Liu, Q. Feng, A. Bachhuka, and K. Vasilev, "Surface modification by allylamine plasma polymerization promotes osteogenic differentiation of human adipose-derived stem cells.," ACS Appl. Mater. Interfaces, vol. 6, no. 12, pp. 9733-41, Jun. 2014.

[39] Y. Zheng, C. Xiong, and L. Zhang, "Dose-dependent enhancement of osteoblast cell adhesion, spreading and proliferation on plasma-carboxylated poly(etheretherketone) surface," Mater. Lett., vol. 164, pp. 60-63, 2016.

[40] P. Cools, N. De Geyter, E. Vanderleyden, F. Barberis, P. Dubruel, and R. Morent, "Adhesion improvement at the PMMA bone cement-titanium implant interface using methyl methacrylate atmospheric pressure plasma polymerization," Surf. Coatings Technol., vol. 294, pp. 201-209, 2016.

[41] R. Bitar, P. Cools, N. De Geyter, and R. Morent, "Acrylic acid plasma polymerization for biomedical use," Appl. Surf. Sci., vol. 448, pp. 168-185, 2018.

[42] "Ethyl Acrylate technical data sheet - Form No. 745-00108-1004-AA." Dow Chemical.

[43] CRC Handbook of Chemistry and Physics.

[44] "Database., National Center for Biotechnology Information. PubChem. Allylamine, $\mathrm{CID}=7853$." .

[45] H. Yasuda, Plasma Polymerization. Academic Press Inc, 1985.

[46] A. Alba-Perez, "Media coverage of our bone regeneration tech." [Online]. Available: https://glasgow.thecemi.org/2018/04/11/media-coverage-on-ourbone-regeneration-tech/.

[47] M. J. Dalby et al., "The control of human mesenchymal cell differentiation using nanoscale symmetry and disorder," Nat. Mater., vol. 6, no. 12, pp. 9971003, 2007.

[48] A. Wennerberg and T. Albrektsson, "Effects of titanium surface topography on 
bone integration: a systematic review," Clin. Oral Implants Res., vol. 20, no. s4, pp. 172-184, Sep. 2009.

[49] W. Orapiriyakul, P. S. Young, L. Damiati, and P. M. Tsimbouri, "Antibacterial surface modification of titanium implants in orthopaedics," J. Tissue Eng., vol. 9, p. 204173141878983 , Jan. 2018.

[50] P. M. Tsimbouri et al., "Osteogenic and bactericidal surfaces from hydrothermal titania nanowires on titanium substrates," Sci. Rep., vol. 6, no. 1, p. 36857 , Dec. 2016.

[51] P. Young, P. Tsimbouri, N. Gadegaard, R. Meek, and M. Dalby, "Osteoclastogenesis/osteoblastogenesis using human bone marrow-derived cocultures on nanotopographical polymer surfaces," Nanomedicine, vol. 10, no. 6, pp. 949-957, Mar. 2015.

[52] Y. Arima and H. Iwata, "Effect of wettability and surface functional groups on protein adsorption and cell adhesion using well-defined mixed self-assembled monolayers," Biomaterials, vol. 28, no. 20, pp. 3074-3082, 2007.

[53] C. Kleinhans et al., "Ammonia plasma treatment of polystyrene surfaces enhances proliferation of primary human mesenchymal stem cells and human endothelial cells," Biotechnol. J., vol. 8, no. 3, pp. 327-337, Mar. 2013.

[54] I. Retzko, J. F. Friedrich, A. Lippitz, and W. E. S. Unger, "Chemical analysis of plasma-polymerized films: The application of X-ray photoelectron spectroscopy (XPS), X-ray absorption spectroscopy (NEXAFS) and fourier transform infrared spectroscopy (FTIR)," J. Electron Spectros. Relat. Phenomena, vol. 121, no. 1, pp. 111-129, 2001.

[55] N. Vandencasteele and F. Reniers, "Plasma-modified polymer surfaces: Characterization using XPS,” J. Electron Spectros. Relat. Phenomena, vol. 178-179, pp. 394-408, 2010.

[56] H. F. Pereira, I. F. Cengiz, F. S. Silva, R. L. Reis, and J. M. Oliveira, "Scaffolds and coatings for bone regeneration," J. Mater. Sci. Mater. Med., vol. 31, no. 3, p. 27, 2020.

[57] H. D. Binks, "Spray Gun - US1650686A," 1927. 


\section{Appendices}

\subsection{Scientific publications by the candidate}

I have co-authored two scientific papers that present part of the results based on the technology for plasma polymerisation of PEA developed in this thesis. I am co-first author in both publications. These are referenced and briefly summarised here:

Nanoscale Coatings for Ultralow Dose BMP-2-Driven Regeneration of CriticalSized Bone Defects. Zhe A Cheng, Andres Alba-Perez, Cristina Gonzalez-Garcia, Hannah Donnelly, Virginia Llopis-Hernandez, Vineetha Jayawarna, Peter Childs, David W Shields, Marco Cantini, Laura Ruiz-Cantu, Andrew Reid, James FC Windmill, Elena S Addison, Sandra Corr, William G Marshall, Matthew J Dalby, Manuel Salmeron-Sanchez. Advanced Science, 2019, Volume 6, Issue 2, 1800361. https://doi.org/10.1002/advs.201800361

This paper reports first results from our plasma-polymerized PEA technology, showing high bioactivity from ultralow-dose growth factor delivery. We used our plasma reactor to deposit coatings on substrates to enhance the effect of BMP-2 in bone regeneration, with good results in vitro and in vivo, that remarkably included a veterinary patient.

In this first paper we did a basic physicochemical characterisation of the plasmapolymerized coatings. Only one of the deposition conditions was characterised, i.e. plasma incident power of $50 \mathrm{~W}$ and 30 minutes of depositions, as this was the one identified as optimal for most substrates and the one used for the biological experiments in the paper. A first AFM phase image of FN adsorbed on plasmapolymerized PEA was showed here, showing the compact and dense networks of the protein characteristic on this surface. We also characterised surface density of FN adsorbed at different concentrations, exposure of integrin-binding and GF-binding domains on adsorbed FN, adsorption of BMP-2 on FN-coated surfaces, and cumulative BMP-2 release from surfaces coated with plasma-polymerized PEA.

In vitro, we investigated whether BMP-2 presentation from plasma-polymerized PEA surfaces drives cell adhesion, enhances synergistic integrin/GF signalling, and is osteoinductive at low GF concentration. We cultured human mesenchymal stem cells (hMSCs) and characterised initial cell attachment, colocalization of vinculin (which stains focal adhesions) with BMP receptor 1A, phosphorylation of small mothers against decapentaplegics (SMAD) and phosphorylation of focal adhesion kinase (FAK). From our findings, we propose that enhanced synergistic adhesion and BMP2 signalling on $\mathrm{pPEA}+\mathrm{FN}+\mathrm{BMP}-2$ occurs as a consequence of the simultaneous occupancy of integrins and BMP-2 receptors. We next investigated the potential of this synergistic signalling cascade, driven by PEA-induced assembly of FN, for 
osteogenesis. Alkaline phosphatase, osteopontin and osteocalcin expression and Von Kossa staining was measured in hMSCs cultures.

In vivo, we showed healing of a critical-sized bone injury in a mice model and efficacy demonstrated in a veterinary patient - first in a veterinary trial still underway - a Münsterländer dog with a nonhealing humerus fracture, establishing a pathway to the clinical translation of this technology for ultralow-dose growth factor delivery.

Plasma polymerised nanoscale coatings of controlled thickness for efficient solidphase presentation of growth factors. Andres Alba, Vineetha Jayawarna, Peter G. Childs, Matthew J. Dalby, Manuel Salmeron-Sanchez. Materials Science and Engineering: C In Press, Journal Pre-proof. Available online 13 April 2020, 110966. https://doi.org/10.1016/j.msec.2020.110966

This second paper on the plasma polymerisation technology reports systematically process conditions and their impact on the plasma polymerised PEA coatings. We characterise the effect of plasma power and deposition time on thickness, wettability and chemical composition of the coatings. We demonstrate that functional substrate roughness can be maintained after deposition of the polymer coatings. Importantly, we show that coatings deposited at different conditions all maintain a similar or better bioactivity than spin coated PEA references.

We show in detail that in PEA plasma polymerised coatings fibronectin assembles into nanonetworks with high availability of integrin and GF binding regions that sequester bone morphogenetic protein-2 (BMP-2). We also report similar mesenchymal stem cell adhesion behaviour, as characterised by focal adhesions, and differentiation potential on BMP-2 coated surfaces, regardless of plasma deposition conditions.

\subsection{Plasma reactor operation protocol}

Our standard plasma polymerization protocol starts with a 5 minutes stage of air plasma at around 0.1 mbar and 100W (50W for PCL substrates) of continuous power to clean and activate surfaces. The air inlet valve is opened and adjusted to the required pressure to spark a plasma. A second stage of plasma polymerization is run with the monomer vial valve used as a throttle to control the chamber pressure while the plasma is started and maintained. The protocol is structured in seven distinctive steps:

Step 0. Review of experiment checklist. Preparation of samples for coating in reactor. Preparation and loading of monomer vial. Loading of cold trap with liquid N2.

Step 1. Lab air extraction on. Pump down with intake valves closed for about 3 to 5 minutes. Pressure reading at this point is around 0.01 mbar or lower if all rings and valves are working properly. This step is used to check for any leaks or problems with the vacuum before the continuation of the experiment. 
Step 2. Air intake valves are opened slowly and adjusted to a chamber pressure of around 0.1 mbar.

Step 3. The RF source is powered on at $100 \mathrm{~W}$ (50W for PCL) and the plasma glow discharge is visible. Air plasma is run for 5 minutes, at a controlled pressure of around 0.1 to 0.15 mbar with air intake valves used to maintain the chamber pressure within the established range.

Step 4. The RF source is shut off after 5 minutes of plasma. Air intake valves are closed and the system is pumped down for 1 to 2 minutes until pressure is again close to or below 0.01 mbar. This step is used to clean the system of any residues from the air plasma stage.

Step 5. The monomer inlet valve and monomer vial stopper are opened. The monomer vial stopper is used to control and adjust the evaporation and flow of monomer vapour into the chamber, to a chamber pressure of around 0.1 mbar. Usually 30 to 60 seconds of homogenization are allowed at this point to ensure a uniform distribution of the reactive vapour within the vacuum chamber.

Step 6. The RF source is powered on at the power defined for the experiment and the plasma glow discharge is visible, white colour for monomer. The monomer plasma is then left to run for the desired process time at a controlled pressure range that depends on the applied power, with intake valve and vial stopper used to maintain the chamber pressure within the established range.

Step 7. The RF power is turned off, the monomer vial valve closed and at least one pump/vent cycle is performed to extract monomer residues. The cold-trap is disassembled and cleaned. The monomer vial is stored in a designated fridge.

\subsection{Overview of coating protocols for scaffolds}

Together with several other researchers in the team we set preparation and coating protocols for scaffolds. Scaffolds of different types including commercial bone chips have been used by other researchers in the lab in their lines of work. Commercial allogeneic bone chips have been selected for a procedure of implant for recovery of long bone non-union fractures in a veterinary trial.

To standardize methods in the handling and preparation of these materials for in vivo experiments we prepared written protocols that detail from scaffold preparation to protein coating before use in surgery.

In Figure 58 below we summarize visually the steps followed for the preparation of scaffolds to be coated with plasma polymerized PEA and with protein for in vitro or in vivo testing. 


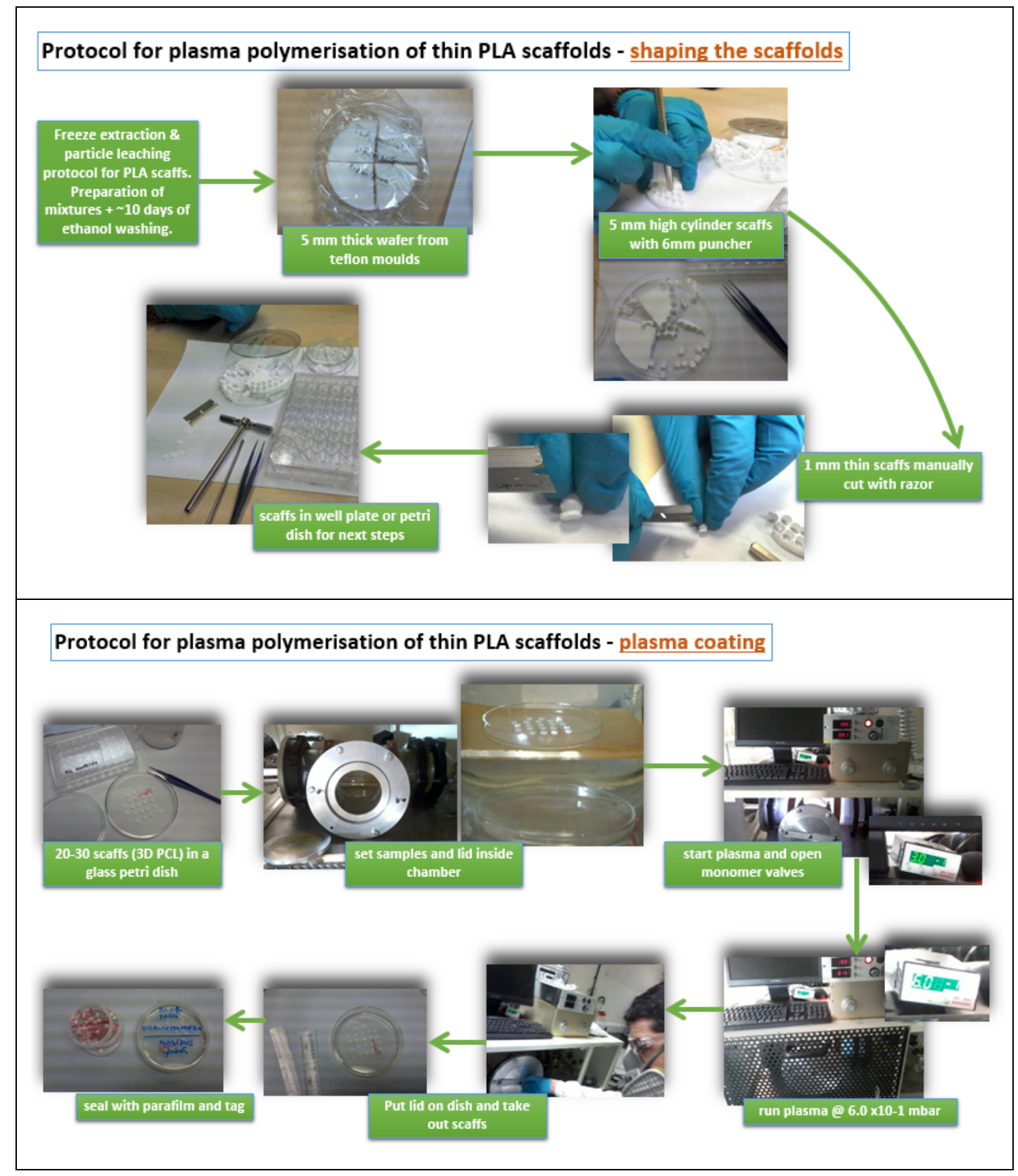




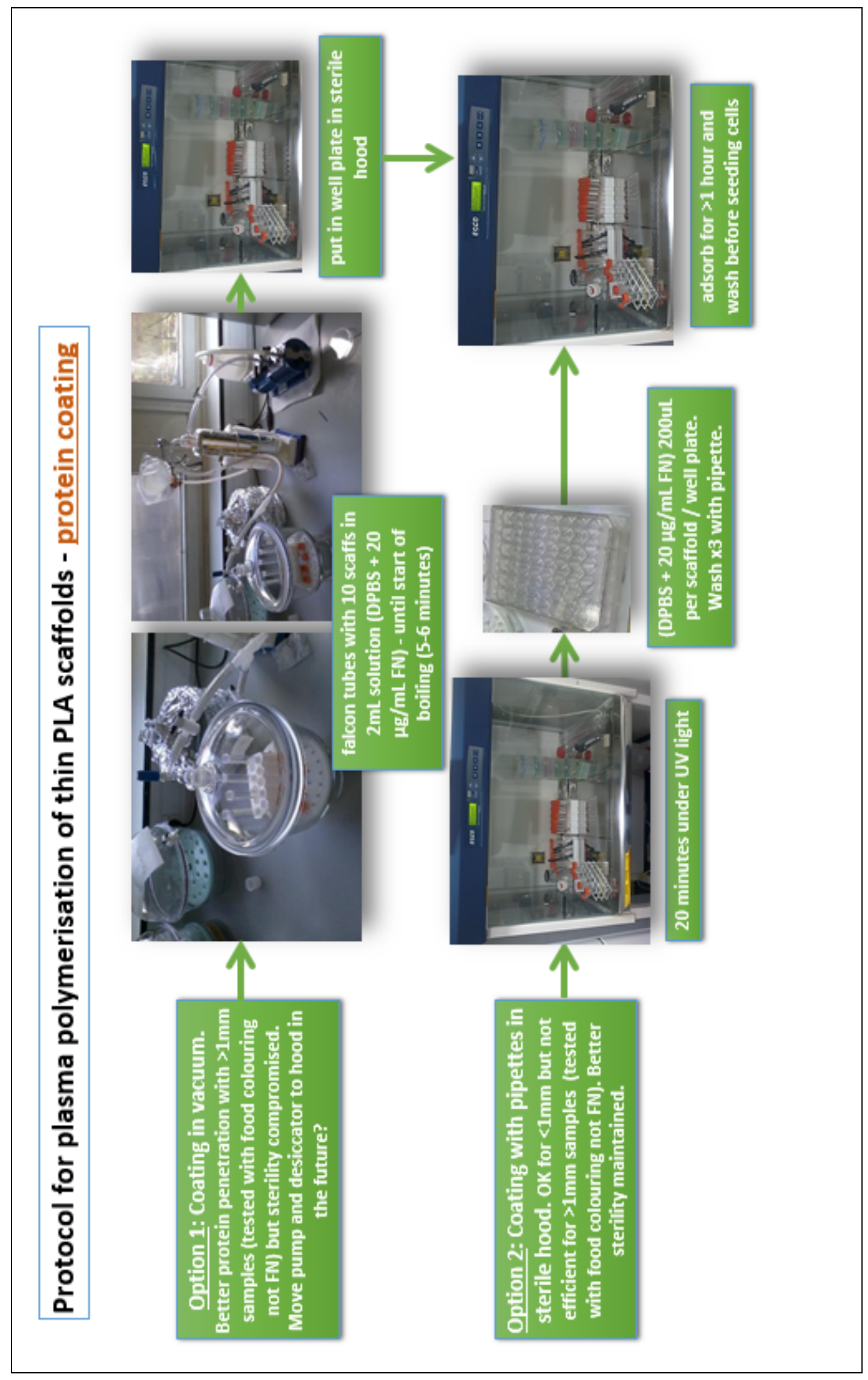




\subsection{List of figures}

Figure 1. Schematic diagram of cell - ECM interactions and material-driven FN fibrillogenesis.

Figure 2. Views of the three plasma polymerization reactor designs assembled in this work.

Figure 3. Schematic diagram and pictures of T-shape reactor plasma polymerisation installation.

Figure 4. Range of operating pressures for plasma at $100 \mathrm{~W}$.

Figure 5. Pictures of first cylindrical chamber plasma reactor prototype.

Figure 6. Plasma reactor prototype with a low volume cylindrical chamber.

Figure 7. Schematic diagram of plasma reactor prototype with cylindrical chamber.

Figure 8 . Measurement of coating thickness by a scratch test.

Figure 9. Plasma polymerized coatings. Thickness measurements.

Figure 10. Plasma polymerized coatings. Thickness measurements. Short deposition times.

Figure 11. Plasma deposition thickness in different areas of the reactor.

Figure 12. AFM scans of plasma polymerised surfaces. Control surfaces.

Figure 13. AFM scans of plasma polymerised surfaces, effect of duration of plasma. $50 \mathrm{~W}$ of incident $\mathrm{Rf}$ power.

Figure 14. AFM scans of plasma polymerised surfaces, effect of duration of plasma. 100W of incident Rf power.

Figure 15. AFM scans summary.

Figure 16. AFM scans plasma 100W, 30 minutes.

Figure 17. AFM scans plasma 50W, 30 minutes.

Figure 18. Flaky surfaces after plasma deposition.

Figure 19. AFM scans of spin coated PLLA surfaces with controlled roughness

Figure 20. AFM phase scans of spin coated PLLA surfaces with controlled roughness.

Figure 21. Roughness on coated rough samples.

Figure 22. Coating of hyper-rough surfaces, pits.

Figure 23. Coating of hyper-rough surfaces, nanowires.

Figure 24. Water contact angle measurements on plasma polymerised coatings. Compared to controls and by energy applied.

Figure 25. Water contact angle measurements on plasma polymerised coatings. Effect of storage and immersion in water.

Figure 26. Chemical composition of PEA surfaces taken by XPS analysis, by plasma deposition energy.

Figure 27. XPS analysis of PEA surfaces, variation between samples.

Figure 28. XPS analysis of PEA surfaces on PLA substrate. Amorphous PLA

Figure 29. XPS analysis of PEA surfaces on PLA substrate. Crystalline PLA.

Figure 30. AFM scans of FN coated surfaces. Controls.

Figure 31. AFM scans of FN coated surfaces. Plasma Rf incident power at 50W.

Figure 32. AFM scans of FN coated surfaces. Selected AFM scans at higher magnification 
Figure 33. AFM scans of FN coated surfaces. Plasma Rf incident power at 100W, 30 minutes.

Figure 34. AFM scans of FN coated surfaces. Plasma Rf incident power at 100W, 15 minutes.

Figure 35. AFM scans of FN coated surfaces. Plasma Rf incident power at 25W, 15 minutes.

Figure 36. Fluorescence microscopy images of protein coated surfaces.

Figure 37. Characterisation of PEA coatings before and after FN adsorption.

Figure 38. Conformation of FN at low concentration on plasma polymerised surfaces. $\mathrm{FN}$ at $1 \mu \mathrm{g} / \mathrm{ml}$.

Figure 39. Conformation of FN at low concentration on plasma polymerised surfaces. $\mathrm{FN}$ at $500 \mathrm{ng} / \mathrm{ml}$.

Figure 40. BMP-2 adsorption on plasma polymerized PEA.

Figure 41. Coating of scaffolds. Handling of samples.

Figure 42. Coating of scaffolds. Tests with PLS scaffolds and coating of bone chips for dog surgery.

Figure 43. Schematics of the spraying set-ups.

Figure 44. Airgun coatings. Optical microscopy pictures of sprayed coatings on glass surface.

Figure 45. Airgun coatings. Sprayed PEA surfaces. Roughness.

Figure 46. Airgun coatings. Sprayed PEA surfaces. Coating distribution and thickness. PEA at $1 \%$.

Figure 47. Airgun coatings. Sprayed PEA surfaces. Coating distribution and thickness. PEA at $8 \%$.

Figure 48. Airgun coatings. PLLA scaffolds coated with sprayed PEA

Figure 49. Airgun coatings. PLLA scaffolds coated with sprayed PEA, sudan blue.

Figure 50. Airgun coatings. PCL scaffolds coated with sprayed PEA, with sudan blue colouring.

Figure 51. Airgun coatings. AFM scans of FN adsorbed on PEA sprayed on glass coverslips

Figure 52. Airgun coatings. AFM scans of FN adsorbed on PEA sprayed on spin coated PLLA.

Figure 53. Airbrush coatings. Optical microscopy pictures of sprayed coatings on glass surfaces.

Figure 54. Airbrush coatings. Optical microscopy pictures of sprayed coatings on glass surfaces. Dilution of spraying solution in ethanol.

Figure 55. Airbrush coatings. Thickness measured with AFM scratch test.

Figure 56. Optical images of hydrolytic degradation of surfaces sprayed with PEA

Figure 57. Degradation of coated PCL scaffolds.

Figure 58. Overview of coating protocols for scaffolds. 
8.5 Schematic diagram of cell - ECM interactions and material-driven FN fibrillogenesis

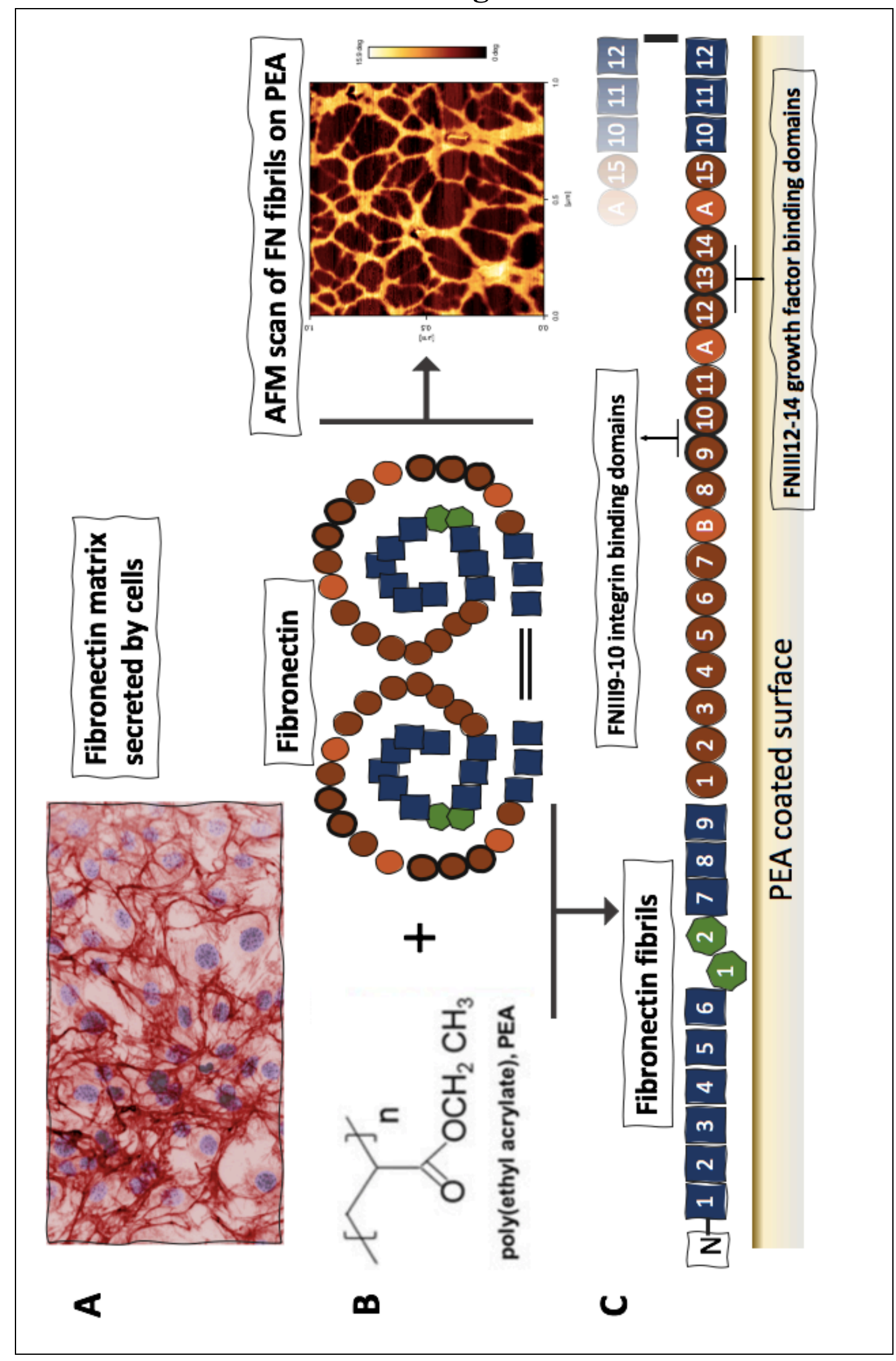






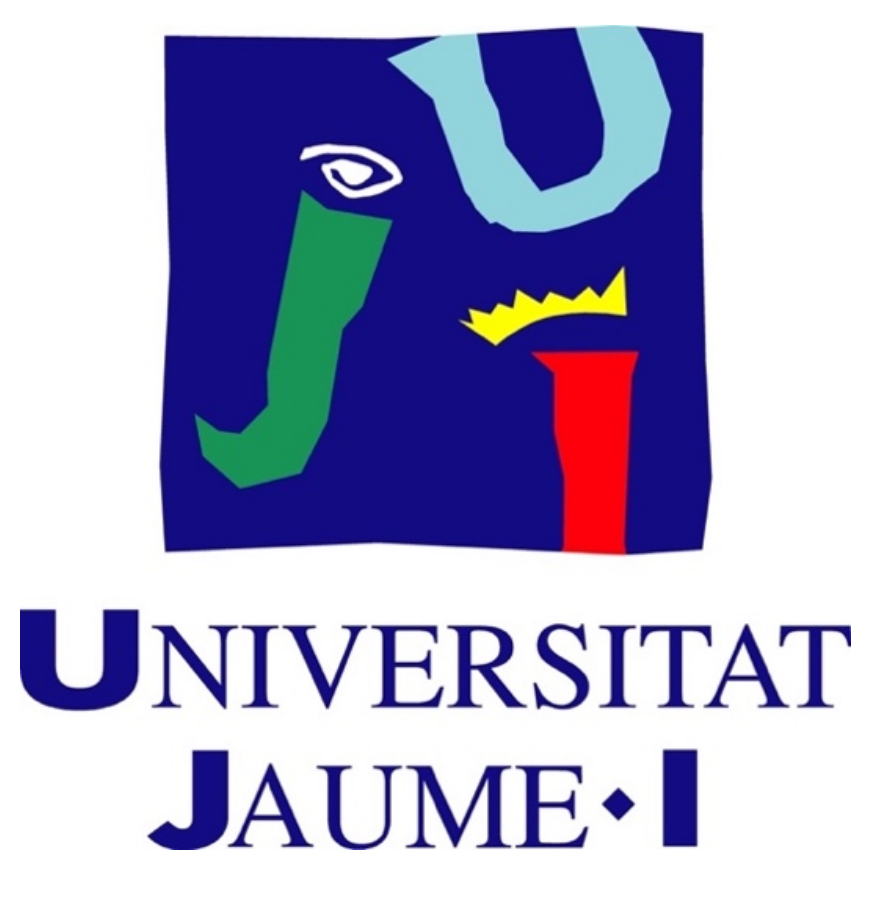

Doctoral Thesis

Coatings of Controlled Thickness for Efficient Solid-Phase Presentation of Growth Factors

\author{
Andrés Alba Pérez
}

October 2020

Director: Prof. Manuel Salmerón Sánchez

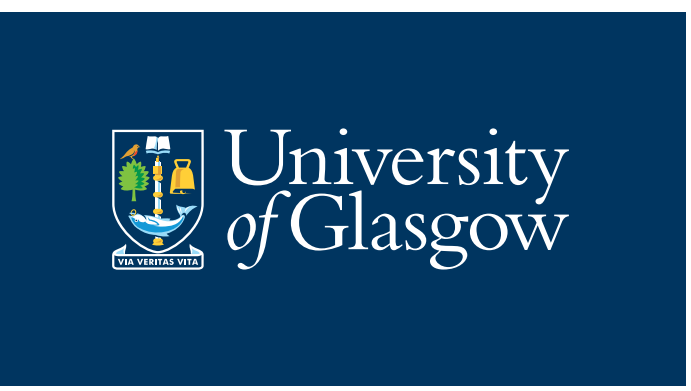


